\title{
Interaction Between Aerothermally Compliant Structures and Boundary-Layer Transition in Hypersonic Flow
}

\author{
Dissertation \\ Presented in Partial Fulfillment of the Requirements \\ for the Degree Doctor of Philosophy in the Graduate School \\ of The Ohio State University \\ By \\ Zachary Bryce Riley, M.S. \\ Graduate Program in Aeronautical and Astronautical Engineering
}

The Ohio State University 2016

Dissertation Committee:

Jack J. McNamara, Advisor

Jeffrey Bons

Datta Gaitonde

Sandip Mazumder

Benjamin Smarslok

S. Michael Spottswood 
Copyright by

Zachary Bryce Riley

2016 


\begin{abstract}
The use of thin-gauge, light-weight structures in combination with the severe aerothermodynamic loading makes reusable hypersonic cruise vehicles prone to fluidthermal-structural interactions. These interactions result in surface perturbations in the form of temperature changes and deformations that alter the stability and eventual transition of the boundary layer. The state of the boundary layer has a significant effect on the aerothermodynamic loads acting on a hypersonic vehicle. The inherent relationship between boundary-layer stability, aerothermodynamic loading, and surface conditions make the interaction between the structural response and boundary-layer transition an important area of study in high-speed flows.
\end{abstract}

The goal of this dissertation is to examine the interaction between boundary layer transition and the response of aerothermally compliant structures. This is carried out by first examining the uncoupled problems of: (1) structural deformation and temperature changes altering boundary-layer stability and (2) the boundary layer state affecting structural response. For the former, the stability of bound- 
ary layers developing over geometries that typify the response of surface panels subject to combined aerodynamic and thermal loading is numerically assessed using linear stability theory and the linear parabolized stability equations. $\mathrm{Nu}-$ merous parameters are examined including: deformation direction, deformation location, multiple deformations in series, structural boundary condition, surface temperature, the combined effect of Mach number and altitude, and deformation mode shape. The deformation-induced pressure gradient alters the boundarylayer thickness, which changes the frequency of the most-unstable disturbance. In regions of small boundary-layer growth, the disturbance frequency modulation resulting from a single or multiple panels deformed into the flowfield is found to improve boundary-layer stability and potentially delay transition. For the latter, transitional boundary-layer aerothermodynamic load models are developed and incorporated into a fundamental aerothermoelastic code to examine the impact of transition onset location, transition length and transitional overshoot in heat flux and fluctuating pressure on the response of panels. Results indicate that transitional fluid loading can produce larger thermal gradients, greater peak temperatures, earlier flutter onset, and increased strain energy accumulation as compared to a panel under turbulent loading. Sudden transition, with overshoot in heat flux and fluctuating pressure, occurring near the leading edge of the panel provides the most conservative estimate for determining the life of the structure.

Finally, the coupled interaction between boundary-layer transition and structural response is examined by enhancing the aerothermoelastic solver to allow for 
time-varying transition prediction as a function of the panel deformation and surface temperature. A kriging surrogate is developed to reduce the online computational expense associated with transition prediction within an aerothermoelastic simulation. For the configurations examined in this study, panel deformation has a more dominant effect on boundary-layer stability than surface temperature. Allowing for movement of the transition onset location results in characteristically different panel deformations due to spatial variation in the thermal bending moment. The response of the clamped panel is more sensitive to the transition onset location than the simply-supported panel. 


\section{Dedication}

The dedication is split five ways for the years it took to complete this work. To my loving wife Michelle, for your support and motivation. To my parents Cathy and Rob, without whom none of this would have been possible. To my grandfather Raymond Riley, for the inspiration and the stories. To my dog Cassie, for keeping my lap warm as I write this. 


\section{Acknowledgments}

I would like to thank my advisor, Professor Jack McNamara, for his guidance and support throughout this work. His early confidence in me has been vital to my academic and professional growth. I would also like to thank Professors Datta Gaitonde, Sandip Mazumder, and Jeffrey Bons for serving on my candidacy and dissertation committees, and Drs. S. Michael Spottswood and Benjamin Smarslok for serving on my dissertation committee.

I am immensely grateful to the people and organizations that financially supported this work. This research was conducted with government support under and awarded by the Air Force Office of Scientific Research (AFOSR) through Grant FA9550-11-1-0036, with Dr. John Schmisseur as Program Manager; by the DoD through a National Defense Science and Engineering Graduate Fellowship (32 CFR 168a); and the AFRL-University Collaborative Center in Structural Sciences (AFRL/RQ Cooperative Agreement FA8650-13-2-2347) with Dr. Benjamin Smarslok as program manager. Additional support was provided by the AFOSR sponsored U.S. AFRL Summer Faculty Fellowship Program and by the AFRL Aero- 
space Systems Directorate Short-Term Research and Development Program for my time spent as a summer researcher at the AFRL. I would also like to thank the Ohio Supercomputer Center for providing computational resources.

I am deeply indebted to those whose technical insight has improved the quality of my research. I would like to thank Dr. Heath Johnson of VirtusAero LLC for supplying me with and providing guidance in the usage of STABL. Thanks are also due to Dr. Katya Casper of Sandia National Laboratories for providing insight and experimental pressure measurements for transitional boundary layers. Additionally, I greatly appreciate the guidance and mentorship of Drs. Ravi Chona, S. Michael Spottswood, Tom Eason, and Ben Smarslok of the AFRL/RQHF Structural Sciences Center. Finally, I must acknowledge Dr. Adam Culler, Dr. Brent Miller, Dr. Benjamin Grier, Rohit Deshmukh, and Kirk Brouwer who are all former or current members of Professor McNamara's research group. I would like to thank Dr. Culler for supplying numerical data used in the NASP ramp panel study, Dr. Miller for the development and maintenance of an aerothermoelastic panel response code, Dr. Grier for his assistance with mesh generation, Rohit Deshmukh for providing me with the turbulent boundary layer fluctuating pressure model, and Kirk Brouwer for guidance on the generation of kriging surrogates.

I would like to thank my fellow labmates Rohit Deshmukh, Brent Miller, Steve Nogar, Andrew Crowell, Kelsey Shaler, Ben Grier, Kirk Brouwer, Emily Dreyer, Zach Witeof, Joe Connolly, and everyone else for making this an enjoyable experience. I would like to thank my wife Michelle for her constant encouragement and 
support. I am grateful to my siblings Cory, Jenn, Rob, Amanda, and Hannah for always being there for me. I would also like to thank my parents, Robert and Cathy Thompson, for their love and support. Finally, thank you to all of my friends and family that believed in me and supported me throughout this process. 


\section{Vita}

2011

B.S. Aeronautical and Astronautical Engineering, The Ohio State University

2014

M.S. Aeronautical and Astronautical Engineering, The Ohio State University

2011-2012, 2016 ..... . Graduate Research Assistant, The Ohio State University

2012-2016 ........... NDSEG Fellow, The Ohio State University

\section{Publications}

\section{Journal Publications}

Riley, Z. B., Deshmukh, R., Miller, B. A., McNamara, J. J., and Casper, K. M., "Characterization of Structural Response to Hypersonic Boundary-Layer Transition," AIAA Journal, Vol. 54, No. 8, 2016, pg. 2418-2431. 
Riley, Z. B., McNamara, J. J., and Johnson, H. B., "Assessing Hypersonic Boundary Layer Stability in the Presence of Structural Deformation," AIAA Journal, Vol. 52, No. 11, 2014, pg. 2547-2558.

\section{Conference Publications}

Riley, Z. B., Grier, B., and McNamara, J. J., Numerical Assessment of Boundary Layer Stabilization due to Axisymmetric Roughness Strips in Hypersonic Flow, 46th AIAA Fluid Dynamics Conference, June 13-17, Washington, D.C., ITAR Paper, 2016.

Riley, Z. and McNamara, J., Interaction between Aerothermally Compliant Structures and Boundary Layer Transition, IMAC-XXXIV, January 25-28, Orlando, FL, 2016.

Riley, Z. B., and McNamara, J. J., Interaction Between Aerothermally Compliant Structures and Boundary Layer Transition in Hypersonic Flow, 15th Dynamics Specialists Conference, January 4-8, San Diego, CA, AIAA Paper 2016-1087, 2016.

Riley, Z., Deshmukh, R., Miller, B. A., and McNamara, J. J., Characterization of Structural Response to Hypersonic Boundary Layer Transition, 56th AIAA/ASCE/ AHS/ASC Structures, Structural Dynamics, and Materials Conference, January 5-9, 
Kissimmee, FL, AIAA 2015-0688, 2015.

McNamara, J. J., Crowell, A. R., Miller, B. A., Riley, Z. B., Deshmukh, R., and Culler, A. J., Structural Response Prediction of Surface Panels in Hypersonic Flow, International Forum on Aeroelasticity and Structural Dynamics, Bristol, UK, IFASD2013-29C, June 2013.

Riley, Z., and McNamara, J., Assessing Hypersonic Boundary Layer Stability in the Presence of Panel Scale Protuberances, 54th AIAA/ASME/ASCE/AHS/ASC Structures, Structural Dynamics, and Materials Conference, April 8-11, Boston, MA, AIAA 2013-1745, 2013.

Riley, Z., McNamara, J. and Johnson, H., Hypersonic Boundary Layer Stability in the Presence of Thermo-Mechanical Surface Compliance, 53rd AIAA/ASME/ ASCE/AHS/ASC Structures, Structural Dynamics, and Materials Conference, April 2326, Honolulu, HI, AIAA 2012-1549, 2012.

\section{Fields of Study}

Major Field: Aeronautical and Astronautical Engineering 


\section{Table of Contents}

Abstract ..................... ii

Dedication .......................

Acknowledgments .................... vi

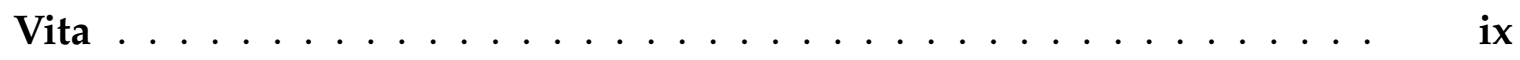

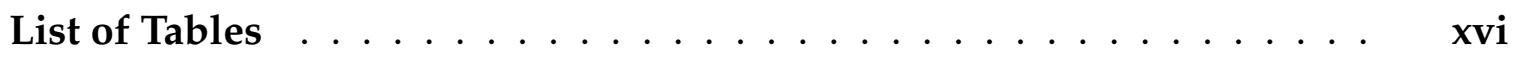

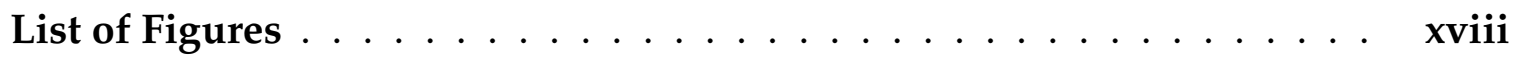

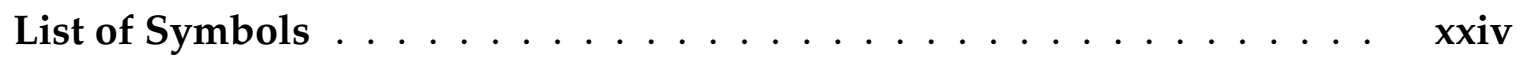

Chapter

1 Introduction and Objectives .................. 1

1.1 Introduction ................... 1

1.2 Literature Review . . . . . . . . . . . . . . . . . 9

1.2.1 Boundary-Layer Stability and Transition . . . . . . . . . . 10

1.2.1.1 History and Overview . . . . . . . . . 10

1.2.1.2 Boundary-Layer Stability Analysis . . . . . . . 12

1.2.1.3 Transition Prediction . . . . . . . . . . 21

1.2.2 Physical Parameters that affect Hypersonic Boundary-Layer

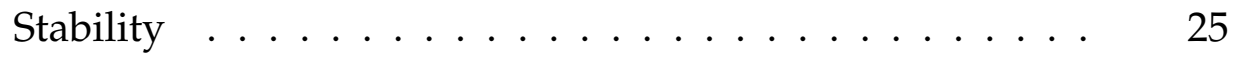


1.2.2.1 Surface Temperature . . . . . . . . . . . . . 29

1.2.2.2 Surface Perturbations . . . . . . . . . . . . 34

1.2.3 Aerothermoelastic Response of Structures in Hypersonic Flow 36 1.2.3.1 Experiments . . . . . . . . . . . . . 37

1.2.3.2 Computational Analysis . . . . . . . . . . . . 39

1.2.4 Potential Impact of Transitional Boundary Layer on Structural Response . . . . . . . . . . . . . . . . . . . 44

1.3 Objectives of this Dissertation . . . . . . . . . . . . 46

1.4 Key Novel Contributions of this Dissertation . . . . . . . . . 48

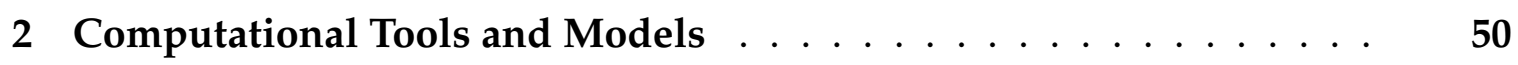

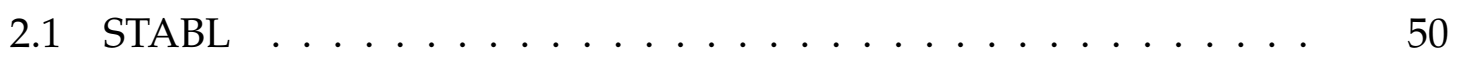

2.1.1 DPLR Mean Flow Solver . . . . . . . . . . . . . . . . 51

2.1.2 PSE-Chem: Boundary-Layer Stability Analysis . . . . . . 52

2.1.3 Transition Prediction . . . . . . . . . . . . . . . . 57

2.1.4 STABL Results . . . . . . . . . . . . . . . . . . 58

2.2 Surrogate Modeling . . . . . . . . . . . . . . . . . 61

2.3 Aerothermoelastic Model . . . . . . . . . . . . . . . . . . 63

2.3.1 Enhancements to the Aerothermoelastic Model . . . . . . 67

2.3.2 Aerothermodynamic Loads . . . . . . . . . . . . . 70

2.3.2.1 Piston Theory . . . . . . . . . . . . . . 70

2.3.2.2 Fluctuating Pressure Model . . . . . . . . . . . 71

2.3.2.3 Eckert's Reference Enthalpy _. . . . . . . . . 76

2.3.3 Transitional Aerothermodynamic Loads . . . . . . . . . 77

2.3.3.1 Intermittency . . . . . . . . . . . . 78

2.3.3.2 Heat Flux . . . . . . . . . . . . . . . . 79

2.3.3.3 Fluctuating Pressure . . . . . . . . . . . . 81

2.3.3.4 Experimental Comparisons . . . . . . . . . . 84

2.3.4 Thermal Model . . . . . . . . . . . . . . . . . . . . . . 89 
2.3.5 Structural Model . . . . . . . . . . . . . . . . . . . 9 90

2.4 Generic Panel Configuration . . . . . . . . . . . . . . . . 94

3 Impact of Surface Conditions on Hypersonic Boundary-Layer Stability 96

3.1 Panel Geometries . . . . . . . . . . . . . . . . . . . . . 97

3.1.1 Generic Metallic Panel . . . . . . . . . . . . . . . . 97

3.1.2 NASP Ramp Panel . . . . . . . . . . . . . . . . . . . 97

3.1.3 Spherical Domes . . . . . . . . . . . . . . . . . 102

3.1.4 Nondimensional Panel Comparison . . . . . . . . . . 105

3.2 Mean Flow Grid Convergence . . . . . . . . . . . . . . . 106

3.3 Results . . . . . . . . . . . . . . . . . . 107

3.3.1 Generic Metallic Panel . . . . . . . . . . . . . . . . 110

3.3.1.1 Panel Location . . . . . . . . . . . . . . . . 110

3.3.1.2 Multiple Panels in Series . . . . . . . . . . . 115

3.3.2 NASP Ramp Panel . . . . . . . . . . . . . . . . . . . . . 120

3.3.3 Spherical Domes . . . . . . . . . . . . . . . . 128

4 Impact of Boundary-Layer Transition on Structural Response . . . . 131

4.1 Problem Description . . . . . . . . . . . . . . . . . . . . 132

4.2 Panel Response for Laminar and Turbulent Flow . . . . . . . . 133

4.3 Transitional Overshoot Characterization . . . . . . . . . . . . 134

4.4 Effect of Transition Length on Panel Response . . . . . . . . . . 136

4.5 Effect of Transition Location on Panel Response . . . . . . . . . . 140

4.6 Effect of Transition on Flutter Onset . . . . . . . . . . . . 142

4.7 Effect of Temperature-Dependent Material Properties . . . . . . 148

4.8 Effect of Transition on Panel Life Considerations . . . . . . . . . 155

5 Interaction between Boundary-Layer

Transition and Structural Response . . . . . . . . . . . . . 157

5.1 Problem Description $\ldots \ldots \ldots \ldots \ldots \ldots$ 
5.2 Transition Onset Surrogate Model . . . . . . . . . . . . . . . . . . 159

5.2.1 Mean Flow and LPSE Grid Convergence . . . . . . . . . 160

5.2 .2 Surrogate Generation . . . . . . . . . . . . . . . . . 161

5.2 .3 Surrogate Verification . . . . . . . . . . . . . . . 173

5.2.4 Clamped Panel Surrogate Modifications . . . . . . . . . 176

5.3 Results . . . . . . . . . . . . . . . . . . . . . . . 179

5.3.1 Effect of Panel Response on Boundary-Layer Transition . . 180

5.3.1.1 Importance of Panel Deformation and Surface Temperature . . . . . . . . . . . 180

5.3.1.2 Physical Explanation for Movement of Transition with Panel Deformation . . . . . . . . . . . . . 184

5.3.2 Effect of Fluid Loading on Panel Response _. . . . . . . 190

5.3.3 Structural Sensitivity to the Transitional $N$ Factor _... . 203

6 Concluding Remarks . . . . . . . . . . . . . . . . . . 210

6.1 Principal Conclusions . . . . . . . . . . . . . . . . 210

6.2 Key Uncertain Parameters . . . . . . . . . . . . . . . . . 216

6.3 Recommendations for Future Work . . . . . . . . . . . . . 217

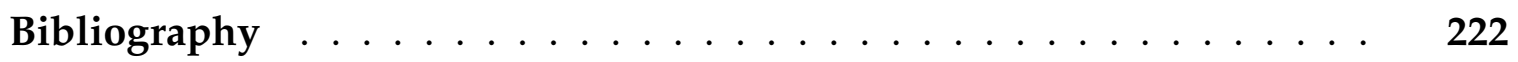




\section{List of Tables}

1.1 Basic state assumptions of linear stability methods (adapted from

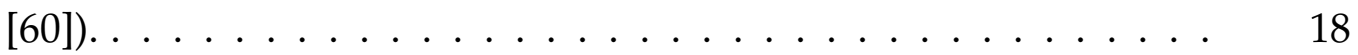

1.2 Perturbation assumptions of linear stability methods (adapted from

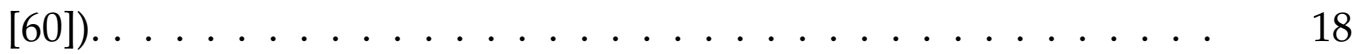

2.1 Numerical parameters of the aerothermoelastic solution. . . . . . 67

3.1 Geometry and freestream conditions for generic metallic panel study. 99

3.2 Geometry for NASP ramp panel ascent trajectory. . . . . . . . . 100

3.3 Flow conditions for NASP ramp panel ascent trajectory. . . . . . . 100

3.4 Spherical dome dimensions [93]. . . . . . . . . . . . . 102

3.5 Geometry and flow conditions for spherical domes. . . . . . . . . 105

3.6 Nondimensional deformation comparison. . . . . . . . . . 106

4.1 Freestream conditions. . . . . . . . . . . . . . . . 132

4.2 Panel geometry. . . . . . . . . . . . . . . . . . . 133

4.3 Temperature dependence of material properties of Aluminum (Al-

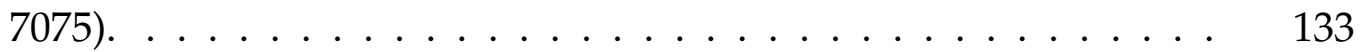

5.1 Freestream conditions. . . . . . . . . . . . . . . 159

5.2 Panel geometry. . . . . . . . . . . . . . . . . 159

5.3 Temperature dependence of material properties of Titanium (Ti6Al-2Sn-4Zr-2Mo). . . . . . . . . . . . . . . . 159 
5.4 Bounds for surrogate input parameters. . . . . . . . . . . . 164

5.5 Cross-validation approach for $n_{s p}=800$ surrogates. . . . . . 165

5.6 Computational cost for $5 \mathrm{~s}$ of aerothermoelastic panel response. . 176 


\section{List of Figures}

1.1 Conceptual hypersonic cruise vehicles. . . . . . . . . . . . . 4

1.2 Dependence of adiabatic wall temperature on the freestream Mach number. . . . . . . . . . . . . . . . . . . 6

1.3 Schematic of fluid-thermal-structural interaction. . . . . . . . . 8

1.4 Boundary-layer transition process (figure 5-28.a of [58]). . . . . . . 13

1.5 Agreement of $e^{10}$ predicted transitional Reynolds number with experimental data (figure 13 of [72]). . . . . . . . . . . . . 23

1.6 Effect of Mach number on hypersonic boundary-layer stability and

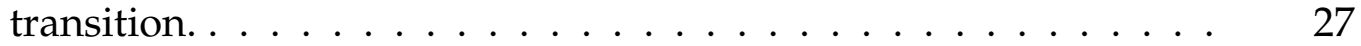

1.7 Movement of predicted transition location with wall cooling at $M_{\infty}=$ $5, T_{\infty}=300 \mathrm{~K}$ (adapted from figure 11.a of [67]). . . . . . . 31

2.1 Shadowgraph highlighting flow modification due to surface deformation in hypersonic flow. . . . . . . . . . . . . .

2.2 LST and LPSE results for Mach 5 flow past a $5^{\circ}$ half-angle smooth wedge in Mach 5 flow. . . . . . . . . . . . . . . . . 60

2.3 Kriging surrogate example. . . . . . . . . . . . . . . . 64

2.4 Aerothermoelastic model. . . . . . . . . . . . . . . . . . . 66

2.5 Power spectral density at 3/4-chord length (transitional overshoot $\left.x_{t}=0.3 \mathrm{~m}, x_{t e}=0.5 \mathrm{~m}\right) \ldots \ldots \ldots \ldots \ldots \ldots \ldots \ldots \ldots$

2.6 Enhanced aerothermoelastic model. . . . . . . . . . . . 70 
2.7 Turbulent boundary-layer fluctuating pressure model comparison with the experimental data of Beresh et al. [142]. RMS pressure normalized by dynamic pressure at boundary-layer edge. . . . . .

2.8 Transitional heat flux model. Transition beginning at $\bar{x}_{t}=0.3$ and ending at $\bar{x}_{t e}=0.5 \ldots \ldots \ldots \ldots \ldots \ldots \ldots$

2.9 Transitional fluctuating pressure load model. Transition beginning at $\bar{x}_{t}=0.3$ and ending at $\bar{x}_{t e}=0.5 \ldots \ldots \ldots \ldots$

2.10 Transitional heat flux for $x_{t}=0.35 \mathrm{~m}$ and $\Delta x_{t}=0.21 \mathrm{~m}$ (experi-

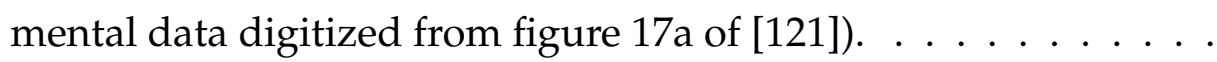

2.11 Transitional RMS pressure normalized by boundary-layer edge pressure for $x_{t}=0.20 \mathrm{~m}$ and $\Delta x_{t}=0.084 \mathrm{~m}$ (experimental RMS pressure values are totals between 0 and $1 \mathrm{MHz}$ from [29]). . . . . . .

2.12 Transitional PSDs for $x_{t}=0.20 \mathrm{~m}$ and $\Delta x_{t}=0.084 \mathrm{~m}$. Lines with markers denote experimental data digitized from figure 9c of [29].

2.13 Thermal Model. . . . . . . . . . . . . . . . . . . . . . . . . . . . 89

2.14 Structural Model. . . . . . . . . . . . . . . . . . . . . . . . . . 91

2.15 Compliant panel located on 2-D surface inclined to freestream. . . 95

3.1 Representative thermostructural responses extracted from [35] for a generic metallic panel. . . . . . . . . . . . . . . . . . 98

3.2 NASP-era ramp panel (figure 3 of [101]) . . . . . . . . . . . 100

3.3 Representative responses of NASP ramp panel. . . . . . . . . . . 101

3.4 Schematic of Glass and Hunt experimental geometry with dimensions in inches (figure 2 of [93]) . . . . . . . . . . . . . 103

3.5 Displacement profiles for spherical and clamped dome configura-

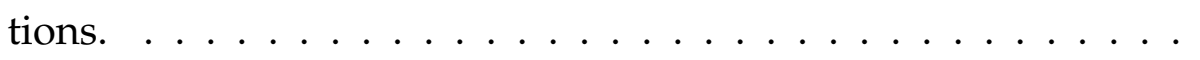

3.6 Wall-normal derivatives at peak deformation location (Glass and Hunt dome 6 [93]). The legend indicates the number of wall normal grid points and either $1^{s t}$ or $2^{n d}$ derivative. . . . . . . . 108 
3.7 LPSE resolution study for the maximum deformation case (Glass and Hunt dome 6 [93]). . . . . . . . . . . . . . . . . . . . . . 109

3.8 Stability diagrams for different panel locations. . . . . . . . . . . 112

3.9 Boundary-layer thickness for rigid wedge and panel response at 1 m. . . . . . . . . . . . . . . . . . . . . 113

3.10 Maximum $N$ factor comparison for different panel response locations. . . . . . . . . . . . . . . . . . . . . . 114

3.11 Configuration for three convex deformations in series. . . . . . . 116

3.12 Stability diagrams for multiple panels in series. . . . . . . . . . 117

$3.13 N$ factor comparison for multiple panels in series. . . . . . . . . 119

3.14 Maximum $N$ factors for rigid wedge during ascent. . . . . . . . 121

3.15 Boundary-layer thickness for cold and radiative equilibrium smooth wall during ascent trajectory. . . . . . . . . . . . . . . . 123

3.16 Stability diagrams for smooth wall geometry at $90 \mathrm{~s}$ into ascent trajectory $\left(M=5, R e=32.91 \times 10^{6}\right) . \ldots \ldots \ldots \ldots$

3.17 Maximum $N$ factors for panel response during ascent. . . . . . . 126

3.18 Impact of panel deformation pattern on boundary-layer growth and stability for cold wall. . . . . . . . . . . . . . . . 127

3.19 Maximum $N$ factor growth for domes 1 to $6 . \ldots \ldots \ldots$

4.1 Panel response for laminar and turbulent flows. Times in Fig. 4.1.a correspond to laminar and turbulent flutter onsets. . . . . . . . . 135

4.2 Average overshoot percentage for transitional cases with the same $\Delta x_{t}$ but different $x_{t} \ldots \ldots \ldots \ldots \ldots \ldots \ldots \ldots$

4.3 Variation in panel response due to transition length $\left(\bar{x}_{t}=0.1\right)$ : no overshoot "no" (gray lines and open markers), and overshoot "os" (black lines and closed markers). . . . . . . . . . . . . . . . . . 139

4.4 Panel response subject to turbulent and transitional $\left(\bar{x}_{t}=0.1, \bar{x}_{t e}=\right.$ 0.25 ) loading with overshoot at $23.0 \mathrm{~s} . \ldots \ldots \ldots \ldots$ 
4.5 Variation in panel response due to transition location $\left(\Delta \bar{x}_{t}=0.15\right)$ : no overshoot "no" (gray lines and open markers), and overshoot "os" (black lines and closed markers). . . . . . . . . . . . . . . . .

4.6 Time to flutter. Laminar flutter condition of $t_{F}=142.5 \mathrm{~s}$ is not

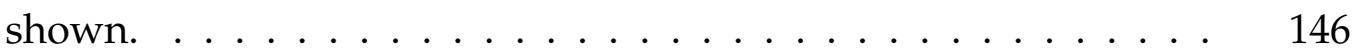

4.7 Variation in flutter time due to overshoot. . . . . . . . . . . . . 147

4.8 Chordwise average temperature at flutter. . . . . . . . . . . . . . 149

4.9 Maximum displacement before flutter. . . . . . . . . . . . . 150

4.10 Through-thickness average temperature profile at flutter onset. . 151

4.11 Temperature-dependent material properties at flutter onset. . . . 153

4.12 Effect of temperature dependence on flutter time: no overshoot "no" (open markers), and overshoot "os" (closed markers). . . . . 154

4.13 Strain energy accumulation during the first $20 \mathrm{~s}$ of response. . . . 156

5.1 Shadowgraph of flowfield $\left(a_{1} / h=4.204, a_{2} / h=-0.374, a_{3} / h=\right.$

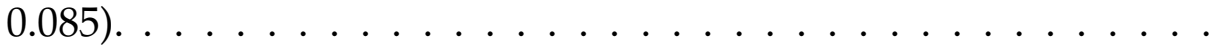

5.2 Wall normal derivatives at midpoint of panel $S=2.75 \mathrm{~m}\left(T_{w}=300\right.$ $\left.\mathrm{K}, a_{1-3} / h=4.204,-0.374,0.085\right) \ldots \ldots \ldots 162$

5.3 LPSE resolution study $\left(T_{w}=300 \mathrm{~K}, a_{1-3} / h=4.204,-0.374,0.085\right) . \quad 163$

5.4 Surrogate convergence with increasing sample points. . . . . . . . 167

5.5 Model reduction error. . . . . . . . . . . . . . . . . . . 169

5.6 Panel response at $10 \mathrm{~s}$ due to time-varying transition region $\left(\Delta x_{t}=\right.$ $0.30 \mathrm{~m}) \ldots \ldots \ldots \ldots 171$

5.7 Impact of wall temperature gradient on transition prediction. . . . 172

5.8 Intermittency-based blending of minimum and average temperature $N$ factor curves. . . . . . . . . . . . . . . . 173

5.9 Comparison of the aerothermoelastic panel responses obtained using STABL and the surrogate for transition prediction. . . . . . . . 
5.10 Error associated with approximating clamped panel deformation as simply-supported. . . . . . . . . . . . . . . . . . . 178

5.11 Maximum $N$ factor envelope for clamped and simply-supported deformations. . . . . . . . . . . . . . . . . 179

5.12 Movement of the transition onset location due to the panel response. 181

5.13 Variation in the transition onset location due to the panel response. 183

5.14 Effect of panel displacement on $N$ factor growth. . . . . . . . . 186

5.15 Effect of panel deformation on the flowfield. . . . . . . . . . 187

5.16 Effect of panel deformation on the second-mode disturbance frequency. Contour values greater than zero indicate unstable growth. 188

5.17 Effect of adverse pressure gradient on $N$ factor growth. . . . . . 189

5.18 Maximum panel temperature subject to fully turbulent and transitional (fixed and time-varying) loading. . . . . . . . . . . . . . 191

5.19 Average panel temperature subject to fully turbulent and transitional (fixed and time-varying) loading. . . . . . . . . . . . .

5.20 Center displacement of panel subject to fully turbulent and transitional (fixed and time-varying) loading. . . . . . . . . . . . .

5.21 Variation in simply-supported panel response due to fixed (dashed lines) and time-varying (solid lines) transitional loading. . . . . .

5.22 Variation in clamped panel response due to fixed (dashed lines) and time-varying (solid lines) transitional loading. . . . . . . . . .

5.23 Variation in simply-supported panel displacement at $10 \mathrm{~s}$ due to material temperature dependence. . . . . . . . . . . . . . . . 198

5.24 Importance of thermal moment on simply-supported panel response. 200

5.25 Impact of step function thermal moment on simply-supported panel displacement. . . . . . . . . . . . . . . . . . 202

5.26 Impact of step function thermal moment on panel displacement. No pressure loading. . . . . . . . . . . . . . . . . . . . 204 
5.27 Movement of the transition front for variation in transitional $N$ fac-

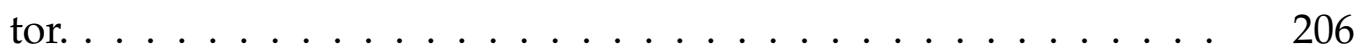

5.28 Center displacement with variation in transitional $N$ factor. . . . 208

5.29 Average panel temperature with variation in transitional $N$ factor. 209 


\section{List of Symbols}

$\begin{array}{ll}a & \begin{array}{l}\text { speed of sound } \\ a_{i}\end{array} \\ b & \text { amplitude of } i \text { th free vibration mode } \\ C C & \text { compressibility exponent } \\ C W & \text { clamped-clamped boundary condition } \\ c & \text { specific heat capacity } \\ c_{f} & \text { skin friction coefficient } \\ E & \text { modulus of elasticity } \\ F_{c} & \text { compressible flow transformation function } \\ H & \text { deformation height, enthalpy } \\ h & \text { panel thickness } \\ k & \text { thermal conductivity } \\ L & \text { deformation/panel length } \\ M & \text { Mach number } \\ M_{T} & \text { thermal bending moment } \\ N & \text { transition correlation factor } \\ N_{E R R O R} & \text { percent error in } N \text { factor } \\ N_{T} & \text { average thermal force } \\ n & \text { aerodynamic pressure }\end{array}$

xxiv 


$\begin{array}{ll}P r & \text { Prandtl number } \\ p & \text { unsteady aerodynamic pressure } \\ \tilde{p} & \text { RMS fluctuating pressure } \\ Q & \text { aerodynamic heat flux } \\ Q_{r a d} & \text { radiative heat flux } \\ q & \text { dynamic pressure } \\ R E & \text { radiative equilibrium temperature } \\ R I G & \text { rigid/smooth wall } \\ R e & \text { length Reynolds number } \\ R e_{\theta} & \text { momentum thickness Reynolds number } \\ r & \text { recovery factor } \\ S S & \text { simply-supported boundary condition } \\ S & \text { surface distance, relative to wedge geometry } \\ S_{E R R O R} & \text { percent error in the onset location } \\ T & \text { temperature } \\ T u & \text { turbulence intensity } \\ t & \text { time } \\ t_{F} & \text { panel flutter onset time } \\ U, u & \text { streamwise component of fluid velocity } \\ V, v & \text { wall-normal component of fluid velocity } \\ w & \text { transverse panel displacement } \\ x & \text { chordwise spatial coordinate, relative to panel } \\ \bar{x} & \text { spatial location nondimensionalized by panel length } \\ x_{t} & \text { transition onset location } \\ x_{t e} & \text { end of transition region } \\ z & \text { through-thickness spatial coordinate, relative to panel } \\ \alpha & \\ & \text { streamwise wavenumber, thermal expansion coefficient } \\ & \end{array}$




\begin{tabular}{|c|c|}
\hline$\beta$ & standard deviation \\
\hline$\gamma$ & intermittency, ratio of specific heats \\
\hline$\Delta x_{t}$ & transition length $\left(x_{t e}-x_{t}\right)$ \\
\hline$\delta$ & boundary-layer thickness \\
\hline$\delta_{1}$ & boundary-layer displacement thickness \\
\hline$\epsilon$ & emissivity \\
\hline$\Theta$ & overall phase angle \\
\hline$\lambda$ & viscosity/velocity power law exponent \\
\hline$\mu$ & dynamic viscosity \\
\hline$\nu$ & Poisson's ratio, kinematic viscosity \\
\hline$\rho$ & density \\
\hline$\sigma$ & spot propagation parameter \\
\hline$\tau$ & temporal phase angle \\
\hline$\phi$ & power spectral density \\
\hline$\psi$ & spatial phase angle \\
\hline$\omega$ & angular frequency \\
\hline \multicolumn{2}{|c|}{ Subscripts } \\
\hline$\infty$ & freestream property \\
\hline$A E$ & aeroelastic \\
\hline$A T$ & aerothermal \\
\hline $\operatorname{avg}$ & average \\
\hline$e$ & boundary-layer edge \\
\hline$I S O$ & isothermal \\
\hline init & initial \\
\hline$i$ & imaginary component \\
\hline $\operatorname{lam}$ & laminar \\
\hline $\max$ & maximum \\
\hline
\end{tabular}




$\begin{array}{ll}\min & \text { minimum } \\ \text { tran } & \text { transitional } \\ \text { turb } & \text { turbulent } \\ \mathrm{VO} & \text { virtual origin } \\ w & \text { wall }\end{array}$

Superscripts

Eckert's reference enthalpy condition 


\section{Chapter 1}

\section{Introduction and Objectives}

\subsection{Introduction}

On June 23, 1961 Robert M. White flew the North American X-15 at Mach 5.3, becoming the first pilot to achieve hypersonic flight [1]. It is astounding that this milestone occurred only 57 years after the Wright Brothers' first flight. Fifty five years have passed since that historic day in 1961, yet sustained hypersonic flight remains an elusive and challenging goal.

Early interest in hypersonics grew out of the necessity of returning astronauts safely back to Earth in the late 1950s [2]. A substantial effort was focused on developing alternatives to rocket- and capsule-based access to space systems with greater range upon reentry (i.e., higher lift-to-drag) and therefore, increased mission flexibility [2]. Specifically, the concept of a reusable, single-stage-to-orbit vehicle that could take off horizontally, accelerate into orbit, reenter the atmosphere, and land on a runway was highly desirable [1]. This desire resulted in several 
research efforts including the Dyna-Soar (X-20) program [3] from 1957 to 1963 and later the National Aerospace Plane (NASP) program [4] from 1983 to 1995. Each program sought to develop a reusable, manned hypersonic vehicle capable of reaching low earth orbit, using either a boost-glide system [3] or an air-breathing engine [4]. However, due to overambitious goals the design requirements for these programs often exceeded the state-of-the-art, ultimately resulting in their cancellation.

More recent efforts have scaled back the lofty objectives of these earlier programs to explore specific technical aspects of hypersonic flight. In 1996, NASA initiated the Hyper-X program to flight-demonstrate an airframe-integrated scramjet propulsion system operating between Mach 5 and 10 [2]. This program culminated in the first successful scramjet powered flight in 2004, in which the X-43A achieved Mach numbers of 6.8 and 9.6 [5]. This was followed by the X-51A Waverider Scramjet Engine Demonstrator program that aimed to develop technologies for scramjet engines using endothermic hydrocarbon fuel [6]. In 2013, the X-51A sustained speeds exceeding Mach 5 for 210 seconds, setting the record for longest air-breathing hypersonic flight [6]. In addition to these technology demonstrators, the Hypersonic International Flight Research and Experimentation (HIFiRE) program was established in 2006, with the goal of gathering flight test data to improve our understanding of fundamental hypersonic phenomena [7]. These recent successes have rekindled the United States' interest in developing reusable hypersonic vehicles. 
In particular, the Air Force Research Laboratory (AFRL) seeks to enable the development of a Mach 5 to 7 hypersonic cruise vehicle (HCV) with takeoff weight exceeding 300,000 pounds, an unrefueled range of 2,000 nautical miles, and service life on the order of hundreds to thousands of hours [8-11]. To help satisfy these requirements, the vehicle will use a lifting body configuration with an airframeintegrated propulsion system consisting of a combined cycle (turbojet and dualmode scramjet) engine [11-13]. Additionally, a non-ablative thin-gauge metallic thermal protection system is desired in lieu of ablative ceramic insulation $[8,9]$ for the improved serviceability, durability, and potential weight reduction [14, 15]. Several concepts for future $\mathrm{HCVs}$, proposed by Boeing and DARPA, are provided in Fig. 1.1.

For reusable HCVs, like that in Fig. 1.1, to become a reality significant advancements must be made in the state-of-the-art for predicting the response and life of the structure $[8,9]$. These advancements are driven by the enhanced coupling between the flowfield and the vehicle due to the extreme operating environment associated with hypersonic flight. A major area of concern is the accurate determination of the aerothermodynamic loads, particularly the aerodynamic heating and pressure. The aerodynamic heat load and peak surface temperature affect the material strength and stiffness, result in permanent changes such as creep and plasticity, and are required to compute the internal heat transfer in the vehicle [8]. For high-speed flight regions of high-temperature flow are produced by viscous dissipation in the boundary layer, bow shocks near stagnation regions, and 


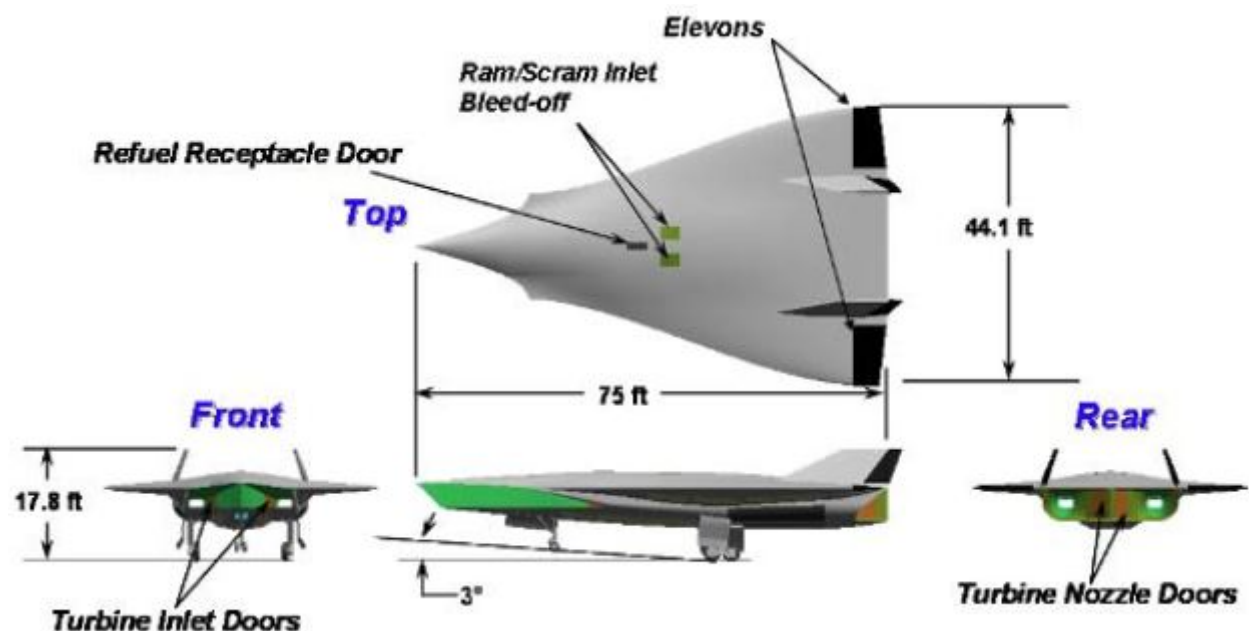

(a) Boeing Manta 2025 [9].

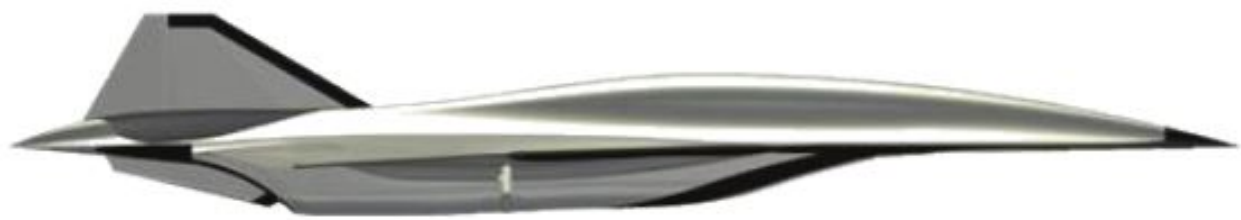

(b) Lockheed concept for DARPA Falcon Program Blackswift/HTV-3X [11].

Figure 1.1: Conceptual hypersonic cruise vehicles. 
oblique shocks that impinge on the vehicle surface (i.e., shock/boundary-layer interactions) [1]. The increased importance of thermal effects at hypersonic speeds is clearly demonstrated in Fig. 1.2, in which an approximate relationship between Mach number and the adiabatic surface temperature [16] is plotted for freestream temperatures that span the operating altitude range of 30 to $80 \mathrm{~km}$. As Mach number increases, high-temperature effects (vibrational excitation, dissociation, and ionization) become more prominent and the flow can potentially alter the structure through ablation and oxidation $[8,12]$. Also, high-frequency pressure fluctuations generated by turbulent motion in the boundary layer, engine excitation noise, and shock/boundary-layer interactions impact the dynamic response [17] and fatigue life of vehicle structures [18].

During atmospheric flight $\mathrm{HCV}$ s are primarily subject to fluid loading in which the flowfield is transitioning from a laminar to a turbulent state [12]. This is a result of the boundary layer becoming more stable as flight speeds exceed Mach 4 [19] and the large trip size required to force transition in hypersonic flight [20]. While there is large uncertainty associated with the length of transition at hypersonic speeds, previous studies have observed transition lengths equal to or greater than the extent of the laminar region [21,22]. Therefore, the boundary layer may be transitional over the majority of a HCV. Aerodynamic heating and boundarylayer pressure fluctuations vary significantly with the state of the boundary layer (laminar, transitional, or turbulent). For instance, heat transfer rates for turbulent boundary layers are three to eight times greater than laminar conditions [23]. 


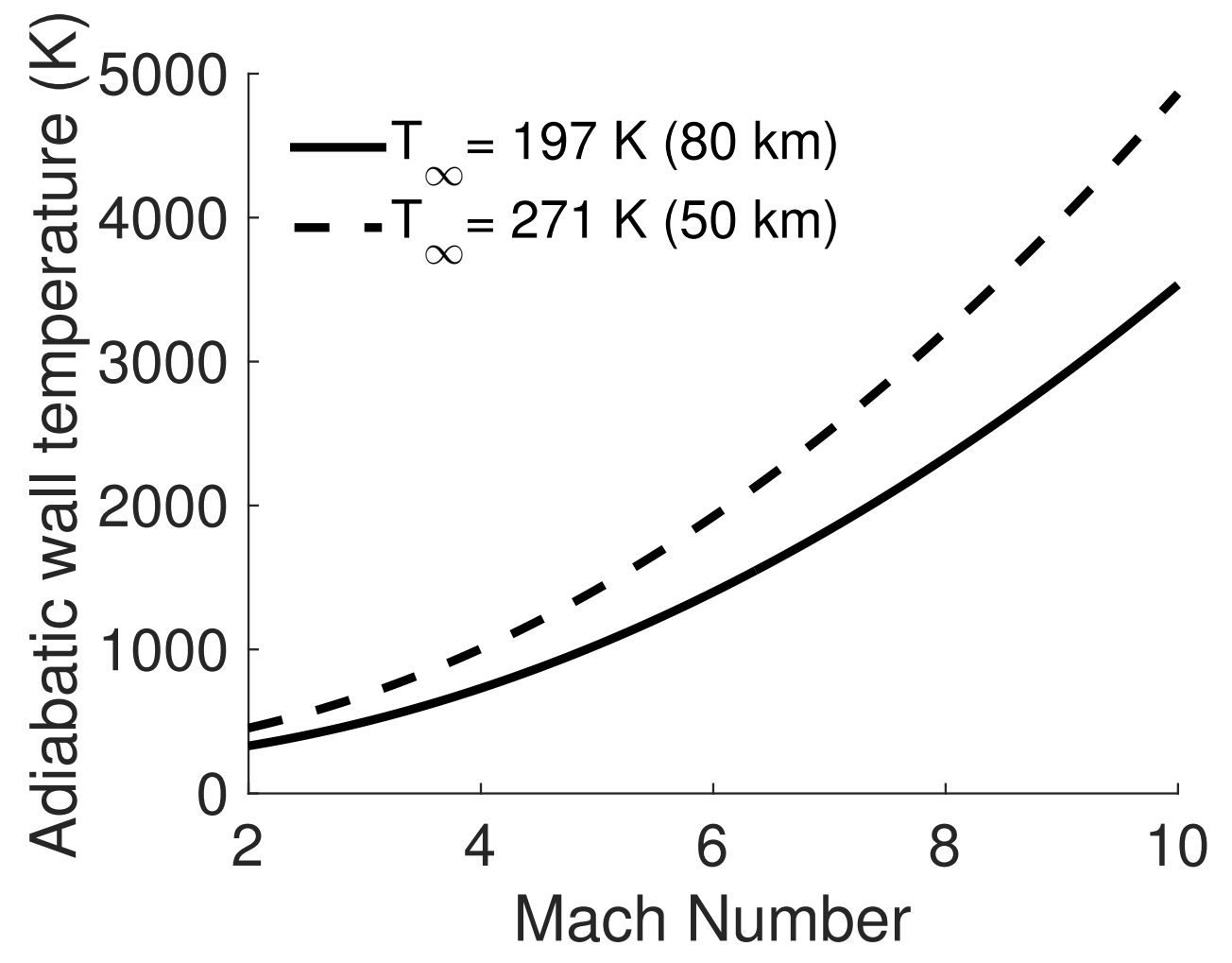

Figure 1.2: Dependence of adiabatic wall temperature on the freestream Mach number. 
Furthermore, during transition heating rates and fluctuating pressure loads may exceed, or overshoot, the fully turbulent values [22, 24-29]. Previous work has found that transitional aerodynamic heating alters the natural frequencies of vehicle structures, producing responses that are not bounded by laminar and turbulent loading conditions [30]. Therefore, boundary-layer transition must be accounted for in order to accurately predict the aerothermal environment and the response of the structure.

The severe loading (thermal, acoustic, and mechanical) associated with hypersonic flight necessitates improvements in airframe structural efficiency through the use of unique and minimum-weight concepts [8]. However, the compliant nature of these structures, in combination with the severe aerothermodynamic loads, results in a propensity for fluid-thermal-structural interactions (FTSI). The coupling associated with FTSI, or aerothermoelastic (ATE) interactions, is schematically depicted in Fig. 1.3. The fluid interacts with the structure through the application of aerothermodynamic loads, in the form of aerodynamic pressure $(P)$ and heat flux $(Q)$. These loads determine the structural response, which is coupled back to the fluid through the structural dynamics $(w, \dot{w})$ and surface temperature $\left(T_{w}\right)$. There is also thermo-elastic coupling within the structure as the temperature field $(T)$ alters the structural mechanics.

Hypersonic FTSI, or aerothermoelasticity, has been an intermittent area of research since the late 1950s. Today it remains a challenging area of study due to: the inability to adequately replicate flight conditions in ground based facilities; 


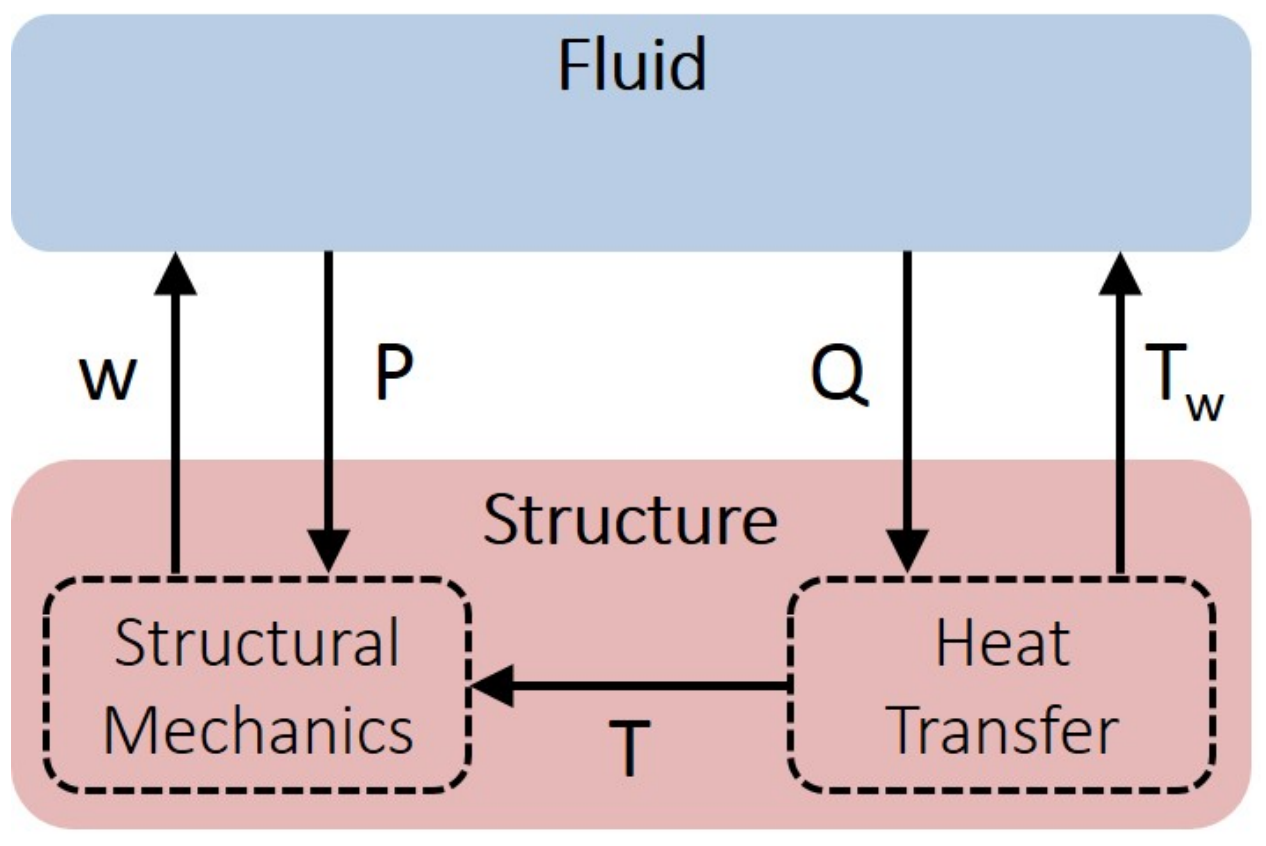

Figure 1.3: Schematic of fluid-thermal-structural interaction.

the impracticality of extensive flight testing; and the intractability of tightly integrated, computational analysis using state-of-the-art tools in all the relevant disciplines $[2,8-11,31]$. Thus, current efforts are focused on developing multidisciplinary models that capture the relevant physics and the required coupling between the individual disciplines (fluid, thermal, and structural) [17, 32-38]. Previous studies on the aerothermoelastic response of hot structures indicate that peak deformation may be on the same order as the thickness of the boundary layer $[33,35,39,40]$, are potentially transient in nature due to fluctuating pressures from the flow $[32,35,41]$, and lead to increased spatial variation in the surface temperature $[33,39,40]$. While these studies have advanced the state-of-the-art in tractable multidisciplinary analysis, they do not consider how the structural response im- 
pacts boundary-layer stability.

Boundary-layer stability is highly dependent on wall temperature $[42,43]$ and surface geometry [43-53], both of which vary during flight for hot structure hypersonic vehicles. As the vehicle response alters the boundary-layer state, this in turn affects the aerothermodynamic loads acting on the vehicle. The role of transition on aerothermodynamic loading, combined with the mutual dependence of boundary-layer stability and the surface conditions, make the potential for interaction between the structural response and boundary-layer transition an important and challenging area of study.

\subsection{Literature Review}

This dissertation focuses on the interaction between boundary-layer transition and the aerothermoelastic response of surface panels on hypersonic vehicles. In order to establish the state-of-the-art for this problem, the following areas are reviewed: (1) boundary-layer stability analysis and transition prediction, (2) physical parameters that alter the stability of hypersonic boundary layers, (3) aerothermoelastic simulation of structures in hypersonic flow, and (4) consideration of transitional boundary layer aerothermodynamic loading for structural response prediction. 


\subsubsection{Boundary-Layer Stability and Transition}

\subsubsection{History and Overview}

The study of a fluid's tendency to transition from a laminar to a turbulent state traces back to the experiments of Osborne Reynolds [54] in the late 19th century. In 1880, Reynolds examined the motion of water through glass pipes of various diameter using streaks of dye. By increasing the velocity at a constant diameter, Reynolds observed the streak transitioned from a straight line to a sinuous or turbulent state above a definite value of a nondimensional grouping of the kinematic viscosity, fluid velocity, and a length scale given by the pipe diameter (i.e., the Reynolds number, as coined by Sommerfeld in 1908 [55]). Furthermore, Reynolds remarked that the critical velocity, or Reynolds number, at which the flow became turbulent was highly dependent on the disturbance environment at the pipe inlet and irregularities in the surface of the pipe [54]. This observation led Reynolds to the hypothesis that, "disturbances...were necessary to the existence of a state of instability [54]" and established the notion that transition to turbulence can be viewed as a fluid stability problem.

The first major breakthrough in boundary-layer stability theory was achieved in the 1930s by Walter Tollmien and Hermann Schlichting who provided a theoretical calculation of the indifference Reynolds number. The theoretical approach of Tollmien and Schlichting tracks the rate of change of small disturbances that are su- 
perimposed on a laminar boundary layer [56]. The indifference Reynolds number delineates the regions of stable and unstable disturbance growth, where the flow will remain laminar for Reynolds numbers below the indifference value. In 1943, Schubauer and Skramstad [57] experimentally confirmed the theory of boundarylayer stability through examination of transitional flow over a flat plate in the 4.5 $\mathrm{ft}$. wind tunnel at the National Bureau of Standards. For part of the experiment, fixed-frequency disturbances were imposed in the boundary layer through forcing a metal ribbon to oscillate using an electromagnet. A hot-wire anemometer was used to see if the forced perturbations persisted downstream. By increasing the frequency of the ribbon, Schubauer and Skramstad [57] were able to measure where the imposed perturbation first appeared and disappeared at the downstream hotwire, and in this manner were able to compute the lower and upper branches of the neutral stability curve. The theoretical agreement was made possible by the low turbulence intensity (approximately $0.02 \%$ ) of the wind tunnel. The turbulence intensity $(T u)$ is defined in Eq. (1.1) as the root mean square of the velocity fluctuations $\left(u^{\prime}, v^{\prime}, w^{\prime}\right)$ divided by the freestream velocity $(U)$.

$$
T u=\frac{\sqrt{\frac{1}{3}\left(u^{\prime 2}+v^{\prime 2}+w^{\prime 2}\right)}}{U}
$$

An idealized representation of the different stages of the transition process is provided in [58] and repeated here in Fig. 1.4 for quiet, incompressible boundarylayer flow over a flat plate. The boundary layer is initially stable and laminar near 
the leading edge of the plate. Through the process of receptivity [59], external disturbances in the freestream (vortical, acoustic, or entropic) enter the boundary layer and excite boundary-layer modes, such as 2-D Tollmien-Schlichting instabilities $(T / S$ waves). Further downstream the laminar flow becomes unstable as the Tollmien-Schlichting instabilities begin to grow. Note that this stage of transition corresponds with the output of boundary-layer stability theory in which the minimum Reynolds number for unstable disturbances $\left(R e_{c r i t}\right)$ is identified. As the Tollmien-Schlichting waves grow they exhibit spanwise variation that results in spanwise vorticity and eventually 3-D vortex breakdown. The final stage of transition is the generation of turbulent spots, that spread and interact as they convect downstream, eventually resulting in a fully turbulent boundary layer. The illustration in Fig. 1.4 highlights the distinction between stability theory and transition prediction as the boundary layer does not exhibit turbulent characteristics until a finite distance downstream of $R e_{\text {crit }}$. However, as the initial departure from the steady, laminar flow begins with the unstable growth of small disturbances, correlations have been developed to relate the growth of disturbances to the onset of transition. Different approaches for the assessment of boundary-layer stability and the prediction of transition are discussed in Sections 1.2.1.2 and 1.2.1.3.

\subsubsection{Boundary-Layer Stability Analysis}

Stability analysis determines whether a boundary layer can remain laminar when subject to a small perturbation. In other words, will the perturbation grow or de- 

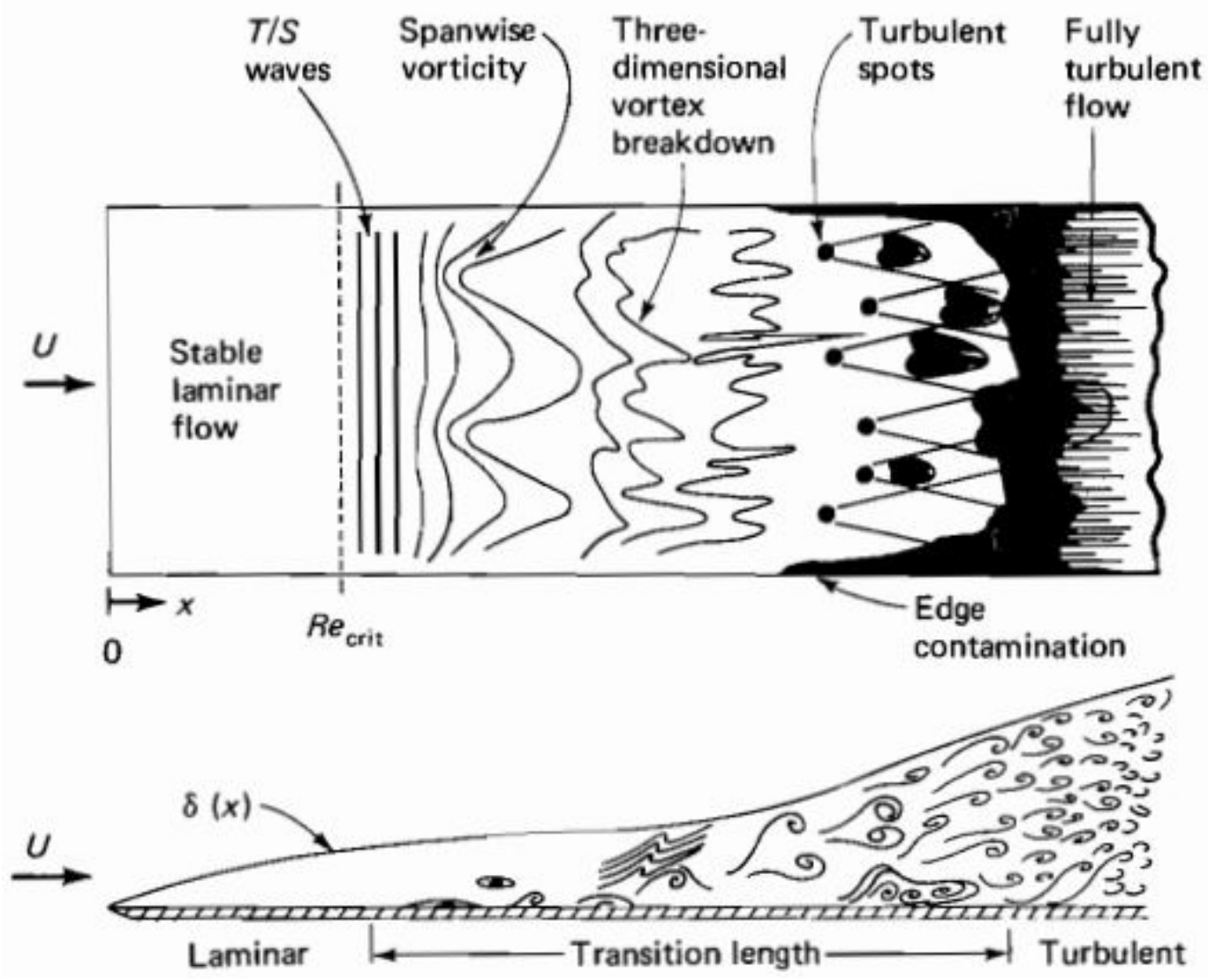

Figure 1.4: Boundary-layer transition process (figure 5-28.a of [58]). 
cay either spatially or temporally? The general procedure required to formulate the stability theory equations is independent of the flow complexity. Therefore, the methodology will be briefly described, following the derivation in [16], for the simplifying case of 2-D incompressible, viscous laminar flow with constant properties. First, the flow variables are decomposed as the sum of a mean component and a small perturbation. The mean terms correspond to a solution of the NavierStokes equations, for which the stability is being assessed. Assuming the only nonzero component of the mean flow velocity field is $U=U(y)$, the flow variables are decomposed as:

$$
\begin{aligned}
& U=\bar{U}+u \\
& V=v \\
& P=\bar{P}+p
\end{aligned}
$$

where barred terms denote the mean flow and lower case terms are the perturbations. The expressions in Eq. (1.2) are substituted into the Navier-Stokes equations and terms that are nonlinear with respect to the perturbations are neglected as they themselves are assumed to be small. Additionally, terms involving only the mean flow variables can be neglected as the mean flow is a solution of the Navier-Stokes equations. Neglecting the above terms results in a linearized form of the continuity 
and $\mathrm{x}$ - and $\mathrm{y}$-momentum for the disturbance quantities $(u, v, p)$ :

$$
\begin{gathered}
\frac{\partial u}{\partial x}+\frac{\partial v}{\partial y}=0 \\
\frac{\partial u}{\partial t}+\bar{U} \frac{\partial u}{\partial x}+v \frac{\partial \bar{U}}{\partial y}+\frac{1}{\rho} \frac{\partial p}{\partial x}=\nu \nabla^{2} u \\
\frac{\partial v}{\partial t}+\bar{U} \frac{\partial v}{\partial x}+\frac{1}{\rho} \frac{\partial p}{\partial y}=\nu \nabla^{2} v
\end{gathered}
$$

The linearized disturbance equations [Eqs. (1.3)-(1.5)] are simplified by assuming the perturbations are traveling waves. For this case the velocity perturbations are described through a stream function $(\psi)$ to reduce the number of unknowns:

$$
\psi(x, y, t)=\phi(y) e^{i(\alpha x-\beta t)}
$$

where $\phi(y)$ is a shape function defining the initial amplitude of the disturbance, $\alpha$ is the wavenumber, and $\beta$ is the frequency. For 2-D parallel flow, Squire's theorem states that the lowest unstable Reynolds number corresponds to a 2-D disturbance propagating in the same direction as the base flow [58]. Representing the velocity perturbations through the stream function and eliminating the pressure terms in the $\mathrm{x}$ - and $\mathrm{y}$-momentum equations reduces the disturbance equations to a fourthorder ODE for the shape function $\phi(y)$.

$$
(\bar{U}-c)\left(\phi^{\prime \prime}-\alpha^{2} \phi\right)-\bar{U}^{\prime \prime} \phi=-\frac{i}{\alpha R e}\left(\phi^{\prime \prime \prime \prime}-2 \alpha^{2} \phi^{\prime \prime}+\alpha^{4} \phi\right)
$$


The velocity perturbations are assumed to vanish at infinity and at the wall to satisfy the no slip condition. Therefore, the boundary conditions require that $\phi=0$ and $\phi^{\prime}=0$ at both the wall and the edge of the boundary layer. The expression in Eq. (1.7), known as the Orr-Sommerfeld equation, is an eigenvalue problem. The eigenfunction is the disturbance amplitude function $\phi(y)$. The eigenvalue is either the disturbance wavenumber $(\alpha)$ or velocity $(c=\beta / \alpha)$ depending on whether the spatial or temporal stability is being examined. For spatial stability, $\alpha$ is complex where the real component corresponds to the wavenumber and the imaginary component is an amplificaiton/damping coefficient. Note that if the viscous terms in Eq. (1.7) are neglected the expression reduces to the Rayleigh equation:

$$
\phi^{\prime \prime}-\left(\frac{\bar{U}^{\prime \prime}}{\bar{U}-c}+\alpha^{2}\right) \phi=0
$$

Through examination of Eq. (1.8) Lord Rayleigh determined that velocity profiles with a point of inflection are unstable [16]. An example of a velocity profile that could have an inflection point is a boundary layer with an adverse pressure gradient.

The preceding derivation of the Orr-Sommerfeld equation highlights the major steps of any boundary-layer stability analysis: (1) the flow variables are decomposed as a mean and perturbation term, (2) the decomposed variables are substituted into the Navier-Stokes to obtain equations for the disturbances, (3) the disturbance equations are often linearized, (4) the disturbance equations are sim- 
plified by assuming the form of the disturbances, and (5) the resulting equations are solved to assess the stability of the mean flow. Despite this common procedure, different methodologies exist to assess boundary-layer stability. The various approaches can be classified according to the assumptions made regarding the dimension of the mean flow and the form of the disturbances. Juniper et al. [60] provides a comparison of local, nonlocal, and global approaches that is repeated here in Tables 1.1 and 1.2. Each approach assumes the flow variables $\vec{q}=(\rho, u, v, w, T)^{T}$ are decomposed as:

$$
\vec{q}=\bar{q}+\epsilon \hat{q} e^{i \theta}
$$

where $\bar{q}$ defines the mean flow, $\epsilon \hat{q} e^{i \theta}$ is the small perturbation, and $\theta$ is a phase function that characterizes the wavelike nature of the disturbance. The approaches compared in Tables 1.1 and 1.2 include Linear Stability Theory (LST), the Parabolized Stability Equations (PSE), Biglobal analysis, PSE-3D, and Triglobal analysis. Table 1.1 lists the assumptions and form of the mean flow, while Table 1.2 provides the form of the assumed perturbation. The methods are listed in order of decreasing assumptions and increasing computational expense.

The simplest and most dimensionally restrictive approach is LST, which assumes the mean flow is quasi-parallel. As shown in Table 1.1, LST assumes the streamwise and spanwise derivatives of the mean flow $\left(\bar{q}_{x}, \bar{q}_{z}\right)$ are zero. In doing so the mean-flow quantities that arise from the growth of the boundary layer are neglected and the disturbance characteristics are determined by local boundary- 
Table 1.1: Basic state assumptions of linear stability methods (adapted from [60]).

\begin{tabular}{lcc}
\hline \hline Method & Assumption & Basic State \\
\hline LST & $\bar{q}_{x}=0, \bar{q}_{z}=0$ & $\bar{q}(y)$ \\
PSE $^{\mathrm{a}}$ & $\bar{q}_{x} \ll \bar{q}_{y}, \bar{q}_{z}=0$ & $\bar{q}\left(x^{*}, y\right)$ \\
Biglobal & $\bar{q}_{x}=0$ & $\bar{q}(y, z)$ \\
PSE-3D & $\bar{q}_{x} \ll \bar{q}_{y, z}$ & $\bar{q}\left(x^{*}, y, z\right)$ \\
Triglobal & none & $\bar{q}(x, y, z)$ \\
\hline \hline
\end{tabular}

a $x^{*}$ denotes slow variation in the $x$ or streamwise direction.

Table 1.2: Perturbation assumptions of linear stability methods (adapted from [60]).

\begin{tabular}{lcc}
\hline \hline Method & Amplitude Function & Phase Function $\theta$ \\
\hline LST & $\hat{q}(y)$ & $\alpha x+\beta z-\omega t$ \\
PSE & $\hat{q}\left(x^{*}, y\right)$ & $\int \alpha\left(x^{*}\right) d x^{*}+\beta z-\omega t$ \\
Biglobal & $\hat{q}(y, z)$ & $\alpha x-\omega t$ \\
PSE-3D & $\hat{q}\left(x^{*}, y, z\right)$ & $\int \alpha\left(x^{*}\right) d x^{*}-\omega t$ \\
Triglobal & $\hat{q}(x, y, z)$ & $\omega t$ \\
\hline \hline
\end{tabular}

layer profiles (i.e., local approach). The Orr-Sommerfeld and Rayleigh equations [Eqs. (1.7) and (1.8)] are specific forms of LST. In 1946, Lees and Lin [61] applied an asymptotic form $(R e \rightarrow \infty)$ of LST to investigate the stability of 2-D compressible flow over a flat plate. Lees and Lin [61] demonstrated that for compressible flow Rayleigh's inflection point criterion for inviscid instability is replaced by:

$$
\left[\frac{d}{d y}\left(\rho \frac{d u}{d y}\right)\right]_{y_{s}}=0
$$

The expression in Eq. (1.10), termed the generalized inflection point, reveals that the gradient of the product of density and vorticity is analogous to the curvature 
of the velocity profile for incompressible flow. Applying the chain rule to Eq. (1.10) indicates that compressible boundary layers may be inviscidly unstable in the absence of an inflected velocity profile if the wall is cooled (i.e., $\rho_{y}<0$ ).

In the late 1960s, Mack [62] numerically solved the LST equations for a compressible boundary layer. The extensive analysis of Mack revealed additional differences between the stability of incompressible and compressible flows, which are thoroughly reviewed in $[16,19,63]$. The primary distinction is that when a region of the boundary layer is supersonic, relative to the disturbance phase velocity, there exists an infinite number of neutral waves with different wavenumbers and the same phase velocity. Analytical proof of this behavior can be obtained by observing that the second-order compressible inviscid stability equations for the velocity [64] and pressure $[19,64]$ fluctuation amplitude functions become general wave equations when a region of supersonic relative flow exists. In supersonic and hypersonic flow these instabilities, referred to as the higher-, Mack-, or acousticmodes, coexist with the Tollmien-Schlichting waves (i.e., first-mode instabilities) observed in incompressible flow. The terminology acoustic-modes references the fact that the neutral modes can be visualized as sound waves reflecting between the wall and the sonic line in the boundary layer [63]. The acoustic modes are most unstable as 2-D waves, and unlike the first-mode their stability are not governed by the generalized inflection point [63]. The first acoustic mode (i.e., second mode) becomes the dominant instability for Mach numbers greater than four [44]. In addition to the discovery of the acoustic-modes, Mack found that for supersonic flow 
the first-mode instability is most unstable as an oblique wave oriented at an angle to the freestream [62]. Therefore, Squire's theorem does not extend to compressible boundary layers.

The PSE are a nonlocal approach that can be viewed as an extension of LST for parallel flow with small streamwise variations (e.g., surface curvature). In Tables 1.1 and $1.2 x^{*}$ denotes slow variation in the $x$ or streamwise direction. The PSE method, originally formulated by Bertolotti and Herbert in 1987, consists of a set of nonlinear PDEs that account for mean flow nonparallelism and nonlinearity of the disturbances [65]. The PSE are typically linearized (i.e., LPSE) such that only mean flow nonparallelism is accounted for. Formulations of the LPSE for compressible flow are provided by Bertolotti and Herbert [65] and Chang et al. [66]. As noted by Masad and Abid [67], nonparallel effects are more pronounced on 3-D disturbances. Thus, the LPSE are well suited to assess boundary-layer stability in supersonic flow where the dominant instability is the oblique first-mode.

The biglobal, PSE-3D, and triglobal methods are global approaches that allow for mean flow with multiple inhomogeneous spatial directions [60]. The biglobal method assumes the mean flow only varies in the wall-normal and spanwise directions $(y, z)$. As indicated in Table 1.2, for the biglobal method the mean flow is superimposed with disturbances that are periodic in time and in the streamwise direction. The triglobal method is the least restrictive approach as no assumptions are made regarding the dimensionality of the mean flow. However, the lack of assumptions comes at the cost of increasing the computational expense. Theofilis 
indicates that, in general, the size of the matrices describing the local, biglobal, and triglobal approaches are on the order of $\mathrm{MB}, \mathrm{GB}$, and $\mathrm{TB}$ [68]. In this dissertation, boundary-layer stability is assessed using a compressible formulation of the LPSE as this is the most computationally efficient approach that accounts for streamwise variation in a 2-D mean flow. Derivation of the LPSE used in this work is provided in Section 2.1.2.

\subsubsection{Transition Prediction}

While many methodologies exist to compute the amplification of disturbances in a boundary layer, the prediction of transition remains a difficult task. Stetson [69] summarizes the current state of affairs by stating that, "The relation between boundary-layer stability and transition is not well understood" and "There is no transition theory". For this reason, boundary-layer transition is predicted through empirical or semi-empirical correlations. Empirical methods correlate transition with properties of the flowfield, such as assuming transition occurs at a constant value of $\frac{R e_{\theta}}{M_{e}}$, which was applied in the initial design of the space shuttle [1]. Semiempirical approaches relate transition to the unstable growth of disturbances predicted through boundary-layer stability analysis.

In 1956, Smith and Gamberoni [70] and van Ingen [71] independently developed semi-empirical correlations to predict transition based off the theory of boundary layer stability. Either approach relates transition to the amplification ratio of disturbances in the boundary layer. The method in [71] was developed for the 
temporal stability of incompressible flow, where a 2-D periodic disturbance can be expressed through its stream function as:

$$
\psi=\phi(y) e^{\beta_{i} t} e^{i\left(\alpha x-\beta_{r} t\right)}
$$

In Eq. (1.11) the disturbance shape function is given by $\phi(y), \alpha$ is the angular wavenumber, and $\beta$ is a complex number whose real and imaginary components correspond to the angular frequency $\left(\beta_{r}\right)$ and the amplificaiton/damping coefficient $\left(\beta_{i}\right)$, respectively. The change in disturbance amplification, or amplification ratio $\left(\frac{A}{A_{o}}\right)$, between the time corresponding to neutral stability $\left(t_{o}\right)$ and some later time is given as:

$$
\frac{A}{A_{o}}=e^{\int_{t_{o}}^{t} \beta_{i} d t}
$$

where the exponent is the amplification factor, commonly referred to as the $N$ factor. Through comparison of stability theory and experimental data for 2-D incompressible flow over a flat plate, van Ingen found that transition onset occurred at an amplification factor of 7 to 8 and ended at 10 [71]. Jaffe et al. [72] modified this approach for spatial stability analysis and found that a $N$ factor of 10 provided good agreement with transition observed in both wind-tunnel and flight tests, as shown in Fig. 1.5. It is interesting that Schlichting correctly conjectured that the integrated disturbance amplification would be an essential value to the transition process [56]. 


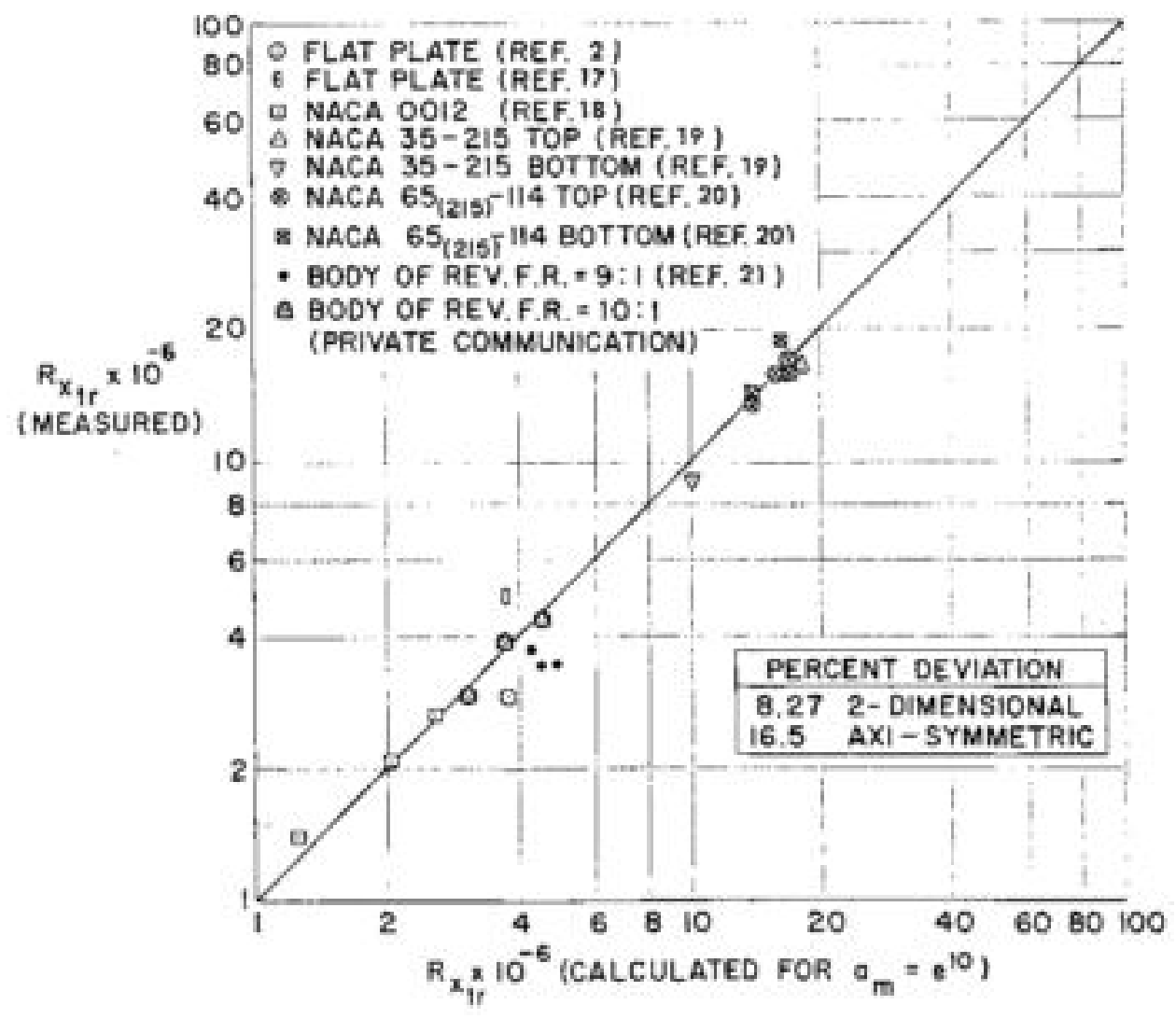

Figure 1.5: Agreement of $e^{10}$ predicted transitional Reynolds number with experimental data (figure 13 of [72]). 
Despite the general agreement in Fig. 1.5, Jaffe et al. [72] indicate that for a majority of the examined data the transitional $N$ factor varies from 8 to 11 . They correctly predict that the variation in $N$ at transition is related to the turbulence level of the incoming flow. Mack [73] proposed that the $e^{10}$ correlation should be modified to a more general form (i.e., the $e^{N}$ method) where the amplification factor $N$ varies as a function of the turbulence intensity. Using flat plate wind-tunnel data, Mack developed the correlation in Eq. (1.13), which is valid for turbulence intensities of 0.07 to 2.98 percent. Mack's relation in Eq. (1.13) predicts a transitional $N$ factor of 5.5 at $T u=0.3 \%$. As the turbulence intensity decreases the transitional $N$ factor increases, achieving a value of 9 for $T u=0.07 \%$.

$$
N \approx-8.43-2.4 \ln T u
$$

The increase in $N$ factor with turbulence intensity is a product of larger amplitude disturbances entering the boundary layer, and therefore less amplification is required promote transition. Mack [19] has shown that if the external disturbance environment can be well characterized an amplitude-based transition criterion should be used instead of an amplification-based approach. The amplitudebased approach assumes transition occurs once a critical disturbance amplitude is achieved. The amplification-based transition correlation of $\frac{A}{A_{o}}$ equal to a constant can be interpreted as an amplitude-based approach if the neutral disturbance amplitude $\left(A_{o}\right)$ is known. Using this method, Mack accurately computed exper- 
imental trends in the transitional Reynolds number for Mach number and wall temperature variations by accounting for wind-tunnel noise levels [19].

\subsubsection{Physical Parameters that affect Hypersonic Boundary-Layer Stability}

Numerous factors influence the transition of a laminar boundary layer to a turbulent state. An exhaustive list is provided by Stetson [69] and repeated in [1]. Several of the parameters include Mach number, freestream turbulence intensity, characteristic shape of the body (e.g., planar or conical), angle of attack, mass injection/removal, surface roughness, pressure gradient, and nose bluntness. A brief overview of how these parameters affect the stability of hypersonic boundary layers is given next.

The results in Fig. 1.6 demonstrate the effect of Mach number on boundarylayer stability and transition. Maximum spatial amplification rates are shown for the oblique first-mode and the 2-D second-mode in Fig. 1.6.a. The amplification rates indicate that the first-mode instability is stabilized as the Mach number increases. However, near Mach 3.7 the second-mode becomes unstable and remains the most dominant instability mechanism at higher Mach numbers. Thus, for compressible boundary layers the prominent instability mechanism varies with Mach number and can loosely be categorized with the distinction between supersonic and hypersonic flow. The effect of Mach number on the Reynolds number at tran- 
sition $\left(R e_{x, t}\right)$ is shown in Fig. 1.6.b, where the dashed lines are curve fits of the free-flight data (shown as markers) at different unit Reynolds numbers and the solid line is a correlation based off wind tunnel data [74]. Both the wind tunnel correlation and the free-flight data indicate that $R e_{x, t}$ initially decreases with Mach number and achieves a minimum near Mach 3 to 4 . Beyond this point $R e_{x, t}$ increases with Mach number (i.e., transition moves downstream). The results in Fig. 1.6.b also highlight the effect of the freestream turbulence intensity or flight environment on transition. Note that the flight data generally predicts greater $R e_{x, t}$ than the wind tunnel correlation at the same Mach number. Traditional (non-quiet) wind tunnels typically predict lower transitional Reynolds numbers than that seen in flight due to the acoustic noise generated by the turbulent boundary layer on the nozzle wall.

Experiments on sharp cones have shown that as the angle of attack increases transition moves rearward on the windward side and forward on the leeward side [75]. This finding is counterintuitive as increasing the angle of attack decreases the Mach number on the windward side of cone which based off results at zero angle of attack should move transition forward. As with incompressible flow, first-mode instabilities are stabilized by favorable pressure gradients and destabilized by adverse pressure gradients [75]. This is a result of the dependence on the generalized inflection point and the fuller velocity profile produced by favorable pressure gradients. The experiments by Kimmel [76] in the Arnold Engineering Development Center (AEDC) Wind Tunnel B facility at Mach 8 indicate the same trend is true for 


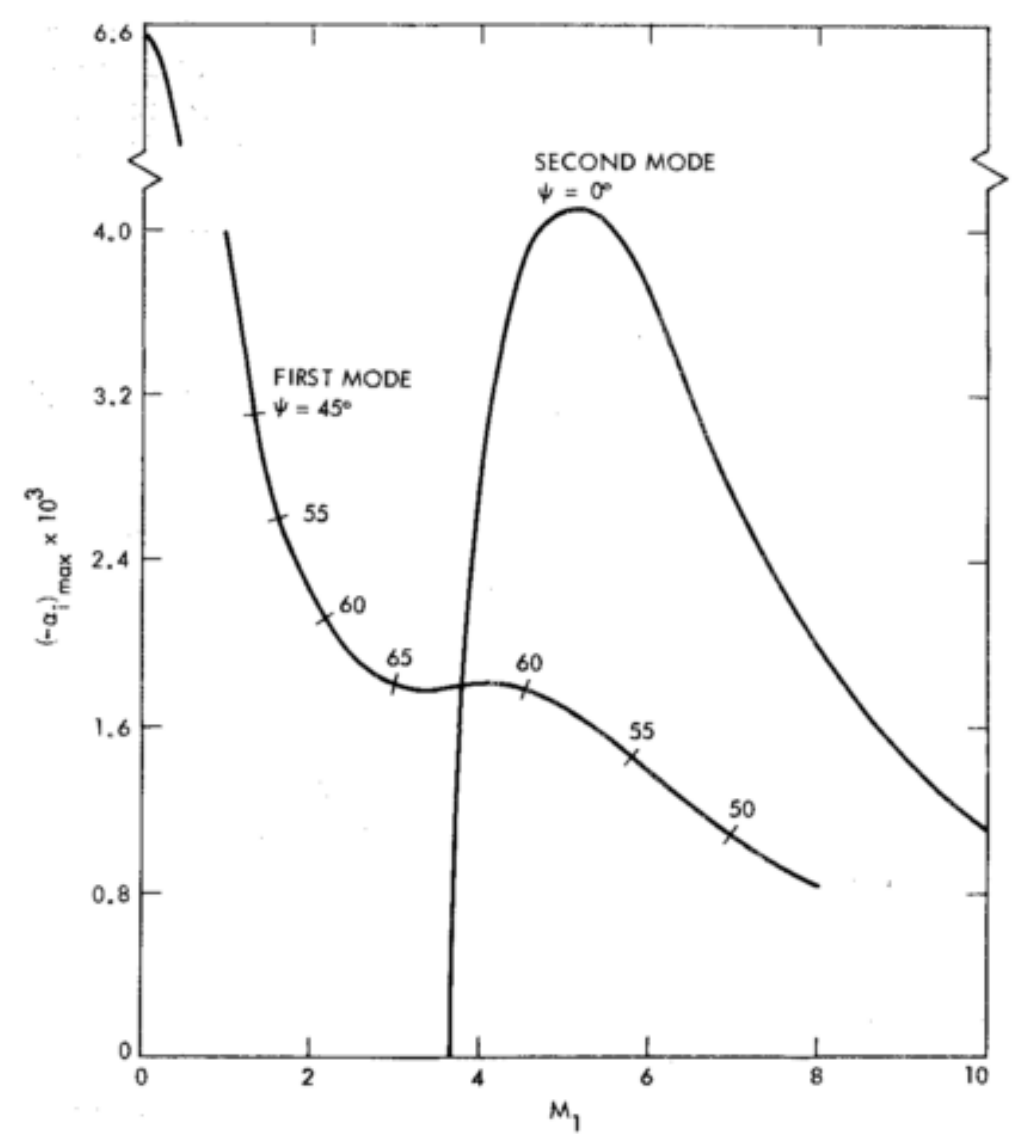

(a) Maximum spatial amplification rate (figure 2 of [19]).

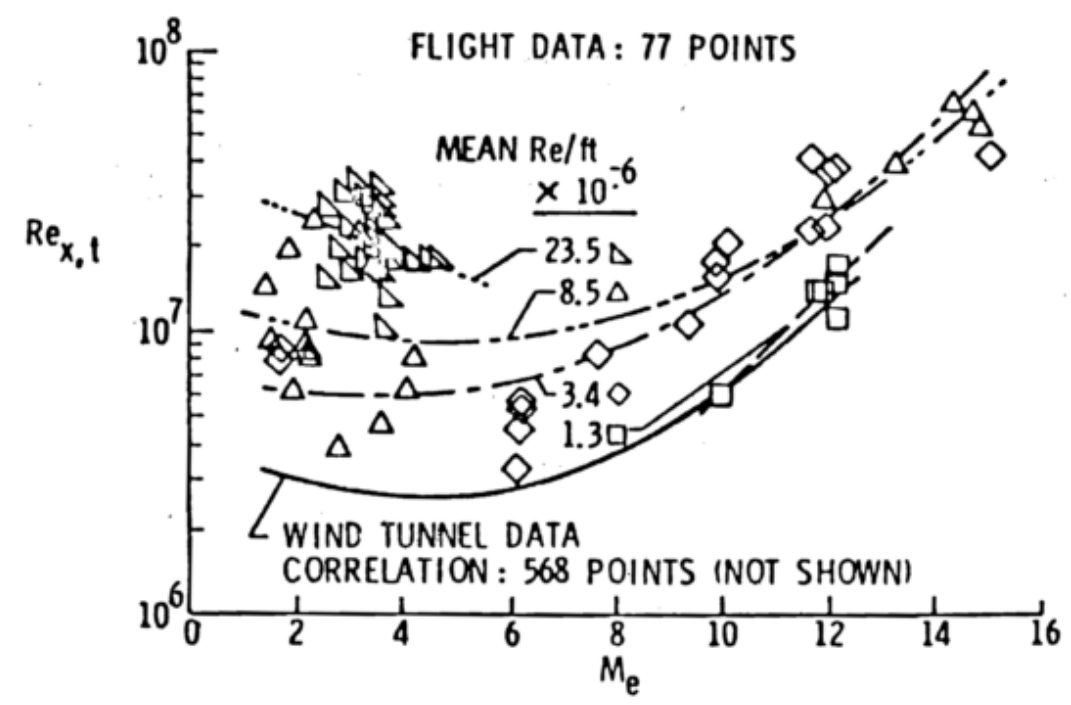

(b) Transition Reynolds number (figure 4 of [74]).

Figure 1.6: Effect of Mach number on hypersonic boundary-layer stability and transition. 
second-mode instabilities. Kimmel [76] performed experiments on a $7^{\circ}$ half-angle, sharp cone with interchangeable flared or ogive aft-bodies to examine the effect of favorable and adverse pressure gradients on transition length. While favorable pressure gradients delayed transition onset, the length of the transition region decreased compared to a cone with no pressure gradient. Nosetip bluntness has an interesting effect of transition, commonly referred to as the "Blunt Body Paradox". In contrast to a sharp cone, small bluntness increases the transitional Reynolds number while large bluntness decreases it [75]. As pointed out by Anderson [1], the large bluntness creates a favorable pressure gradient that typically enhances boundary-layer stability. The destabilizing effect of large bluntness is related to the entropy layer behind the bow shock and highlights the complexity of transition prediction for compressible flow.

Of particular interest to this work is the effect of surface temperature, body curvature, and surface roughness. The first two are important as a hypersonic vehicle will heat up during flight and the surface panels will deform, altering the shape of the structure. Insight into the effect of panel-scale deformations on boundarylayer stability may be gained by previous work examining surface roughness if the height of the surface perturbations have equivalent scales, relative to the boundarylayer thickness. For this reason, the subsequent sections further examine the influence of surface temperature variations (Section 1.2.2.1) and surface perturbations (Section 1.2.2.2) on hypersonic boundary-layer stability. 


\subsubsection{Surface Temperature}

The effect of surface temperature on the stability of hypersonic boundary layers has been extensively examined in previous research and can broadly be categorized into two areas: uniform temperature changes and localized temperature perturbations. For incompressible flow uniform temperature changes alter the boundary-layer stability through the point of inflection criterion, which asserts that velocity profiles with an inflection point are unstable. A point of inflection was proven to be a necessary condition for instability by Lord Rayleigh in the late 19th century and a sufficient condition for disturbance amplification by Tollmien in 1936 [16]. As inflected profiles are unstable, increasing the curvature of the velocity profile at the wall (i.e., making the boundary layer fuller) improves stability. An expression for the curvature of the velocity profile at the wall of an impermeable flat plate is provided in Eq. (1.14) assuming incompressible flow.

$$
\left.\frac{d^{2} u}{d y^{2}}\right|_{w}=-\frac{1}{\mu_{w}}\left(\left.\left.\frac{d \mu}{d T} \frac{d T}{d y}\right|_{w} \frac{d u}{d y}\right|_{w}\right)
$$

Making the curvature at the wall more negative coincides with a fuller, more stable boundary layer. For gas in which viscosity increases with temperature the curvature becomes more negative as the wall is cooled $\left(\left.\frac{d T}{d y}\right|_{w}>0\right)$.

For compressible flow wall cooling stabilizes 2-D and oblique first-mode instabilities. This was shown theoretically by Lees [77] through the asymptotic behavior 
of 2-D disturbances at large Reynolds numbers and later by Mack $[19,62]$ through numerical solution of the LST equations, assuming both 2-D and 3-D sinusoidal disturbances. The stabilizing effect of wall cooling on a compressible boundary layer seems intuitive as it increases the density, which thins the boundary layer and makes the velocity profile fuller [67]. However, the acoustic modes are destabilized by wall cooling [62]. Unlike the first mode, instability of the higher modes does not require a generalized inflection point, only a region of supersonic flow [19]. Therefore, techniques to improve stability through making the velocity profile fuller (e.g., favorable pressure gradients or suction) cannot be expected to stabilize Mack-mode instabilities. The first experimental evidence of the destabilizing effect of wall cooling on a hypersonic boundary layer was provided by Demetriades [78] for Mach 8 flow past a $5^{\circ}$ half-angle cone in the AEDC Wind Tunnel B facility. In 1992, Stetson and Kimmel [79] also observed the destabilization of second-mode waves and the stabilization of first-mode waves with wall cooling.

The impact of wall cooling on the predicted transition onset location for a hypersonic boundary layer is depicted in Fig. 1.7 in terms of the Reynolds number corresponding to $N=9$. The results in Fig. 1.7 clearly show that the oblique firstmode is stabilized and the 2-D second-mode is destabilized as the wall temperature decreases. The red arrows highlight the movement of the transition onset as the wall is cooled assuming the first-mode is the dominant transition mechanism at the adiabatic wall temperature. The arrows indicate that as the surface is cooled the transition onset initially moves downstream. However, further cooling 
$\left(T_{w} / T_{a d}<0.75\right)$ amplifies the 2-D second-mode to the extent where it results in transition, causing the onset location to move upstream. The first-mode stabilization and second-mode destabilization provides an explanation for experimental observations of transition reversal with wall cooling [67].

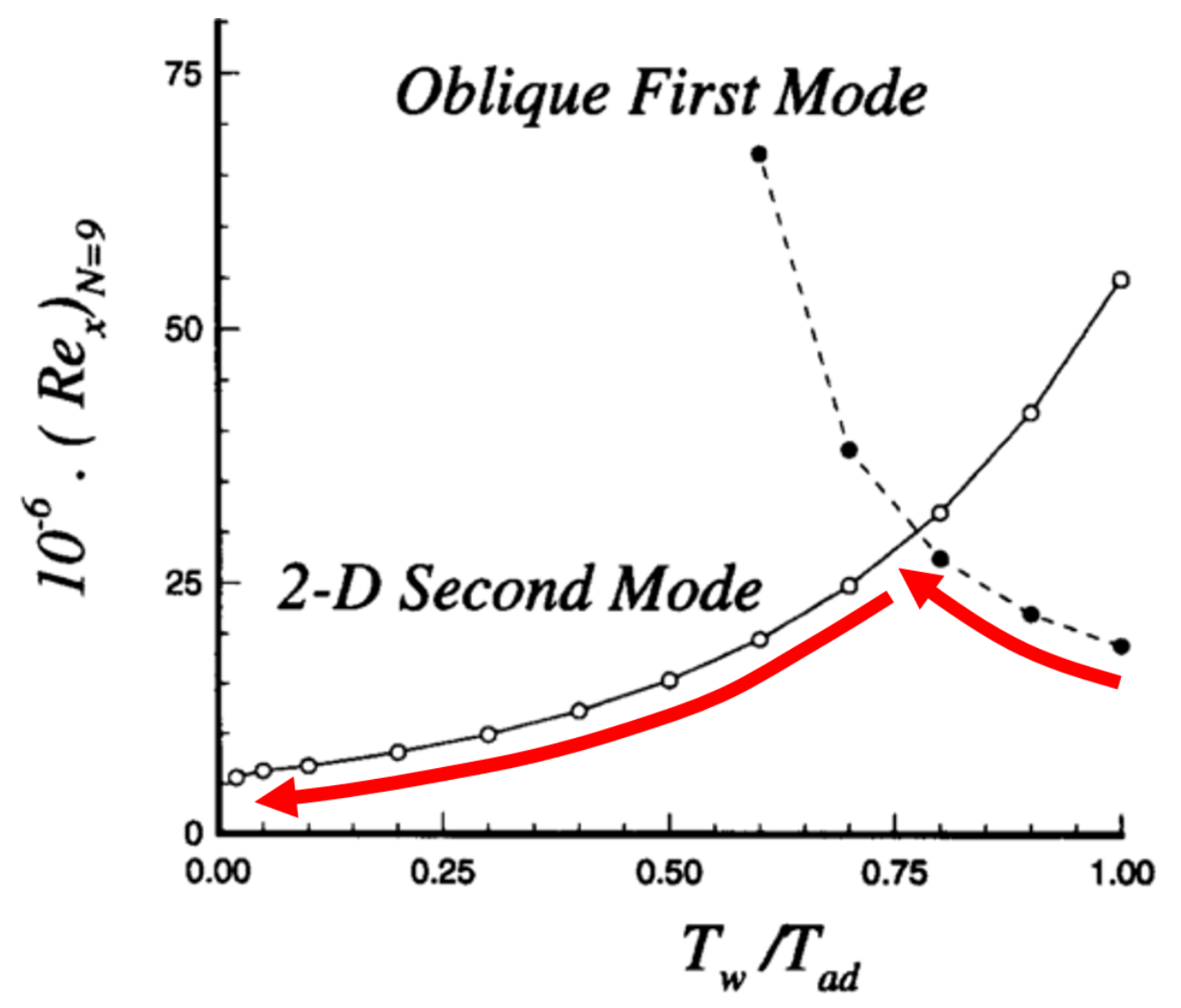

Figure 1.7: Movement of predicted transition location with wall cooling at $M_{\infty}=$ $5, T_{\infty}=300 \mathrm{~K}$ (adapted from figure 11.a of [67]).

In addition to uniform temperature changes, the effect of localized heating and cooling has also been examined. For the application of hypersonic vehicles, localized temperature changes may arise at the interface of TPS elements with different material properties (i.e., conductivity and emissivity). Additionally, aerothermoelastically induced deformation can result in thermal gradients along the surface. 
Therefore, it is important to understand how local changes in the surface temperature can alter the stability of the boundary layer. Previous works by Dovgal et al. [80] and Masad and Nayfeh [81] demonstrate that localized heating strips can stabilize subsonic boundary layers. Note that uniform surface heating produces the opposite effect. Masad and Nayfeh [81] explain that the stabilizing effect of the localized heating is due to the temperature of the fluid increasing over the strip. As the fluid convects downstream of the strip it "sees" a relatively cooler surface, which has a stabilizing effect on Tollmien-Schlichting waves. The stabilizing effect of heating strips extend to compressible boundary layers as first-mode waves are stabilized by wall cooling in the supersonic regime. This was demonstrated in the theoretical work of Masad and Abid [67], who found that placement of a heating strip near the leading edge of a flat plate stabilizes oblique first-mode waves at Mach 3.5. Based on their results, Masad and Abid conjecture that a cooling strip would stabilize the boundary layer of a flow in which transition is dominated by second-mode instabilities [67].

In regard to second-mode instabilities, a recent study by Fedorov et al. [82] examined the effect of localized heating and cooling on boundary-layer stability of Mach 6 flow past a $7^{\circ}$ half-angle, sharp cone at zero angle of attack. The analysis in [82] consists of both experiments, conducted in the Institute of Theoretical and Applied Mechanics (ITAM) Tranzit-M wind tunnel, and numerical computations in the form of LST analysis using the $e^{N}$ method to estimate transition onset and DNS of 2-D disturbances propagating through the boundary layer. The re- 
sults indicate that LST computations of the second-mode amplification rates are in agreement with DNS solutions that account for nonparallel flow effects resulting from the localized temperature variation. Also, localized cooling was found to decrease the second-mode amplitude and delay transition while localized heating had an opposite, and less pronounced, effect [82]. Fedorov et al. [82] conclude that one of the dominant mechanisms leading to this effect is the streamwise alteration of the boundary-layer thickness due to the change in wall temperature. Over the localized region of cooling the density increases, thereby decreasing the boundarylayer thickness. Downstream of the cooling region the boundary-layer thickness rapidly increases and approaches the uncooled distribution. The rapid boundarylayer growth narrows down the instability regions for certain fixed-frequency disturbances, decreasing the distance over which their spatial amplification is integrated, resulting in smaller $N$ factors and delayed transition. Soudakov et al. [83] examined the effect of temperature jumps on the boundary-layer stability of Mach 6 flow over a flat plate using DNS. Their analysis also indicates that the effect of the temperature jump on boundary-layer stability is related to the modification on the boundary-layer thickness. For example, a sudden increase in the wall temperature thickens the boundary layer and creates compression waves. Conversely, a sudden decrease in temperature thins the boundary layer and results in expansion waves. 


\subsubsection{Surface Perturbations}

The effect of surface roughness, in the form of both discrete/isolated and distributed elements, has also been extensively examined in previous works [43, 84, 85]. Isolated roughness includes both 2-D/axisymmetric (i.e., ridges, trenches, and trip wires) and 3-D (i.e., steps, gaps, and rivets) geometries. Distributed roughness typically refers to three-dimensional patterns such as that resulting from ablation or surface finishing [43]. A majority of the research on roughness is focused on examining its potential to serve as a laminar boundary layer trip and determining the maximum allowable roughness height that does not alter the naturally occurring transition onset location [86, 87]. However, several studies suggest that the proper application of roughness can potentially delay transition onset.

The ability to delay boundary-layer transition through surface roughness was observed as early as 1959 by James [88], who reported that for a given Mach number an optimum 2-D distributed roughness height exists that extends the laminar region of the flow beyond that occurring for a smooth geometry. Similarly, Holloway and Sterrett [89] observed a slight delay in transition for spherical roughness elements with height less than the boundary-layer thickness at Mach 6. More recently, Saric has demonstrated the ability to delay transition through carefully designed distributed roughness in both subsonic [90] and supersonic [91] flow.

In regard to hypersonic flow, several recent studies indicate that 2-D roughness (discrete and distributed) may delay transition. Through experimental examina- 
tion of a 2-D wavy-wall roughness pattern in Mach 7.1 flow, Fujii observed delayed transition when the roughness wavelength was roughly equal to that of the most unstable second-mode [48]. Marxen et al. [49] numerically investigated the effect of a 2-D roughness element on disturbance growth at Mach 4.8 and found that, downstream of the roughness element, high frequency second-mode disturbances were stabilized while low frequencies were amplified. A similar effect was reported by Bountin et al. [50] for Mach 6 flow past a 2-D wavy wall with cavity depth on the order of the boundary-layer thickness. Recent work by Duan et al. [51] and Fong et al. [52] at UCLA suggests that the stabilizing or destabilizing influence of an isolated roughness element is related to the synchronization point of the slow (Mode S) and fast (Mode F) disturbance modes. Specifically, when the roughness is placed at the synchronization point of a particular frequency, disturbances at higher frequencies are damped while lower frequencies are amplified. The increased amplification of frequencies lower than that of the synchronization point at the roughness location has recently been observed by Tang et al. [92] for flow past an isolated roughness element in the $120 \mathrm{~mm}$ Mach 6 quiet tunnel at Peking University. Motivated by the theoretical work of Zhong's research group at UCLA [51,52], experiments were carried out on a flared cone with six elliptic roughness strips in the Boeing/AFOSR Mach-6 Quiet Tunnel at Purdue University [53]. The first roughness strip was placed downstream of the synchronization point corresponding to the most unstable disturbance frequency of $285 \mathrm{kHz}$. The experimental results clearly show that, with application of the roughness strips, 
the $285 \mathrm{kHz}$ disturbance is completely damped.

In regard to large-scale surface topology variations, previous studies have examined how aerothermoelastic effects, such as thermally induced deformations, can augment aerothermal loads [40,93] and impact boundary-layer transition [94]. Glass and Hunt $[40,93]$ performed aerothermal tests on rigid, spherical dome protuberances mounted on a flat plate at Mach 6.5 in the NASA Langley $8 \mathrm{ft}$. High Temperature Tunnel. The tests were conducted in order to investigate aerothermal load augmentation for dome protuberances typical of bowed TPS tiles [93]. For a dome height of 0.1 in., representative of a bowed metallic TPS tile, the integrated heat load increased by less than 15 percent [40]. Berry et al. [94] examined how the expected bowing of metallic TPS panels could effect boundary-layer transition on the X-33 flight vehicle. Through wind-tunnel testing of X-33 configurations, Berry et al. [94] concluded that a 3-D array of bowed panels was less effective at forcing transition onset than discrete roughness. In addition, Johnson et al. [95] performed a LPSE-based shape optimization of an axisymmetric slender body and a 3-D blunted delta-body geometry to either delay or promote transition.

\subsubsection{Aerothermoelastic Response of Structures in Hypersonic Flow}

Accounting for aerothermoelastic effects is essential to the design and structural life prediction of hypersonic vehicles. In terms of life prediction, the extreme heating and pressure can lead to bowing or flutter of TPS panels that alters the 
aerothermal loading, thereby changing the expected creep deformation and fatigue life [8]. From a design perspective, the aerothermoelastic response of individual components of a hypersonic vehicle can alter the overall performance due to the tightly-integrated subsystems (e.g., airframe-integrated scramjets). A comprehensive review of the theoretical, computational, and experimental efforts to examine hypersonic aerothermoelasticity is provided by McNamara and Friedmann [31].

\subsubsection{Experiments}

Properly scaling the individual disciplines (fluid, thermal, and structural) makes it challenging to reproduce the aerothermoelastic behavior of hypersonic vehicles in experimental facilities. Dugundji and Calligeros [96] provide the similarity parameters of the aerothermoelastic problem for Mach numbers less than 3.5 and temperatures up to $811 \mathrm{~K}$. Their analysis indicates that if the experimental model is constructed using the vehicle material and air is the test gas similarity is only satisfied for a scaling ratio of one. However, the similarity parameters are less restrictive for simplified experimental models such as the behavior of a wing structure, thin solid plate, and panel flutter [96]. If similarity can not be obtained Dugundji and Calligeros [96] suggest that the aerothermal loads can be estimated in advance and artificially applied to the structure ("incomplete aerothermoelastic" testing), or "restricted purpose" models that examine specific aspects of the aerothermoelastic coupling can be used.

Previous aeroelastic and aerothermoelastic experiments have examined the re- 
sponse of panels in order to satisfy similarity on scaled models. Dixon et al. [97] examined the effect of thermal stress and buckling, induced by aerodynamic heating, on the flutter behavior of skin-stiffened multibay aluminum panels at Mach 3 in the NASA Langley $9 \times 6 \mathrm{ft}$. thermal structures tunnel. The tests were conducted for a range of dynamic pressure (1500-5000 psf), stagnation temperature (300-655 $\left.{ }^{\circ} \mathrm{F}\right)$, and for different panel thickness. The results indicate that as the surface temperature increases, flat panels become more prone to flutter. However, the trend is reversed for thermally buckled panels as further increase in temperature stiffens the panel. In a similar experiment, Guy and Bohon [98] examined the effect of aerodynamic heating on the flutter behavior of aluminum and steel multibay panels, as well as an $\mathrm{X}-15$ vertical-stabilizer panel, in the same facility as [97]. For a flat panel an increase in the thickness is required to prevent flutter with increasing surface temperature. The reverse of this trend is true if the panel is thermally buckled. Interestingly, the flutter boundary of the flat steel panel is largely unaffected by aerodynamic heating. Differences between the aluminum and steel response were attributed to variations in the edge restraints, stress ratios, and pressure differentials on the panels [97]. Dowell and Voss [99] performed flutter experiments on clamped rectangular, isotropic panels at Mach numbers of 1 to 5 and found good agreement with theoretical predictions provided the Mach number or length-towidth ratio of the panel was large. While this was an aeroelastic study the effect of thermally induced stresses was examined, and temperature differentials of the order of $5^{\circ} \mathrm{F}$ were found to significantly affect the panel frequencies and mode shapes 
[99]. Recently, an aerothermoelastic experiment was conducted in the AFRL RC19 wind tunnel to examine the response of an aircraft-like panel subject to Mach 2 flow and an impinging shock [100]. Using high-speed pressure sensitive paint and 3-D digital image correlation, the first simultaneous measurements of full-field dynamic pressure and structural response were obtained.

\subsubsection{Computational Analysis}

The challenges associated with experiments and the expense of flight tests motivates the need for computational analysis of aerothermoelastic effects in hypersonic flow. One approach is to use high-fidelity solvers for each of the disciplines (fluid, thermal, and structural). Ostoich et al. $[37,38]$ examined the coupled fluidthermal [37] and fluid-structural [38] response of metallic panels in high-speed flow through direct numerical simulation (DNS) of the compressible Navier-Stokes equations for a thermally perfect gas and finite element solvers for the heat conduction and structural dynamics. The difficulties associated with high-fidelity aerothermoelastic simulations are addressed in [37, 38]: (1) the fluid, structural, and thermal physics have disparate time scales and (2) the memory/computationalexpense required to resolve the length scales of turbulent motion limits DNS to relatively low Reynolds numbers. Note that the disparate time scales necessitate small time steps to capture the fluid behavior over long time records associated with the thermal response. This is an additional challenge that is inherent to the problem because of the thermal and material response. The path-dependent na- 
ture of structures in hypersonic flow is demonstrated through the change in flutter behavior with increasing surface temperature and state of the panel (i.e., flat or thermally buckled) reported in $[97,98]$. During flight the structure is continuously evolving due to aerodynamic heating, aerothermoelastic effects, and material evolution. Therefore, accurately predicting the structural response may require a coupled analysis over an entire trajectory or mission.

Previous work has employed alternatives to high-fidelity solvers for computationally tractable, long-time record aerothermoelastic simulation. One approach is to use low-fidelity, theoretical approximations to model one, or several, of the disciplines. Culler and McNamara assessed the aerothermoelastic behavior of panel structures in hypersonic flow by coupling unsteady theoretical and semi-empirical aerothermodynamic models to either theoretical [33] or finite element [101] thermal and structural models. The mutual coupling between the structural deformation and aerodynamic heating is found to reduce the flutter boundary and predict local regions where material temperature limits may be exceeded [33]. Additionally, the importance of the two-way interaction is related to the structural boundary condition through the resistance to in-plane expansion [101]. Other work has focused on the development of analytical models to assess how aspects of the physics that are not accounted for by low-fidelity theoretical approaches can alter the response of surface panels. Previous studies have examined the importance of temporally random fluctuating pressure loading, reminiscent of that in a turbulent boundary layer $[17,35]$. Inclusion of a spatially uniform pressure fluctuation was 
found to significantly reduce the flutter onset time [35]. Deshmukh et al. [17] found the spatially uniform assumption to be overly conservative through the use of random and semi-empirical models for the spatial variation in turbulent boundarylayer pressure fluctuation. Furthermore, the magnitude of the pressure fluctuation is found to increase with panel inclination and decrease with surface temperature. In a recent work, Lafontaine et al. [102] examined the effect of material plasticity on the structural response and life prediction of a panel subject to multiple loading cycles by incorporating a nonlinear strain hardening law into an aerothermoelastic framework. Plastic deformation was shown to increase the natural frequencies while simultaneously decreasing the frequency of the flutter behavior during the limit cycle oscillation of the panel.

Another means to account for the relevant physics while remaining computationally tractable is through developing reduced-order models (ROMs) from the high-fidelity (CFD/FEM) solvers. Common approaches for constructing CFDbased aerodynamic models [proper orthogonal decomposition (POD), Volterra series, and surrogates] seek to extract the relevant features of a system from a limited number of full-order flow solutions [31]. McNamara et al. [103] enhanced an inviscid aerodynamic pressure model, piston theory, using a steady-state CFD surrogate to account for the effective body shape of a double-wedge airfoil due to the boundary layer. This approach was later used to compute the long-timerecord, dynamic aerothermoelastic response of a 2-D skin panel [104] and a 3-D control surface [105]. In both [104, 105] kriging surrogates for the aerodynamic 
heating and pressure loads are developed from steady-state CFD solutions. For the heat flux surrogates surface temperature is parameterized using second- and third-order polynomials. Brouwer et al. [106] recently developed a local piston theory approach that accounts for externally generated flow discontinuities for the analysis of an oblique shock impinging on 2-D and 3-D flexible panels. The local flow properties are obtained from a steady-state CFD surrogate. Current uses of steady-state CFD kriging surrogates include the prediction of pressure on fullscale supersonic and hypersonic munitions accounting for varying angle-of-attack, side-slip angle, altitude range, wall temperature, and vehicle deformation [107]. Crowell et al. [108] developed a novel CFD-based surrogate approach to predict aerodynamic heat flux without a priori knowledge of the surface temperature profile. The surrogate was constructed from steady-state CFD flow solutions obtained at various surface deformations and uniform wall temperatures. Thermal gradients are accounted for using a correction term based on the analytical solution for the aerodynamic heating in a compressible, laminar boundary layer. Heat flux predictions were within 6.5 percent of the full-order CFD solutions for the cases examined [108]. The development of ROMs for solution of the thermal-structural problem has been addressed in prior work. Falkiewicz and Cesnik [109] found good agreement between a POD-based ROM and finite element analysis for solution of the transient heat transfer on a representative hypersonic vehicle control surface model. An extensive review of indirect methods for ROM construction to predict the response of geometrically nonlinear structures is provided in [110]. 
Indirect implies the ROMs are constructed using only standard output from FEM computations making these methods compatible with commercial FEM solvers. Perez et al. [36] developed thermoelastic ROMs for the geometrically nonlinear response heated structures. The ROM approach employed a modal-type expansion of both displacement and temperature in the undeformed, unheated configuration. The ROMs were validated for two structural models (isotropic beam and a functionally graded material panel) subject to a wide range of thermal and acoustic loads. The ROM predicted temperatures and displacements were in excellent agreement with finite element analysis [36].

The above discussion highlights progress made in the computational analysis of the aerothermoelastic response of structures in high-speed flow. The importance of coupling between the individual disciplines and of the oft neglected physics, such as turbulent boundary-layer pressure fluctuations and material plasticity, have been addressed. Efforts to provide computationally tractable, highfidelity aerothermoelastic simulation frameworks through the use of analyticaland reduced-order- modeling has been discussed. One aspect of hypersonic flight that has been neglected in the reviewed literature is boundary-layer transition, which can introduce significant uncertainty in the aerothermodynamic loads acting on a hypersonic vehicle. 


\subsubsection{Potential Impact of Transitional Boundary Layer on Struc- tural Response}

The transition to turbulence is associated with both global and local changes to the aerothermodynamic loads (heat flux, skin friction, fluctuating pressure) acting on an aircraft. Globally, each of the aforementioned aerothermodynamic loads increase as the flow transitions from laminar to turbulent. This is evident for skin friction through comparison of the laminar Blasius solution (pp. 235 of [58]) and the turbulent Schultz-Grunow formula [111] for a flat plate boundary layer [provided in Eq. (2.35)]. The heat flux follows a similar trend as it is related to the skin friction coefficient through the Reynolds analogy [58]. Additionally, numerous studies have shown the RMS of the fluctuating pressure rises significantly from laminar to turbulent flow $[27,28,112]$. Locally, throughout the transitional region these loads can exceed or overshoot the fully turbulent values. The overshoot in heat flux has been experimentally observed in the Calspan-University at Buffalo Research Center (CUBRC) large energy national shock (LENS I) hypervelocity reflected shock tunnel at Mach 6.5 and 7.2 [25]. Also, experiments on a flared cone geometry in the Boeing/AFOSR Mach 6 Quiet Tunnel (BAM6QT) at Purdue University have observed a hot-cold-hot phenomenon in which streaks of increased temperature occur on the cone followed by a decrease to near laminar levels [113]. DNS of Mach 6 flow over both a flat plate [26] and a flared cone [114, 115] indicate that the overshoot in heat flux and skin friction can result from first-mode oblique 
breakdown, which generates steady streamwise vortices that produce regions of high and low momentum and heat transfer.

As noted by Reshotko [116], the primary design consideration for vehicles operating above Mach 3 is reducing the aerodynamic heating in order to avoid the need of an active cooling system (i.e., additional weight). Therefore, accurate determination of the boundary-layer state is essential to the design of hypersonic vehicles. The importance of accurately predicting hypersonic boundary-layer transition is well established. In 1992, a Defense Science Board review of the National Aerospace Plane (NASP) program determined that increased confidence in the prediction of boundary-layer transition was of paramount importance to the NASP program [20]. In 2003, a Boeing Technical Fellowship Advisory Board on hypersonics identified boundary-layer transition prediction as one of the enabling technologies in the development of hypersonic systems [20]. Moreover, the geometry of the Hyper-X program X-43 vehicle was modified to ensure first-mode TollmienSchlichting waves, which can be controlled by wall cooling, were the dominant instability mechanism [116].

Despite the recognized importance of boundary-layer transition, limited research has accounted for transition in the aerothermoelastic response of structures on high-speed aircraft. To the best of the author's knowledge, beyond the present study only Lamorte and Friedmann [30] have included transition in an aerothermoelastic simulation. In [30] the effect of the transition location on the aerothermoelastic stability of a 3-D wing structure, resembling that of the Lockheed F-104 
Starfighter, is examined. Transition is incorporated by combining laminar and turbulent CFD predictions of the aerodynamic heat flux about a prescribed transition point. Lamorte and Friedmann [30] found that transitional heating alters the natural frequencies of the structure, producing responses that are not bounded by laminar and turbulent loading conditions. While [30] establishes the importance of accounting for transitional flow, the problem of transitional overshoot was not considered.

\subsection{Objectives of this Dissertation}

From the preceding literature review, it is evident that the stability and transition of hypersonic boundary layers are sensitive to changes in both the surface geometry and wall temperature. In addition, the response of structural components of hypersonic vehicles (e.g., wings or surface panels) are strongly dependent on the boundary-layer state. Despite this interdependence, limited work has examined: (1) the effect of large-scale surface deformations and temperature changes on boundary-layer stability and (2) the impact of transitional boundarylayer aerothermodynamic loading on structural response prediction. Thus, the objective of this dissertation is to examine the interaction between boundary-layer transition and the aerothermoelastic response of aerothermally compliant structures in high-speed flows. The specific objectives are:

(1) Assess the stability of boundary layers developing over large-scale, 2-D vary- 
ing surface topologies resembling the expected aerothermoelastic response of hypersonic vehicle surface panels.

(2) Develop and assess transitional boundary-layer aerothermodynamic load models.

(3) Examine the effects of transitional fluid loading on the thermostructural response of a representative hypersonic vehicle panel.

(4) Enhance an existing aerothermoelastic framework by incorporating a boundary layer transition prediction capability such that transition onset can vary in time as a function of the structural response.

(5) Compare panel responses obtained assuming the region of transitional fluid loading is uncoupled (fixed) or fully-coupled (time-varying) to the state of the structure.

(6) Identify aspects of the interaction that introduce uncertainty in the prediction of boundary-layer transition and the aerothermoelastic response of structures.

Note that objectives (1) to (3) examine the interaction between boundary-layer transition and structural response in an uncoupled sense. The coupled problem is considered in objectives (4) and (5).

The remainder of this dissertation is organized as follows: the computational tools used to assess the interaction between aerothermally compliant structures 
and boundary-layer transition are described in Chapter 2; the impact of structural deformation and temperature changes on boundary-layer stability is addressed in Chapter 3; variations in the aerothermoelastic response of a panel subject to laminar, turbulent, or prescribed transitional loading are presented in Chapter 4; a surrogate developed to predict boundary-layer transition is described in Chapter 5 along with panel responses obtained assuming the transition region is uncoupled or coupled to the state of the structure; and the principal conclusions and suggested future work are discussed in Chapter 6.

\subsection{Key Novel Contributions of this Dissertation}

The principal contributions of this dissertation are summarized as follows:

1. Identification of the potential for a series of 2-D panels buckled into the flow to delay boundary-layer transition. The stabilizing effect results from the deformation-induced pressure gradient, which alters the boundary-layer thickness, thereby changing the frequency of the most-unstable second-mode instability. Furthermore, a series of 2-D panels buckled out of the flowfield can promote transition through the repeated excitation of highly amplified, low-frequency disturbances.

2. Development of transitional boundary-layer heat flux and fluctuating pressure load models that account for the aerothermodynamic loads locally exceeding, or overshooting, the fully turbulent values during transition. 
3. Demonstration that the turbulent flow assumption does not provide a conservative estimate for determining the life of a surface panel, as transition with overshoot can result in earlier flutter onset and higher strain energy accumulation.

4. Development of a surrogate model, based on the linear PSE analysis of steadystate CFD solutions, for prediction of the boundary-layer transition onset location as a function of the deformation and wall temperature of a surface panel.

5. Development of an aerothermoelastic framework that accounts for the interaction between boundary-layer transition and the thermostructural response of a representative hypersonic vehicle surface panel.

6. Identification of the importance of transition onset location in relation to the temperature-dependent material properties, thermal bending moment, and structural boundary conditions.

7. Assessment of the interaction between boundary-layer transition and the state of the structure on the predicted aerothermoelastic response of a representative hypersonic vehicle surface panel. 


\section{Chapter 2}

\section{Computational Tools and Models}

Assessment of the interaction between aerothermally compliant structures and boundary-layer transition required the utilization, modification, and development of several computational tools and models. The individual software and models used in this work are described in detail in the subsequent sections.

\section{$2.1 \quad$ STABL}

The STABL software suite is a boundary-layer stability and transition analysis tool that incorporates grid generation, a 2-D/axisymmetric laminar flow solver based on the finite volume Data-Parallel-Line Relaxation (DPLR) method [117], mean flow analysis tools [118], and PSE-Chem [23, 117, 118] which uses linear stability theory (LST) and the linear Parabolized Stability Equations (LPSE) to assess the stability of the boundary layer. Transition is predicted using the semi-empirical

$e^{N}$ correlation method [23]. The approach requires an experimentally determined 
value of the integrated disturbance amplification rate $N$ at which transition occurs for a specific system. A number of experiments have found $N$ factors at transition ranging from 8 to 11 (sometimes even higher [119]) for smooth bodies in quiet flow and smaller values of approximately 5.5 in many cases for noisier environments such as non-quiet wind tunnels [23]. STABL has been extensively assessed in the analysis of many 2-D problems $[23,119,120]$ and is found to provide an adequate prediction of boundary-layer stability characteristics and transition through the $e^{N}$ method [120, 121].

\subsubsection{DPLR Mean Flow Solver}

The DPLR CFD solver provided in STABL solves the fully laminar Navier-Stokes equations, where the DPLR method allows the solver to be run in parallel. For this work, solution of the Navier-Stokes is obtained using a second-order accurate upwind MUSCL scheme for primitive variable reconstruction. The fluid is assumed to be calorically perfect air with a specific heat ratio of 1.4. A blended viscosity law, containing Sutherland and Blottner models, is used to compute the temperaturedependent viscosity. Thermal conductivity is computed using Eucken's relation [23]. The Prandtl number is approximately 0.74 for the range of freestream temperatures (216.7-231.2 K) considered.

For the geometries examined in this work, the computational domain resembles that shown in Fig. 2.1, where the lower surface is inclined to the required 
wedge half-angle and the upper surface is specified to capture the oblique shock. Each mesh is created using a FORTRAN script that makes use of STABL's mesh generation subroutines, a displacement map of the deformation, and the panel location along the wedge. Cells are clustered near the lower surface of the grid and exponentially stretched to the top surface to resolve the boundary layer while minimizing the cell count. Additionally, hyperbolic cell spacing is used to cluster cells near the leading edge of the wedge.

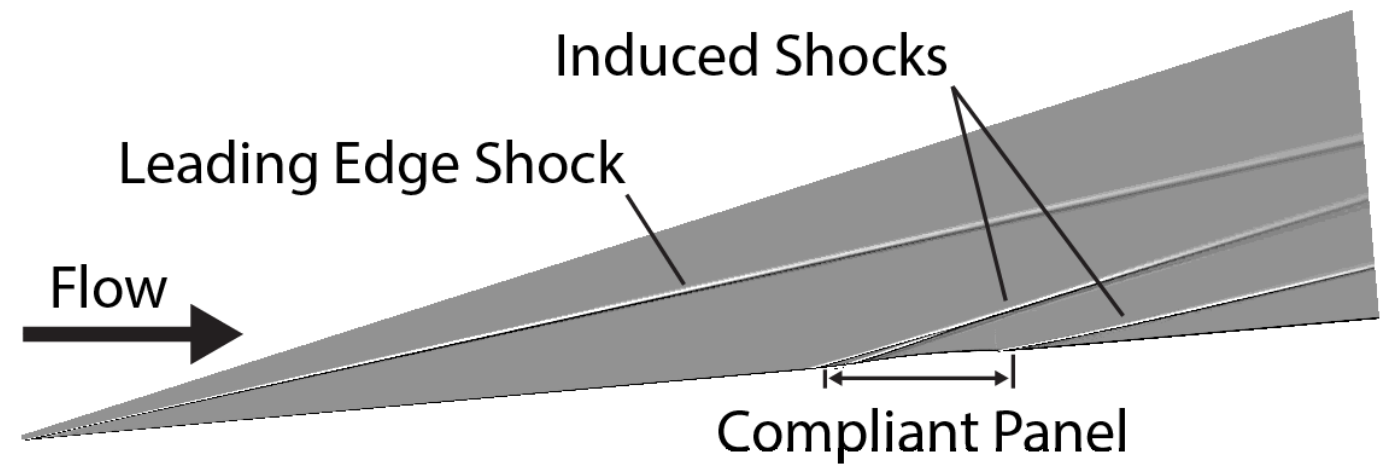

Figure 2.1: Shadowgraph highlighting flow modification due to surface deformation in hypersonic flow.

\subsubsection{PSE-Chem: Boundary-Layer Stability Analysis}

Both LST and LPSE analyses are derived by representing the instantaneous flow variables in the Navier-Stokes equations as the sum of a mean and disturbance component. Subtracting out the mean components that satisfy the Navier-Stokes equations and neglecting terms that are nonlinear with respect to the disturbances, results in the system of second-order PDE's in Eq. (2.1) where $\phi$ is the vector of 
disturbances quantities defined in Eq. (2.2).

$$
\begin{gathered}
\Gamma \frac{\partial \phi}{\partial t}+A \frac{\partial \phi}{\partial x}+B \frac{\partial \phi}{\partial y}+C \frac{\partial \phi}{\partial z}+D \phi+V_{x x} \frac{\partial^{2} \phi}{\partial x^{2}}+V_{y y} \frac{\partial^{2} \phi}{\partial y^{2}} \\
+V_{z z} \frac{\partial^{2} \phi}{\partial z^{2}}+V_{x y} \frac{\partial^{2} \phi}{\partial x \partial y}+V_{x z} \frac{\partial^{2} \phi}{\partial x \partial z}+V_{y z} \frac{\partial^{2} \phi}{\partial y \partial z}=0 \\
\phi=\left(\rho_{1}^{\prime}, \rho_{2}^{\prime}, \ldots, \rho_{n s}^{\prime}, u^{\prime}, v^{\prime}, w^{\prime}, T^{\prime}, T_{v}^{\prime}\right)^{T}
\end{gathered}
$$

The notation in Eq. (2.1) is consistent with that of Johnson [122], which follows the compressible LPSE formulation of Chang et al. [66]. The elements of the Jacobian matrices $\Gamma, A, B, C, D, V_{x x}, V_{y y}, V_{z z}, V_{x y}, V_{x z}$ and $V_{y z}$ in Eq. (2.1) only depend on the mean flow variables and their derivatives, obtained from the DPLR mean flow solver. The formulation of the disturbance equations in STABL can describe the stability of gas flow in thermal and chemical nonequilibrium, as shown by the multi-species densities " $n s^{\prime \prime}$ and the vibrational temperature $T_{v}^{\prime}$ in Eq. (2.2). This analysis employs the term "hypersonic" in a similar manner to [44] where the local Mach number is high enough for second-mode instabilities to be dominant and small enough to neglect real-gas effects. Fedorov and Khokhlov define this range as Mach 4 to 10 [44]. Therefore, for the results presented in this work, the gas is assumed to be calorically perfect air. This assumption provides a conservative estimate for transition prediction as real-gas effects have been shown to have a stabilizing influence on the boundary layer [123]. The equations are parabolized according to the method of Herbert [124], which assumes disturbances consist of a 
fast-oscillatory wave part and a slowly-varying shape function:

$$
\phi=\chi(x, y) e^{i(k z-\omega t)}
$$

Where $k$ is the real spanwise wavenumber and, for the spatial stability problem, $\omega$ is the real frequency. Specifying the spanwise wavenumber and frequency as real numbers corresponds to disturbance waves that are periodic in time and the spanwise direction. Substituting Eq. (2.3) into Eq. (2.1) reduces the linearized disturbance equations to:

$$
\tilde{\tilde{A}} \frac{\partial \phi}{\partial x}+\tilde{\tilde{B}} \frac{\partial \phi}{\partial y}+\tilde{D} \phi+V_{x x} \frac{\partial^{2} \phi}{\partial x^{2}}+V_{y y} \frac{\partial^{2} \phi}{\partial y^{2}}+V_{x y} \frac{\partial^{2} \phi}{\partial x \partial y}=0
$$

Where the matrices $\tilde{\tilde{A}}, \tilde{\tilde{B}}$, and $\tilde{D}$ are functions of the Jacobian matrices in Eq. (2.1), the spanwise wavenumber, and frequency as shown in Eqs. (2.5)-(2.7).

$$
\begin{gathered}
\tilde{\tilde{A}}=A+i k V_{x z} \\
\tilde{\tilde{B}}=B+i k V_{y z} \\
\tilde{D}=-i \omega \Gamma+i k C+D-k^{2} V_{z z}
\end{gathered}
$$

To account for body-fitted curvilinear grids, Eq. (2.4) is transformed into the computational domain in terms of the body-parallel and normal variables $\xi$ and $\eta$ in 
Eq. (2.8). Definitions for $\tilde{A}, \tilde{B}, \tilde{D}, \hat{V}_{\xi \xi}, \hat{V}_{\eta \eta}$, and $\hat{V}_{\xi \eta}$ are provided in [122].

$$
\tilde{A} \frac{\partial \phi}{\partial \xi}+\tilde{B} \frac{\partial \phi}{\partial \eta}+\tilde{D} \phi+\hat{V}_{\xi \xi} \frac{\partial^{2} \phi}{\partial \xi^{2}}+\hat{V}_{\eta \eta} \frac{\partial^{2} \phi}{\partial \eta^{2}}+\hat{V}_{\xi \eta} \frac{\partial^{2} \phi}{\partial \xi \partial \eta}=0
$$

The slowly-varying shape function $\chi$ is further decomposed into the shape function $\psi$ and an amplitude function $\Lambda$, where the derivative of $\Lambda$ with respect to $\xi$ is proportional to the complex wavenumber parallel to the body in the computational coordinates $\alpha(\xi)$.

$$
\begin{aligned}
& \chi=\psi(\xi, \eta) \Lambda(\xi) \\
& \Lambda(\xi)=e^{i \int \alpha(\xi) d \xi}
\end{aligned}
$$

Substituting the relation for $\chi$ in Eq. (2.9) into Eq. (2.8) provides the disturbance equations in computational coordinates as a function of the complex wave number $\alpha$ and the shape function $\psi$, from which both LST and the LPSE are derived.

$$
\begin{gathered}
\hat{D} \psi+\hat{A} \frac{\partial \psi}{\partial \xi}+\hat{B} \frac{\partial \psi}{\partial \eta}+\hat{V}_{\xi \xi} \frac{\partial^{2} \psi}{\partial \xi^{2}}+\hat{V}_{\eta \eta} \frac{\partial^{2} \psi}{\partial \eta^{2}}+\hat{V}_{\xi \eta} \frac{\partial^{2} \psi}{\partial \xi \partial \eta}=0 \\
\hat{D}=\tilde{D}+i \alpha \tilde{A}+\hat{V}_{\xi \xi}\left(-\alpha^{2}+i \frac{\partial \alpha}{\partial \xi}\right) \\
\hat{A}=\tilde{A}+2 i \alpha \hat{V}_{\xi \xi} \\
\hat{B}=\tilde{B}+i \alpha \hat{V}_{\xi \eta}
\end{gathered}
$$




\section{Linear Stability Theory}

To derive the LST equations a quasi-parallel flow assumption is made such that streamwise gradients of flow quantities are neglected. This assumption removes the dependence of $\psi$ and $\alpha$ on the body parallel coordinate $\xi$ and therefore $\chi$ can be expressed as:

$$
\chi=\psi(\eta) e^{i \alpha}
$$

The quasi-parallel flow assumption allows all derivatives with respect to $\xi$ in Eq. (2.11) to be neglected, resulting in the relation in Eq. (2.16).

$$
\hat{D} \psi+\hat{B} \frac{\partial \psi}{\partial \eta}+\hat{V}_{\eta \eta} \frac{\partial^{2} \psi}{\partial \eta^{2}}=0
$$

The expression in Eq. (2.16) is a second order ODE, generalized eigenvalue problem, which is solved for the complex wave number $\alpha$ and shape function $\psi$ provided values of the spanwise wavenumber $k$ and frequency $\omega$. Note that when the imaginary component of $\alpha$ is negative, the amplitude of $\chi$ grows exponentially along the body, corresponding to an unstable disturbance.

\section{Linear Parabolized Stability Equations}

The LPSE allow for streamwise variation in the shape function $\psi$ and are derived from Eq. (2.11) by assuming $\frac{\partial \psi}{\partial \xi}$ is small, thereby, allowing the higher derivative terms in $\xi$ to be neglected. The resulting expression in Eq. (2.17) is an initial bound- 
ary value problem for the disturbance shape function and wavenumber that is solved in a marching procedure. The initial values for $\psi$ and $\alpha$ used to start the marching procedure are obtained through solution of the LST equations. While both LST and the LPSE predict boundary-layer stability, the LPSE provide a significant advantage over LST as they account for the history of disturbances and the streamwise variation of the basic flow [125].

$$
\hat{D} \psi+\hat{A} \frac{\partial \psi}{\partial \xi}+\hat{B} \frac{\partial \psi}{\partial \eta}+\hat{V}_{\eta \eta} \frac{\partial^{2} \psi}{\partial \eta^{2}}=0
$$

\subsubsection{Transition Prediction}

STABL provides a means to predict boundary-layer transition through the semiempirical $e^{N}$ correlation method [23], where $N$ represents the spatial amplification of a constant frequency disturbance. The $N$ factor in Eq. (2.18) is defined as the spatially integrated disturbance growth rate, where $S$ is the distance along the surface, $S_{o}$ is the first neutral point of a fixed-frequency $\omega$ disturbance, and $E$ is the disturbance kinetic energy.

$$
N(\omega, S)=-\int_{S_{o}}^{S}\left[\alpha_{i}(S, \omega)+\frac{1}{2 E} \frac{d E}{d S}\right] d S
$$

The approach is semi-empirical as it requires an experimentally determined value of $N$ at which transition occurs for a specific system. A number of experimental studies have found $N$ factors at transition ranging from 8 to 11 (sometimes 
even higher [119]) for smooth bodies in quiet flow and smaller values of approximately 5.5 in many cases for noisier environments such as non-quiet wind tunnels [23]. In this work, $N$ factors are computed for a broad range a frequencies. The transition onset location is defined based on the maximum $N$ factor envelope for the frequency range considered.

\subsubsection{STABL Results}

As previously stated, the LPSE analysis in STABL is initiated through solution of the LST equations. For this reason, both approaches are considered in this work, where LST is used to qualitatively assess the effects of surface deformation and temperature variation on boundary-layer stability and LPSE is used to predict the expected shift in the transition location. To illustrate how each approach is used, an example is provided in Fig. 2.2 for Mach 5 flow over a smooth wedge with a $5^{\circ}$ half angle.

Results from the LST analysis are shown in Fig. 2.2.a where the contoured value is the negative, imaginary component of the complex wave number $\left(-\alpha_{i}\right)$ for a fixed-frequency disturbance. Expanding the disturbance shape function expression in Eq. (2.15) in terms of the real and imaginary components of $\alpha$ reveals that contour levels greater than zero correspond to unstable disturbance growth while contour level zero and below contains the neutral and stable disturbances. The highly contoured band in Fig. 2.2.a represents the unstable frequency range in the 
spatial domain. This figure conveniently highlights two factors that can lead to transition: (1) the disturbance amplification rate (shown by the contour level), and (2) the total distance along the body over which a constant frequency disturbance is unstable (shown by the horizontal width of the highly contoured band).

In hypersonic flow, the most unstable disturbances tend to be Mack waves (i.e., trapped acoustic waves) which propagate between the wall and the sonic line [67]. Thus, the disturbance frequency depends on the distance between the wall and the sonic line. Boundary-layer thickness increases with distance from the leading edge in the absence of a streamwise pressure gradient, as is the case for the results in Fig. 2.2. Therefore, as the boundary-layer thickens, the sonic line within the boundary layer becomes further away from the wall and lower frequency disturbances become the most unstable. This relationship between boundary-layer thickness and disturbance frequency explains the unstable band of disturbances in Fig. 2.2.a decreasing as $1 / \sqrt{S}$. As a result, high-frequency disturbances are strongly amplified for short distances near the leading edge and lower-frequencies are weaklyamplified over large distances towards the trailing edge. While the highest amplification rates occur near the leading edge, the frequencies are only unstable for a short distance along the body. Transition occurs when there is both spatial amplification of disturbances and a sufficient distance for the unstable growth.

Disturbance growth rates, obtained through solution of the LPSE, are used to compute $N$ factors for transition prediction. $N$ factor curves are shown in Fig. 2.2.b, where each colored line corresponds to a specific disturbance frequency. 


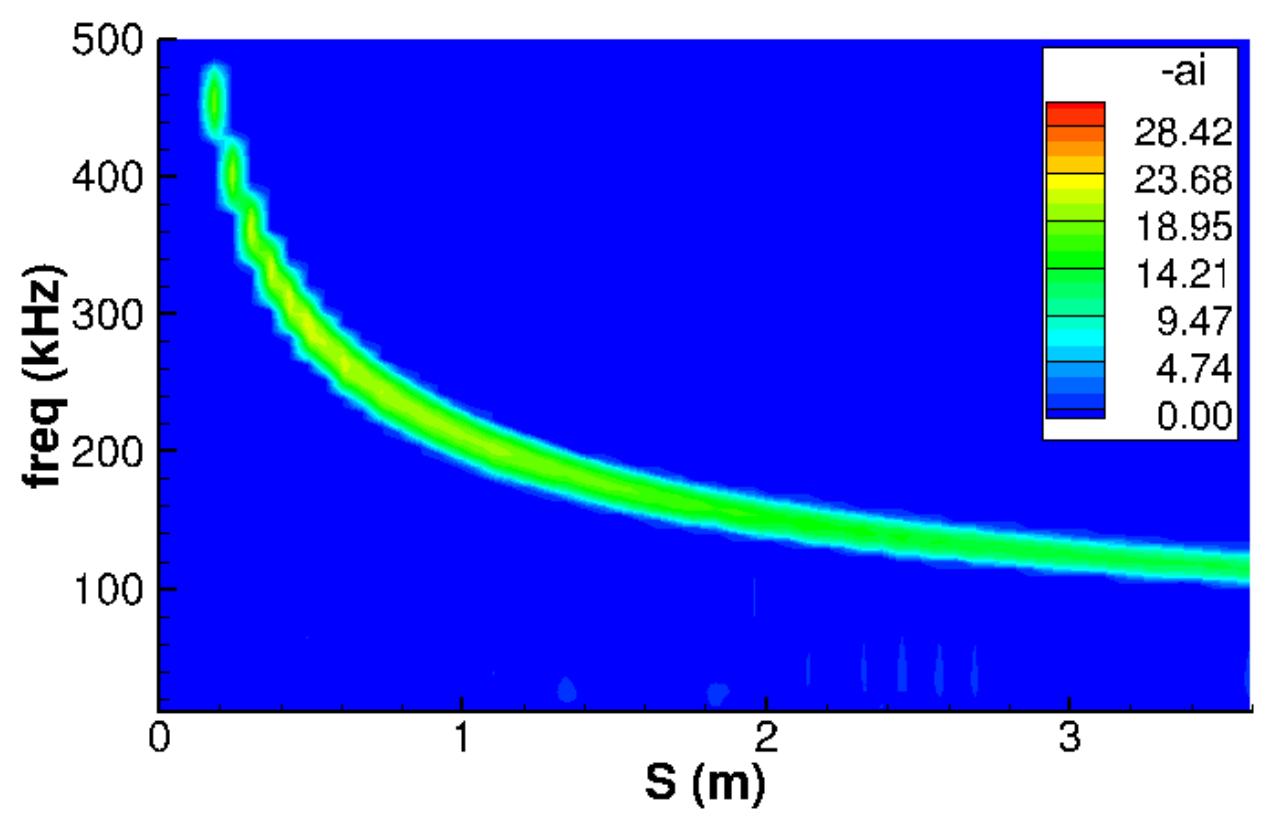

(a) Stability diagram from LST.

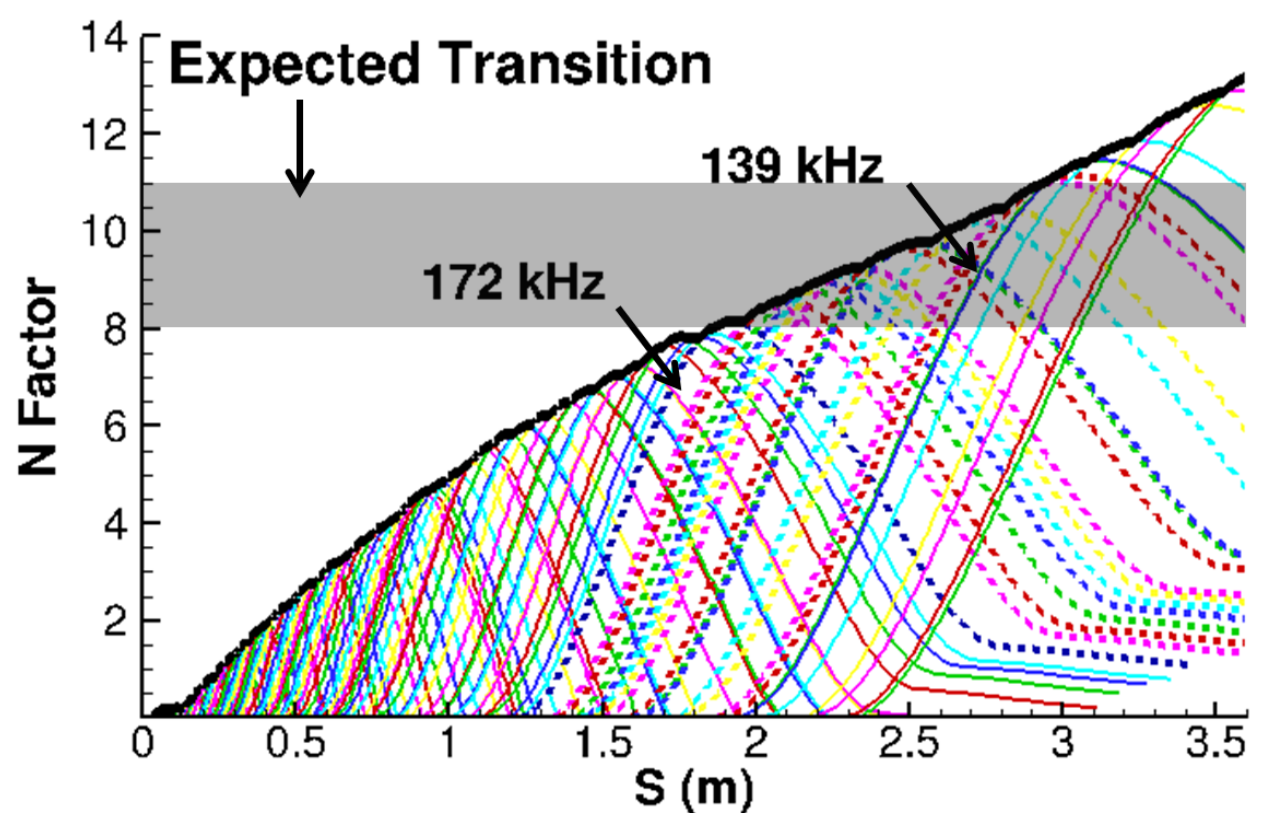

(b) LPSE computed $N$ factors for a broad range of disturbance frequencies.

Figure 2.2: LST and LPSE results for Mach 5 flow past a $5^{\circ}$ half-angle smooth wedge in Mach 5 flow. 
Transition onset is predicted based off the maximum $N$ factor envelope (solid black line in Fig. 2.2.b). Assuming a transitional $N$ factor of 8-11, as shaded in Fig. 2.2.b, transition onset would occur 1.8 to $2.9 \mathrm{~m}$ from the leading edge. The dashed lines in Fig. 2.2.b highlight that transition would result from disturbance amplification within the frequency range of $139-172 \mathrm{kHz}$.

\subsection{Surrogate Modeling}

As eloquently stated in [126], surrogate models are essentially "educated guesses as to what an engineering function might look like, based on a few points in space where we can afford to measure the function values." Surrogate models are advantageous as they offer a low-cost alternative to the original engineering function. For this reason, the engineering function that is being replaced by the surrogate is typically a computationally-expensive, deterministic simulation code (e.g., RANSbased CFD [108]). The construction of a surrogate model involves evaluating an engineering function, or simulation code, for a limited number of discrete input conditions. Using the input-output relations at the evaluation sites, a surrogate function is determined that best approximates the behavior of the original engineering function.

Depending on the approach, the surrogate function can take many forms. One approach is the response-surface technique, in which the input-output data at the evaluations sites are fit to a simple function, typically a polynomial, using least- 
squares regression [127]. As a result of the least-squares regression, responsesurface models introduce error even at the evaluation sites. The approach that is used in this work is an interpolation method known as kriging which is an advanced response surface technique.

The kriging approach originated in the field of geostatistics [128, 129] for mining applications and was later adopted as a means to reduce the expense of computational experiments [130]. Kriging treats the deterministic evaluation of an engineering function as a realization of a stochastic process [130] and approximates the engineering function as:

$$
Y(x)=f(x)+Z(x)
$$

where $f(x)$ is a regression model and $Z(x)$ is a random process with mean zero, variance $\sigma^{2}$, and nonzero covariance given by Eq. (2.20):

$$
\operatorname{Cov}\left[Z\left(x^{i}\right), Z\left(x^{j}\right)\right]=\sigma^{2} R\left(\theta, x^{i}, x^{j}\right)
$$

where $R\left(\theta, x^{i}, x^{j}\right)$ is a correlation model with parameters $\theta$. The regression model provides a global approximation of the system while $Z(x)$ captures local deviations from the regression [127]. By treating the response as a realization of a stochastic process, kriging provides a statistical basis for computing an efficient predictor of the response at untried inputs [130]. 
An example of a kriging surrogate model that approximates an engineering function with two inputs and one output is provided in Fig. 2.3. First, a set of function evaluations are generated to train the surrogate (Fig. 2.3.a). The training data is fit with a regression model and a stochastic process $Z(x)$ that captures the local deviations of the training data from the regression model. This results in a surrogate that accurately predicts the response at each training point (Fig. 2.3.b). The correlation model controls the shape of the "deviations" from the regression model and the $\theta$ parameters affect how far the influence of a training point extends. Further detail on the generation and usage of kriging models is provided in [126].

For the results presented in Chapter 5, a kriging surrogate model is generated in order to reduce the online computational expense associated with STABL-based transition prediction within an aerothermoelastic response simulation. The kriging surrogates are generated with the Design and Analysis of Computer Experiments (DACE) MATLAB ${ }^{\circledR}$ toolbox [131]. The regression model is specified as a second degree polynomial and a Gaussian correlation is prescribed for the random process.

\subsection{Aerothermoelastic Model}

The aerothermoelastic model, depicted in Fig. 2.4, has three primary components: 1) aerothermodynamic loads, 2) structural dynamics, and 3) heat transfer. The aerothermodynamics drive the thermostructural response through the application 


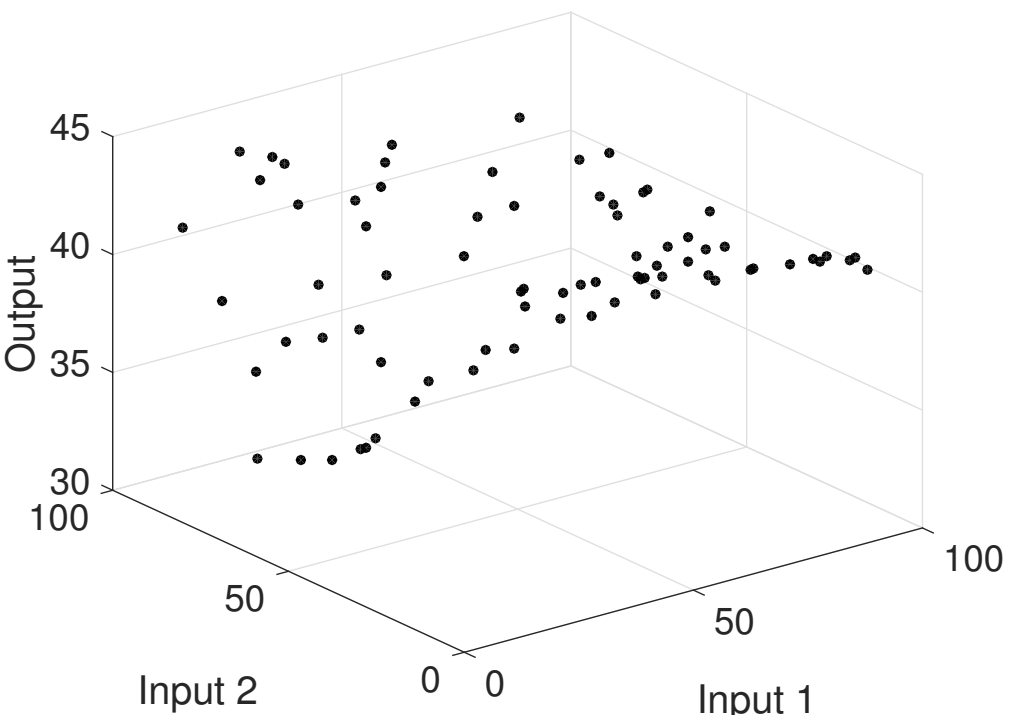

(a) Engineering function evaluation points.

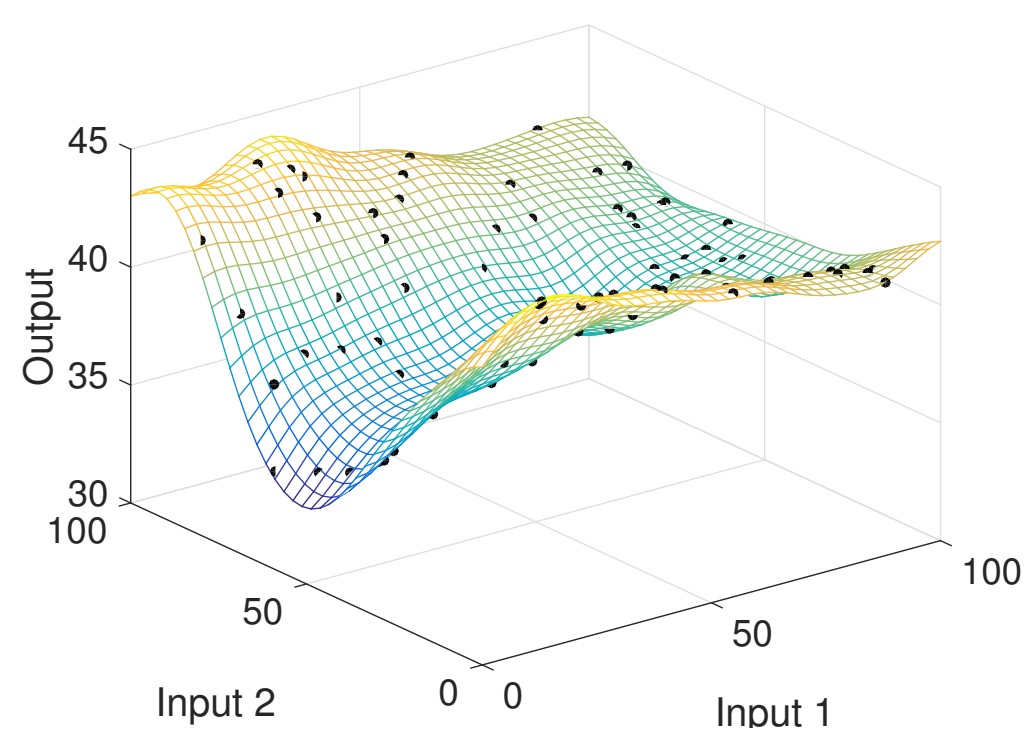

(b) Kriging model.

Figure 2.3: Kriging surrogate example. 
of a pressure load (composed as the summation of mean and fluctuating components) and a surface heat flux. The mean flow pressure is modeled using thirdorder piston theory $[33,132,133]$ which accounts for changes in the mean pressure due to structural deformations. The fluctuating pressure load is computed using the turbulent boundary-layer fluctuating pressure model discussed in Section 2.3.2.2. The surface heat flux can account for both aerodynamic heating, modeled using Eckert's reference enthalpy method [134], and radiation between the structure and the freestream, modeled using:

$$
Q_{\text {rad }}(x)=\sigma \epsilon\left(T_{w}^{4}-T_{\infty}^{4}\right)
$$

where $\sigma$ is the Stefan-Boltzmann constant and $\epsilon$ is the panel emissivity. The fluctuating pressure and heat flux are dependent on the boundary-layer edge properties, which are obtained from the mean flow pressure in conjunction with isentropic flow relations [33]. This introduces an implicit coupling with the structural response. A recent comparison with RANS based aerothermodynamics indicates that the analytical models used here adequately capture the characteristic aerothermoelastic response [135]. The models used to obtain the structural temperature and dynamics are discussed in Sections 2.3.4-2.3.5.

The aerothermal and aeroelastic solvers are linked using a loosely coupled partitioned approach. This scheme is advantageous in terms of computational efficiency as the individual solvers can use different time steps and information is ex- 


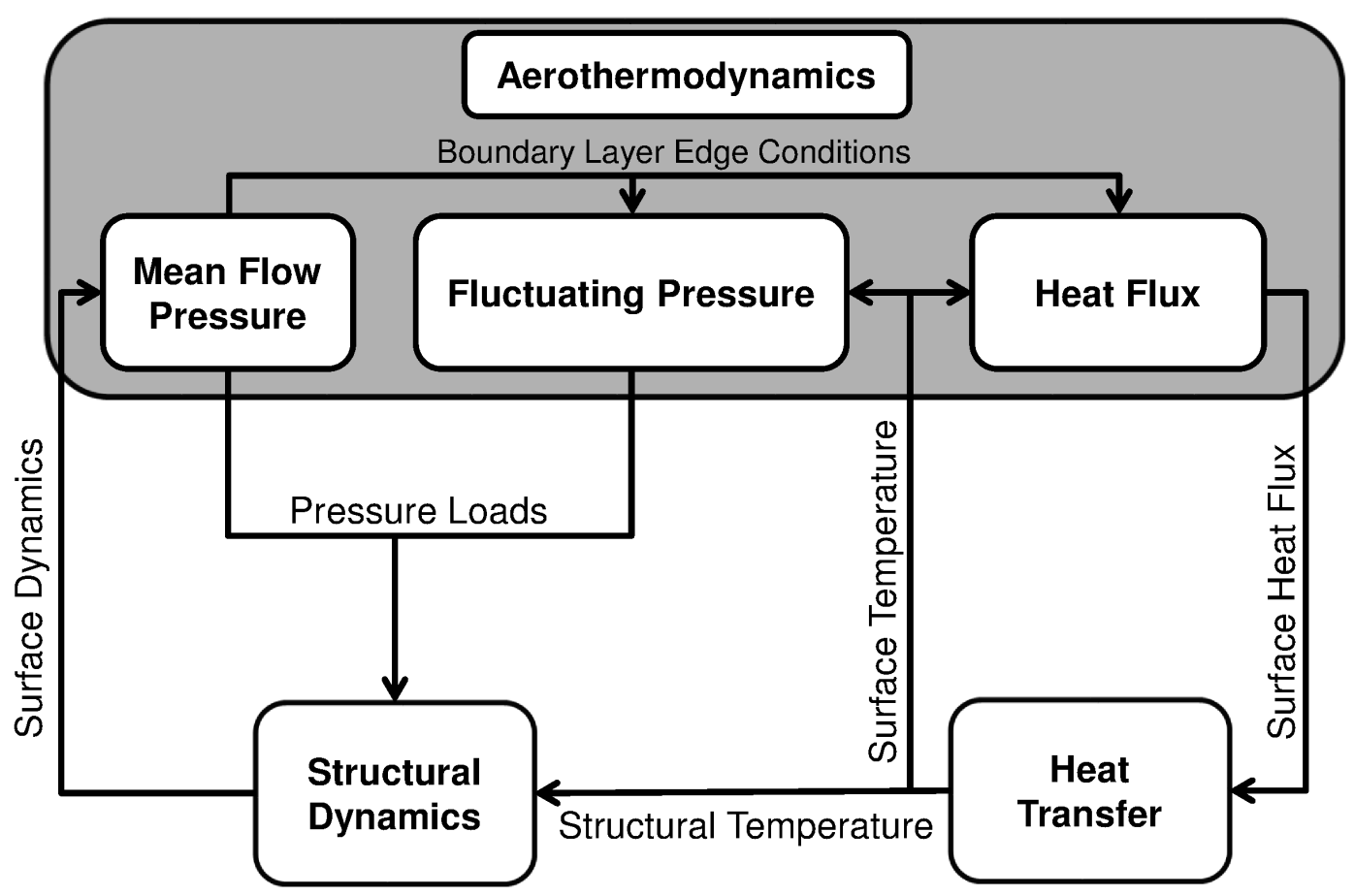

Figure 2.4: Aerothermoelastic model.

changed between the solvers only once per time step [136]. In-depth descriptions of the aerothermoelastic model formulation are provided in $[33,35]$. Further information regarding the coupling procedure and numerical schemes implemented in the aerothermoelastic model is given in [136]. For the results in Chapter 4 and Chapter 5 the aeroelastic time step is $12.5 \mu$ s while the aerothermal solution is updated at a coarser time step of $125 \mu$ s. These time steps were determined through a convergence study of the post-instability, limit-cycle response of the panel when subject to transitional boundary-layer aerothermodynamic loading. Power spectral densities, corresponding to the post-flutter limit-cycle displacement at the $3 / 4$ chord location, are provided in Fig. 2.5 for different aeroelastic (Fig. 2.5.a) and aerothermal (Fig. 2.5.b) time steps. A comparison of the PSDs in Fig. 2.5.a indi- 
cates that the dominant frequency peaks are converged by the aeroelastic time step of $25.0 \mu \mathrm{s}$. For a fixed aeroelastic step ( $\Delta t_{A E}=12.5 \mu \mathrm{s}$; Fig. 2.5.b), the dominant peaks are converged by a time-step ratio of $10\left(\Delta t_{A T}=125 \mu \mathrm{s}\right)$. Convergence of the dominant limit-cycle frequencies was achieved with 13 structural modes using the aeroelastic and aerothermal time steps of $12.5 \mu \mathrm{s}$ and $125 \mu \mathrm{s}$, respectively. However, 25 structural modes are retained for the panel responses in this work as the computational penalty of the additional modes is negligible. The time steps and number of structural mode shapes used for the aerothermoelastic analysis in this work are listed in Table 2.1.

Table 2.1: Numerical parameters of the aerothermoelastic solution.

\begin{tabular}{lc}
\hline \hline Parameter & Value \\
\hline Number of structural modes & 25 \\
Aeroelastic time step, $\mu$ s & 12.5 \\
Aerothermal time step, $\mu \mathrm{s}$ & 125 \\
\hline \hline
\end{tabular}

\subsubsection{Enhancements to the Aerothermoelastic Model}

The aerothermoelastic model in Fig. 2.4 was modified to account for transitional boundary-layer aerothermodynamic loads. The enhanced framework, depicted in Fig. 2.6, incorporates transitional boundary-layer effects into the heat flux and fluctuating pressure loads. The transition onset location and length of the transition region are prescribed. Additionally, the transition onset location can vary throughout the aerothermoelastic simulation as a function of the structural re- 


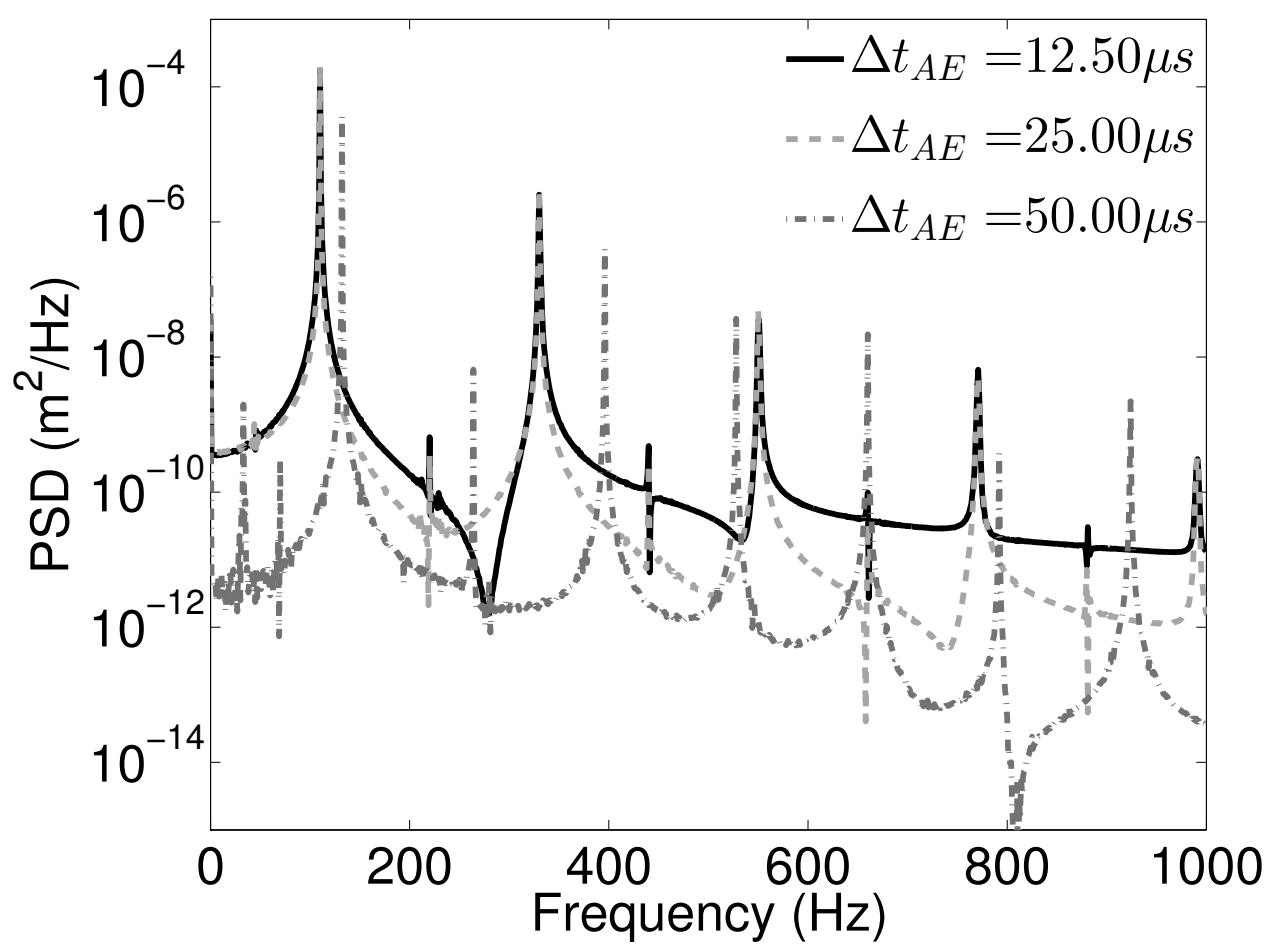

(a) Aeroelastic time step $\left(\Delta t_{A T}=10 \Delta t_{A E}\right)$.

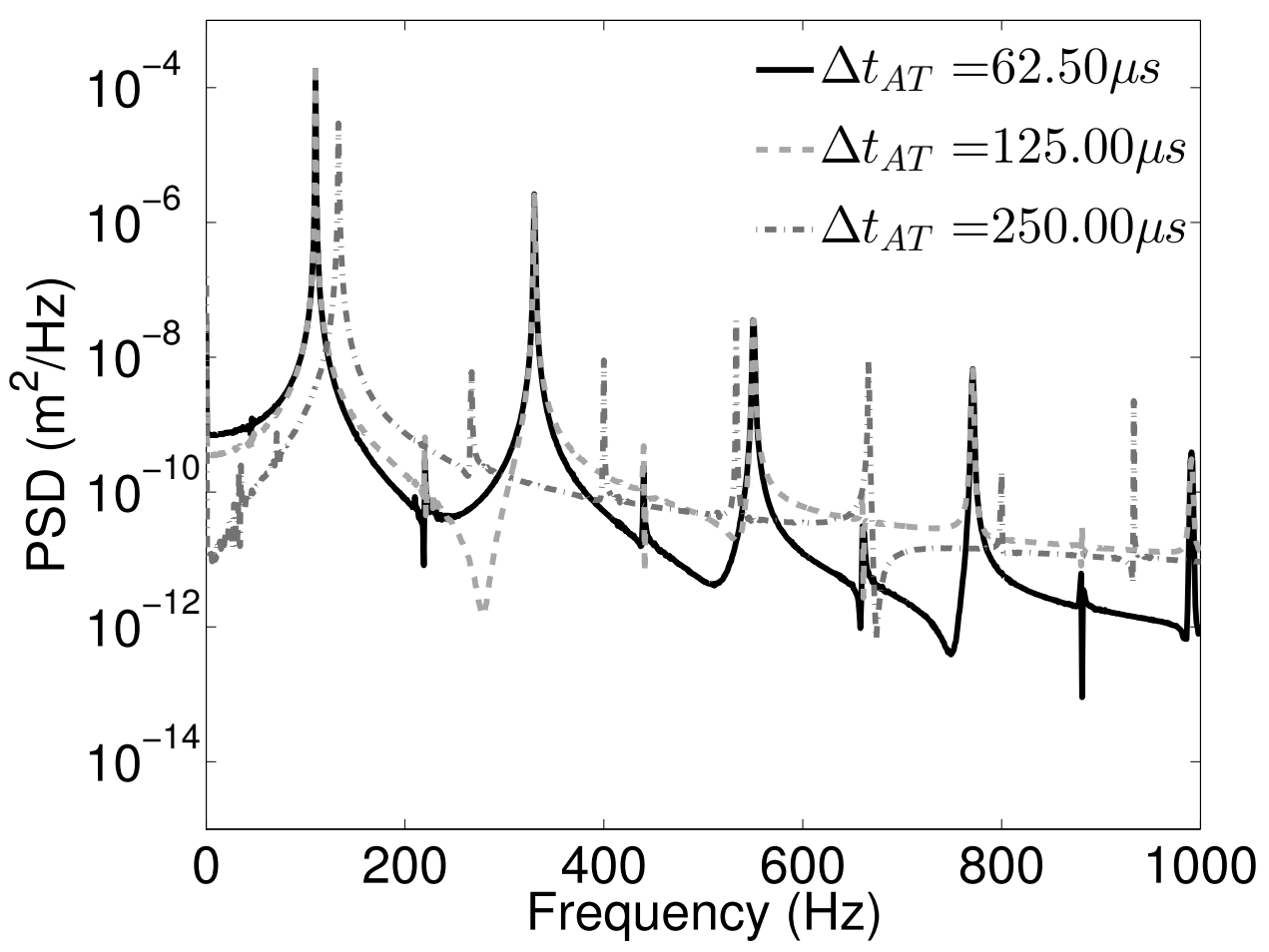

(b) Aerothermal time step $\left(\Delta t_{A E}=12.5 \mu \mathrm{s}\right)$.

Figure 2.5: Power spectral density at 3/4-chord length (transitional overshoot $x_{t}=$ $\left.0.3 \mathrm{~m}, x_{t e}=0.5 \mathrm{~m}\right)$. 
sponse. Boundary-layer transition prediction in the aerothermoelastic model is accomplished using either STABL or a surrogate-based approach, which is discussed in Section 5.2. In either case, transition prediction is based on the maximum $N$ factor envelope for a range of disturbance frequencies. The transition onset location is defined as the most-forward location where the maximum $N$ factor curve achieves a specified, transitional $N$ factor. As transition prediction is dependent on the structural response (i.e., wall temperature and deformation) the onset location corresponding to a constant transitional $N$ factor will vary in time, potentially moving downstream. Rearward movement of the onset location indicates that a region of turbulent flow has relaminarized due to the structural response. However, previous work indicates that the transitional $N$ factor for a relaminarized boundary layer is significantly smaller than that for a fully laminar boundary layer [137]. Due to uncertainty in the transitional $N$ factor for relaminarization, transition prediction in the aerothermoelastic model is specified such that the onset location does not move downstream (i.e., relaminarization is neglected). Therefore, during an aerothermoelastic simulation the transition onset location will always coincide with the most upstream prediction. In Chapter 4, the aerothermoelastic model in Fig. 2.6 is used to assess the impact of a fixed region of transitional fluid loading on the panel response, where the transition onset and length are prescribed prior to the simulation. For the results in Chapter 5, the transition onset location varies in time as a function of the structural response. 


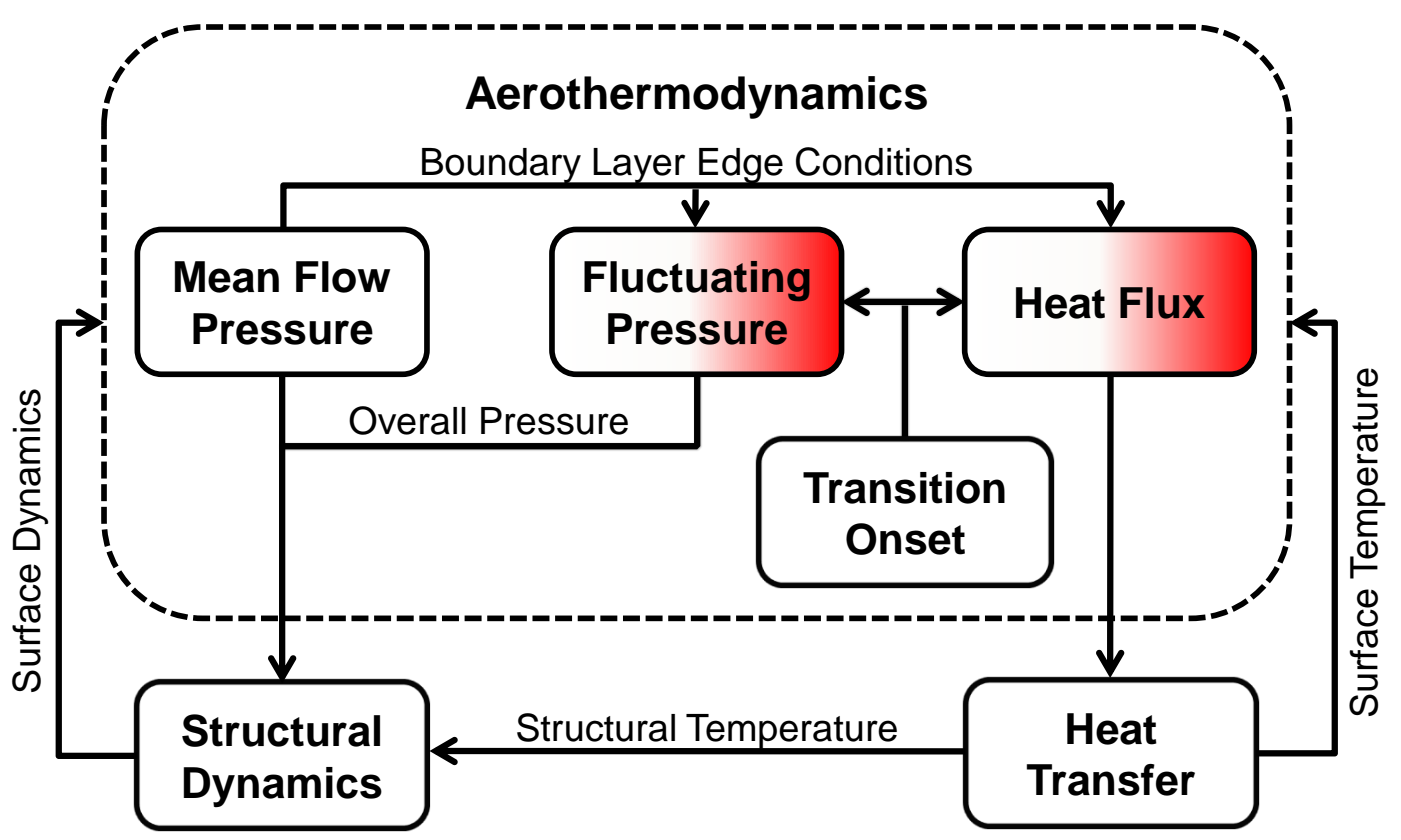

Figure 2.6: Enhanced aerothermoelastic model.

\subsubsection{Aerothermodynamic Loads}

\subsubsection{Piston Theory}

Classical piston theory provides a pointwise relationship between the local surface pressure and surface motion (i.e., inclination and velocity). The method is applicable to high Mach number flows with thin shock layers. Following the work of Lighthill [132], classical piston theory is derived from the isentropic simple wave expression in Eq. (2.22), where $v_{n}$ is defined in Eq. (2.23). Note that $v_{n}$ represents the wall-normal velocity component of a slab of fluid convecting downstream at $U_{\infty}$ and takes into account the motion of a surface $Z(x, y, t)$.

$$
\frac{P}{P_{\infty}}=\left(1+\frac{\gamma-1}{2} \frac{v_{n}}{a_{\infty}}\right)^{\frac{2 \gamma}{(\gamma-1)}}
$$




$$
v_{n}=\frac{\partial Z(x, y, t)}{\partial t}+U_{\infty}\left\{\frac{\partial Z(x, y, t)}{\partial x}\right\}
$$

In this work, the third-order binomial expansion of the simple wave expression in Eq. (2.24), termed third-order piston theory, is used to compute surface pressure. Lighthill recommended the use of third-order piston theory as it predicts pressures within $6 \%$ of the simple wave and shock expansion theories [132].

$$
\frac{P}{P_{\infty}}=1+\gamma\left\{\frac{v_{n}}{a_{\infty}}+\frac{(\gamma+1)}{4}\left(\frac{v_{n}}{a_{\infty}}\right)^{2}+\frac{(\gamma+1)}{12}\left(\frac{v_{n}}{a_{\infty}}\right)^{3}\right\}
$$

\subsubsection{Fluctuating Pressure Model}

The fluctuating pressure load model is a modified version of the semi-empirical model developed by Deshmukh et al. [17] for turbulent boundary layers. The unsteady pressure due to the turbulent boundary layer is expressed as shown in Eq. (2.25), where $f(x, t)$ and $\Theta(x, t)$ represent the magnitude and phase angle of the pressure load.

$$
p=f(x, t) e^{i[\omega t+\Theta(x, t)]}
$$

As denoted in Eq. (2.26), the phase angle is decomposed into separate temporal $\tau$ and spatial $\psi$ phase angles where the spatial variation is assumed relative to the leading edge of the panel. The temporal phase angles account for the phase lag between disturbances of different frequency at the same spatial location. Conversely, the spatial phase angles account for the phase lag between disturbances of 
the same frequency at different spatial locations.

$$
\Theta(x, t)=\tau(t)+\psi(x)
$$

Phase angle is a key parameter for structural response prediction [17], as it greatly impacts the ability of the flow to transmit energy to the structure. In this work, the phase angles $(\tau$ and $\psi$ ) are assumed to vary randomly with $x$ and $t$. A recent study by Deshmukh et al. [17] indicates that a random representation of the phase angles is a reasonable assumption for fully turbulent attached flows, and a logical choice in the absence of empirical correlations for transitional flows. However, the presence of coherence can have a significant impact on the structural response, and warrants future study for transitional flows [138]. The amplitude $f(x, t)$ is described as the combination of a root mean square (RMS) value, corresponding to the magnitude, and a power spectral density (PSD), corresponding to the frequency content $[18,139,140]$.

The RMS pressure is computed using Eq. (2.27), which is a modified version of Laganelli's relation for attached flow $[18,139,140]$. The first modification involves increasing the incompressible fluctuating pressure intensity from 0.006 to 0.009 , as recommended by Bull [141] and Beresh et al. [142]. The second modification introduces an $R e_{\theta}^{-0.1}$ dependence into the RMS pressure calculation, which Beresh 
et al. [142] found to exist for Mach numbers between 2 to 3 .

$$
\frac{\tilde{p}}{q_{e}}=0.009 F_{c}^{\lambda(1+b)}\left(\frac{R e_{\theta}}{\left.R e_{\theta}\right|_{\bar{x}^{*}}}\right)^{-0.1}
$$

In order to examine this modified formulation, Eq. (2.27) is used to reproduce experimental RMS pressures obtained in the Sandia Trisonic Wind Tunnel [142]. A comparison is provided in Fig. 2.7 at two Mach numbers, where the experimental data are extracted from figure 14.a of [142]. In Fig. 2.7, curves labeled "no $R e_{\theta}$ " neglect the Reynolds number dependence in Eq. (2.27) and are analogous to Laganelli's model (equation 14 of [18]) with an incompressible pressure intensity of 0.009. As shown in Fig. 2.7, the $n o-R e_{\theta}$ model overpredicts the experimental data, with improved agreement as the Mach number increases. This error is inherit to Laganelli's model, resulting from its treatment of compressibility effects. The solid curves in Fig. 2.7 correspond to the momentum thickness Reynolds number $\left.R e_{\theta}\right|_{\bar{x}^{*}}$ specified at the most upstream, experimental measurement location. The improved agreement, over the no- $R e_{\theta}$ curve, illustrates the importance of the $R e_{\theta}$ parameter in modeling the fluctuating pressure. Note that the value of $\left.R e_{\theta}\right|_{\bar{x}^{*}}$ in Eq. (2.27) can be specified to provide a better fit to the experimental data in Fig. 2.7. Here, $\left.R e_{\theta}\right|_{\bar{x}^{*}}$ is specified at the first experimental measurement location to introduce the Reynolds dependence over the location of interest assuming no a priori knowledge of experimental results. As no experimental RMS pressure is available for the configurations examined in this work, the Reynolds dependence is speci- 
fied to begin at the leading edge of the panel. The results in Fig. 2.7 demonstrate that by specifying $\left.R e_{\theta}\right|_{\bar{x}^{*}}$ in this manner the predicted RMS values are conservative and provide improved accuracy over Laganelli's original model.

The PSD is computed using Eq. (2.28), where the parameter $F_{c}^{-2 \lambda}$ represents the compressibility and heat transfer effects.

$$
\frac{\phi(x, \omega) u_{e}}{q_{e}^{2} \delta_{1}}=\frac{\left(\tilde{p} / q_{e}\right)^{2} F_{c}^{-2 \lambda}(2 / \pi)}{1+\left(F_{c}^{-2 \lambda} \omega \delta_{1} / u_{e}\right)^{2}}
$$

The fluctuating pressure load, acting on the panel, is obtained by converting the frequency domain PSD values and phase angles to a time domain signal using the analytical function provided in Eq. (2.29):

$$
p(x, t)=\int_{0}^{\omega_{\max }} \sqrt{2 \phi^{*}(x, \omega) \Delta \omega} \cos [\omega t+\Theta(x, \omega)] d \omega
$$

This function is the real component of a one-sided inverse Fourier transform, where the upper limit of integration $\omega_{\max }$ corresponds to the largest frequency expected to impact the structure. As energy is removed due to the frequency truncation, the pressure signal must be computed using a scaled PSD [Eq. (2.30)] in order to reproduce a fluctuating pressure that matches the input RMS values.

$$
\phi^{*}(x, \omega)=\frac{\tilde{p}^{2}}{\int_{0}^{\omega_{\max }} \phi(x, \omega) d \omega} \phi(x, \omega)
$$




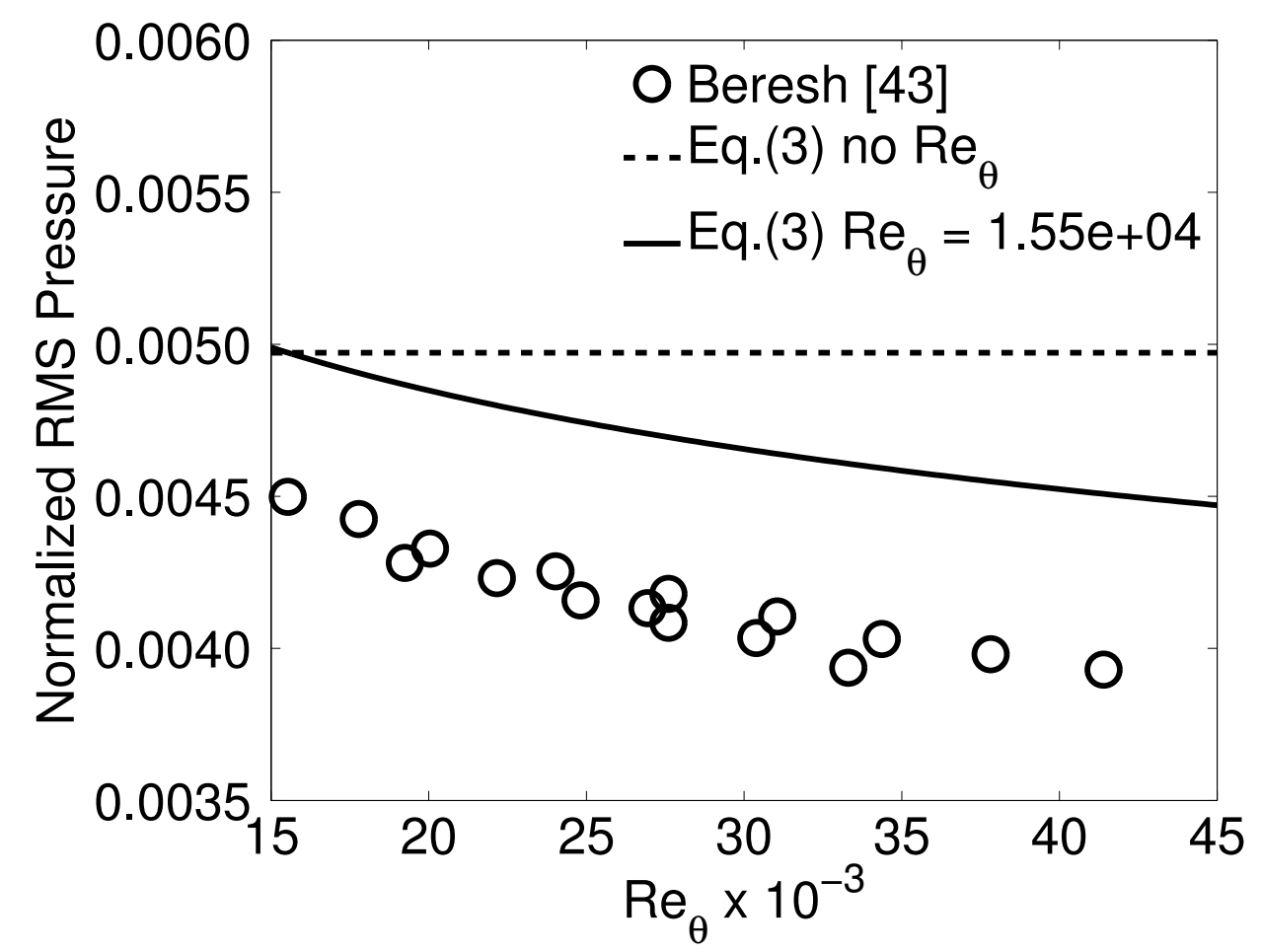

(a) Mach 2.5.

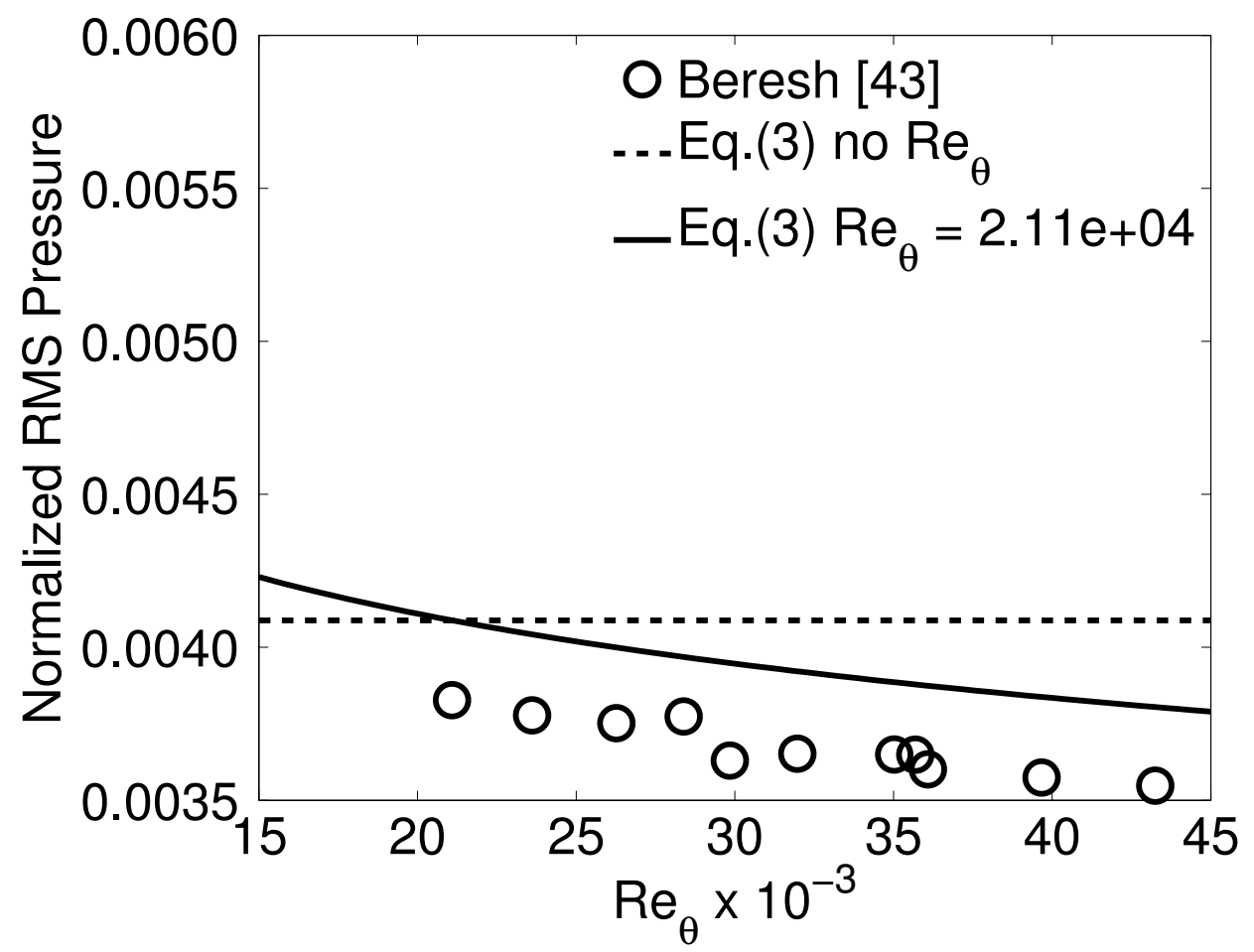

(b) Mach 3.0.

Figure 2.7: Turbulent boundary-layer fluctuating pressure model comparison with the experimental data of Beresh et al. [142]. RMS pressure normalized by dynamic pressure at boundary-layer edge. 


\subsubsection{Eckert's Reference Enthalpy}

Eckert's reference enthalpy method [134] predicts skin friction and heat transfer for hypersonic flows by evaluating incompressible boundary layer relations at a reference condition that corrects for compressibility effects. Eckert's relation for the reference enthalpy of a boundary layer is given as Eq. (2.31).

$$
H^{*}=0.5\left(H_{e}+H_{w}\right)+0.22 H_{e} r \frac{\gamma-1}{2} M_{e}^{2}
$$

Where $H^{*}, H_{e}$, and $H_{w}$ are the reference, boundary-layer edge, and wall enthalpies and $r$ is the recovery factor. The recovery factor is defined in Eq. (2.32) for laminar and turbulent flow, where $\operatorname{Pr}$ is the Prandtl number. The Mach number and enthalpy at the boundary-layer edge are determined using the pressure obtained from piston theory in conjunction with isentropic flow relations. Therefore, the panel dynamics are accounted for in the aerodynamic heating prediction.

$$
r= \begin{cases}\left(P r^{*}\right)^{1 / 2} & \text { (laminar) } \\ \left(P r^{*}\right)^{1 / 3} & \text { (turbulent) }\end{cases}
$$

Reference flow properties $\left(T^{*}, \rho^{*}, \mu^{*}\right)$ are obtained through temperature-enthalpy tables that include the effect of dissociation based on equilibrium air properties, the ideal gas law, and Sutherland's law. The aerodynamic heat flux is computed from Eq. (2.33) using the flow properties evaluated at the reference condition, wall, and 
boundary-layer edge. The Stanton number $(S t)$ is computed using the ColburnReynolds analogy in Eq. (2.34). The local skin friction coefficient is obtained from Eq. (2.35), where the laminar relation is the Blasius solution for a flat plate boundary layer and the turbulent relation is the Schultz-Grunow formula [111].

$$
\begin{gathered}
Q(x)=S t^{*} \rho^{*} U_{e}\left(H_{a w}-H_{w}\right) \\
S t^{*}=\frac{c_{f}^{*}}{2}\left(P r^{*}\right)^{-2 / 3} \\
c_{f}^{*}=\left\{\begin{array}{rr}
0.664\left(R e_{x}^{*}\right)^{-1 / 2} & \text { (laminar) } \\
0.370\left(\log _{10} R e_{x}^{*}\right)^{-2.584} & \text { (turbulent) } \\
R e_{x}^{*}=\frac{\rho^{*} U_{e} x}{\mu^{*}} &
\end{array}\right.
\end{gathered}
$$

\subsubsection{Transitional Aerothermodynamic Loads}

The aerothermodynamic loads acting on the panel are heat flux and an overall pressure load (composed of a mean and fluctuating component). Transitional boundary layer effects are incorporated into the heat flux and fluctuating pressure through blending laminar and turbulent profiles in proportion to an intermittency function. The intermittency represents the fraction of time any spatial location spends in turbulent flow [143]. A brief description of the intermittency function is given in Section 2.3.3.1. Details on the transitional heat flux and fluctuating pressure models, along with experimental comparisons, are provided in 
Sections 2.3.3.2-2.3.3.4.

\subsubsection{Intermittency}

Transition from laminar to turbulent flow is, in general, not an abrupt process. It occurs over a finite length due to the growth, propagation, and interaction of turbulent spots [144]. As a result, the flow during transition can be characterized as a laminar boundary layer subject to intermittent patches of turbulence. Thus, the statistical flow properties in the transition region can be described using an intermittency function that represents the fraction of time any spatial location spends in turbulent flow. The intermittency throughout the transition region is computed using Eq. (2.37), which is derived from Emmon's probabilistic model [144, 145] with the assumption that the burst source-rate density is described as a Dirac delta function.

$$
\gamma(x)=1-\exp \left[-\frac{\sigma n}{u_{e}}\left(x-x_{t}\right)^{2}\right]
$$

This form of the source-rate density function assumes the hypothesis of concentrated breakdown is valid [24], implying that turbulent spots are formed only at the transition onset location $x_{t}$. However, at the transition onset location, turbulent spots may form randomly in time and in the spanwise direction. The intermittency distribution in Eq. (2.37) is a function of the edge velocity $u_{e}$ (assumed constant over the transition region), the transition onset location $x_{t}$, the number

of turbulent spots per unit time and spanwise distance $n$, and a spot propaga- 
tion parameter $\sigma$. The spot propagation parameter is defined in Eq. (2.38), where $u_{g}=u_{l}-u_{t}, u_{c}=1 / 2\left(u_{l}+u_{t}\right), u_{l}$ and $u_{t}$ are the leading- and trailing-edge velocities of the spot, and $\alpha$ is the half-angle of the turbulent spot.

$$
\sigma=\frac{u_{g} u_{e}}{u_{c} u_{t}} \tan \alpha
$$

The length of the transition region $\Delta x_{t}$ is defined as the spatial distance between intermittency values of 0.01 and 0.99 . By solving Eq. (2.37) for $\left(x-x_{t}\right)$ at $\gamma=$ 0.01 and 0.99 , the transition length is expressed as shown in Eq. (2.39).

$$
\Delta x_{t}=\left.\left(x-x_{t}\right)\right|_{\gamma=0.99}-\left.\left(x-x_{t}\right)\right|_{\gamma=0.01}=2.0457 \sqrt{\frac{u_{e}}{\sigma n}}
$$

Introducing $\Delta x_{t}$ into the exponent in Eq. (2.37), results in an expression for the intermittency that is a function of the transition onset and length alone:

$$
\gamma(x)=1-\exp \left[-\frac{4.1850}{\Delta x_{t}^{2}}\left(x-x_{t}\right)^{2}\right]
$$

\subsubsection{Heat Flux}

The transitional heat flux is approximated by using the intermittency to blend the laminar and turbulent values as shown in Eq. (2.41):

$$
Q_{\text {tran }}=(1-\gamma) Q_{\text {lam }}+\gamma Q_{\text {turb }_{V O}}
$$


If the laminar and turbulent boundary layers originate at the same location, the blending in Eq. (2.41) cannot account for transitional overshoot in heat flux [24]. Overshoot can be incorporated by assuming the turbulent boundary layer begins at a virtual origin corresponding to the transition onset location [143]. Applying the linear blending in Eq. (2.41) and assuming the turbulent boundary layer originates at $x_{t}$, Dhawan and Narasimha [143] matched experimental skin friction coefficients and displacement thickness during transition.

Previous experiments that have observed transitional overshoot in heat flux [25] indicate that heating rates decrease back to fully turbulent conditions beyond the overshoot region. To model this, the transitional heat flux [computed using Eq. (2.41)] is blended with the fully turbulent heating rates as shown in Eq. (2.42), where $\Lambda$ is a Gaussian function, defined in Eq. (2.43).

$$
\begin{gathered}
Q(x)=(1-\Lambda) Q_{t u r b}+\Lambda Q_{t r a n} \quad\left(x_{t e} \leq x \leq x_{e n d}\right) \\
\Lambda(x)=\exp \left[\frac{-\left(\left(x-x_{t e}\right) /\left(x_{e n d}-x_{t e}\right)\right)^{2}}{2 \beta^{2}}\right]
\end{gathered}
$$

The peak of the Gaussian function coincides with the end of transition $x_{t e}$. Specifying the standard deviation as shown in Eq. (2.44) results in the Gaussian function decreasing to negligible values $\mathcal{O}\left(10^{-5}\right)$ by the end of the geometry $x_{\text {end }}$. This 
ensures that fully turbulent heating rates are obtained on the end of the geometry.

$$
\beta=\frac{0.5}{2 \sqrt{2 \ln 2}}
$$

An example of the transitional heat flux generated using this model is provided in Fig. 2.8 for transition beginning at $\bar{x}=0.3$ and ending at $\bar{x}=0.5$, where $\bar{x}$ corresponds to $x / L$. The "No overshoot" line in Fig. 2.8 corresponds to the transitional heat flux profile obtained using Eq. (2.41) if the laminar and turbulent boundary layers have the same origin. The "Overshoot" heat flux was generated assuming the turbulent boundary layer originates at $\bar{x}=0.3$ with the Gaussian blending applied in the turbulent region ( $\bar{x} \geq 0.5)$. The heat flux profiles in Fig. 2.8 demonstrate that, through shifting the turbulent boundary layer origin, this model can be used to generate heat flux profiles that either account for or neglect the effect of transitional overshoot.

\subsubsection{Fluctuating Pressure}

The turbulent boundary layer RMS pressure expression, in Eq. (2.27), is modified to account for the spatial variation in RMS resulting from transitional flow. The modified expression [Eq. (2.45)] incorporates a dependence on the local skinfriction coefficient. Here, $c_{f_{t r a n}}$ and $c_{f_{\text {turb }}}$ are the local skin-friction coefficients cor- 


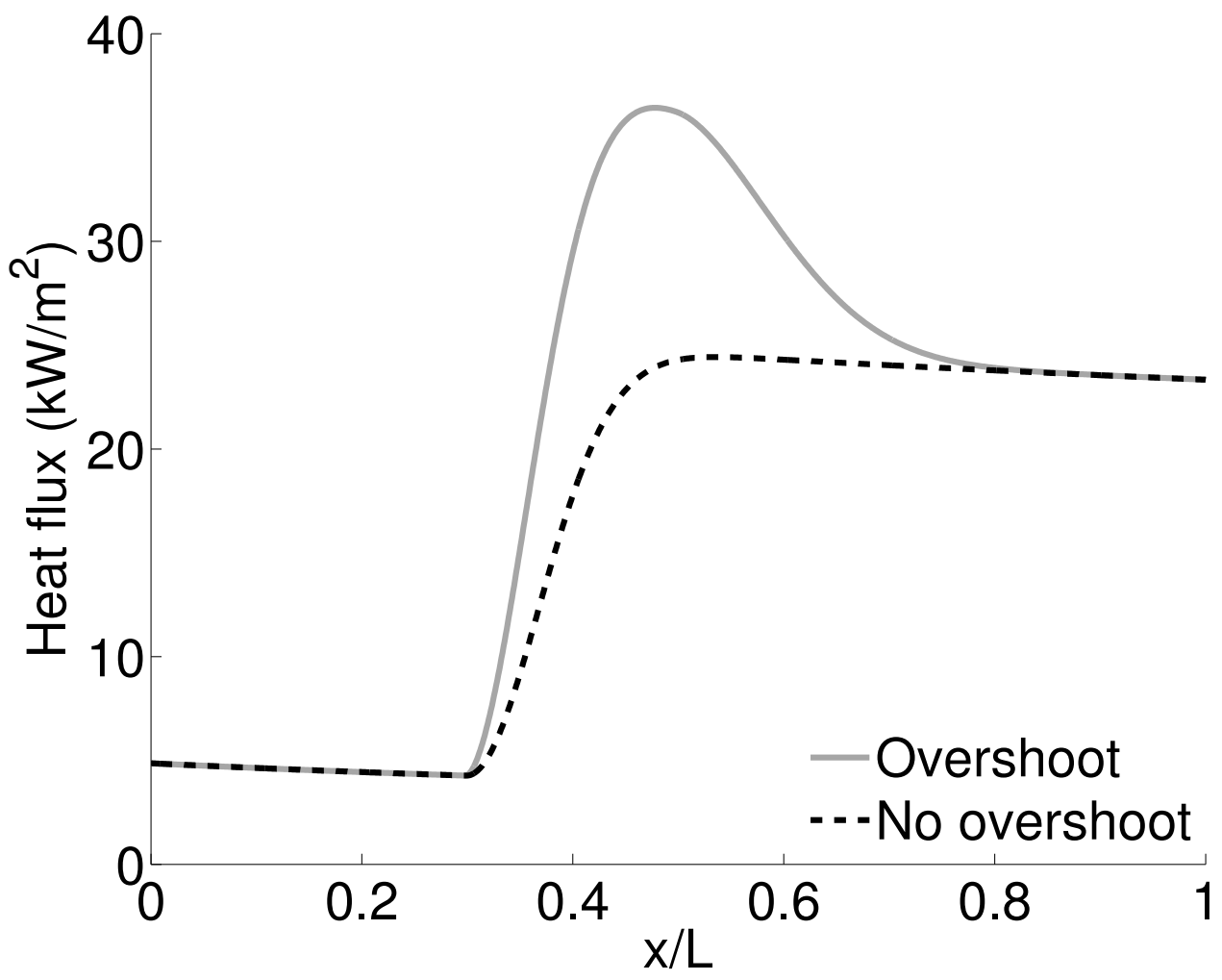

Figure 2.8: Transitional heat flux model. Transition beginning at $\bar{x}_{t}=0.3$ and ending at $\bar{x}_{t e}=0.5$. 
responding to transitional and fully turbulent boundary layers:

$$
\frac{\tilde{p}}{q_{e}}=0.009 F_{c}^{\lambda(1+b)}\left(\frac{R e_{\theta}}{\left.R e_{\theta}\right|_{\bar{x}^{*}}}\right)^{-0.1} \frac{c_{f_{\text {tran }}}}{c_{f_{\text {turb }}}}
$$

The relationship in Eq. (2.45) is similar to the Laganelli model for turbulent boundary-layer pressure fluctuations on rough surfaces [18], which scales the smoothwall RMS pressure using the skin-friction ratio to obtain the rough-wall RMS pressure. The transitional skin-friction coefficient is computed in the same manner as the heat flux, as shown in Eq. (2.46) again using the Gaussian smoothing in the turbulent region. To remove leading-edge effects, the RMS pressure in the laminar region is specified as the minimum RMS value prior to transition onset.

$$
c_{f_{\text {tran }}}=(1-\gamma) c_{f_{\text {lam }}}+\gamma c_{f_{\text {turb }} \text { O }}
$$

Previously developed correlations to predict transitional pressure fluctuations $[139,146]$ account for spatial variation in the RMS magnitude through varying the velocity power law exponent, which is used to define the exponent of $F_{c}$ in Eq. (2.45). Laganelli and Howe [139] provided discrete values for the velocity power law exponent as $n=4$ for the start to the middle of transition, $n=6$ for the peak transition values, and $n=7$ for turbulent flow. However, as noted by Casper [147], the Laganelli transitional RMS correlation is inconsistent, as $n=4$ predicts higher pressure fluctuations than $n=6$. 
An example of the transitional pressure load is provided in Fig. 2.9 in terms of the RMS pressure (Fig. 2.9.a) and the minimum and maximum values of the fluctuating pressure during the first $0.5 \mathrm{~s}$ of response (Fig. 2.9.b). As with the heat flux profiles in Fig. 2.8, results are presented for shifted and unshifted turbulent boundary layers to highlight the effect of accounting for or neglecting transitional overshoot. The RMS pressure in Fig. 2.9.a demonstrates that the present formulation provides a smooth spatial variation in the RMS pressure throughout transition, with peak magnitudes occurring at the end of transition. The fluctuating pressure envelopes in Fig. 2.9.b indicate that accounting for overshoot increases the intensity of the pressure load acting on the panel throughout the transition region.

\subsubsection{Experimental Comparisons}

Transitional heat transfer rates, predicted using the model in Section 2.3.3.2, are compared against experimental values measured on a full-scale HIFiRE-1 flight test program geometry in the CUBRC LENS I hypervelocity reflected shock tunnel [25, 121] in Fig. 2.10. The experimental measurements (run 4 of [121]) in Fig. 2.10 correspond to Mach 6.58 flow over a $7^{\circ}$ cone with a $2.5 \mathrm{~mm}$ nosetip. Laminar and fully turbulent heat flux predictions (labeled LAM and TURB) are provided in Fig. 2.10 to highlight the overshoot in the transitional heat flux. The transitional heat flux curve (labeled TRAN) assumes transition onset at $x_{t}=0.35 \mathrm{~m}$ with a transition length of $\Delta x_{t}=0.21 \mathrm{~m}$. The transitional heat flux model provides rea- 


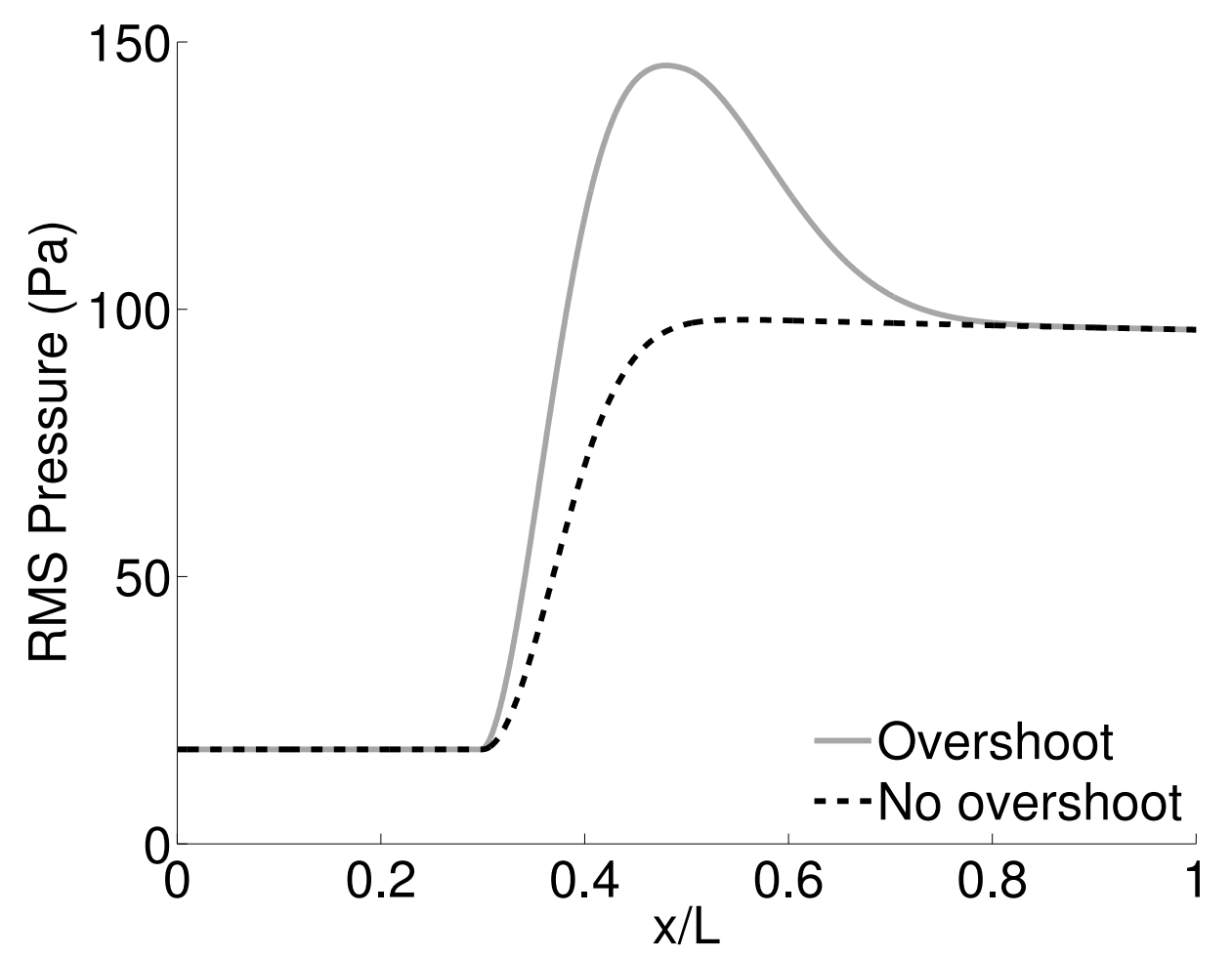

(a) RMS of fluctuating pressure.

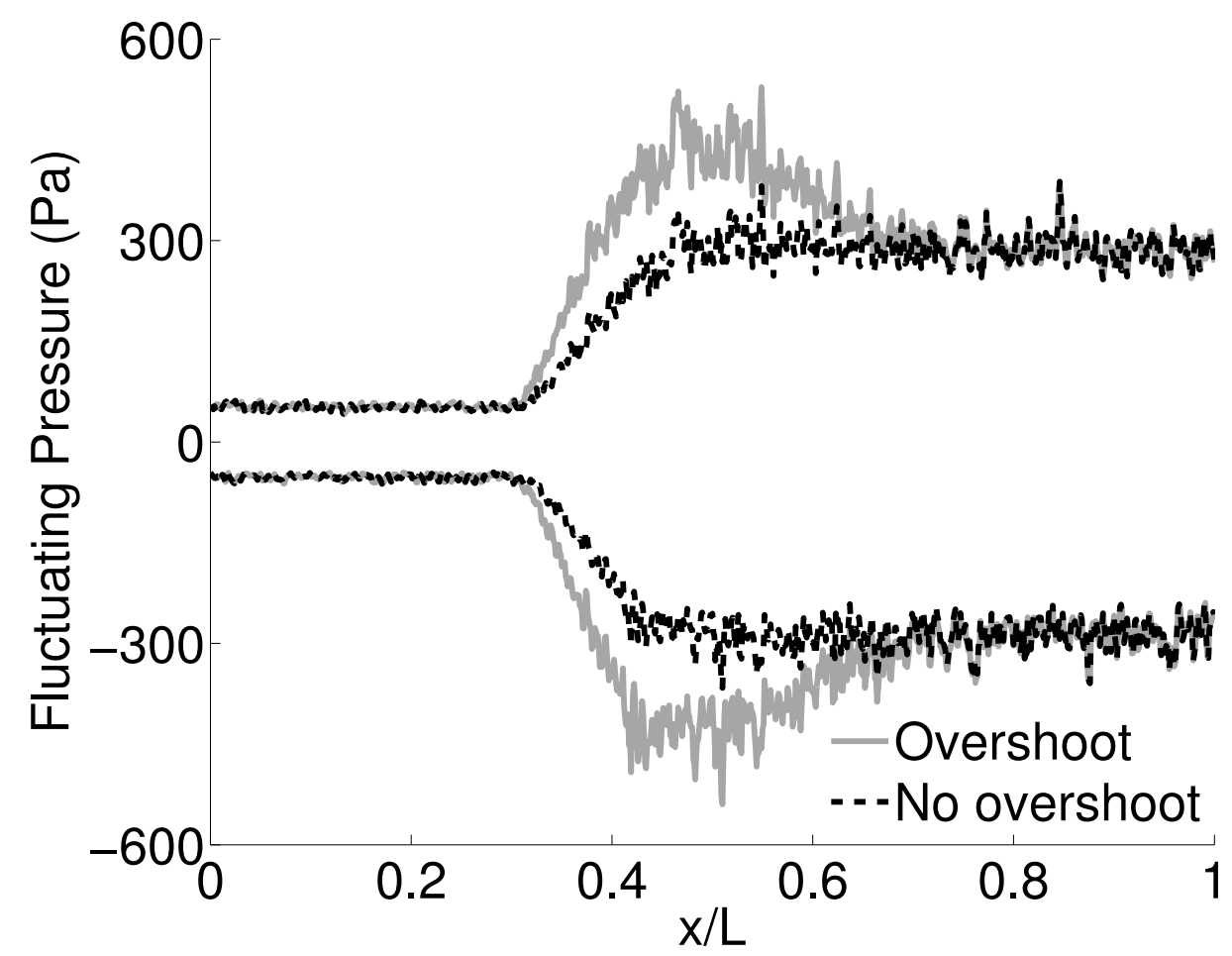

(b) Fluctuating pressure envelope.

Figure 2.9: Transitional fluctuating pressure load model. Transition beginning at $\bar{x}_{t}=0.3$ and ending at $\bar{x}_{t e}=0.5$. 


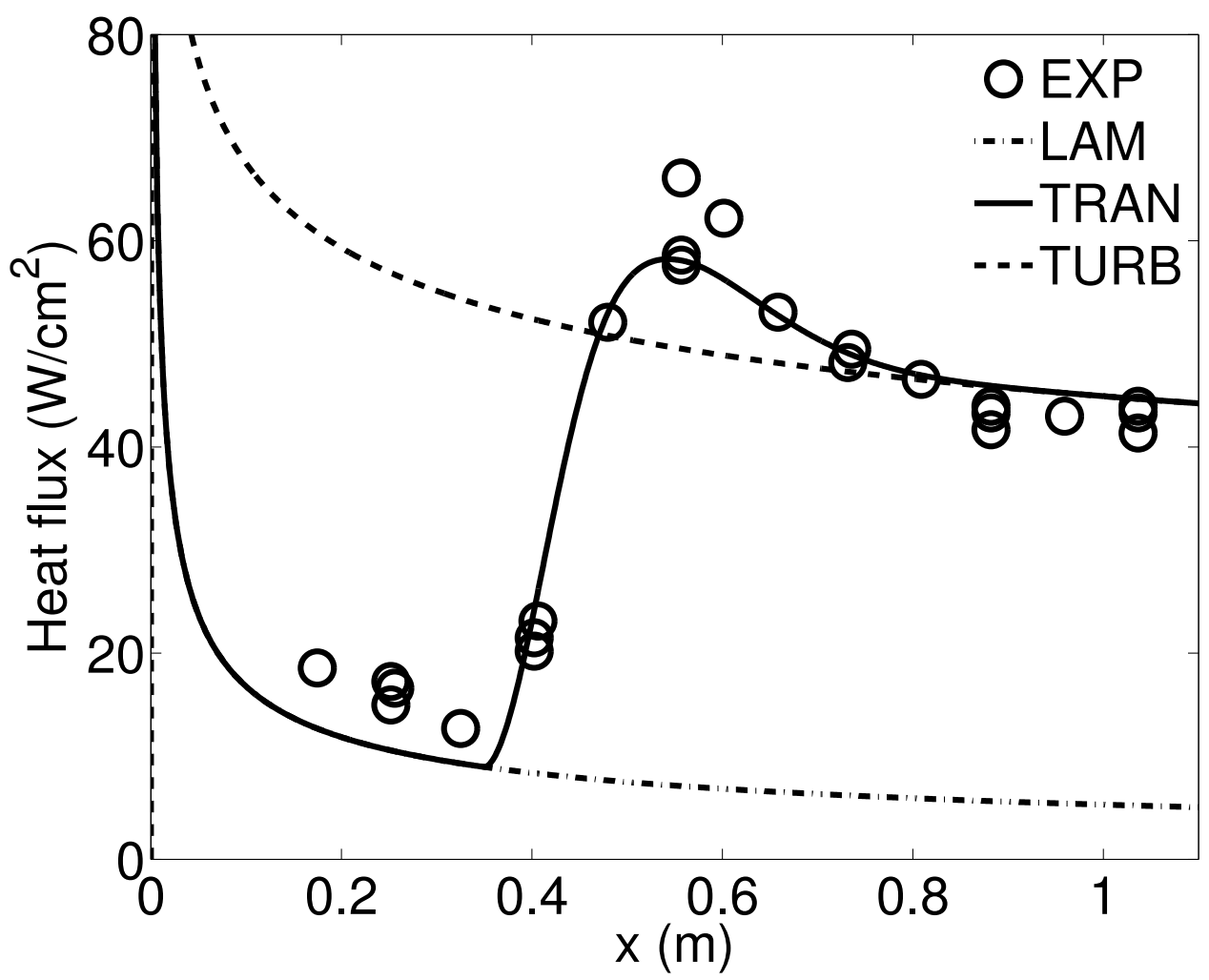

Figure 2.10: Transitional heat flux for $x_{t}=0.35 \mathrm{~m}$ and $\Delta x_{t}=0.21 \mathrm{~m}$ (experimental data digitized from figure 17a of [121]).

sonable agreement with the experimental data (labeled EXP) and is comparable with Reynolds-averaged Navier-Stokes-based predictions provided in [121].

Next, to assess the capability of the transitional fluctuating pressure model, RMS pressure and PSD predictions are compared against the experimental data of Casper et al. [29], obtained in the Sandia Hypersonic Wind Tunnel. The predicted RMS (Fig. 2.11) and PSD (Fig. 2.12) values correspond to Mach 5 flow $(R e / m=$ $15.4 \times 10^{6}$ ) over a $7^{\circ}$ half-angle cone with transition onset at $x_{t}=0.20 \mathrm{~m}$ and a transition length of $\Delta x_{t}=0.084 \mathrm{~m}$. RMS predictions are provided in Fig. 2.11 for both turbulent [Eq. (2.27)] and transitional flow [Eq. (2.45)], where $\left.\operatorname{Re}_{\theta}\right|_{\bar{x}^{*}}$ was 
obtained at $x=0.2 \mathrm{~m}$. Comparing the transitional and experimental RMS values in Fig. 2.11 illustrates that the model under predicts the experimental data. This could be due, in part, to noise radiated from the nozzle wall of the wind tunnel that biases the experimental RMS levels higher than the model [147]. Despite the discrepancy in magnitude, the model captures the rise and peak in RMS pressure, during transition, as well as the reduction back to turbulent conditions.

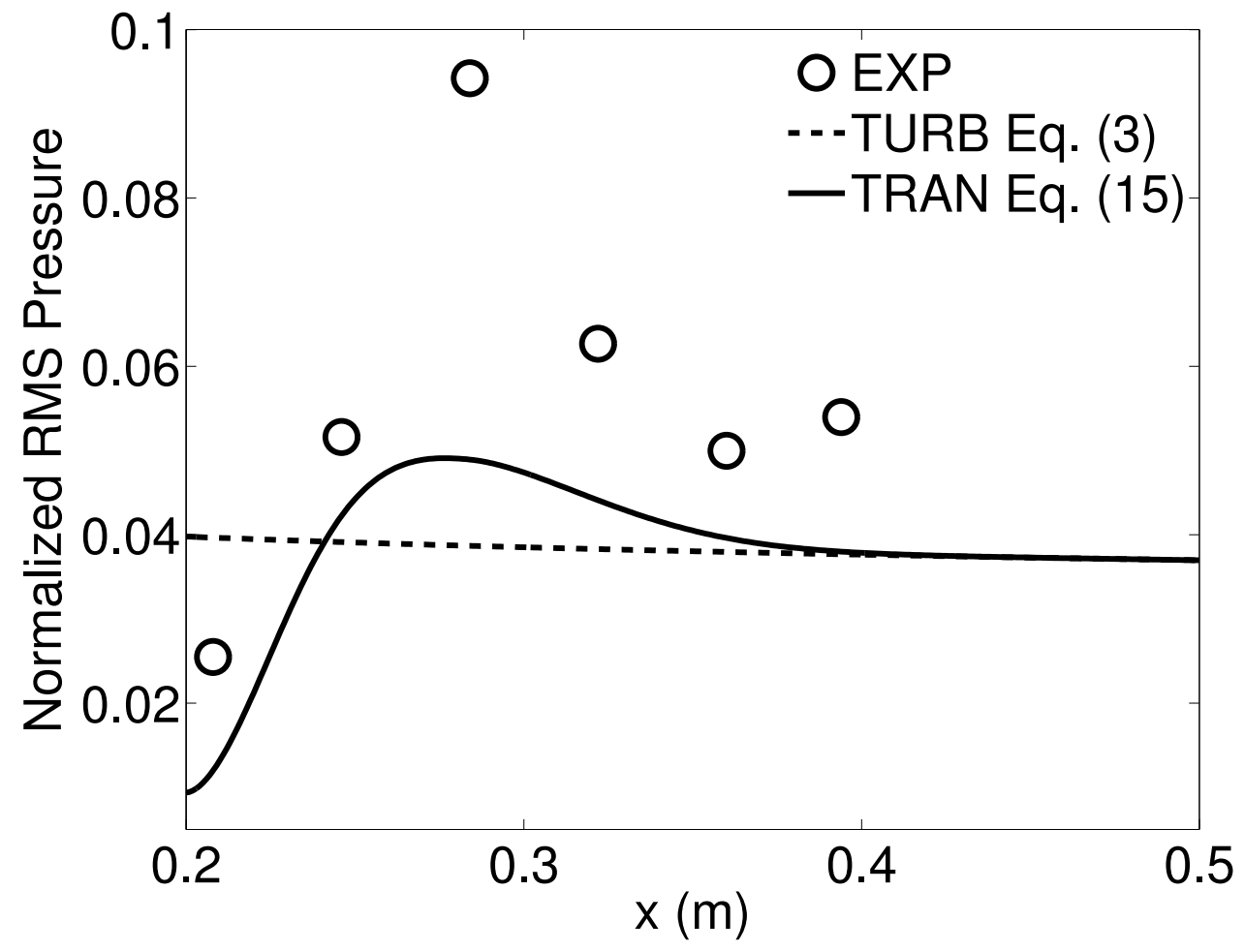

Figure 2.11: Transitional RMS pressure normalized by boundary-layer edge pressure for $x_{t}=0.20 \mathrm{~m}$ and $\Delta x_{t}=0.084 \mathrm{~m}$ (experimental RMS pressure values are totals between 0 and $1 \mathrm{MHz}$ from [29]).

Experimental and modeled PSDs are provided at three locations in Fig. 2.12 corresponding to early transitional flow $(x=0.208 \mathrm{~m})$, the RMS pressure peak $(x=0.284 \mathrm{~m})$, and the turbulent region $(x=0.398 \mathrm{~m})$. For the modeled PSDs 
in Fig. 2.12, the largest magnitude corresponds to the location of the peak RMS value, which agrees with experimental observations [28, 29]. As the PSD expression in Eq. (2.28) was originally developed for turbulent boundary layers, it fails to capture the second-mode waves experimentally observed between $x=0.208$ $\mathrm{m}$ and $x=0.246 \mathrm{~m}$. Further, the modeled PSDs under-predict the power associated with low-frequency pressure fluctuations that are most important to the structural response. This is adjusted through the PSD scaling required to reproduce the fluctuating pressure signal for a truncated frequency domain, as described in Section 2.3.2.2.

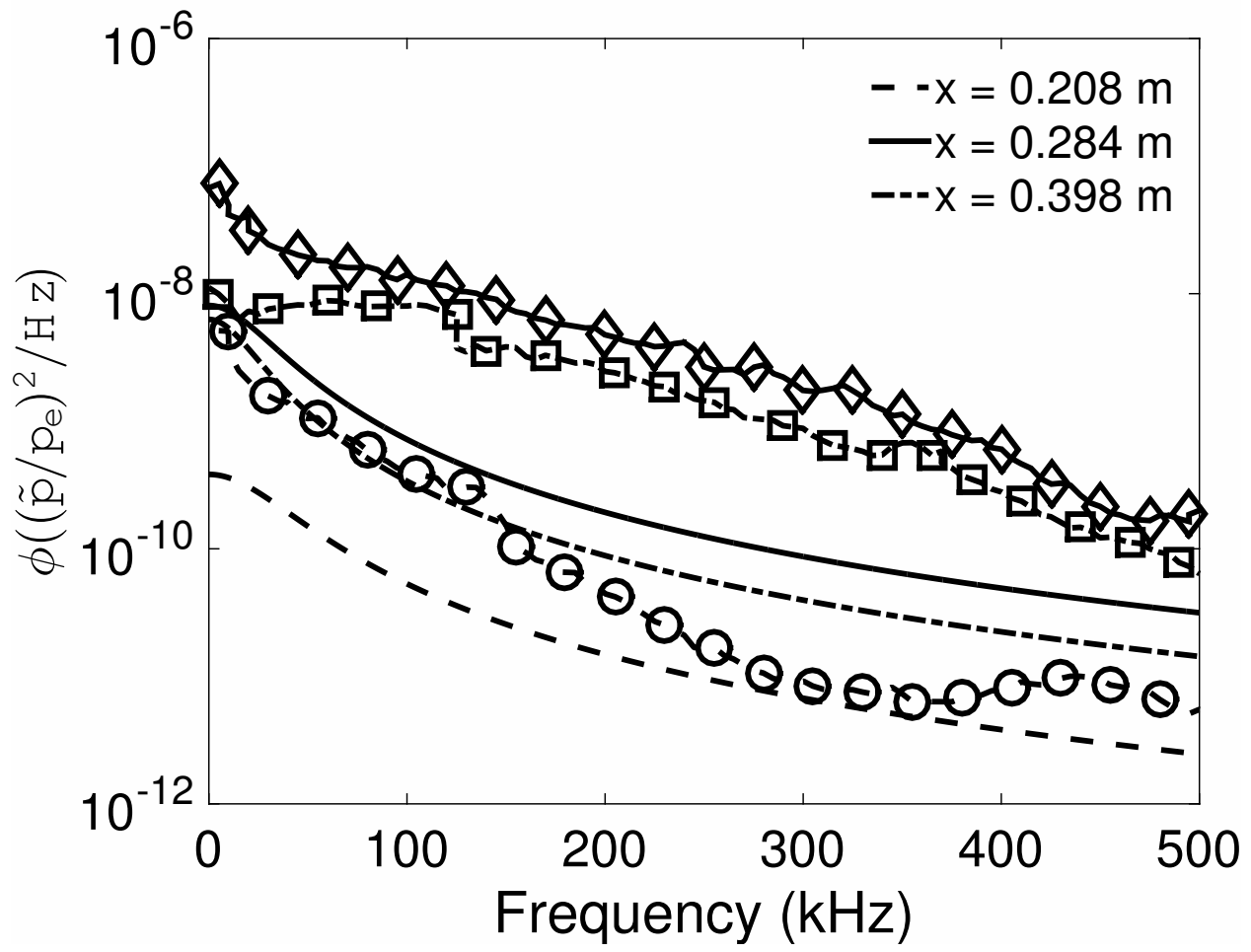

Figure 2.12: Transitional PSDs for $x_{t}=0.20 \mathrm{~m}$ and $\Delta x_{t}=0.084 \mathrm{~m}$. Lines with markers denote experimental data digitized from figure $9 c$ of [29]. 


\subsubsection{Thermal Model}

The thermal model is depicted in Fig. 2.13. An adiabatic wall condition is prescribed for each boundary of the panel, except the upper surface where heat flux is applied. The surface heat flux either accounts for: 1) purely aerodynamic heating or 2) both aerodynamic heating and radiation between the structure and the freestream.

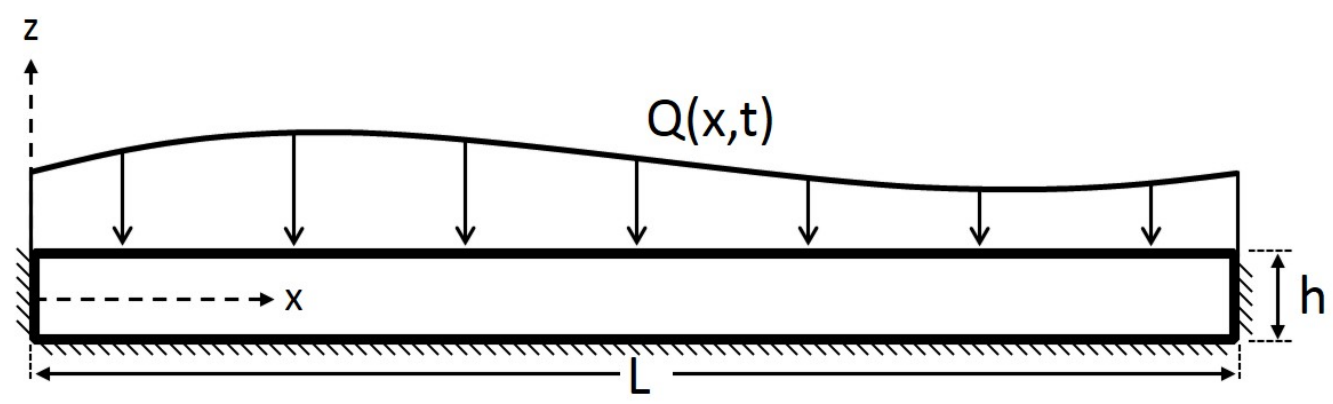

Figure 2.13: Thermal Model.

The temperature throughout the panel is obtained by solving a finite element formulation of the transient, 2-D heat transfer equation in Eq. (2.47).

$$
\rho c(x, z, T) \frac{\partial T(x, z, t)}{\partial t}-\nabla \cdot(\kappa(x, z, t) \nabla T(x, z, t))=0
$$

The thermal model accounts for temperature-dependent specific heat $(c)$ and thermal conductivity $(\kappa)$. Additionally, the 2-D formulation allows for heat conduction through both the thickness and length of the panel. The thermal boundary 
conditions are provided in Eq. (2.48).

$$
\begin{aligned}
\left.\kappa \frac{\partial T}{\partial z}\right|_{z=h / 2} & =Q(x, t) \\
\left.\frac{\partial T}{\partial x}\right|_{x=0, L} & =\left.\frac{\partial T}{\partial z}\right|_{z=-h / 2}=0
\end{aligned}
$$

The finite element discretization consists of four node quadrilateral elements using linear shape functions of the temperature [148]. The thermal model is discretized with 4 elements through the thickness and either 1000 (Chapter 4) or 1500 (Chapter 5) elements along the length of the panel. Miller et al. [148] verified the thermal model against an Abaqus ${ }^{\circledR}$ model of equal size, for the case of a constantin-time, piecewise heat flux profile that linearly increases and decreases about the mid-chord of the panel. The maximum temperature difference for $10 \mathrm{~s}$ of response was less than $0.006 \mathrm{~K}$.

\subsubsection{Structural Model}

The structural model is depicted in Fig. 2.14. The panel is subject to aerodynamic pressure on the top surface and a prescribed back pressure on the bottom surface. Both simply-supported/pinned and clamped boundary conditions are considered. In either case, the boundaries are fully constrained in $x$ and $z$ translation. The structural model only considers the transverse displacement in the $z$ direction. 


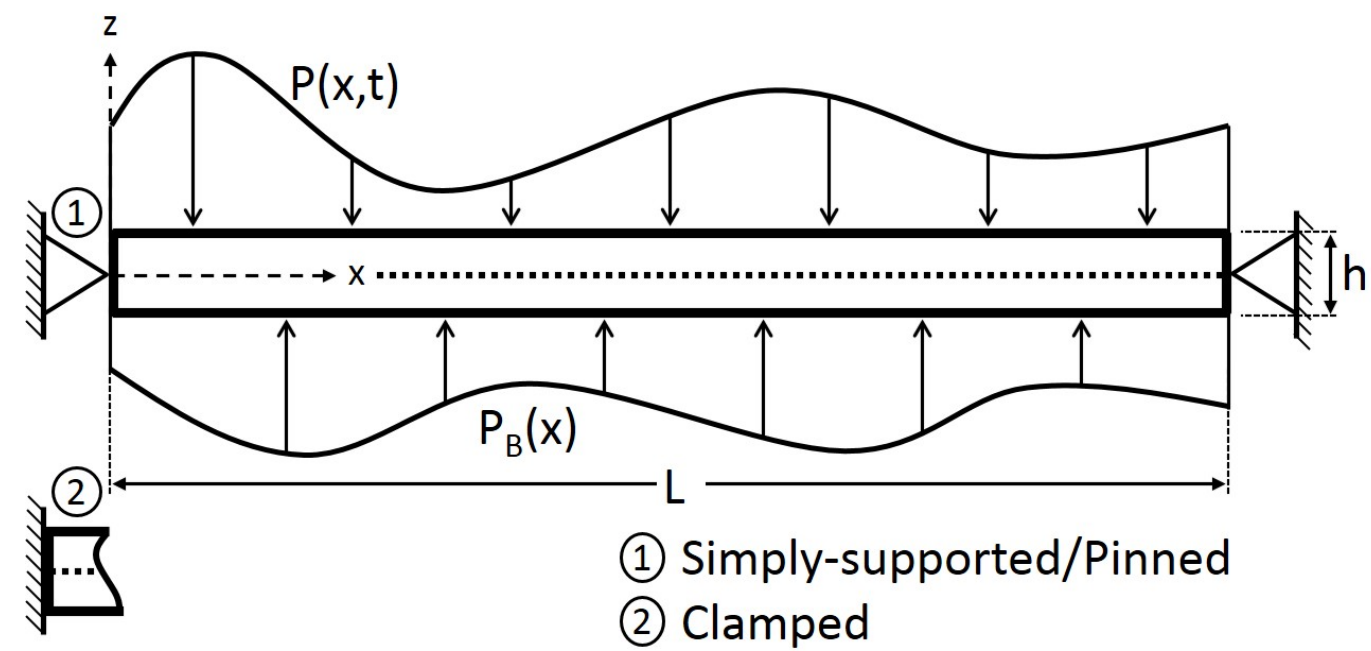

Figure 2.14: Structural Model.

The structure is modeled as cylindrical bending of an isotropic plate with the assumptions of von Kármán moderate deflection plate theory [33]. The formulation includes the effects of thermal loading due to non-uniform (in-plane and through-thickness) temperature distributions, chordwise variation of the modulus of elasticity and thermal expansion coefficient, rotary inertia, and Rayleigh damping. The equation of motion is given by Eq. (2.49) in conjunction with Eqs. (2.50)(2.53), where $m$ is the mass per unit length, $w$ is the transverse displacement, $I$ is the cross-sectional mass moment of inertia, $\bar{C}$ is the material damping, $D$ is the bending stiffness, $N_{x}$ is the overall in-plane force, $N_{T}$ is the in-plane thermal force, $P_{n e t}$ is the net pressure load $\left(P_{B}-P\right)$, and $M_{T}$ is the thermal moment.

$$
\begin{array}{r}
\frac{\partial^{2}}{\partial t^{2}}\left(m w(x, t)-I \frac{\partial^{2} w(x, t)}{\partial x^{2}}\right)+\bar{C} \frac{\partial w(x, t)}{\partial t}+\frac{\partial^{2}}{\partial x^{2}}\left(D(x, t) \frac{\partial^{2} w(x, t)}{\partial x^{2}}\right) \\
-N_{x}(x, t) \frac{\partial^{2} w(x, t)}{\partial x^{2}}=P_{n e t}(x, t)-\frac{\partial^{2} M_{T}(x, t)}{\partial x^{2}}
\end{array}
$$




$$
\begin{gathered}
D=\frac{E(x, t) h^{3}}{12\left(1-\nu^{2}\right)} \\
N_{x}=\frac{E(x, t) h}{1-\nu^{2}}\left[\frac{\partial u_{0}}{\partial x}+\frac{1}{2}\left(\frac{\partial w}{\partial x}\right)^{2}\right]-N_{T}(x, t) \\
N_{T}=\frac{E(x, t) \alpha(x, t)}{1-\nu} \int_{-h / 2}^{h / 2}\left[T(x, z, t)-T_{r e f}\right] d z \\
M_{T}=\frac{E(x, t) \alpha(x, t)}{1-\nu} \int_{-h / 2}^{h / 2}\left[T(x, z, t)-T_{r e f}\right] z d z
\end{gathered}
$$

Following the procedure outlined in [33], the in-plane displacement term $\left(\frac{\partial u_{0}}{\partial x}\right)$ is eliminated from Eq. (2.51). This results in the in-plane stress resultant in Eq. (2.54), which is a function of the transverse displacement and average thermal force in Eq. (2.55). The expression in Eq. (2.55) physically implies that the in-plane thermal effects are primarily driven by the chordwise average temperature [35].

$$
\begin{array}{r}
N_{x}=\left[\int_{0}^{L} \frac{1}{E(x, t)} d x\right]^{-1} \int_{0}^{L} \frac{h}{2\left(1-\nu^{2}\right)}\left(\frac{\partial w}{\partial x}\right)^{2} d x-\overline{N_{T}}(t) \\
\overline{N_{T}}=\left[\int_{0}^{L} \frac{1}{E(x, t)} d x\right]^{-1} \int_{0}^{L} \frac{\alpha(x, t)}{1-\nu} \int_{-h / 2}^{h / 2}\left[T(x, z, t)-T_{r e f}\right] d z d x
\end{array}
$$

The boundary conditions required to solve the equation of motion are provided in Eqs. (2.56)-(2.57) for both the simply-supported and clamped panel, respectively.

$$
\begin{aligned}
w(0, t)=w(L, t) & =0 \\
\left.\frac{\partial^{2} w}{\partial x^{2}}\right|_{x=0}=\left.\frac{\partial^{2} w}{\partial x^{2}}\right|_{x=L} & =-\frac{M_{T}(x, t)}{D(x, t)}
\end{aligned}
$$




$$
\begin{aligned}
w(0, t) & =w(L, t)=0 \\
\left.\frac{\partial w}{\partial x}\right|_{x=0}=\left.\frac{\partial w}{\partial x}\right|_{x=L} & =0
\end{aligned}
$$

The dependence of the natural boundary condition on the thermal moment [Eq. (2.56b)] biases the simply-supported panel to buckle into the flowfield when heated from the top surface. The use of boundary conditions that better represent the attachment of surface panels to the vehicle substructure would impact the structural response. For instance, allowing for axial expansion reduces the resistance to in-plane thermal expansion which decreases the panel deformation and the importance of the aerothermoelastic coupling [101]. However, pinned boundary conditions have been extensively examined in past works [17, 35] and produce a highly-coupled panel response over a relatively short time record. Clamped boundary conditions are also considered in this work to examine the response of a stiffer panel that is less sensitive to the average temperature rise.

The structural equation of motion is discretized using Galerkin's method. The transverse displacement is approximated as a series of free-vibration mode shapes $\left(\phi_{i}\right)$ that satisfy either pinned or clamped boundary conditions:

$$
w(x, t) \approx \sum_{i=1}^{N} a_{i}(t) \phi_{i}(x)
$$


where $a_{i}$ are the modal weights, and $N$ is the number of modes used to approximate the displacement.

For the results in Chapter 4 and Chapter 5, 25 free-vibration mode shapes are used to approximate the transverse displacement of the panel. The discretized mode shapes have a consistent spatial step size of $1 \mathrm{~mm}$, resulting in 1001 points for the $1.0 \mathrm{~m}$ panel examined in Chapter 4 and 1501 points for the $1.5 \mathrm{~m}$ panel studied in Chapter 5. Additionally, the mass and stiffness-proportional Rayleigh damping is specified such that $2 \%$ of the critical damping is achieved at both the fundamental natural frequency and at $1000 \mathrm{~Hz}$ to provide viscous damping of the higher frequencies. The structural model has been shown to provide good agreement with an Abaqus ${ }^{\circledR}$ model consisting of 1000 cubic-interpolation beam elements for the cases of a spatially-random pressure loading and uniformly-rising panel temperature [148].

\subsection{Generic Panel Configuration}

The interaction between boundary-layer transition and the aerothermoelastic response of a panel on the surface of a hypersonic vehicle is examined using the configuration depicted in Fig. 2.15. For each study, it is assumed that a compliant panel is located on a 2-D surface inclined $5^{\circ}$ to the freestream. The flow the panel experiences corresponds to the post oblique shock conditions. Note that, while the panel in Fig. 2.15 is shown to be simply-supported, clamped structural boundary 
conditions are also examined in this work. The state of the boundary layer (laminar, transitional, or turbulent) developing over the surface is either: predicted based off prescribed surface conditions (Chapter 3), prescribed to examine its effect on the structural response (Chapter 4), or allowed to vary in time as a function of the structural response (Chapter 5). Details on the freestream conditions as well as panel location, geometry, and material are provided along with the results in Chapters 3-5.

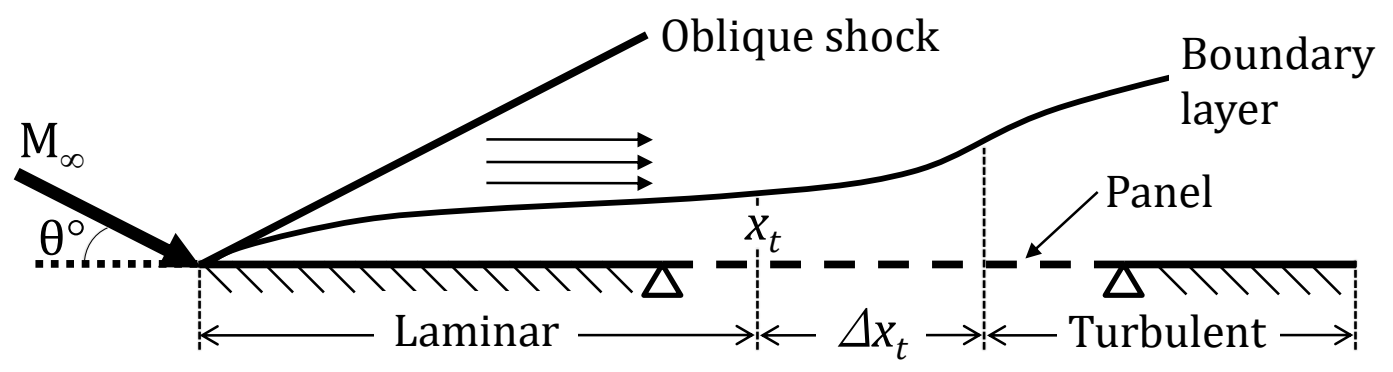

Figure 2.15: Compliant panel located on 2-D surface inclined to freestream. 


\section{Chapter 3}

\section{Impact of Surface Conditions on Hypersonic}

\section{Boundary-Layer Stability}

The boundary-layer stability of flow past large-scale, 2-D surface deformations is assessed using linear stability theory and the linear parabolized stability equations. The deformations are obtained from previous research $[35,93,101]$ and represent the characteristic response of integrated thermal protection system panels on a hypersonic vehicle. The deformations are placed on the surface of a 2-D, sharp leading edge, wedge geometry similar to that shown in Fig. 2.15. Boundary-layer stability analysis is performed to examine the effect of buckling direction, deformation location, multiple deformations in series, structural boundary condition, surface temperature, the combined effect of Mach number and altitude, and deformation mode shape. The deformations extracted from $[35,93,101]$ are described in Section 3.1. Grid convergence of the laminar, steady-state flow solutions is demonstrated in Section 3.2. Results pertaining to each deformation are presented in 
Section 3.3.

\subsection{Panel Geometries}

Three different panel configurations, based on previous work, are selected for the present study. The 2-D deformation, wedge geometry and freestream conditions used for each case are briefly described next. Relevant nondimensional parameters that describe the deformations are also provided.

\subsubsection{Generic Metallic Panel}

Miller et al. [35] assessed the impact of flow induced loads on the behavior of an acoustically excited, thermally buckled, 2-D metallic panel. The deformation and temperature profiles in Fig. 3.1 are extracted from [35] to examine the effect of deformation direction, panel location, and multiple panel arrangements on boundary-layer stability. The freestream conditions and wedge geometry are detailed in Table 3.1. For the generic metallic panel, the wedge is assumed to be at a uniform surface temperature $\left(T_{I S O}\right)$ of $300 \mathrm{~K}$ and the wall temperature over the panel matches the profiles in Fig. 3.1.

\subsubsection{NASP Ramp Panel}

Culler and McNamara [101] computed the coupled fluid-thermal-structural response of a 3-D NASP-era ramp panel during a Mach 2 to 12 constant dynamic 


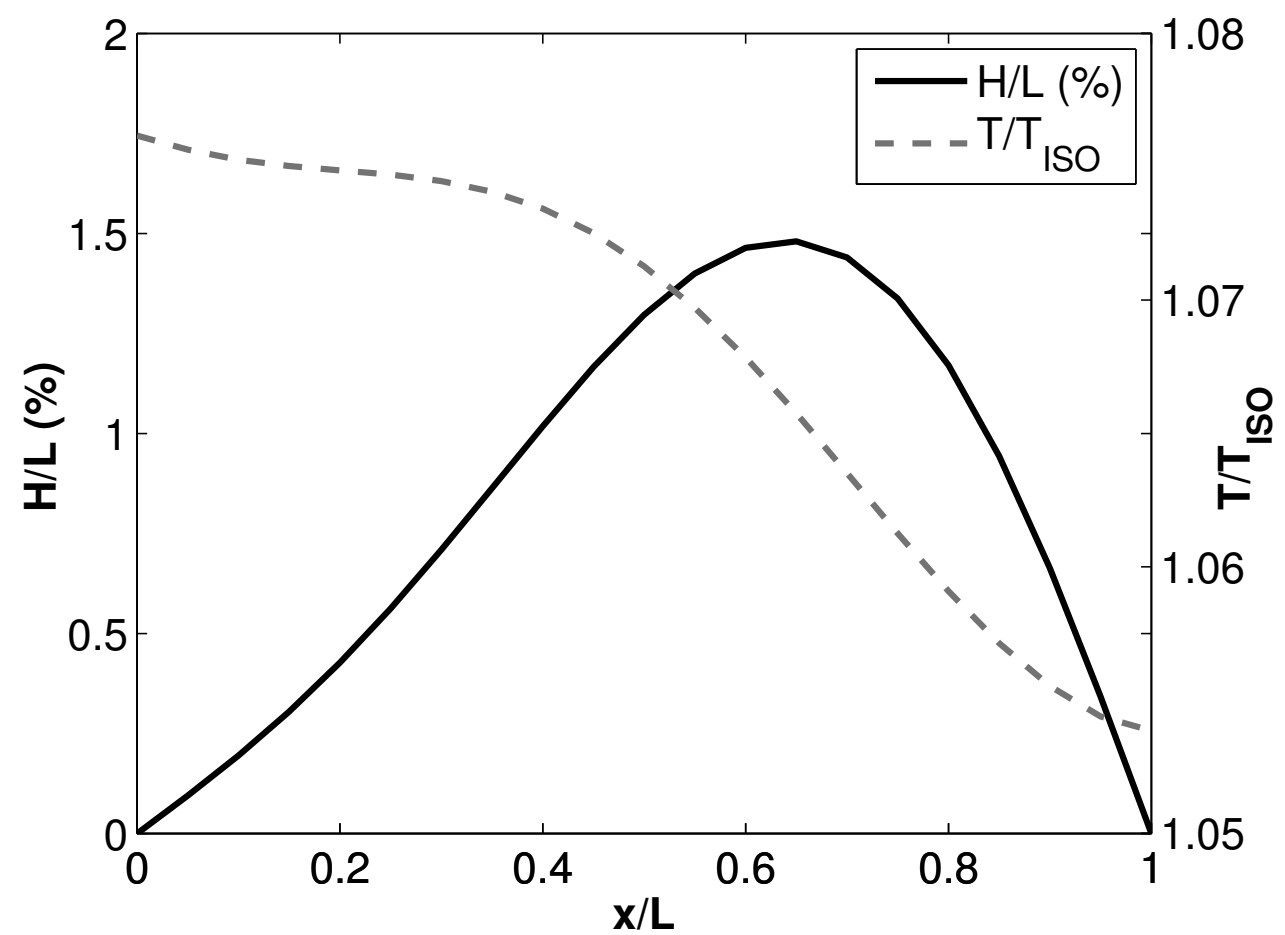

(a) Convex buckling.

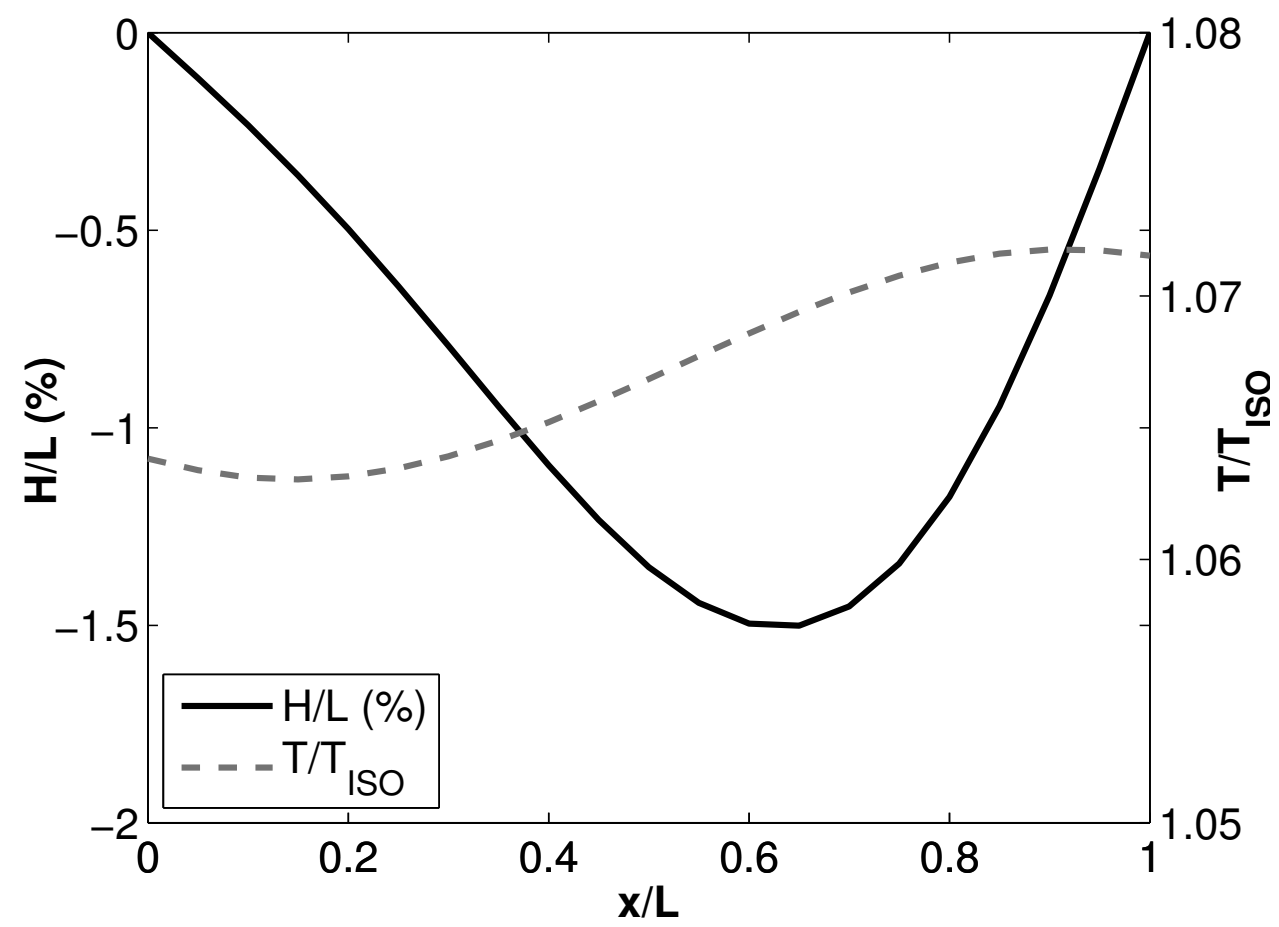

(b) Concave buckling.

Figure 3.1: Representative thermostructural responses extracted from [35] for a generic metallic panel. 
Table 3.1: Geometry and freestream conditions for generic metallic panel study.

\begin{tabular}{lc}
\hline \hline Parameter & Value \\
\hline Freestream Mach number & 4.00 \\
Freestream density, $\times 10^{-2} \mathrm{~kg} / \mathrm{m}^{3}$ & 1.79 \\
Freestream temperature, $\mathrm{K}$ & 231 \\
Effective altitude, $\mathrm{km}$ & 30.0 \\
Unit Reynolds number, $\times 10^{6} \mathrm{~m}^{-1}$ & 1.45 \\
Panel length, $\mathrm{m}$ & 1.00 \\
Wedge length, $\mathrm{m}$ & 10.0 \\
\hline \hline
\end{tabular}

pressure $(95.76 \mathrm{kPa})$ ascent trajectory. The 3-D panel studied in [101] is shown in Fig. 3.2. In this work, 2-D centerline displacements are extracted from the Mach 2 to 12 trajectory to observe the effect of combined Mach number and altitude variation, panel mode shape and surface temperature. The extracted displacements and corresponding times into the trajectory are shown in Fig. 3.3.a, where higher mode deformations appear beyond 240 seconds. The ascent trajectory from 90 to $300 \mathrm{~s}$ is shown in Fig. 3.3.b, where the legend provides the Mach and Reynolds numbers associated with the different operational times. Note that as the vehicle ascends, the Mach number increases and the Reynolds number decreases.

The wedge geometry used to model the ramp panel ascent trajectory is detailed in Table 3.2. The freestream conditions, corresponding to the time steps in Fig. 3.3.a are listed in Table 3.3. For this case, temperature profiles along the panel are not extracted from [101], since specifying the wall temperature as described in Section 3.1.1 would result in severe changes in the wall temperature between the undeformed and deformed sections of the wedge. Thus, in order to avoid carrying 


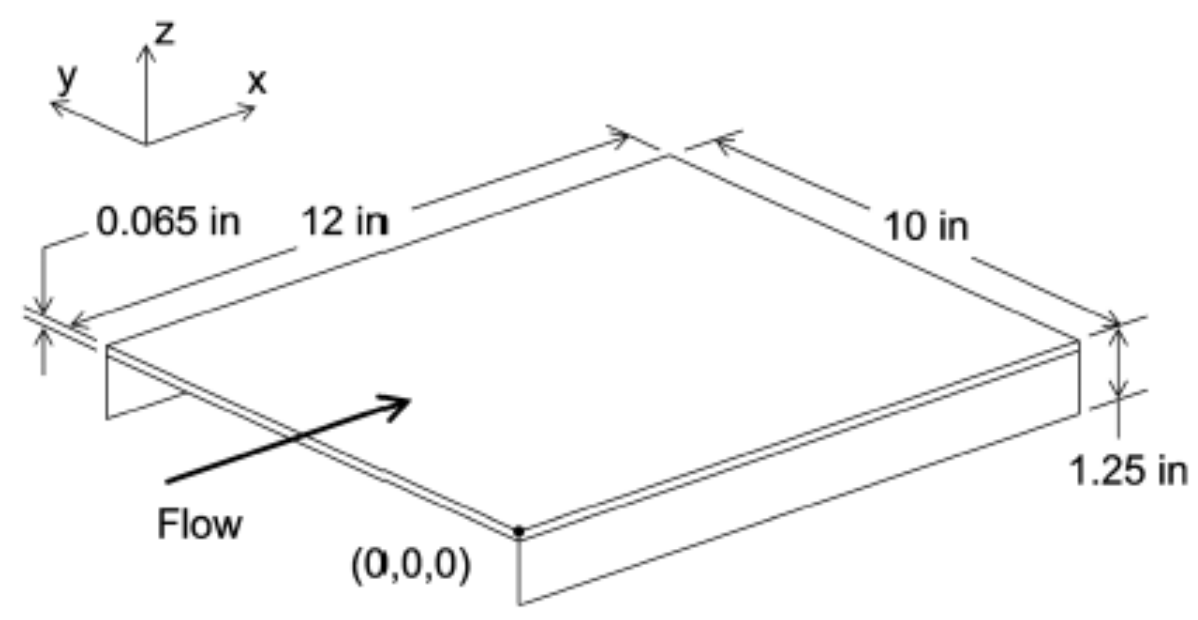

Figure 3.2: NASP-era ramp panel (figure 3 of [101]).

out a transient thermal response analysis of the entire wedge surface, the limiting cases of a cold wall $(294 \mathrm{~K})$ and radiative equilibrium wall are specified for the entire wedge surface.

Table 3.2: Geometry for NASP ramp panel ascent trajectory.

\begin{tabular}{lc}
\hline \hline Parameter & Value \\
\hline Panel leading edge location, $\mathrm{m}$ & 1.70 \\
Panel length, $\mathrm{cm}$ & 30.5 \\
Wedge length, $\mathrm{m}$ & 3.60 \\
\hline \hline
\end{tabular}

Table 3.3: Flow conditions for NASP ramp panel ascent trajectory.

\begin{tabular}{cccccc}
\hline $\begin{array}{c}\text { Time, } \\
\mathrm{s}\end{array}$ & $\begin{array}{c}\text { Mach } \\
\text { Number }\end{array}$ & $\begin{array}{c}\text { Density, } \\
\times 10^{-2} \mathrm{~kg} / \mathrm{m}^{3}\end{array}$ & $\begin{array}{c}\text { Temperature, } \\
\mathrm{K}\end{array}$ & $\begin{array}{c}\text { Effective } \\
\text { Altitude, } \mathrm{km}\end{array}$ & $\begin{array}{c}R_{e_{\text {unit }},} \\
\times 10^{6} \mathrm{~m}^{-1}\end{array}$ \\
\hline 90 & 5 & 8.80 & 217 & 20.1 & 9.13 \\
180 & 8 & 3.35 & 223 & 26.2 & 5.50 \\
210 & 9 & 2.63 & 224 & 27.7 & 4.84 \\
240 & 10 & 2.11 & 226 & 29.1 & 4.32 \\
300 & 12 & 1.45 & 228 & 31.6 & 3.55 \\
\hline \hline
\end{tabular}




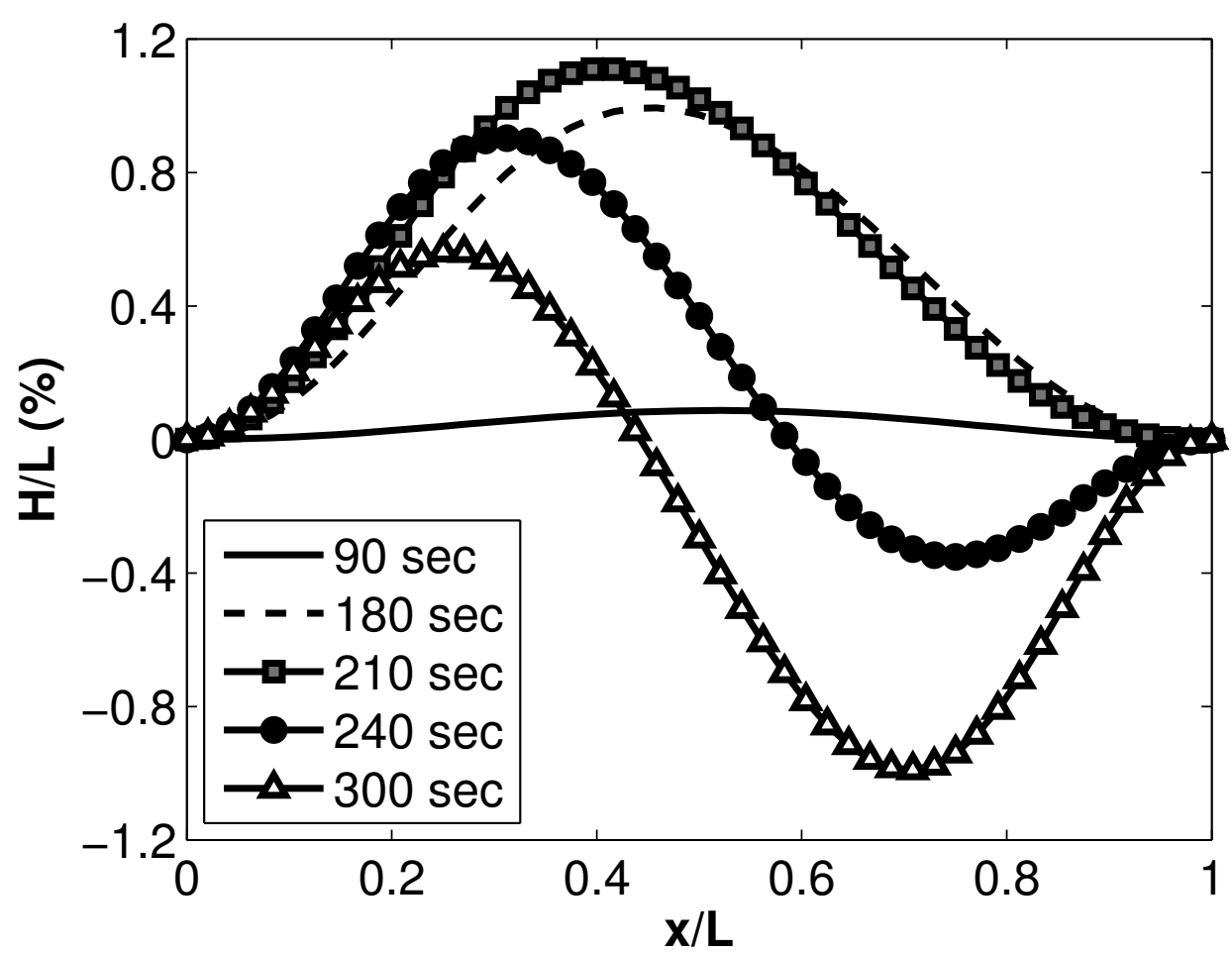

(a) Centerline displacements.

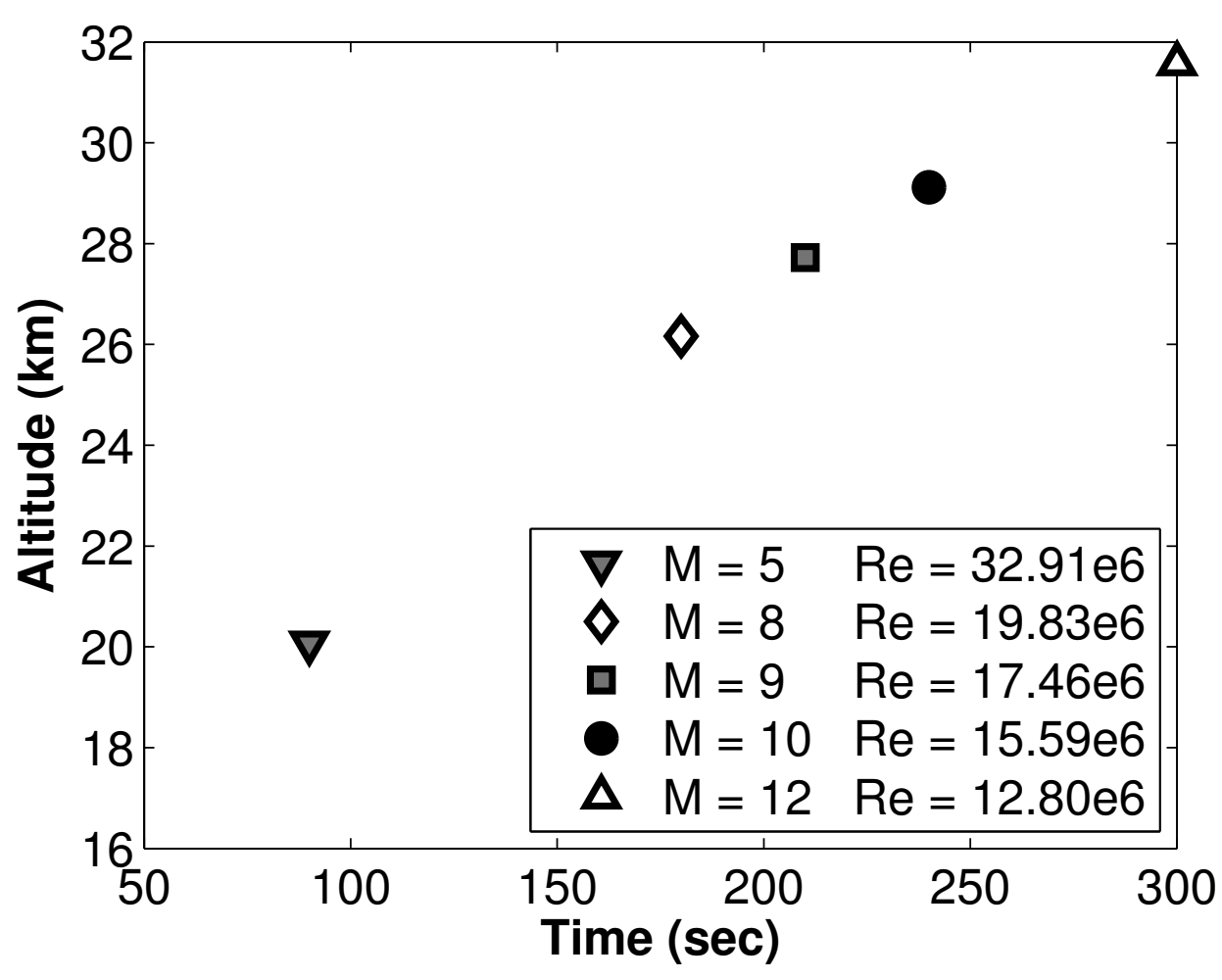

(b) Ascent trajectory.

Figure 3.3: Representative responses of NASP ramp panel. 


\subsubsection{Spherical Domes}

Glass and Hunt [93] experimentally characterized aerothermal load augmentation on spherical domes, representative of bowed surface panels, in the Langley $8 \mathrm{ft}$. High-Temperature Tunnel. The experimental set up of [93] is depicted in Fig. 3.4, where it can be seen that domes of various length and leading edge location were examined. In this study, 2-D deformations are developed from the dome geometries listed in Table 3.4 to observe the impact of panel size, displacement into the flowfield and structural boundary condition on boundary-layer stability. Note that domes 1-3 and 4-6 have the same length and leading edge location but different peak displacements. The dome length listed in Table 3.4 corresponds to the dome diameter values in [93]. As shown in Fig. 3.5, both "clamped" and spherical or "simply-supported" deformations are considered here to examine the effect of leading and trailing edge slope. Also, consideration of these boundary conditions provides a means to encompass the anticipated effect of an actual structural panel on stability as its attachment to the vehicle body will resemble something between these idealistic cases.

Table 3.4: Spherical dome dimensions [93].

\begin{tabular}{cccc}
\hline \hline Dome \# & Length, $\mathrm{cm}$ & Height, $\mathrm{mm}$ & Leading edge, $\mathrm{m}$ \\
\hline 1 & 17.78 & 1.262 & 1.887 \\
2 & 17.78 & 2.525 & 1.887 \\
3 & 17.78 & 5.050 & 1.887 \\
4 & 35.56 & 2.542 & 1.798 \\
5 & 35.56 & 5.085 & 1.798 \\
6 & 35.56 & 10.17 & 1.798 \\
\hline \hline
\end{tabular}




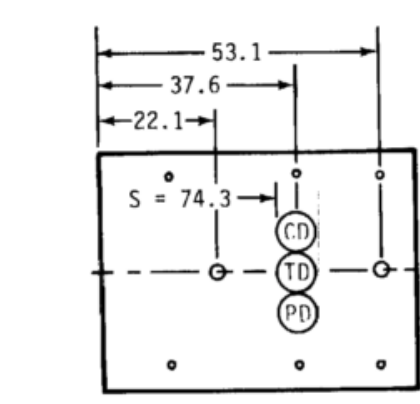

Panel insert of 7-in-diameter dome

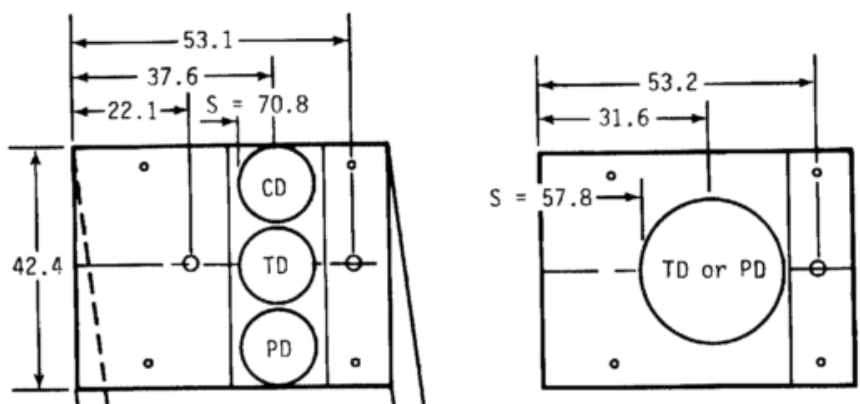

Panel insert of 28-in-diameter dome

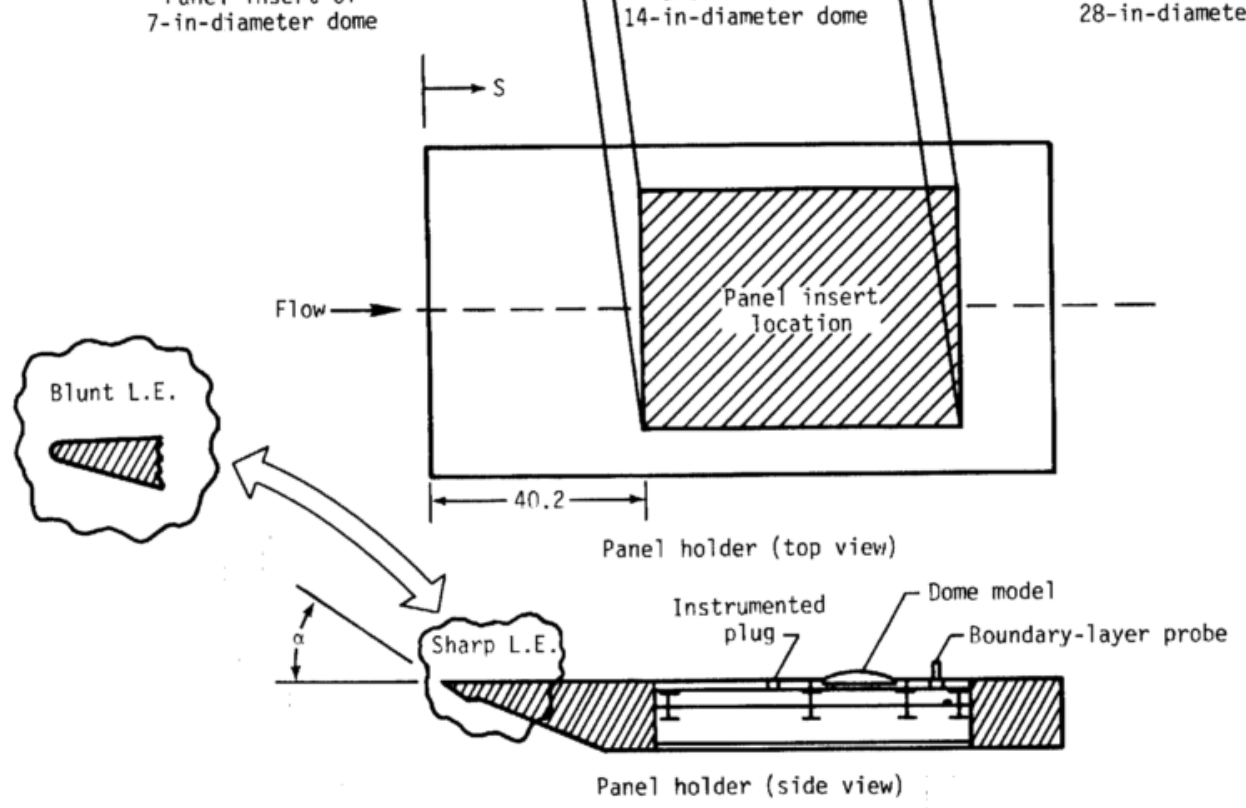

Figure 3.4: Schematic of Glass and Hunt experimental geometry with dimensions in inches (figure 2 of [93]). 


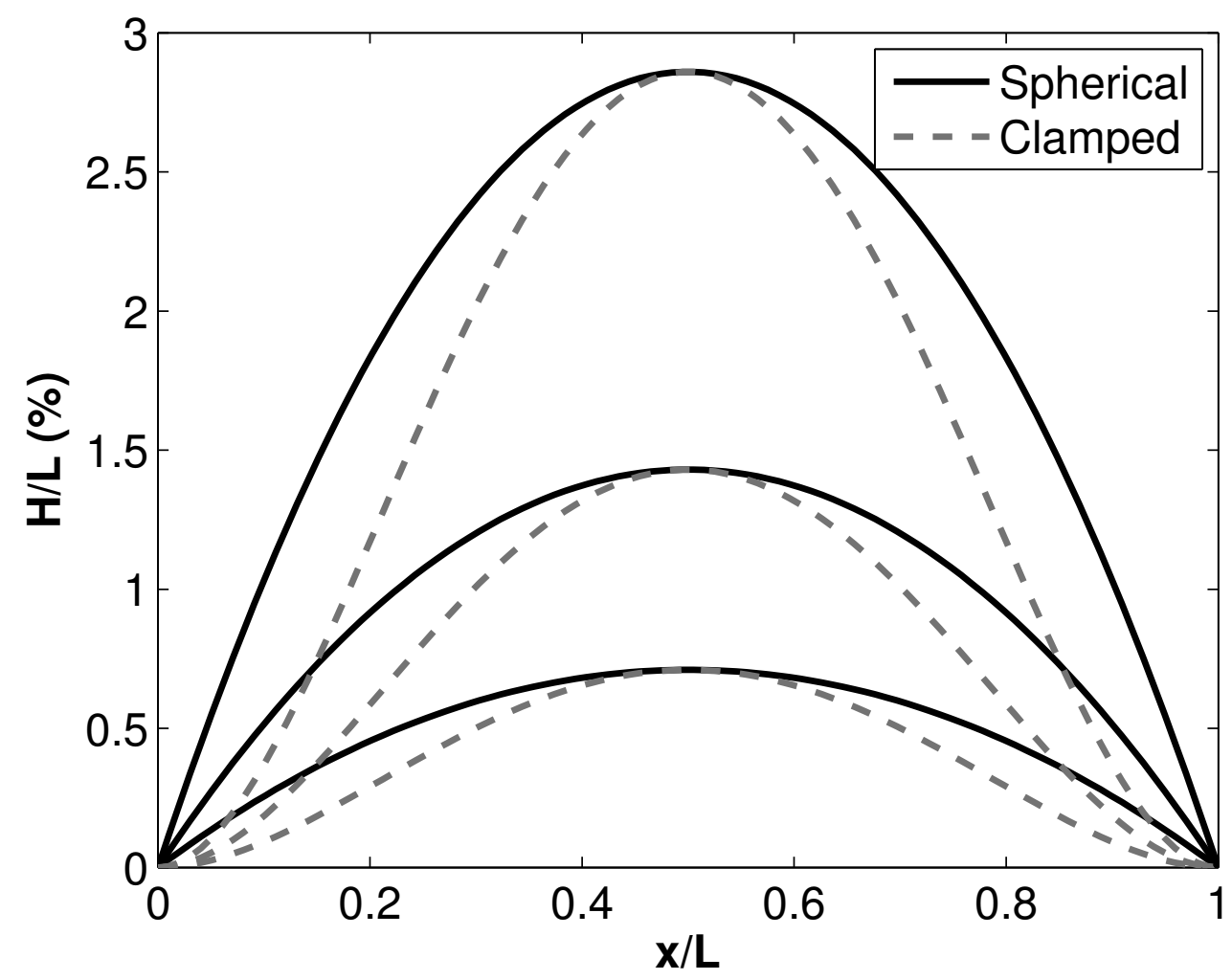

Figure 3.5: Displacement profiles for spherical and clamped dome configurations. 
A complete description of the model geometry and freestream conditions is given in Table 3.5. The freestream conditions are obtained through static flow properties. The wedge length matches the experimental setup of Glass and Hunt [149]. Again, the limiting cases of a cold wall (300 K) and radiative equilibrium wall temperature are considered for the entire wedge surface temperature.

Table 3.5: Geometry and flow conditions for spherical domes.

\begin{tabular}{lc}
\hline \hline Parameter & Value \\
\hline Freestream Mach number & 6.5 \\
Freestream density, $\times 10^{-2} \mathrm{~kg} / \mathrm{m}^{3}$ & 1.80 \\
Freestream temperature, $\mathrm{K}$ & 227 \\
Effective altitude, $\mathrm{km}$ & 30.2 \\
Unit Reynolds number, $\times 10^{6} \mathrm{~m}^{-1}$ & 1.93 \\
Wedge length, $\mathrm{m}$ & 3.00 \\
\hline \hline
\end{tabular}

\subsubsection{Nondimensional Panel Comparison}

A nondimensional characterization of each deformation is provided in Table 3.6 in terms of the deformation height $(H)$, length $(L)$ and boundary-layer thickness $(\delta)$. The boundary-layer thickness is defined as the wall-normal location where the enthalpy achieves $99.5 \%$ of the freestream total condition. The $H / \delta$ values for the Generic Metallic Panel and Spherical Dome deformations exceed that of past work focused on roughness-scale deformations [48, 49,51]. However, the $L / \delta$ range for each study indicates that the deformation lengths are orders of magnitude larger than the boundary-layer thickness. Therefore, these deformations result in slowly 
varying, large-scale surface curvatures. Furthermore, the surface curvature of the deformations is significantly smaller than that of several blunt cones [150,151] that have previously been examined with the LPSE [120, 152-154]. Also, the length of the deformations in Table 3.6 are in general an order of magnitude larger than the wavelengths of the instabilities present over the deformation location.

Several of the deformations described in Table 3.6 result in small regions of flow separation near the leading and trailing edges. As separation produces regions of recirculating flow, in which information propagates upstream, the applicability of LPSE comes into question. However, work by Theofilis et al. [155] has shown, through agreement between LPSE and DNS, that upstream influences within a separated region are negligible if the dominant disturbances are convective.

Table 3.6: Nondimensional deformation comparison.

\begin{tabular}{lccc}
\hline \hline Study & $H / \delta$ & $L / \delta$ & $H / L(\%)$ \\
\hline Generic Metallic Panel & $1.13-2.56$ & $76-173$ & 1.48 \\
NASP Ramp Panel & $0.09-0.66$ & $43-100$ & $0.09-1.11$ \\
Spherical Domes & $0.20-1.58$ & $28-55$ & $0.71-2.86$ \\
\hline \hline
\end{tabular}

\subsection{Mean Flow Grid Convergence}

A grid resolution study is performed to determine the required mean flow mesh density. The velocity and temperature derivatives for the most severe deformation in Fig. 3.6 demonstrate grid independence of the boundary layer with 400 surfacenormal grid points. The resolved grids have a maximum cell spacing along the 
surface of $10 \mathrm{~mm}$, where hyperbolic cell spacing is used to cluster cells near the leading and trailing edge of each deformed section of the surface geometry. A maximum cell spacing of $5 \mathrm{~mm}$ is used over the deformed section of the grid. For the resolved grids, 100 cells define the boundary layer and a $y^{+}$value of less than 0.8 is achieved in the first cell from the surface over the entire geometry. Grid independence of the stability analysis is demonstrated with respect to the mean flow mesh density (Fig. 3.7.a) and the LPSE test matrix density (Fig. 3.7.b).

\subsection{Results}

The results are divided according to the applied panel geometry. In Sections 3.3.13.3.2 disturbance amplification contours, obtained through LST, are provided to highlight shifts in the unstable frequencies caused by deformation. Note that the unstable frequencies predicted using LST are in general agreement with those obtained using the LPSE. In addition, the boundary-layer thickness over the wedge is examined to study the effect of different deformation shapes on the mean flow. Also, maximum $N$ factor envelopes are compared to quantify the influence of 2-D deformations on boundary-layer stability. Finally, for each case the 2-D highermode instabilities are found to be more dominant than the oblique first mode. Therefore, the following results pertain to 2-D, streamwise oriented disturbances. 


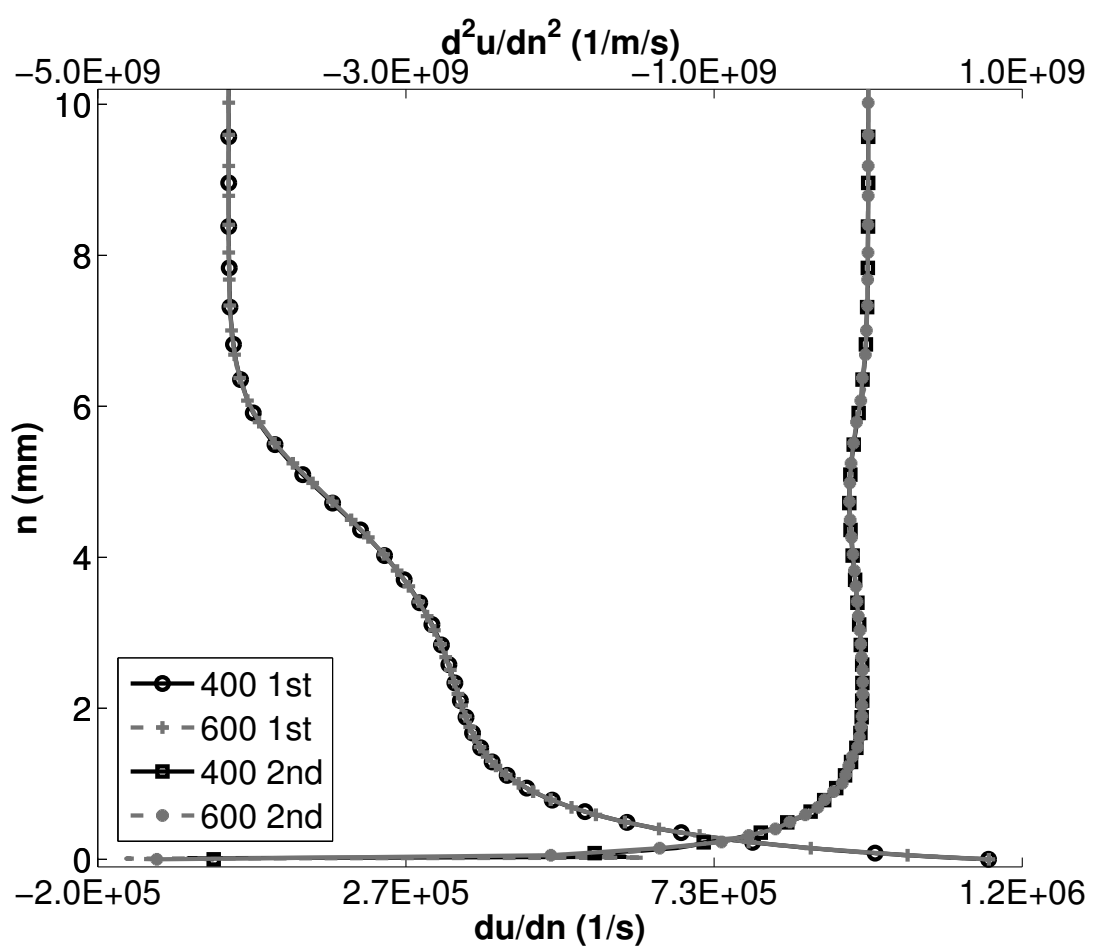

(a) Velocity.

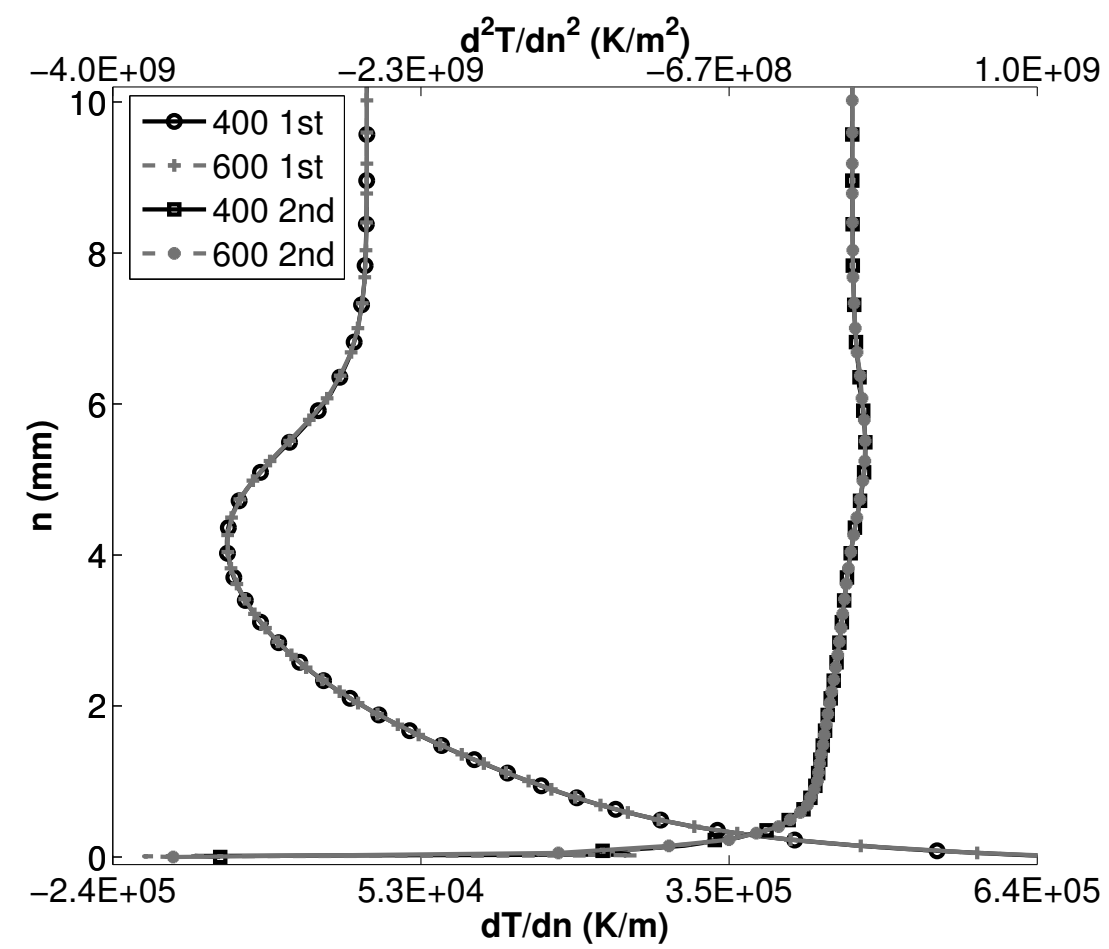

(b) Temperature.

Figure 3.6: Wall-normal derivatives at peak deformation location (Glass and Hunt dome 6 [93]). The legend indicates the number of wall normal grid points and either $1^{\text {st }}$ or $2^{\text {nd }}$ derivative. 


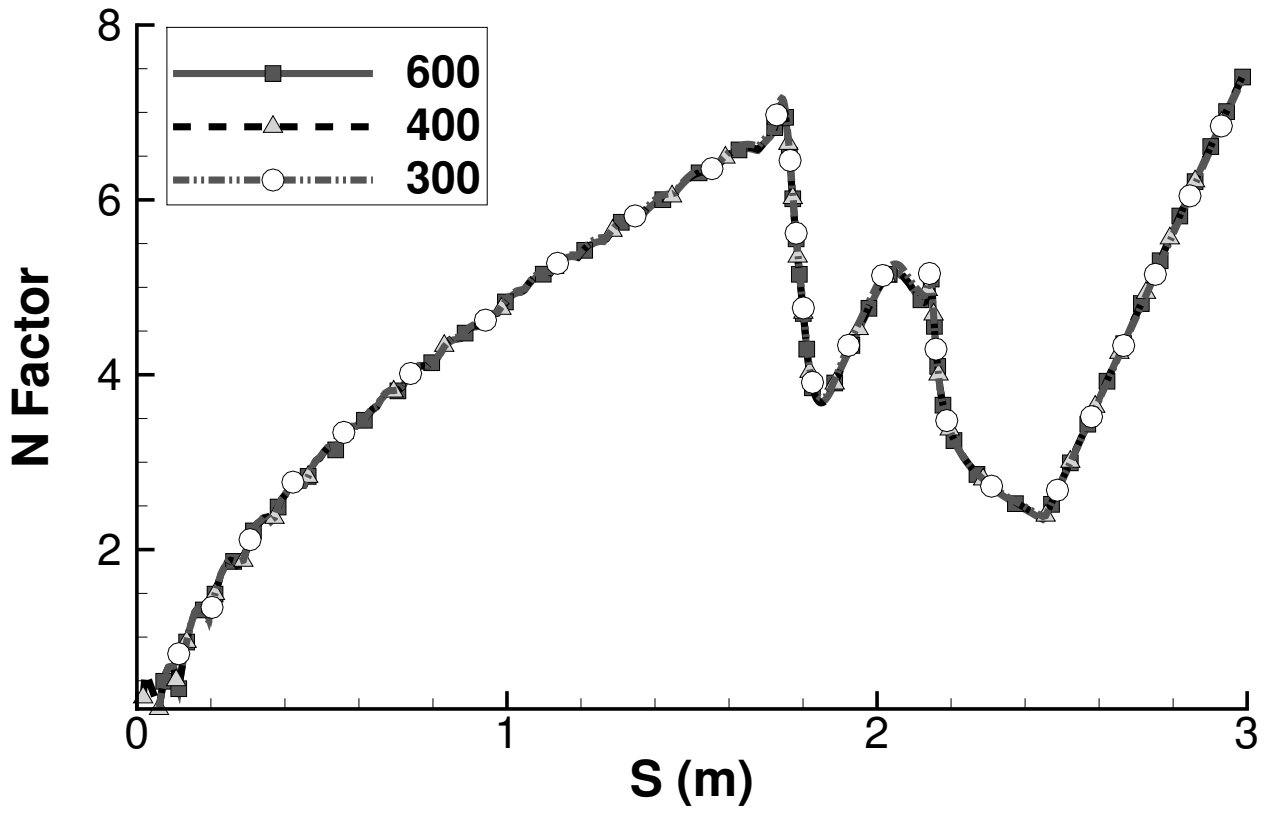

(a) Varying wall-normal mean flow grid points.

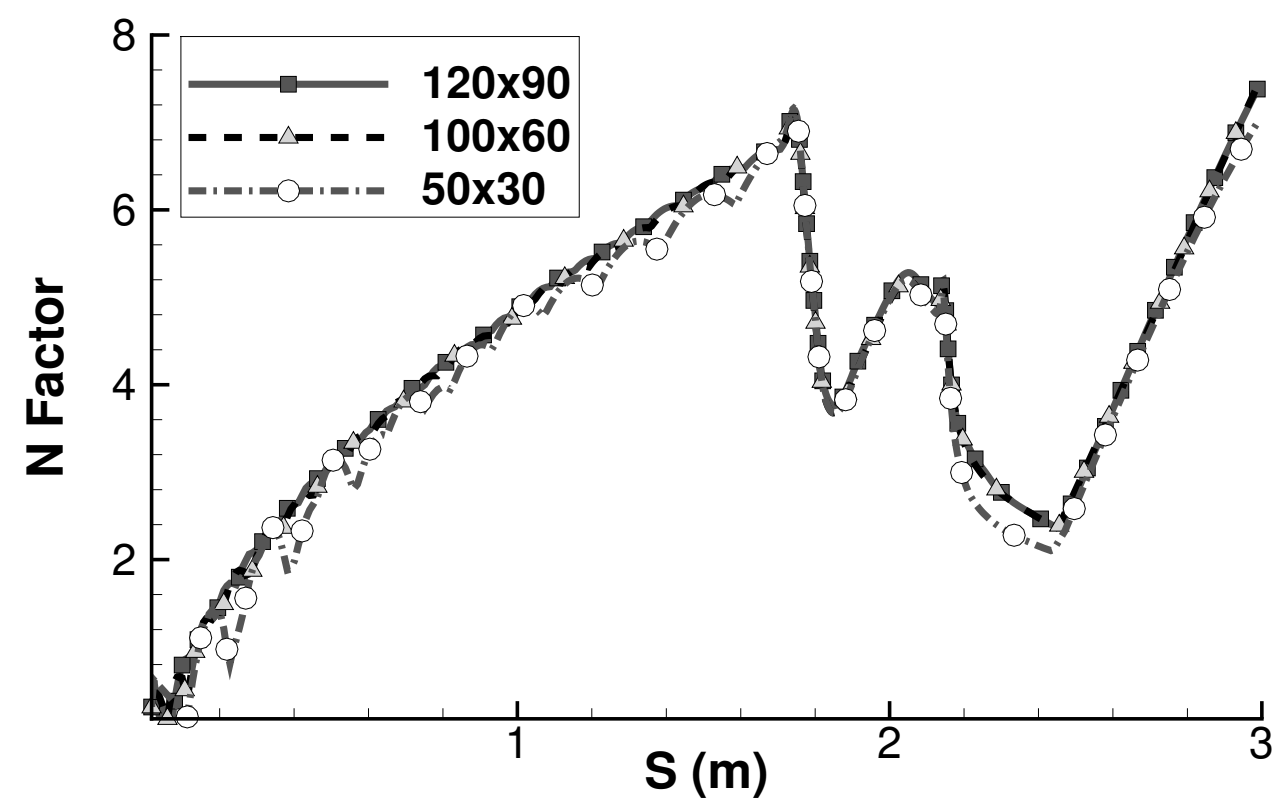

(b) Varying LPSE test matrix (distance $\times$ frequency) density.

Figure 3.7: LPSE resolution study for the maximum deformation case (Glass and Hunt dome 6 [93]). 


\subsubsection{Generic Metallic Panel}

The deformation and temperature profiles shown in Fig. 3.1 are used to analyze how deformation direction, panel location and multiple panel arrangements affect flow stability. For the panel location study in Section 3.3.1.1, the deformation is placed at 1,3,5, and $7 \mathrm{~m}$ from the leading edge of the wedge. For the multiple panel study in Section 3.3.1.2, up to four panels in series are examined.

\subsubsection{Panel Location}

Disturbance amplification contours corresponding to several panel locations are shown in Fig. 3.8, where the amplification rate $\left(-\alpha_{i}\right)$ contours greater than zero indicates unstable disturbance growth. The convex cases in Fig. 3.8 demonstrate that at each location the panel produces the same relative change in stability, where the forward portion of the deformation shifts the unstable disturbances to higher frequencies and the aft section results in a shift to lower frequencies. The inverse of this trend is true for the concave deformations. This frequency shift is associated with the manipulation of the boundary layer by the deformation, as shown in Fig. 3.9. The vertical bar in Fig. 3.9 signifies the panel location, which is also indicated by the scaled representation of the wedge surface labeled as "Wedge". For convex buckling in Fig. 3.9.a, the boundary-layer thickness on the forward compression side of the panel is less than that of the rigid wedge, while on the expansion side, beginning at $1.7 \mathrm{~m}$, the boundary-layer thickness is greater. For 
the concave panel in Fig. 3.9.b, this trend is reversed as the flow goes through expansion then compression. The results in Fig. 3.8 are consistent with Marxen et al. [49] which found that roughness-scale protuberances can amplify or damp instabilities depending on the frequency range. An additional item to note from Fig. 3.8 is that surface compliance could have a strong impact on boundary-layer stability by shifting unstable disturbances to different frequencies. This disrupts the instability band in the LST diagram, reducing the amplification and distance along the surface that specific frequencies are unstable. Furthermore, the panel location could play a strong role in how stability is affected. Near the leading edge, where the most unstable disturbance frequency is rapidly decreasing, inclusion of the panel increases both the amplification and distance of unstable growth for a given frequency. However, when the panel is placed further downstream, where unstable growth occurs over longer distances, the effect is to shift instabilities to higher and lower frequencies. This effectively introduces stable regions for what would be unstable growth of certain disturbance frequencies in the absence of surface compliance.

For the LPSE results in Fig. 3.10 the shaded vertical and horizontal bars designate the panel location and expected transitional $N$ factor range. Fig. 3.10.a indicates that inclusion of the panels at $1 \mathrm{~m}$, increases the $N$ factors immediately downstream of the panel, with a recovery to the rigid wedge maximum $N$ factor envelope beginning approximately at $5 \mathrm{~m}$. This is a result of the panel at $1 \mathrm{~m}$ exciting high frequency disturbances near $50 \mathrm{kHz}$ and $100 \mathrm{kHz}$ that become stable 


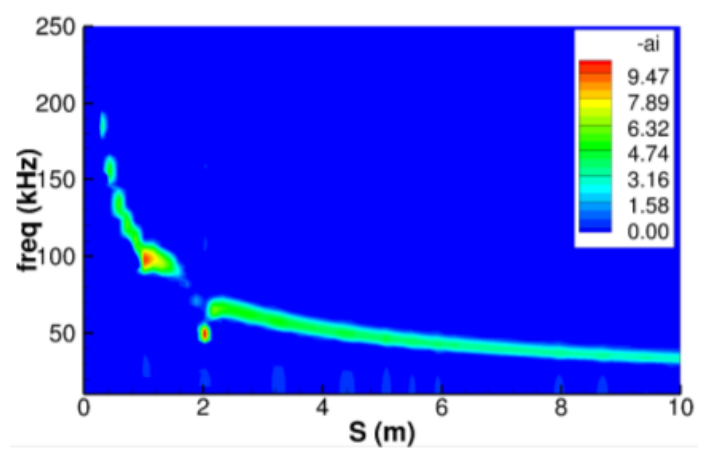

(a) Convex panel response at $1 \mathrm{~m}$.

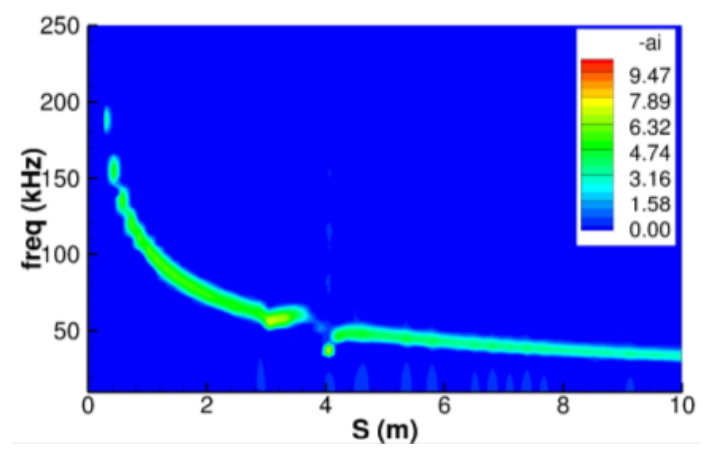

(c) Convex panel response at $3 \mathrm{~m}$.

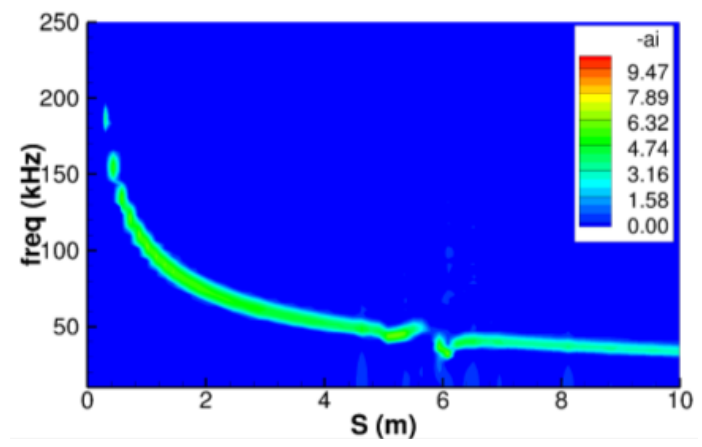

(e) Convex panel response at $5 \mathrm{~m}$.

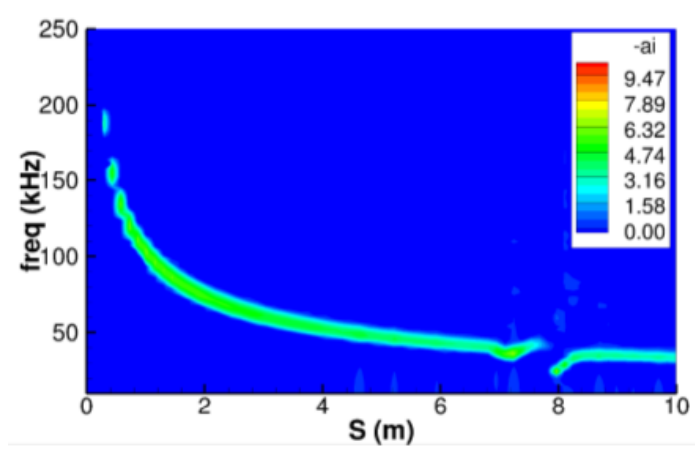

(g) Convex panel response at $7 \mathrm{~m}$.

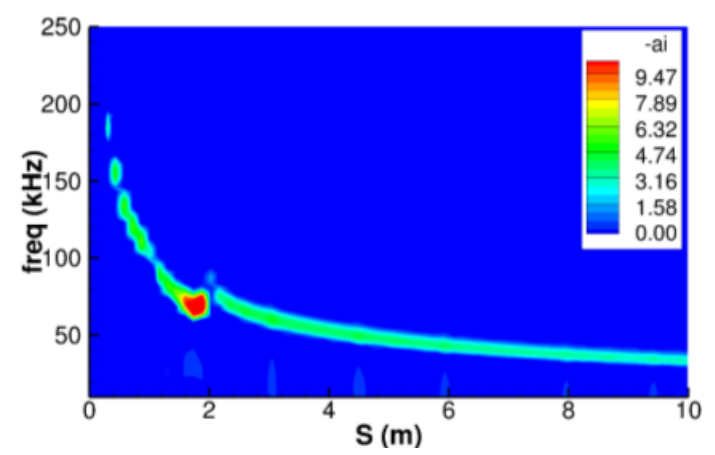

(b) Concave panel response at $1 \mathrm{~m}$.

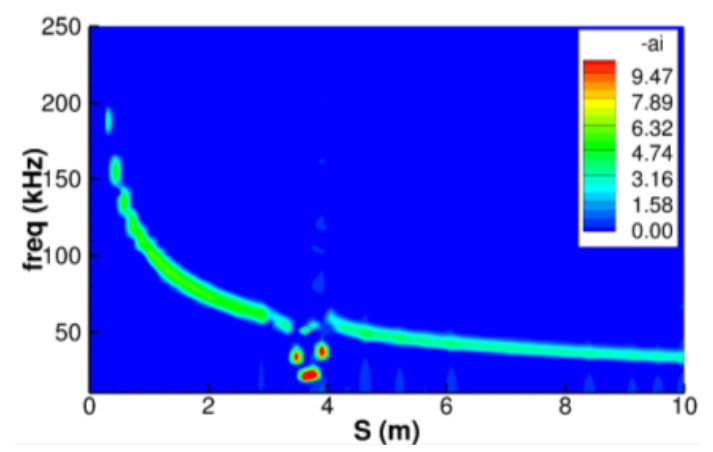

(d) Concave panel response at $3 \mathrm{~m}$.

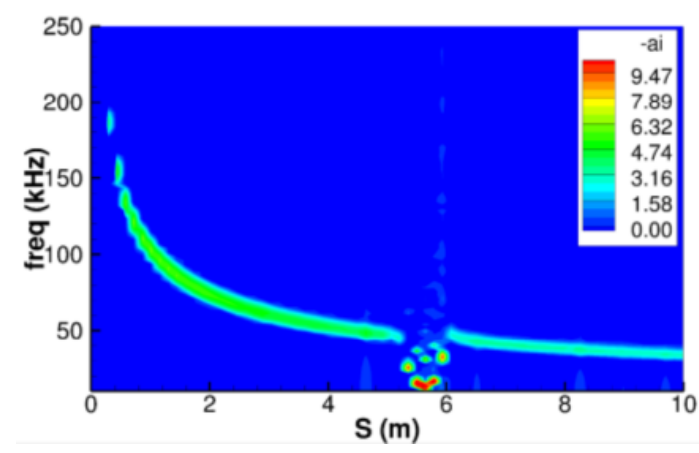

(f) Concave panel response at $5 \mathrm{~m}$.

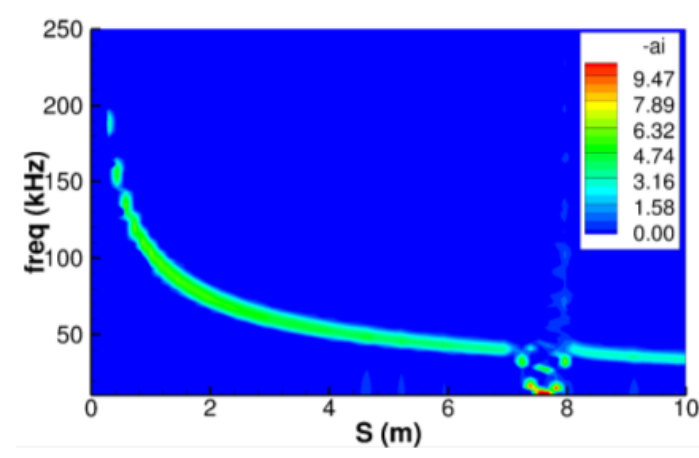

(h) Concave panel response at $7 \mathrm{~m}$.

Figure 3.8: Stability diagrams for different panel locations. 


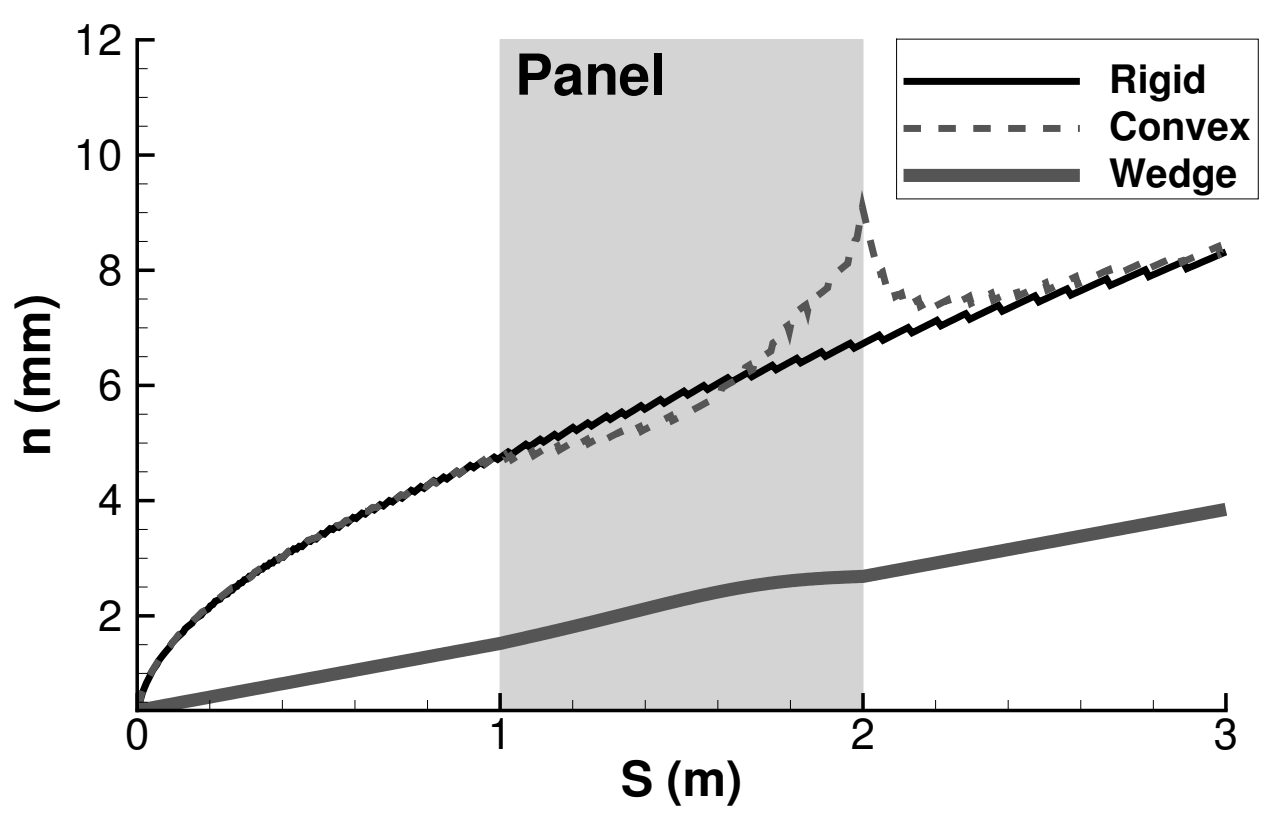

(a) Convex.

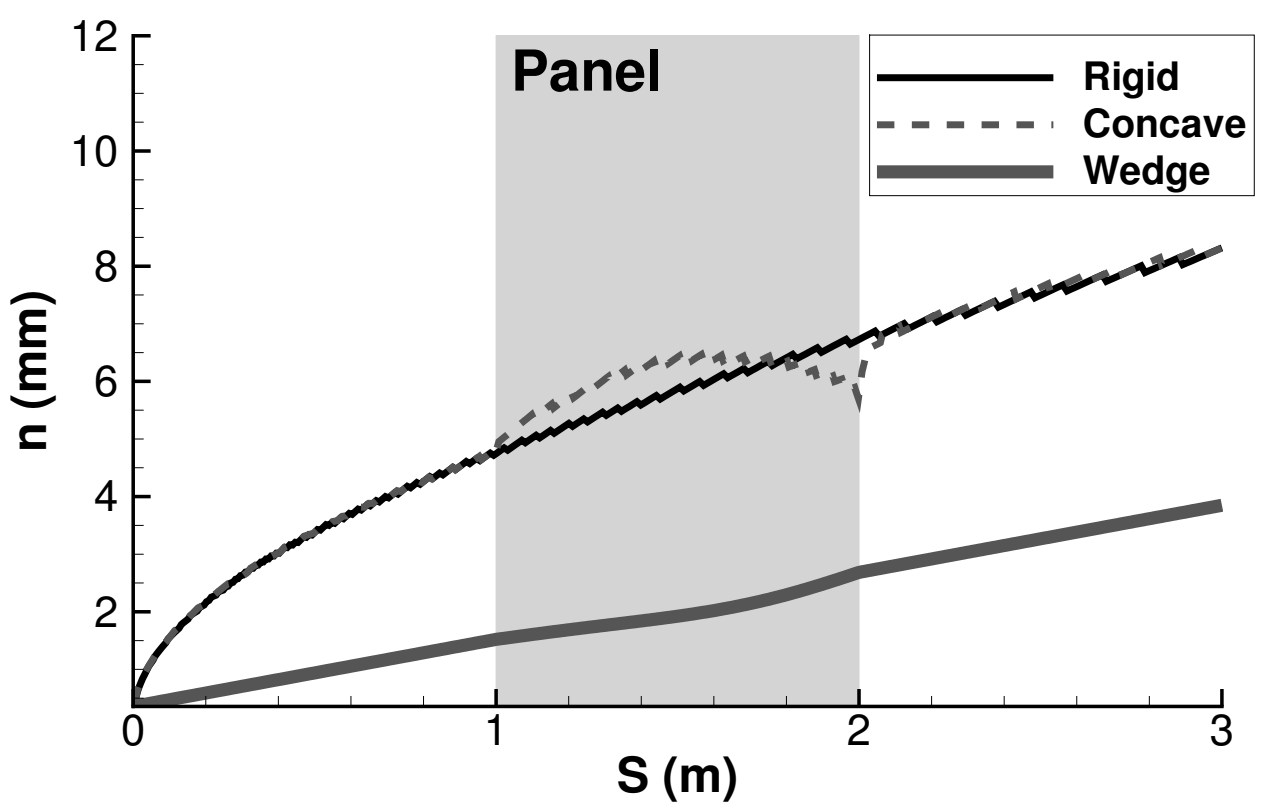

(b) Concave.

Figure 3.9: Boundary-layer thickness for rigid wedge and panel response at $1 \mathrm{~m}$. 
by $5 \mathrm{~m}$ due to the boundary-layer growth. When the panel is placed at $3 \mathrm{~m}$, there is an initial decrease in the $N$ factors, followed by different amounts of growth for the convex and concave panels. The convex panel causes the $N$ factor curve to rise more rapidly into the transitional range than the rigid wedge, while the $N$ factor curve for the concave panel remains lower until the end of the wedge. As the panel is moved aft of $3 \mathrm{~m}$, the effect is similar in that there is a drop in the $N$ factors aft of the panel, followed by a rise. However, as the panel is moved towards the trailing edge, there is less distance for the disturbances to grow.

Further examination of Fig. 3.10 reveals that, for the convex panel located at

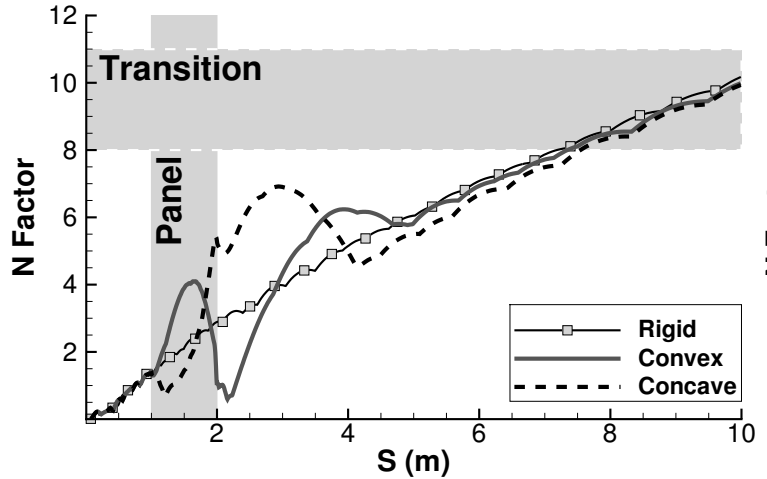

(a) Panel response at $1 \mathrm{~m}$.

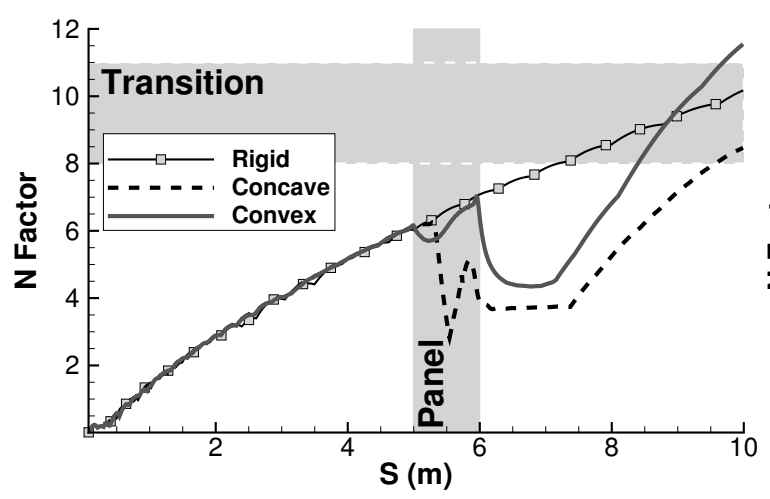

(c) Panel response at $5 \mathrm{~m}$.

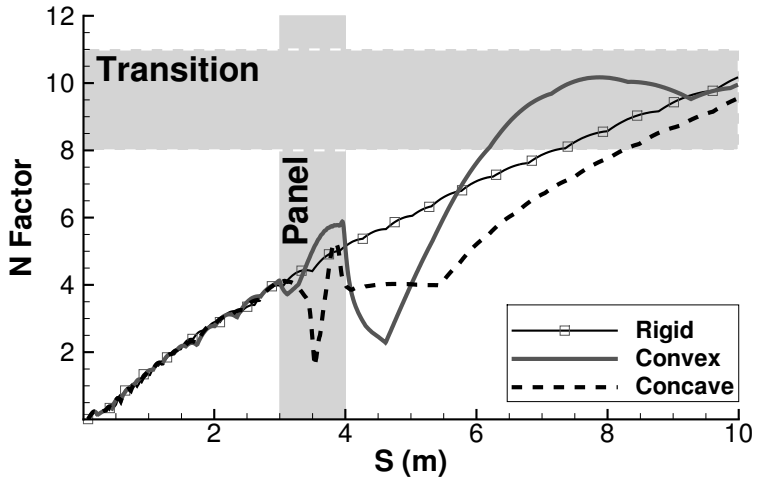

(b) Panel response at $3 \mathrm{~m}$.

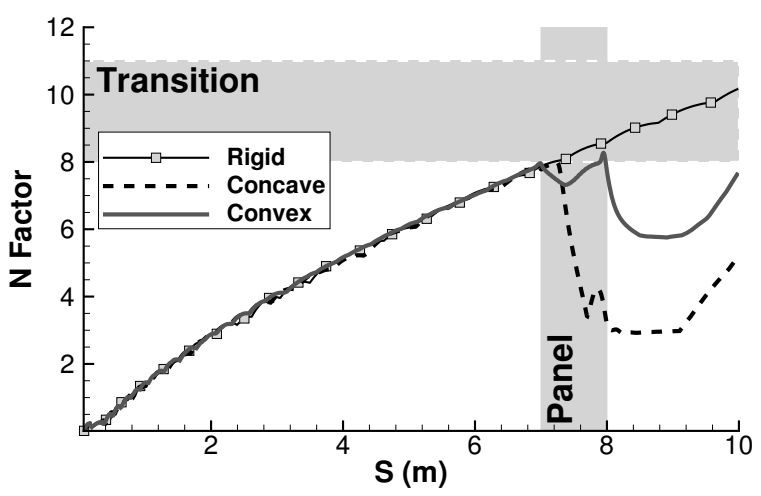

(d) Panel response at $7 \mathrm{~m}$.

Figure 3.10: Maximum $N$ factor comparison for different panel response locations. 
$3 \mathrm{~m}$, the intersection of the $N$ factor curve and the transitional region has moved forward on the body as compared with the rigid wedge results. However, for panel locations beyond $3 \mathrm{~m}$ inclusion of the convex panel moves the expected transition point backward on the body. This finding is consistent with the work of Duan et al. [51] which has recently shown that 2-D roughness elements either destabilize or stabilize Mack mode disturbances when placed upstream or downstream of the synchronization point.

\subsubsection{Multiple Panels in Series}

From the previous results, it is clear that the deformations generally have a positive influence on boundary-layer stability immediately following the panels. Thus, it is interesting to examine the impact of a series of compliant panels. The series panels begin $4 \mathrm{~m}$ from the leading edge of the wedge as the results in Section 3.3.1.1 suggest that panels placed further downstream, past the initial rapid boundary-layer growth, tended to have a positive impact on stability. To generate the CFD meshes, the panel responses in Fig. 3.1 are placed in series as shown in Fig. 3.11 where the temperature between panels is set to the average of the fore panel trailing edge and aft panel leading edge.

The LST results for configurations with up to four series panels are shown in Fig. 3.12. Examination of the stability diagrams in Figs. 3.12.a and 3.12.b indicate that both the convex and concave deformation of a single panel disrupts the relatively flattened section of the instability band. As additional panels are added in 


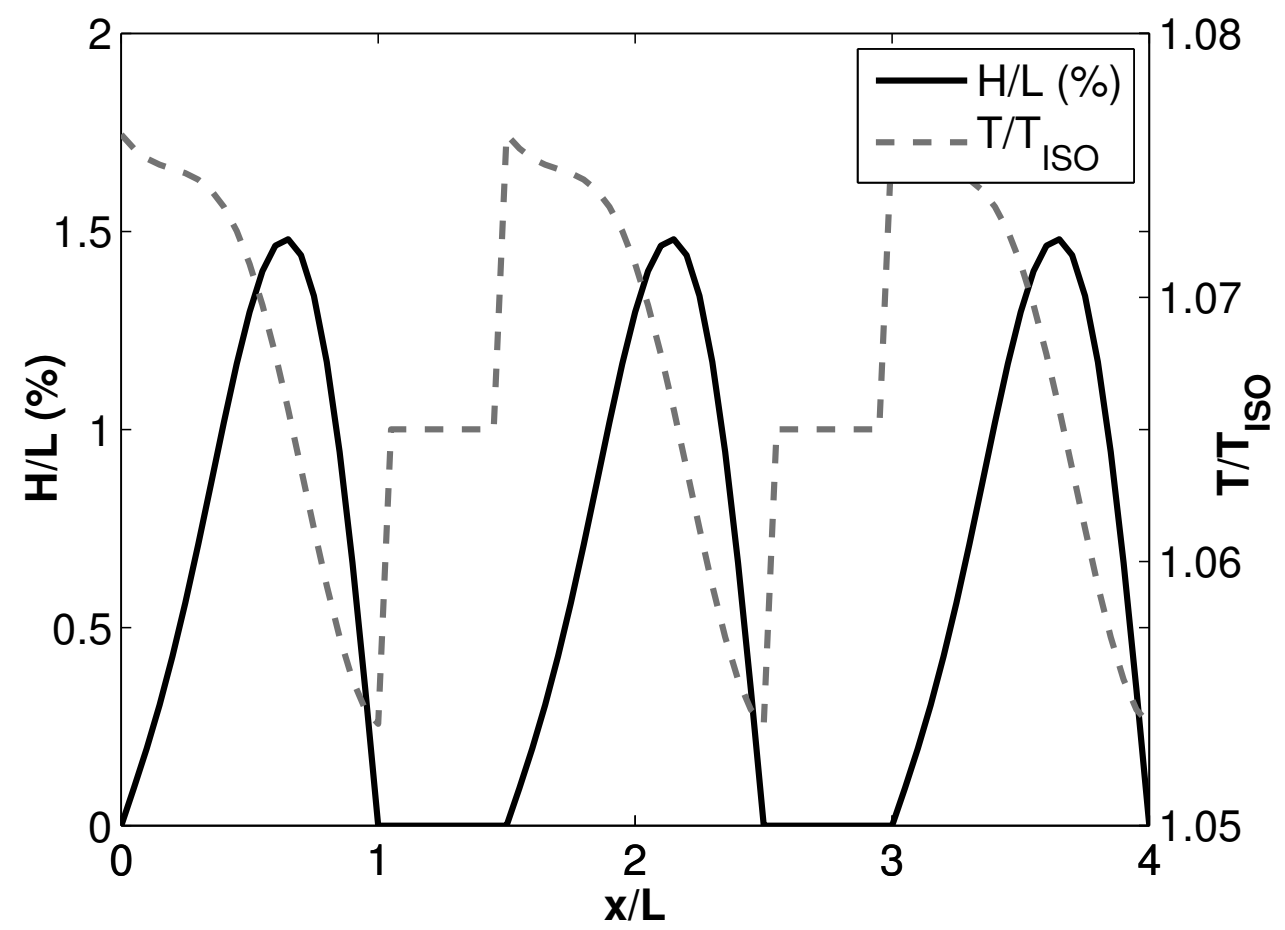

Figure 3.11: Configuration for three convex deformations in series.

Figs. 3.12.c-h this trend continues until the flattened portion of the instability band, which contains the disturbances that result in transition for the smooth wedge, is completely disrupted over the remainder of the wedge body. An interesting distinction between the convex and concave responses is that the latter comes at the cost of highly amplifying the same low frequency disturbances over each successive panel. Note that this finding is consistent with work by Bountin et al. [50], who found increased amplification at lower frequencies for hypersonic flow over a concave wavy wall.

The $N$ factor envelopes for the multiple panel configurations are shown in Fig 3.13. For the convex buckling cases in Fig. 3.13.a, including a single panel at 4 $\mathrm{m}$ is found to break up the monotonic growth of $N$ factors produced by the rigid 


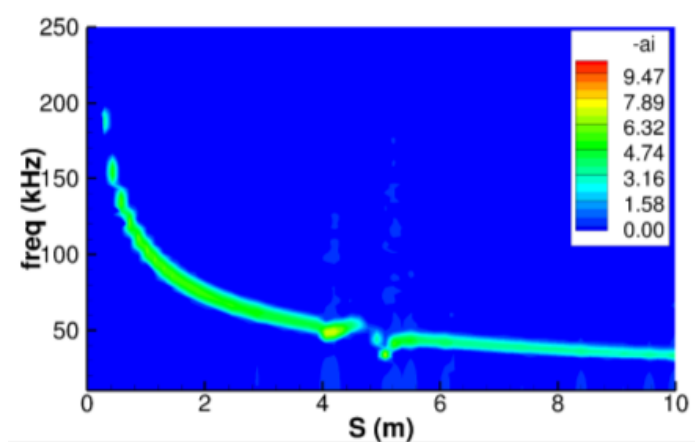

(a) One convex panels.

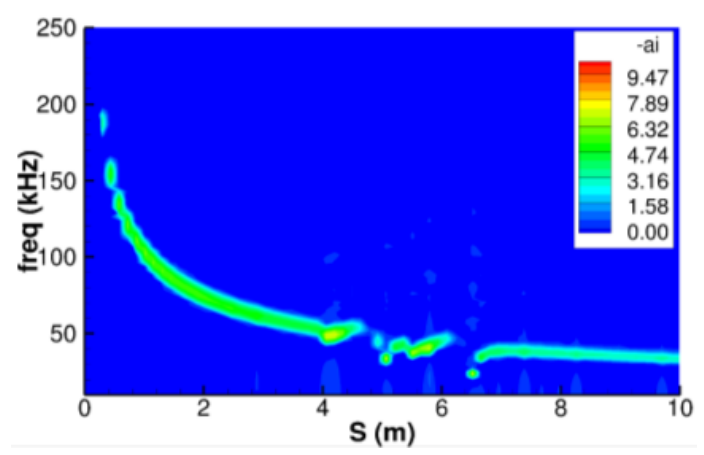

(c) Two convex panels.

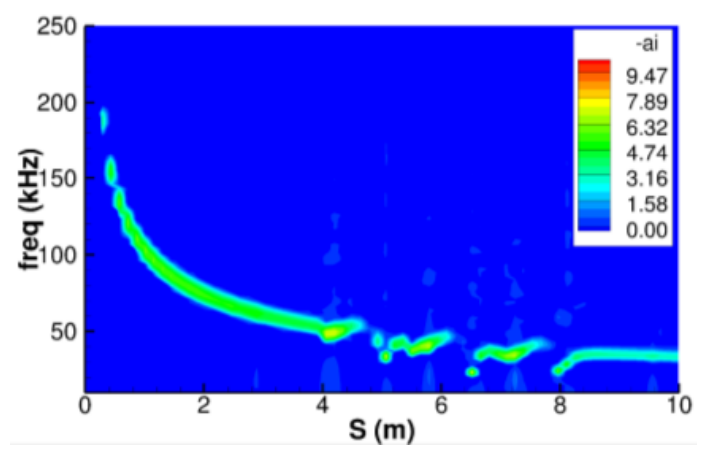

(e) Three convex panels.

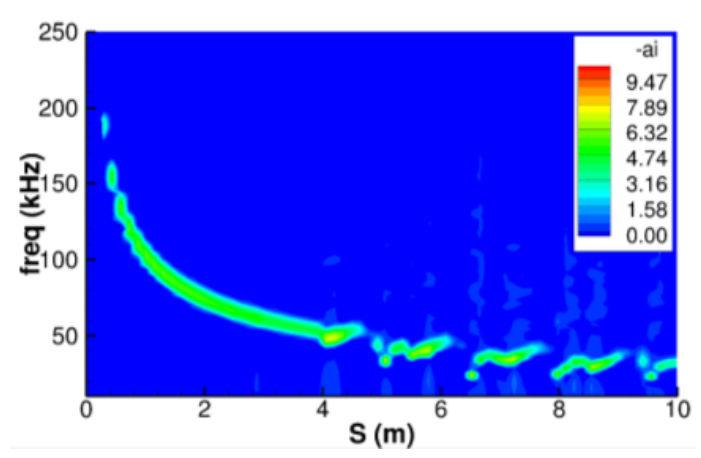

(g) Four convex panels.

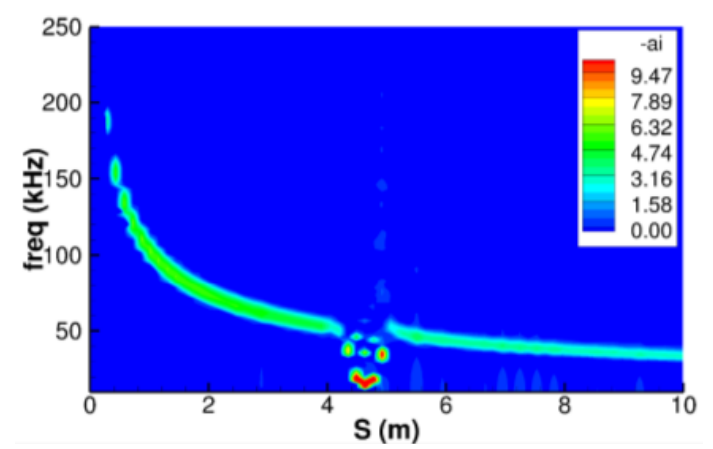

(b) One concave panels.

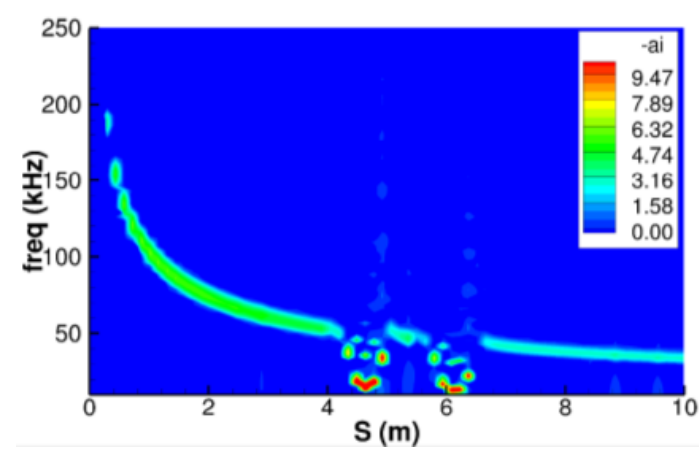

(d) Two concave panels.

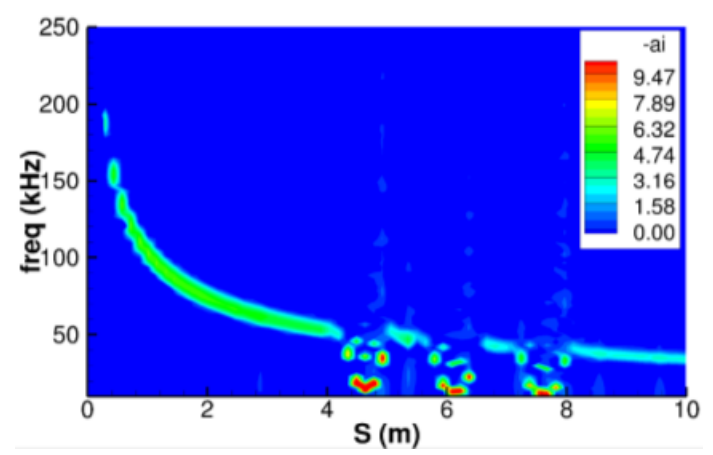

(f) Three concave panels.

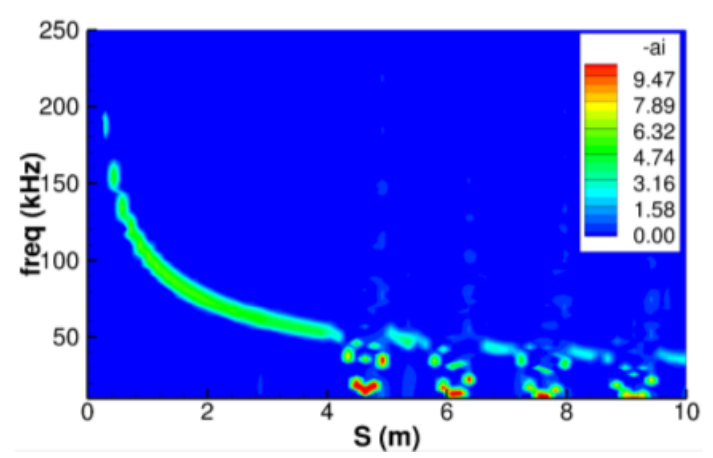

(h) Four concave panels.

Figure 3.12: Stability diagrams for multiple panels in series. 
wedge. Behind the panel there is a significant drop in $N$ factor from 5 to $6 \mathrm{~m}$, followed by an elevated $N$ factor growth rate that exceeds that of the rigid solution by $7.2 \mathrm{~m}$. However, as more panels are added, the maximum $N$ factor is continually decreasing, indicating a significant improvement in the boundary-layer stability over the aft, transition prone, regions of the wedge.

Boundary-layer stabilization has been demonstrated for micron-scale distributed roughness in subsonic [90] and supersonic [91] flow. More recently, multiple roughness elements or wavy wall roughness has been shown to delay transition [48] and stabilize Mack mode disturbances [50, 156] in hypersonic flows. A distinguishing feature of this study is that the deformation length is exceedingly larger than the boundary-layer thickness, as demonstrated in Table 3.6. Therefore the results presented in Fig. 3.13.a suggest that the stabilizing effect of a wavy wall extends beyond the roughness scale.

The configurations in Fig. 3.13.b demonstrate that the concave deformation results in an elevated $N$ factor growth rate over the aft panel. This is due to the highly amplified, low frequency disturbances that are unstable as a result of concave panel geometry. The one panel configuration in Fig. 3.13.b results in lower $N$ factors than the rigid wedge downstream of the panel. This is a result of the lowfrequency disturbances, that are excited by the concave panel geometry, only being unstable for short distances along the body. However, as each additional panel is added the repeated excitation of these low-frequency disturbances results in $N$ factors that attain and eventually surpass values produced by the rigid wedge. 


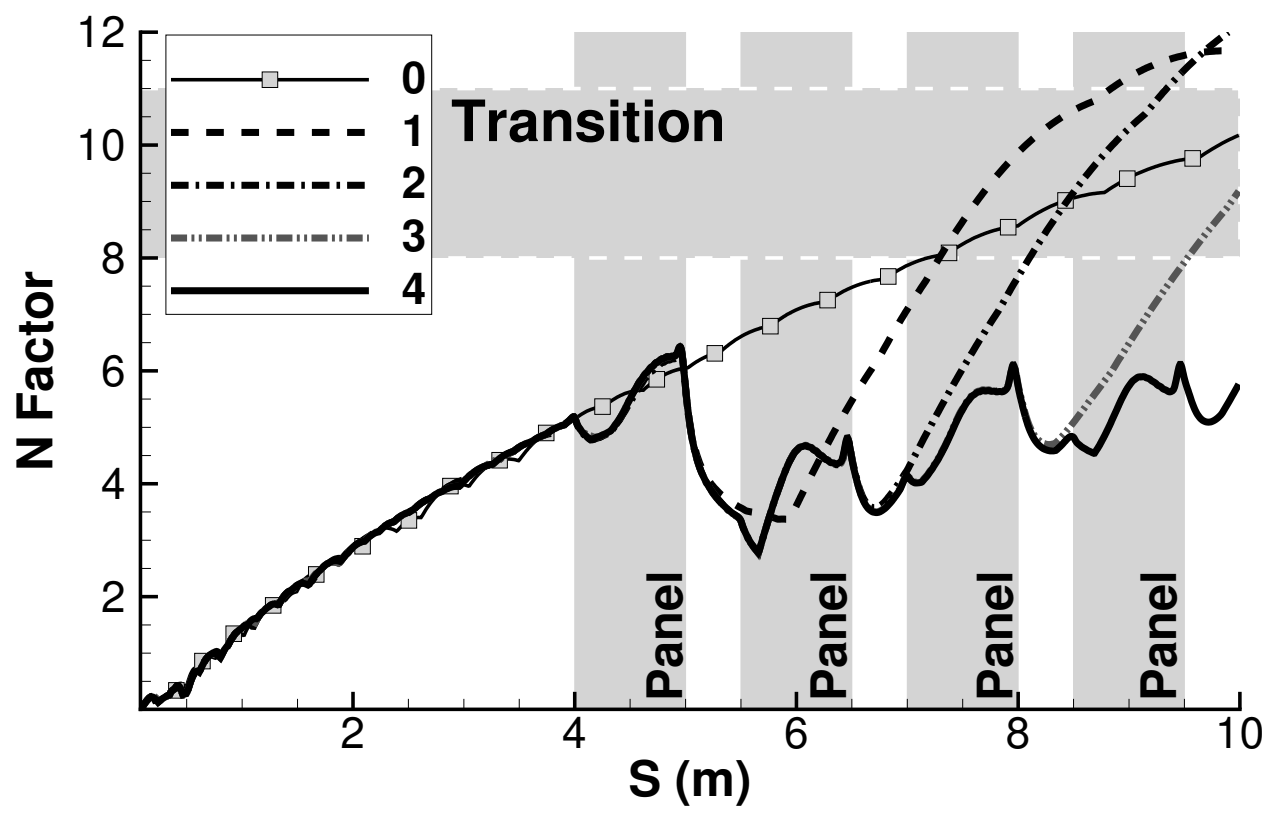

(a) Convex buckling.

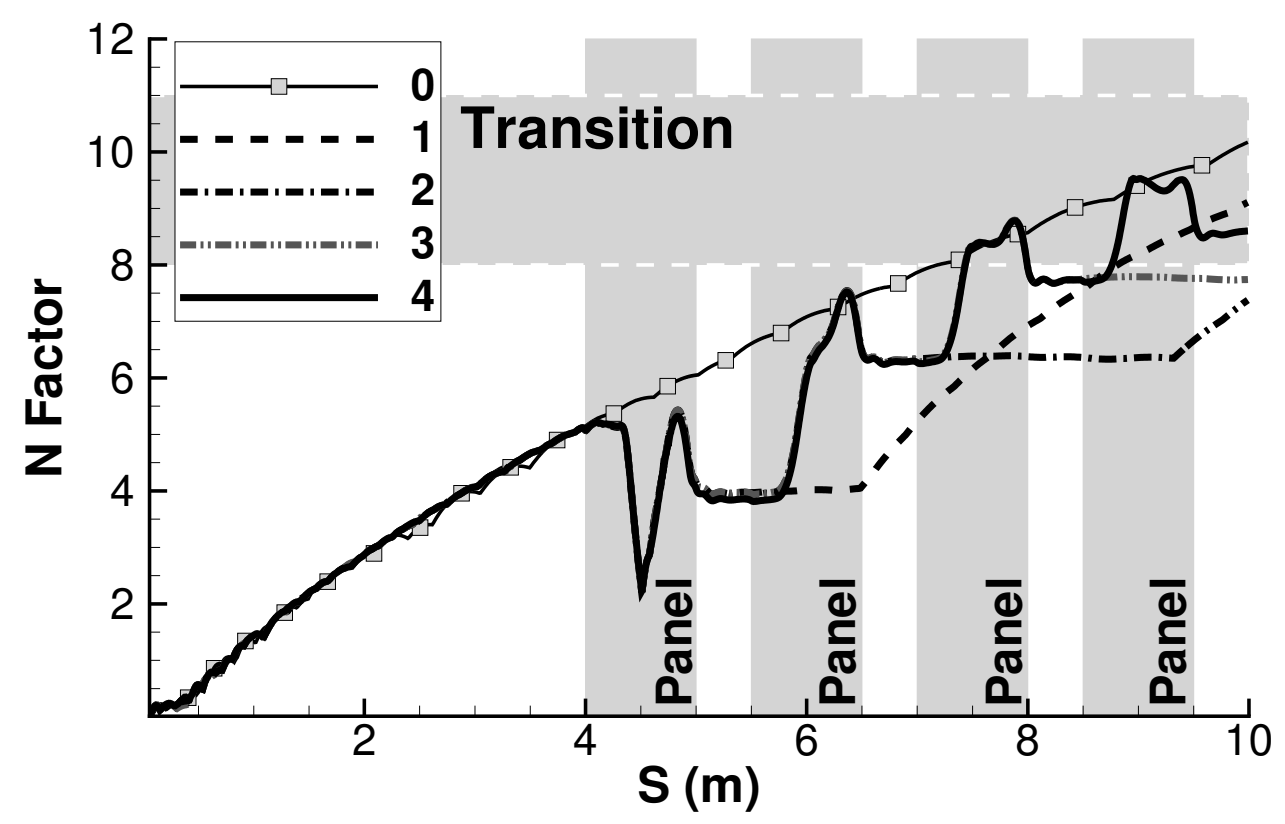

(b) Concave buckling.

Figure 3.13: $N$ factor comparison for multiple panels in series. 


\subsubsection{NASP Ramp Panel}

Boundary-layer stability throughout the constant dynamic pressure ascent can be assessed with the smooth wall, maximum $N$ factor envelopes in Fig. 3.14, where Fig. 3.14.a corresponds to the cold wall (294 K) and Fig. 3.14.b to the radiative equilibrium condition. Comparing Figs. 3.14.a and 3.14.b, it is clear that at all Mach numbers the radiative equilibrium wall temperature corresponds to lower $N$ factors. This finding is consistent with the work of Mack [42] which demonstrated that second-mode instabilities are stabilized by wall heating. Fig. 3.14 also shows that for either wall temperature the $N$ factors decrease during the ascent. Assuming the transitional $N$ factor remains constant, this decrease corresponds to improved boundary-layer stability. The results in Fig. 3.14 suggest that ascent at constant dynamic pressure has a stabilizing effect on the boundary layer. An interesting aspect is that both Mach number (increasing by a factor of 2.4) and Reynolds number (decreasing by a factor of 2.6) are changing simultaneously during the ascent. Previous studies have observed that boundary layers become more stable with increases in Mach numbers larger than four, and increases in unit Reynolds number [75]. However, as the Reynolds number is decreasing for the considered trajectory, the results in Fig. 3.14 indicate that either boundary-layer stability is more sensitive to Mach number changes, or the combined effect of changing Mach number and Reynolds number is not adequately explained through independent characterizations. 


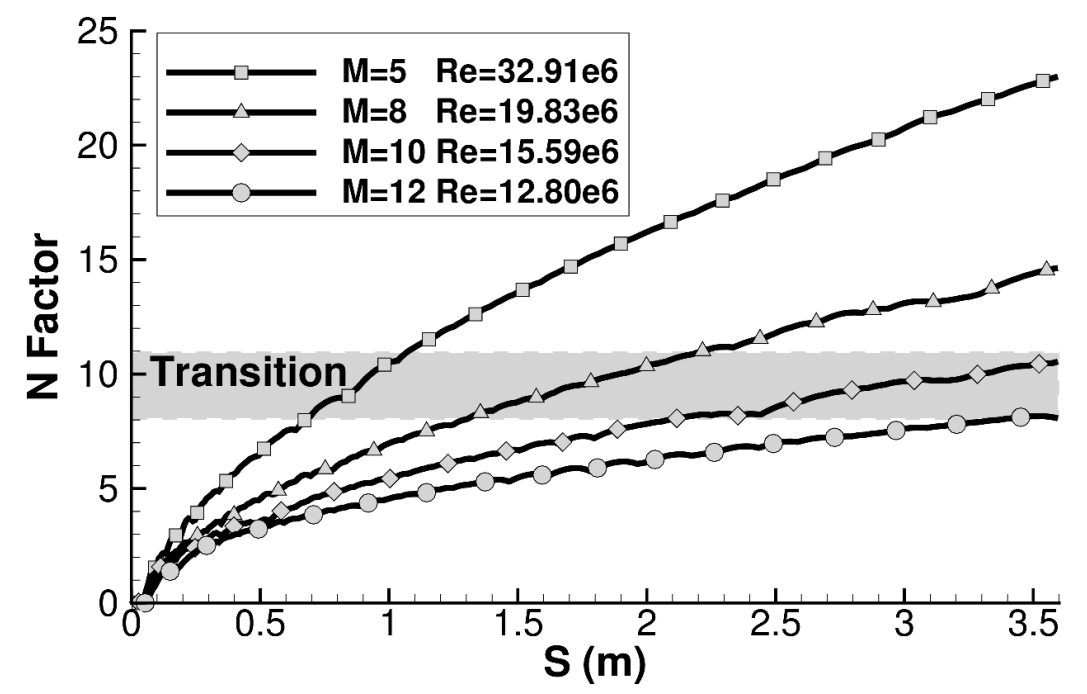

(a) Cold wall.

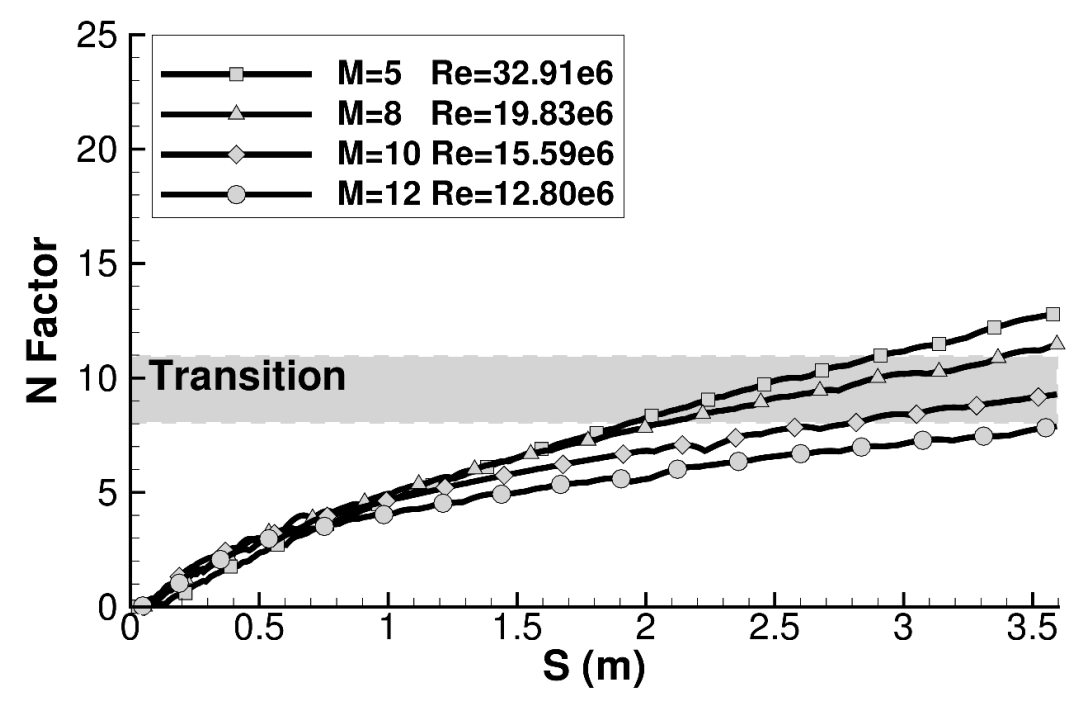

(b) Radiative equilibrium.

Figure 3.14: Maximum $N$ factors for rigid wedge during ascent. 
The impact of surface temperature on boundary-layer stability is further demonstrated in Figs. 3.15 and 3.16. In Fig. 3.15 the increased wall temperature resulting from the radiative equilibrium condition is shown to thicken the boundary layer due to the decreased fluid density. A comparison of the stability diagrams in Fig. 3.16 indicates that the higher wall temperature, corresponding to the radiative equilibrium condition, reduces the maximum amplification rate of unstable disturbances. Additionally, the thickened boundary layer reduces the frequency of the second-mode instability.

Next, consider the impact of the panel deformation on boundary-layer stability, as shown in Fig. 3.17 where cold wall $(\mathrm{CW})$ and radiative equilibrium (RE) conditions for both the rigid and deformed wedge configurations are shown in each subfigure. During the early stages of ascent at 90 seconds $(H / \delta \approx 0.09, H / L=$ $0.09 \%$ ), there is a negligible difference in $N$ factors between the rigid and deformed wedge conditions. However, by 180 seconds $(H / \delta \approx 0.60, H / L=0.99 \%)$ and beyond, the deformation clearly has a strong influence on the $N$ factor growth. The results in Figs. 3.17.b and 3.17.d indicate that at radiative equilibrium conditions the deformation produces a large reduction in $N$ factor immediately downstream of the deformation. At cold wall conditions this effect is not as prevalent and elevated $N$ factor growth is observed over the deformation. At 300 seconds, where the boundary layer is the thickest, the panel deformation tends to reduce the $N$ factors downstream for both cases. However, there is a recovery to the rigid wedge $N$ factors near the expected transition region, indicating that for the assumed panel 


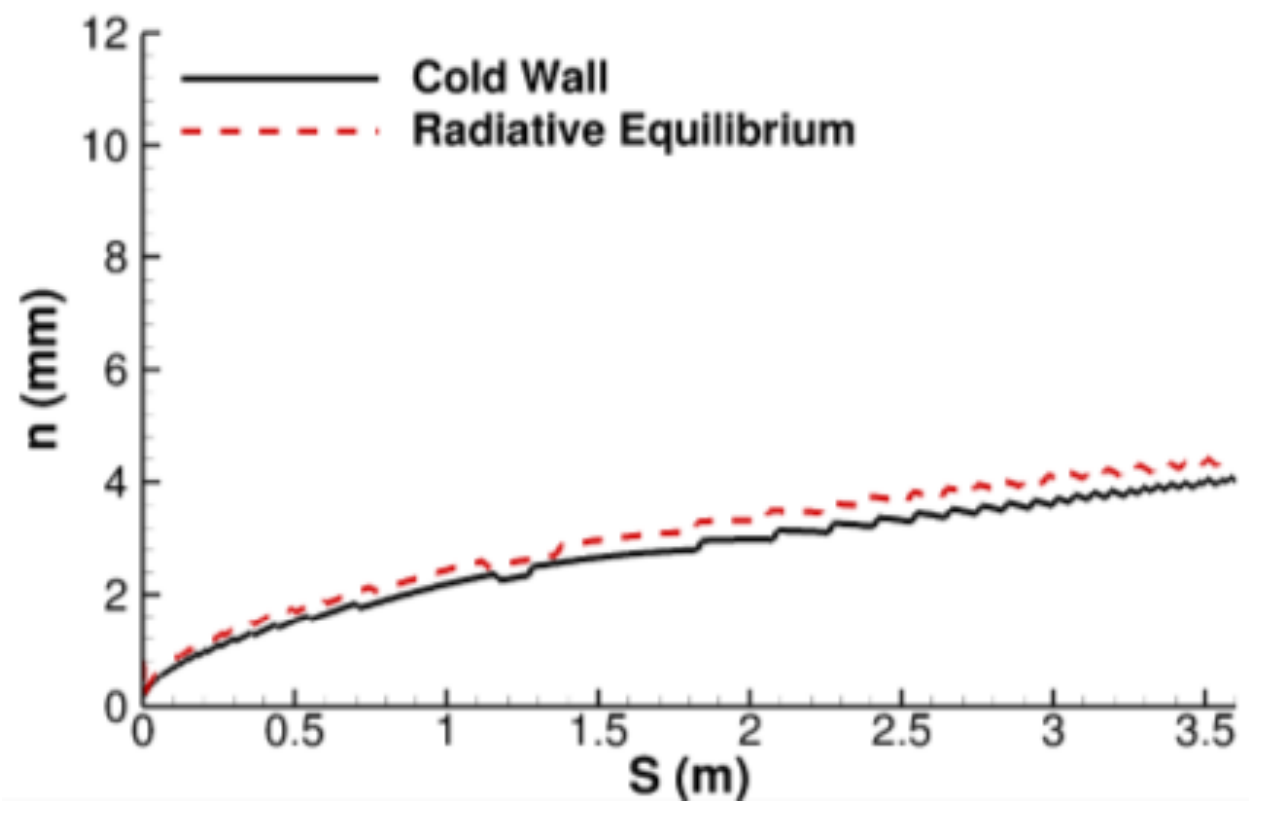

(a) $90 \mathrm{~s}\left(M=5, R e=32.91 \times 10^{6}\right)$.

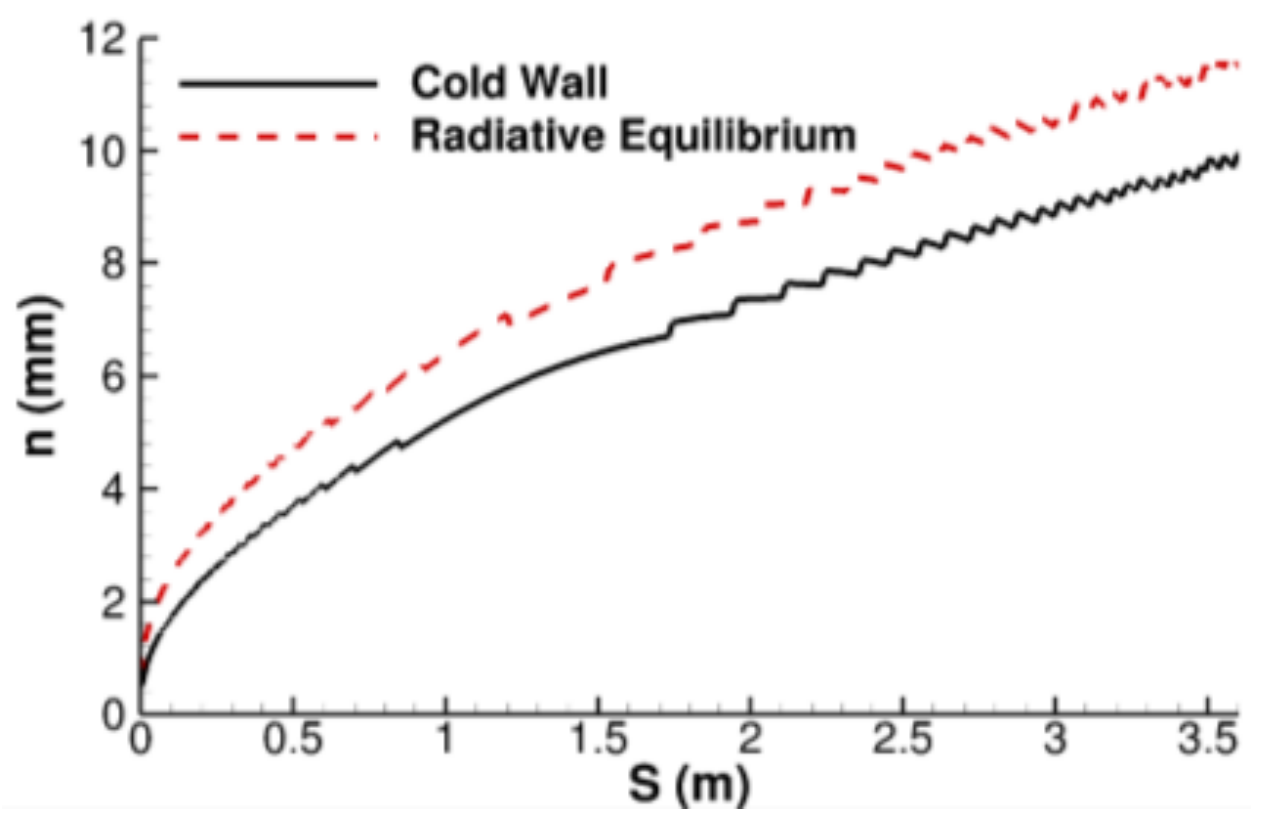

(b) $300 \mathrm{~s}\left(M=12, R e=12.80 \times 10^{6}\right)$.

Figure 3.15: Boundary-layer thickness for cold and radiative equilibrium smooth wall during ascent trajectory. 


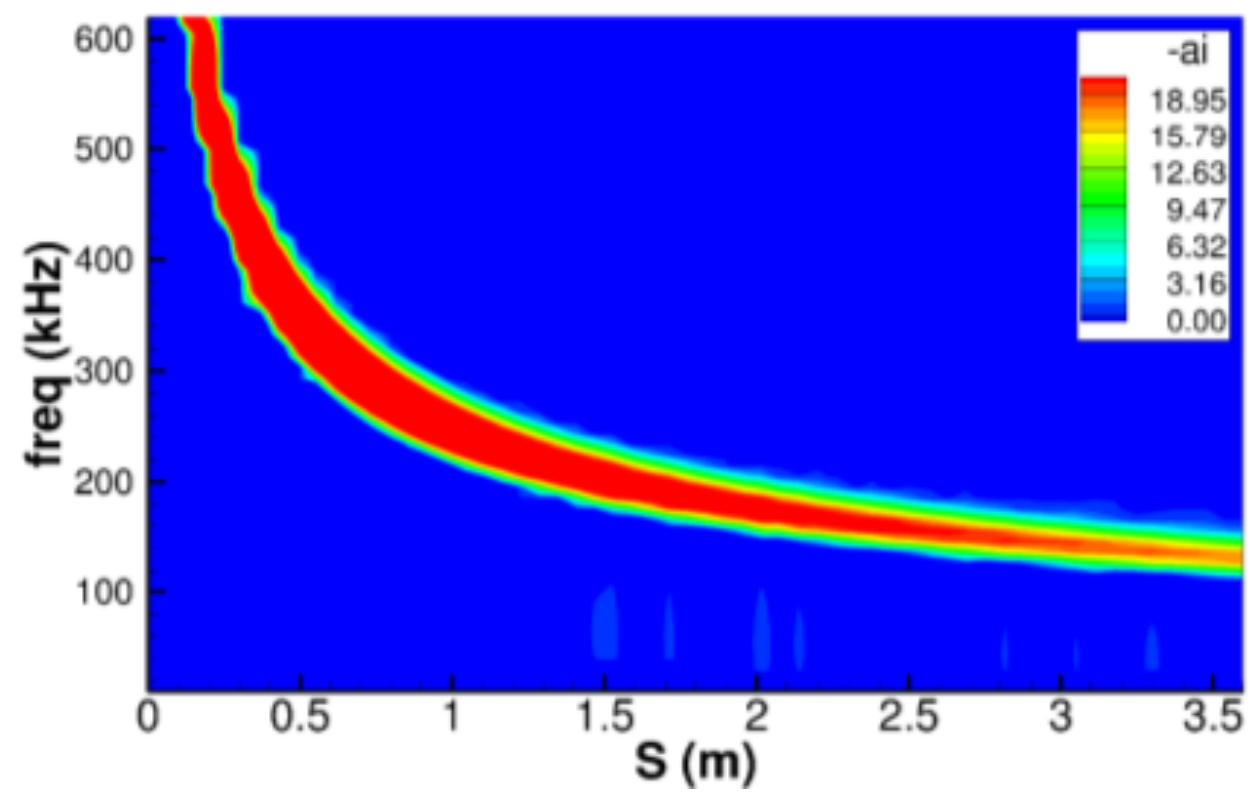

(a) Cold wall.

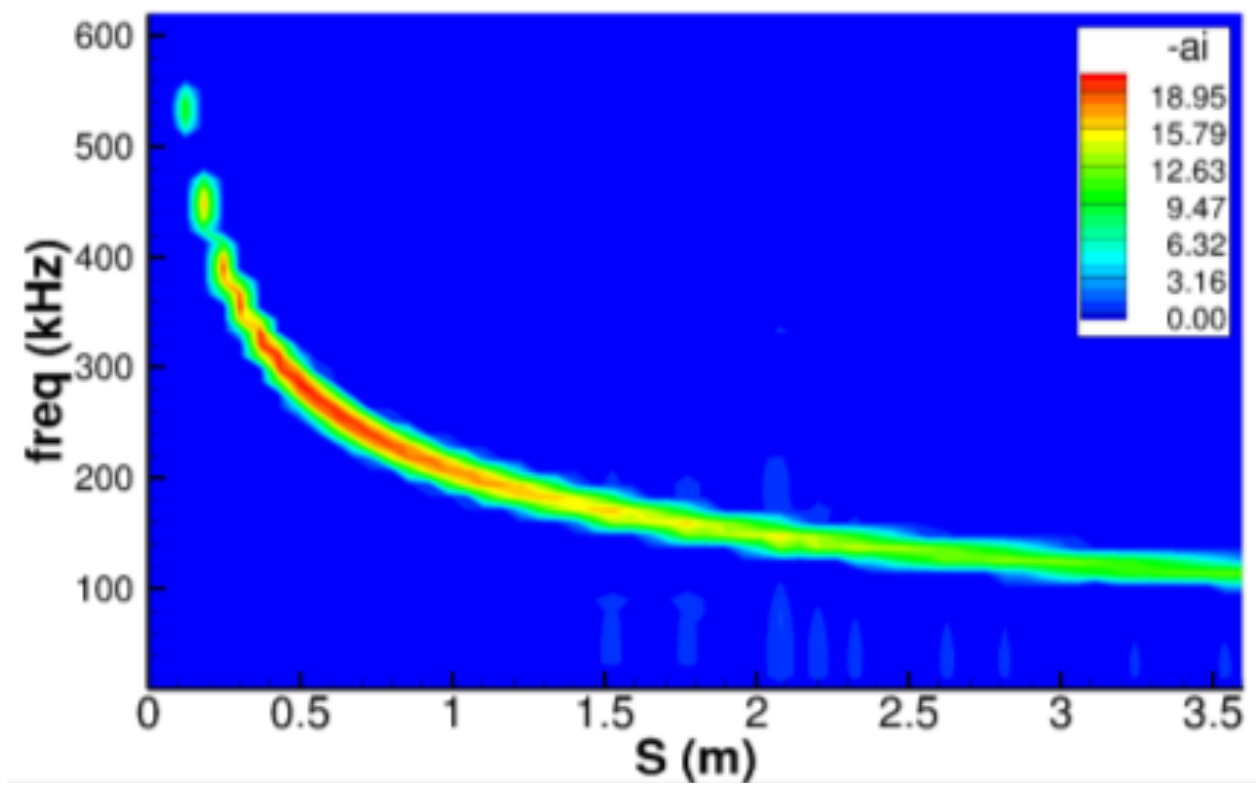

(b) Radiative equilibrium.

Figure 3.16: Stability diagrams for smooth wall geometry at $90 \mathrm{~s}$ into ascent trajectory $\left(M=5, R e=32.91 \times 10^{6}\right)$. 
location, there would be no significant impact on the boundary-layer transition point.

Another important aspect of study is the potential for higher mode deformations, which as noted occur for the NASP ramp panel beginning at 240 seconds. Thus, a comparison of Fig. 3.17.b with Fig. 3.17.c provides some insight into the importance of deformation mode on stability. Interestingly, for both wall temperature conditions, the change in deformation mode reduces the maximum $N$ factor growth rate (i.e., the slope of $N$ factor curve). Furthermore, in comparing Figs. 3.17.c and 3.17.d it is clear that the $N$ factor growth continues to decrease relative to the smooth wall case as the magnitude of the higher mode dominated deformation increases. Additional insight into this is obtained through comparison of boundary-layer thickness and LST diagrams in Fig. 3.18. Note that the shift in the deformation pattern from 180 seconds to 240 seconds has a stronger impact on the boundary-layer thickness relative to the respective rigid cases. This is despite the fact that the peak displacements are roughly equivalent between these time instants $(\sim 3 \mathrm{~mm})$, while the boundary layer is somewhat thicker at 240 seconds. The stronger impact of the deformation pattern at 240 seconds is presumably due to increased expansion over the aft section of the panel for the higher mode dominated deformation. This yields a more substantial break in disturbance frequencies exhibiting unstable growth, as highlighted by the LST results. 


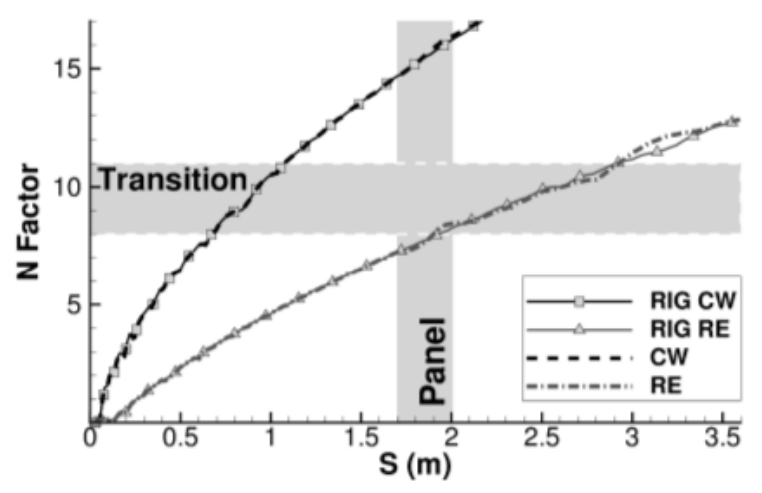

(a) 90 seconds.

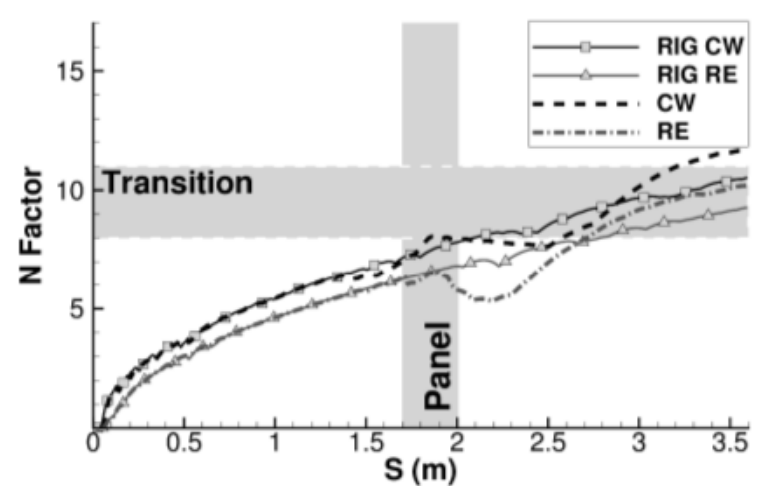

(c) 240 seconds.

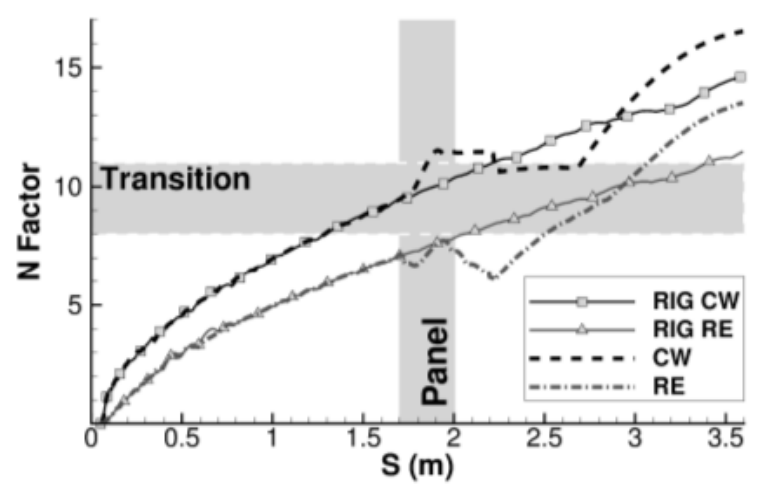

(b) 180 seconds.

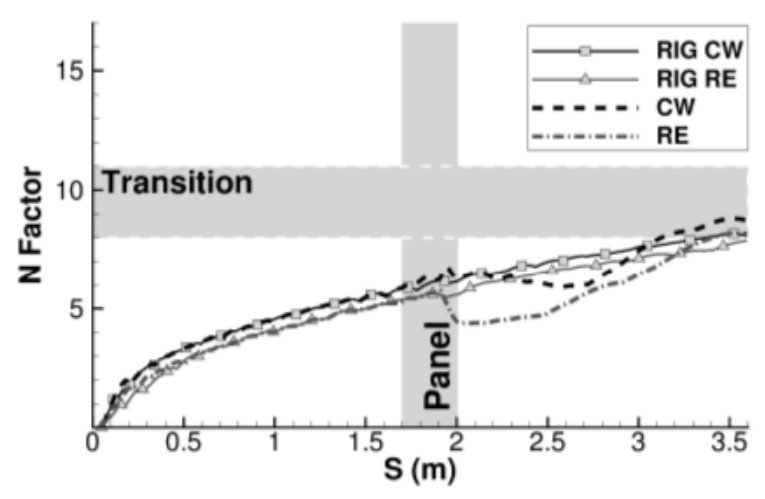

(d) 300 seconds.

Figure 3.17: Maximum $N$ factors for panel response during ascent. 


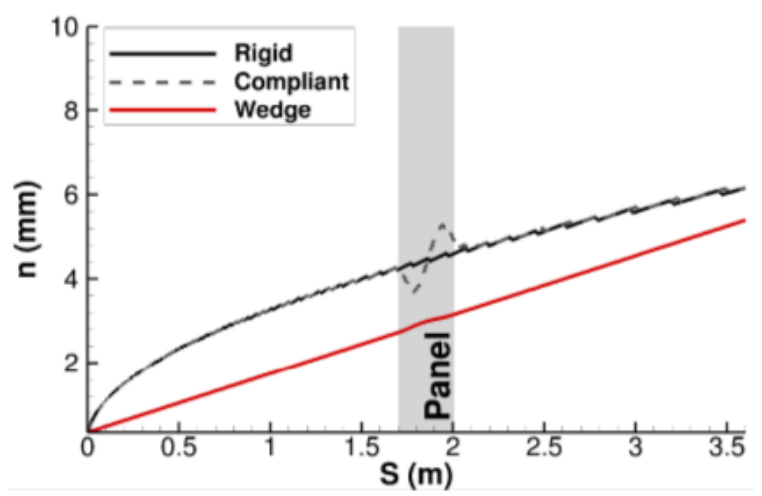

(a) Boundary-layer thickness at $180 \mathrm{~s}$.

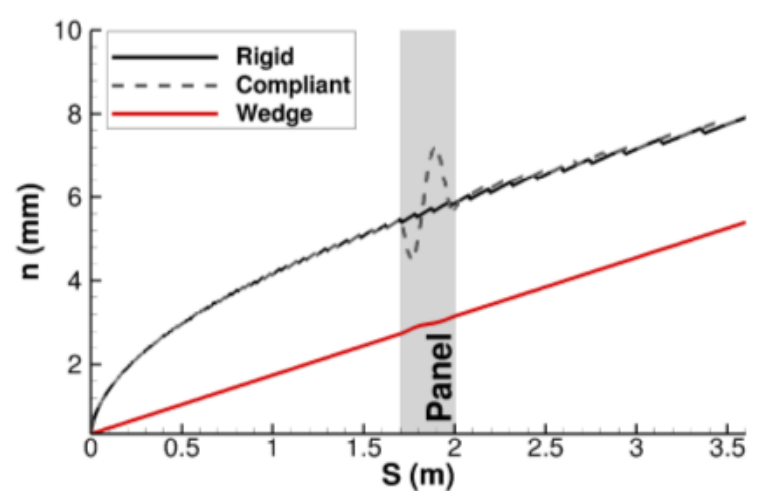

(c) Boundary-layer thickness at $240 \mathrm{~s}$.

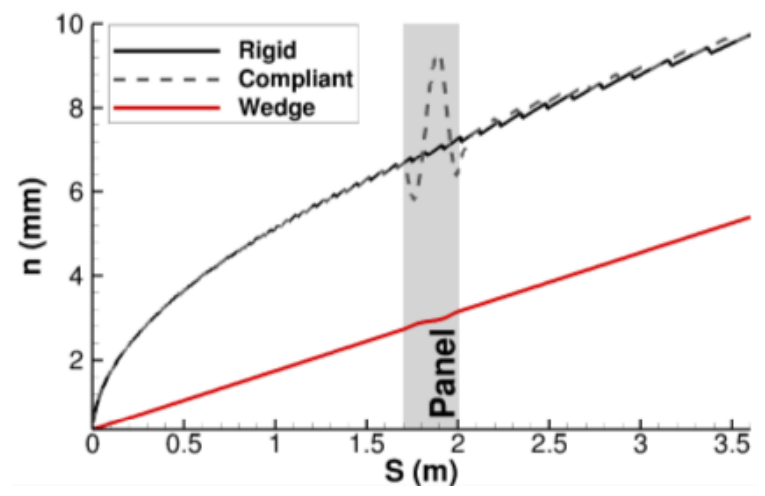

(e) Boundary-layer thickness at $300 \mathrm{~s}$.

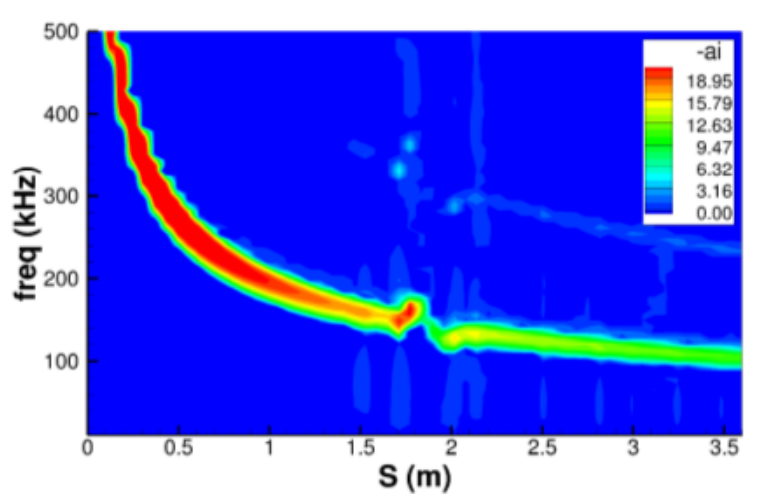

(b) LST diagram at $180 \mathrm{~s}$.

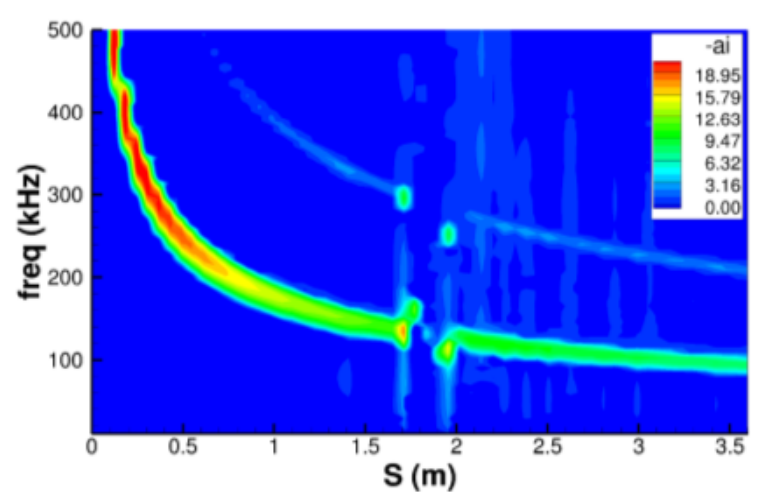

(d) LST diagram at $240 \mathrm{~s}$.

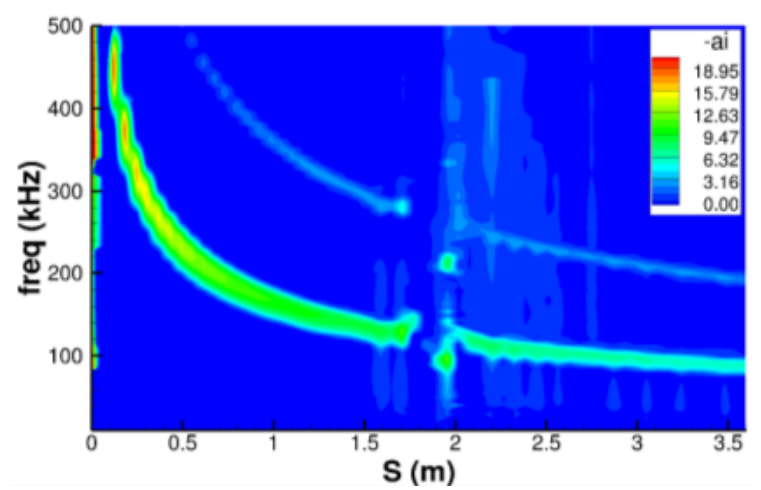

(f) LST diagram at $300 \mathrm{~s}$.

Figure 3.18: Impact of panel deformation pattern on boundary-layer growth and stability for cold wall. 


\subsubsection{Spherical Domes}

LPSE results for the six dome geometries described in Table 3.4 are shown in Fig. 3.19, which provides a comparison in the maximum $N$ factor growth for the rigid and deformed wedge at both cold wall (CW) and radiative equilibrium (RE) wall temperature conditions. Recall for the deformed wedge configurations, both "simply-supported" (SS) and "clamped" (CC) deformations are considered.

As previously discussed in Section 3.3.2, the results in Fig. 3.19.a-d further demonstrate that inclusion of the deformation increases $N$ factors in the vicinity of the panel at the cold wall condition and decreases $N$ factors for the radiative equilibrium case. This illustrates the coupled effect of surface deformation and wall temperature as the influence of the deformation on stability varies depending on the specified wall temperature. Finally, results in Fig. 3.19 suggest the structural boundary condition has a negligible effect on the boundary-layer stability as both the simply-supported and clamped configurations result in similar $N$ factor growth envelopes. A similar result was observed by Marxen and Iaccarino [157] for a Mach 4.8 flow comparing a roughness element with smooth boundary conditions and that of a rectangular roughness element with the same height and cross-sectional area.

In comparing the subfigures in Fig. 3.19, the rows correspond to magnitude of the deformation and columns correspond to length and leading edge location. Thus, shifting top to bottom corresponds to increasing panel bowing, while left to 
right corresponds to increasing both the deformation length and shifting the leading edge forward. As expected, increasing the size of the deformation (moving left to right) increases its impact on the $N$ factor growth. The same is true for increasing deformation height (moving top to bottom). For both temperature conditions, it can be seen that increasing the deformation height results in decreased $N$ factor growth over and downstream of the deformation. In particular, there is a dramatic shift in $N$ factor growth when the displacement ratio $(H / L)$ increases from $1.43 \%$ to $2.86 \%$. Furthermore, the reduction in $N$ factor growth is most dramatic for dome 6, which corresponds with the largest deformation in Fig. 3.19. The decrease in $N$ factor with increasing displacement agrees with the work of Fong et al. [158] who found that for single roughness elements $(L / \delta=4)$ in Mach 5.92 flow, as $H / \delta$ increased from 0.25 to 0.625 , flow disturbances were more effectively damped. 


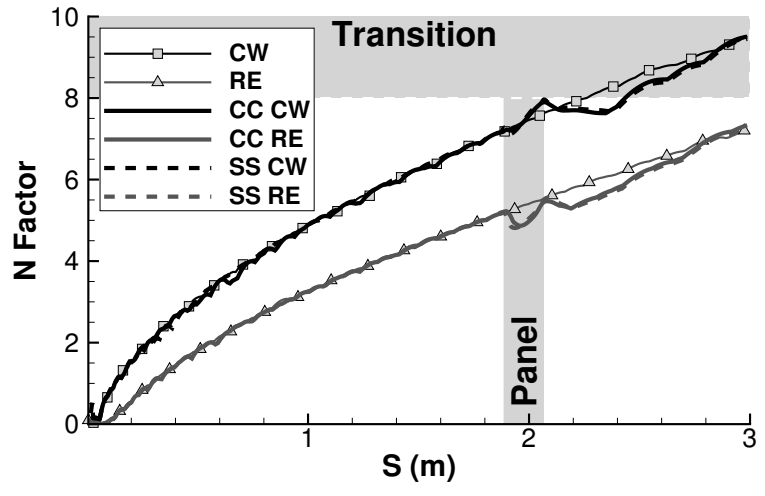

(a) Dome $1(\mathrm{H} / \mathrm{L}=0.007)$.

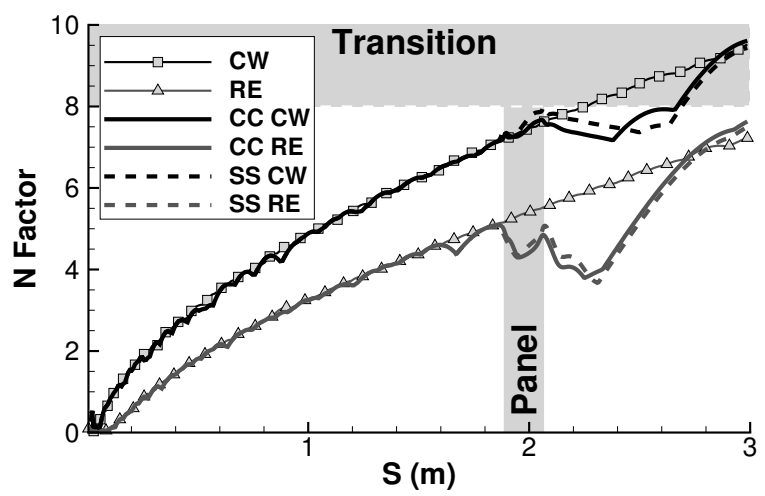

(c) Dome $2(\mathrm{H} / \mathrm{L}=0.0143)$.

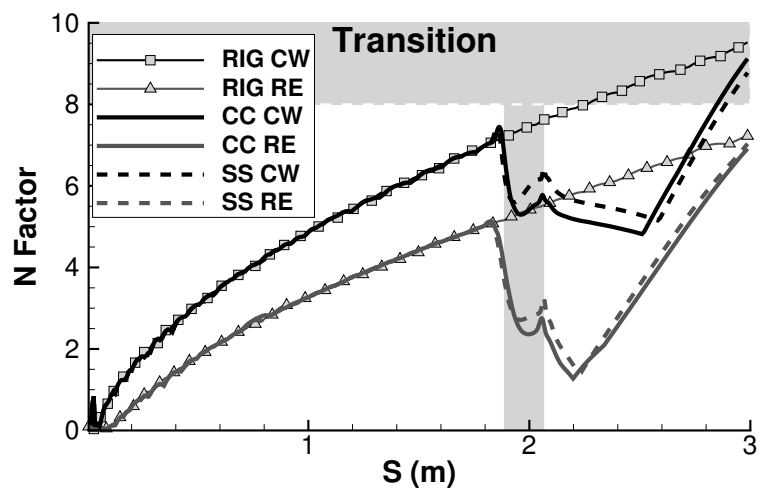

(e) Dome $3(\mathrm{H} / \mathrm{L}=0.0286)$.

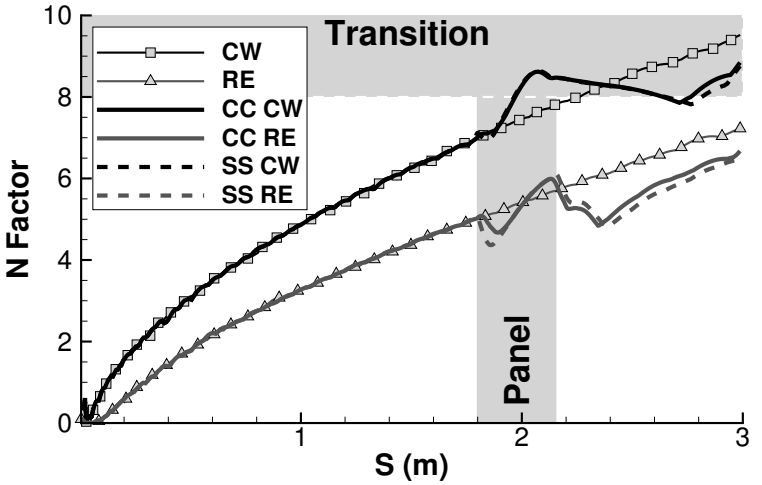

(b) Dome $4(\mathrm{H} / \mathrm{L}=0.007)$.

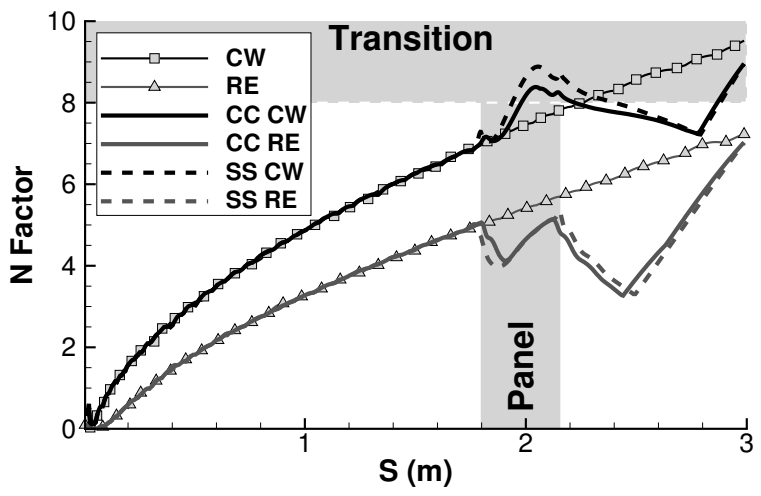

(d) Dome $5(\mathrm{H} / \mathrm{L}=0.0143)$.

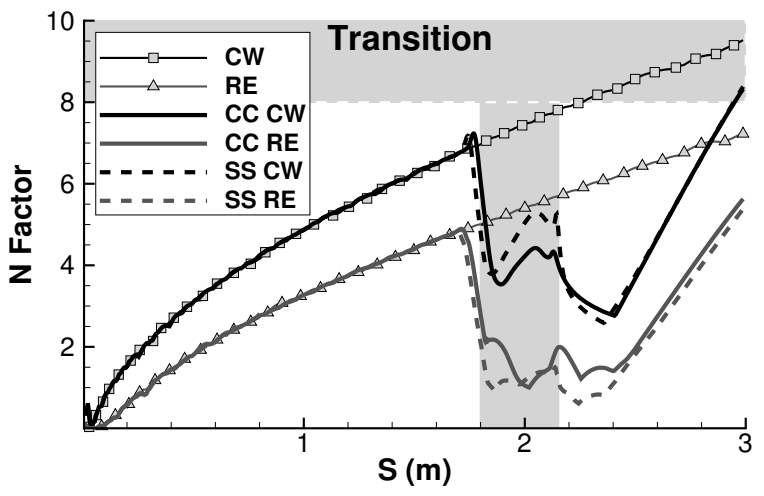

(f) Dome $6(\mathrm{H} / \mathrm{L}=0.0286)$.

Figure 3.19: Maximum $N$ factor growth for domes 1 to 6 . 


\section{Chapter 4}

\section{Impact of Boundary-Layer Transition on \\ Structural Response}

The dynamic response of a representative hypersonic vehicle panel, subject to combined aerodynamic and thermal loading, is computed for laminar, transitional, and turbulent flow. As shown in Fig. 2.15, the panel is located on a 2-D surface inclined to the freestream, representing the forebody of a supersonic/hypersonic vehicle. The freestream conditions and geometry of the simply-supported panel are provided in Section 4.1. Panel responses are obtained using the aerothermoelastic framework in Fig. 2.6, where the transition onset and length are prescribed prior to the simulation. The effect of varying transition onset location, transition length, and the overshoot magnitude in heat flux and fluctuating pressure on the structural response is examined. Additionally, results are presented for cases that neglect and include the effects of transitional overshoot. 


\subsection{Problem Description}

The freestream conditions and simply-supported panel geometry are listed in Tables 4.1 and 4.2. The mass and stiffness-proportional Rayleigh damping is specified such that $2 \%$ of the critical damping is achieved at both the fundamental natural frequency and at $1000 \mathrm{~Hz}$ to provide viscous damping of the higher frequencies. The temperature-dependent material properties of the aluminum panel are listed in Table 4.3. The density and Poisson's ratio are assumed to be constant over the operational range of temperature. The pinned aluminum panel examined here has a maximum modal frequency of $3.80 \mathrm{kHz}$. While this configuration does not represent an actual structure intended for use on a hypersonic vehicle, it has been thoroughly examined in past works $[17,35]$ and has been shown to provide a demonstration of the coupled (fluid-thermal-structural) response over a relatively short time record [35].

Table 4.1: Freestream conditions.

\begin{tabular}{lc}
\hline \hline Parameter & Value \\
\hline Mach number (freestream) & 4.00 \\
Mach number (post oblique shock) & 3.64 \\
Altitude, km & 30.0 \\
Unit Reynolds number, $\times 10^{6} \mathrm{~m}^{-1}$ & 1.46 \\
\hline \hline
\end{tabular}


Table 4.2: Panel geometry.

\begin{tabular}{lc}
\hline \hline Parameter & Value \\
\hline Leading edge location, $\mathrm{m}$ & 1.00 \\
Length, $\mathrm{m}$ & 1.00 \\
Thickness, mm & 2.50 \\
Density, $\mathrm{kg} / \mathrm{m}^{3}$ & 2768 \\
Poisson's ratio & 0.325 \\
\hline \hline
\end{tabular}

Table 4.3: Temperature dependence of material properties of Aluminum (Al-7075).

\begin{tabular}{lllll}
\hline \hline$T$ & $c$ & $k$ & $E$ & $\alpha$ \\
$\mathrm{K}$ & $\mathrm{J} / \mathrm{kg} \mathrm{K}$ & $\mathrm{W} / \mathrm{m} \mathrm{K}$ & $\mathrm{GPa}$ & $\mu \mathrm{m} / \mathrm{mK}$ \\
\hline 300 & 850.99 & 132.05 & 72.86 & 22.36 \\
350 & 892.44 & 138.19 & 70.84 & 22.73 \\
400 & 933.89 & 143.17 & 67.53 & 23.21 \\
450 & 975.33 & 146.99 & 62.49 & 23.74 \\
500 & 1016.78 & 149.67 & 55.40 & 24.25 \\
\hline \hline
\end{tabular}

\subsection{Panel Response for Laminar and Turbulent Flow}

Panel responses corresponding to laminar and turbulent flow are provided as a means to quantify the effect of transitional fluid loading on the panel. Time histories of the average temperature and mid-panel displacement, corresponding to each flow condition, are shown in Fig. 4.1. Note that the flutter onset times are provided in Fig. 4.1.a. A comparison of the average temperatures in Fig. 4.1.a indicates that the elevated aerodynamic heating, which is a product of the turbulent boundary layer, increases the average temperature throughout the panel at a much faster rate. This produces a larger thermal force that increases the rate of deformation (Fig. 4.1.b). The turbulent loading reduces the onset time to flutter (Fig. 4.1.a) 
to approximately $17 \%$ of the laminar value. While the time to flutter is significantly different for the panel subject to laminar and turbulent loading, the characteristic response at flutter onset is similar. The horizontal lines in Fig. 4.1 denote the average temperature and center displacement at flutter onset for the panel subject to turbulent flow. As shown in Fig. 4.1, the laminar and turbulent panel responses are within $3.5 \mathrm{~K}$ and $0.83 \mathrm{~mm}(w / h=0.33)$ of one another at flutter onset. This indicates that the difference in the laminar and turbulent aerothermodynamic loads primarily impacts the rate of the structural response.

\subsection{Transitional Overshoot Characterization}

As described in Section 2.3.3, overshoot in a transitional load (heat flux or RMS pressure) is produced through an intermittency-based blending of laminar and turbulent flow solutions, where the turbulent boundary layer originates at the transition onset location. As this blending is a function of the intermittency, the overshoot magnitude is dependent on the length of the transition region.

The average percent overshoot is provided in Fig. 4.2 in terms of heat flux (Fig. 4.2.a) and RMS pressure (Fig. 4.2.b) as a function of the transition length $\Delta x_{t}$. The overshoot is defined as shown in Eq. (4.1):

$$
f_{\text {overshoot }}=\frac{f_{\text {max }}(x)-f_{\text {turb }}}{f_{\text {turb }}}
$$




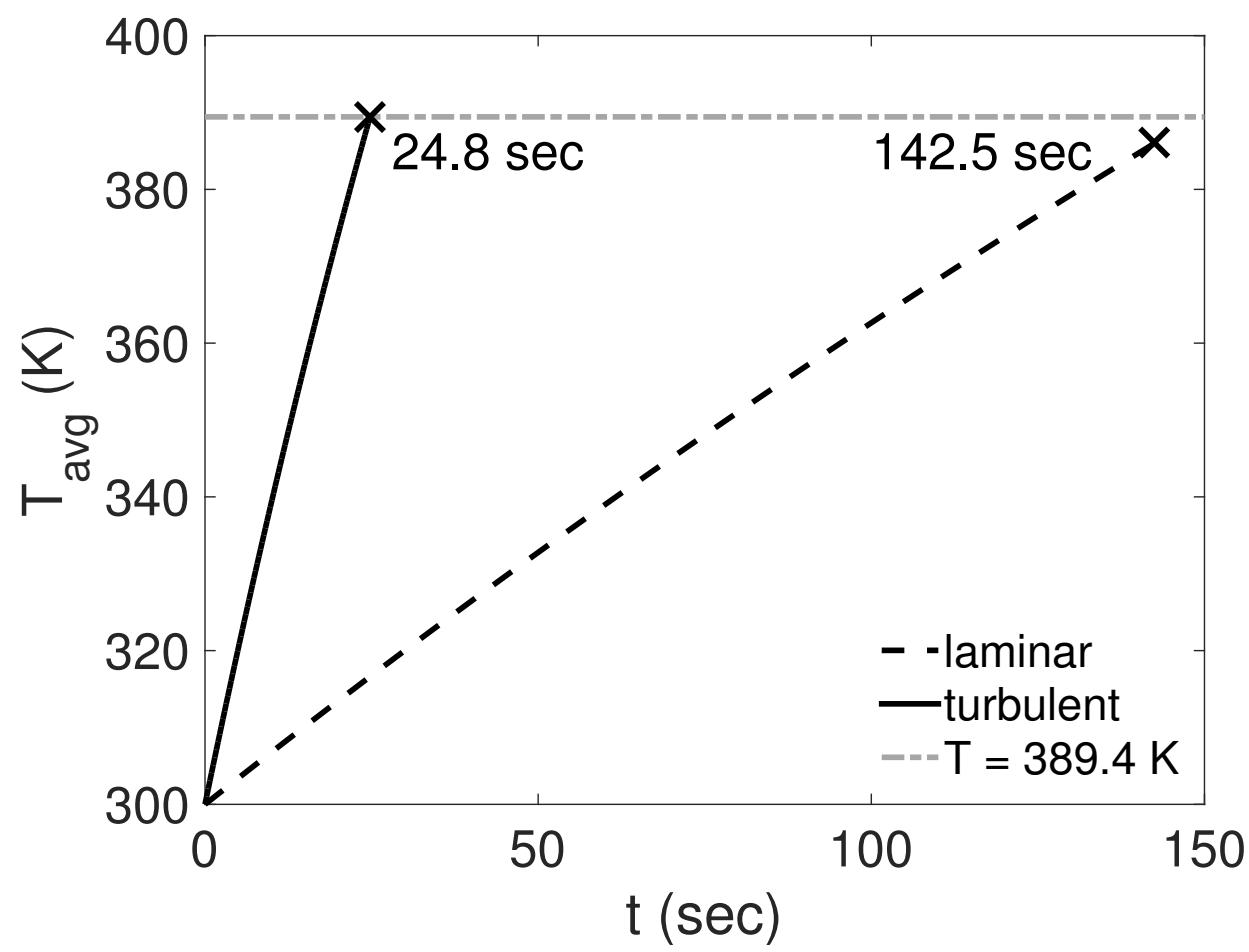

(a) Average temperature.

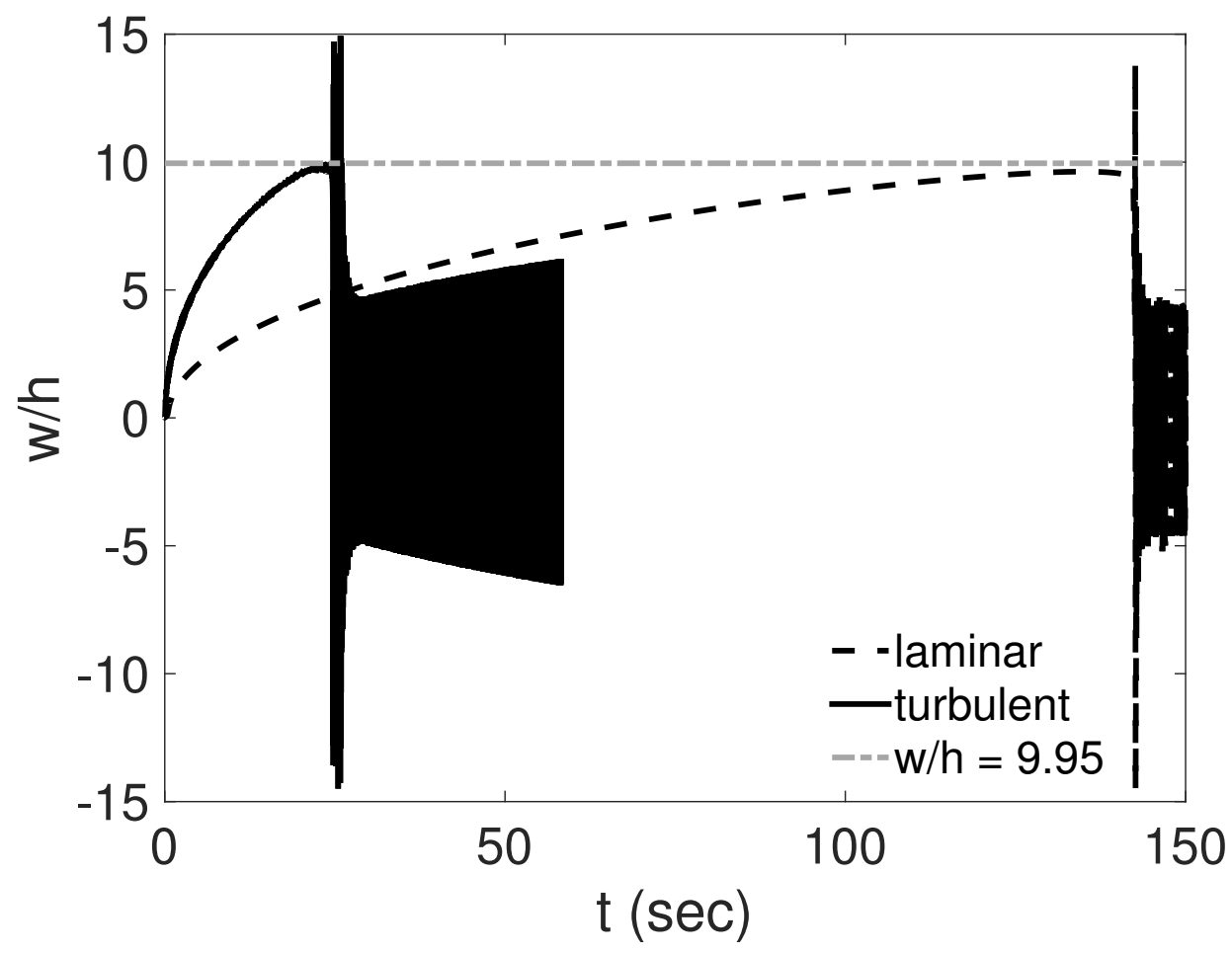

(b) Center displacement.

Figure 4.1: Panel response for laminar and turbulent flows. Times in Fig. 4.1.a correspond to laminar and turbulent flutter onsets. 
where $f(x)$ corresponds to either the heat flux or RMS pressure distribution, $f_{\max }$ is the maximum value over the length of the panel, and $f_{\text {turb }}$ is the turbulent value at trailing edge. Note that the percentages in Fig. 4.2 correspond to the undeformed panel. Also, the variation in heat flux overshoot is negligible for cases with the same $\Delta x_{t}$ but different $x_{t}$. However, for pressure there is a variation of roughly $10 \%$ from the average values shown.

The heat flux and RMS pressure overshoot decreases with increasing transition length. Previous experimental studies, on hypersonic flow over flat plates [159] and cones $[25,121]$, have observed heat flux overshoot ranging from 50 to $70 \%$. Therefore, the shorter transition lengths considered in this study $\left(\Delta \bar{x}_{t} \leq 0.10\right)$ may over predict the peak heat flux. As transitional RMS pressures have been recorded as large as three times the turbulent conditions [160], the overshoot percentages in Fig. 4.2.b all fall within the experimentally observed range.

\subsection{Effect of Transition Length on Panel Response}

The impact of transition length on the stable response of the panel is shown in Fig. 4.3 in terms of maximum deformation, slope, temperature and thermal gradient. Results are presented for transitional cases with onset at $\bar{x}_{t}=0.1$ and the end of transition at $\bar{x}_{t e}=0.25$ and 0.90 . These configurations correspond to the minimum and maximum transition lengths, considered in this study, with experimentally bounded heat flux overshoot. For each transition length, results are presented for 


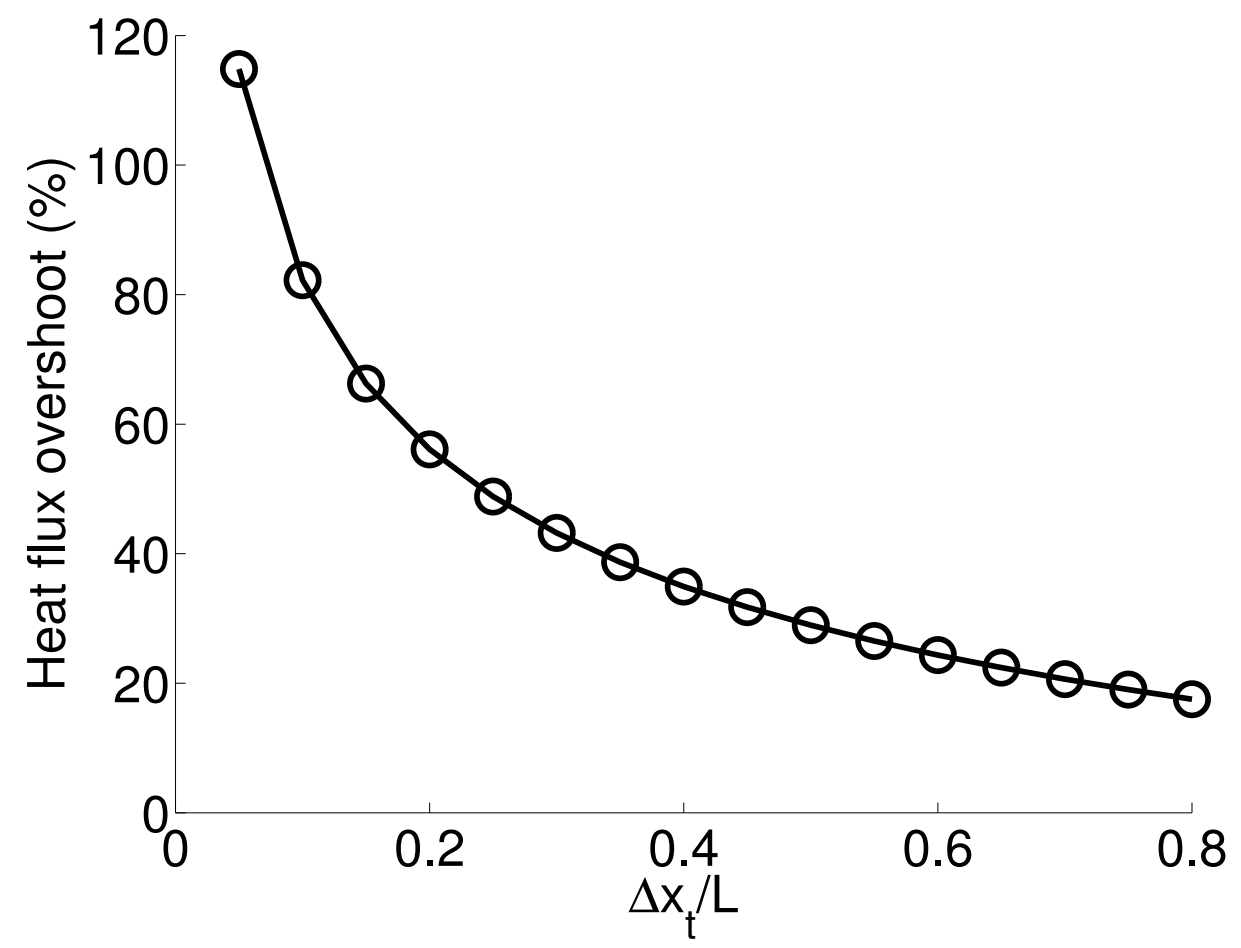

(a) Heat flux.

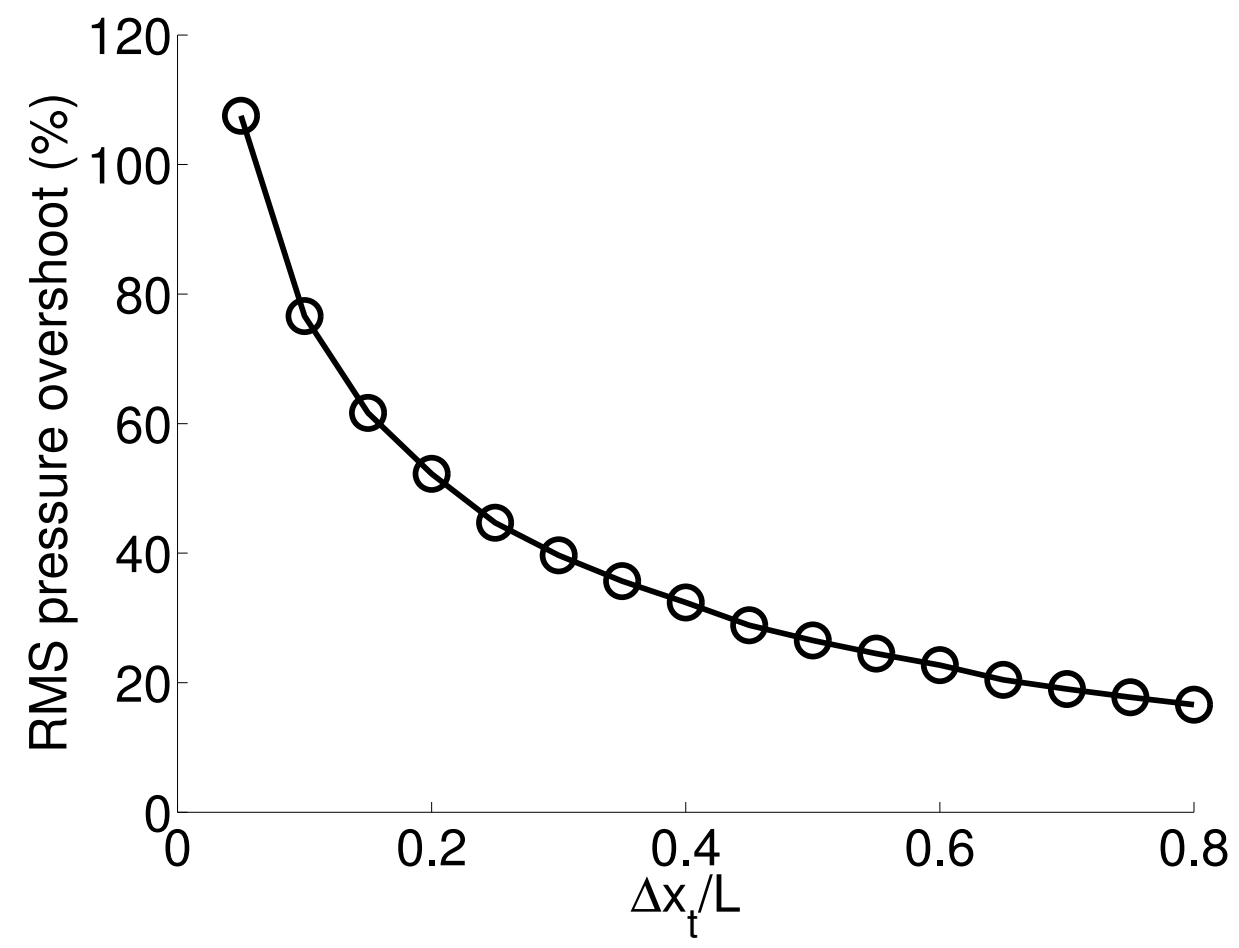

(b) RMS pressure.

Figure 4.2: Average overshoot percentage for transitional cases with the same $\Delta x_{t}$ but different $x_{t}$. 
no overshoot and overshoot in heat flux and fluctuating pressure. Panel responses are shown for the first $23 \mathrm{~s}$, as the transitional overshoot case with $\bar{x}_{t}=0.1, \bar{x}_{t e}=$ 0.25 undergoes flutter at 23.3 seconds.

A comparison of the peak displacements for the transitional responses in Fig. 4.3.a indicates that as the end of transition moves towards the front of the panel, the maximum deformation increases. Further, for fixed transition onset and end locations, accounting for overshoot produces larger displacements than the case of no overshoot. Similar trends are observed for the panel slope in Fig. 4.3.b. As the rate of deformation is driven in part by the thermal load, the increased displacement is a product of the elevated aerodynamic heating due to the larger region of turbulent loading and the increased heat flux overshoot resulting from the shorter transition length. In Figs. 4.3.a and 4.3.b, the transitional cases are bounded by the laminar and turbulent responses. Thus, these results suggest that the assumption of fully turbulent flow provides a conservative estimate of the peak displacement and slope of the panel.

The thermal response of the panel, in Figs. 4.3.c and 4.3.d, indicates that transitional fluid loading can produce maximum temperatures and thermal gradients that are not bounded by the turbulent response. Specifically, the transitional overshoot configuration with $\bar{x}_{t}=0.1$ and $\bar{x}_{t e}=0.25$, in Fig. 4.3.c, has peak temperatures exceeding that produced by turbulent flow, by up to $9 \%$, throughout the first $23 \mathrm{~s}$ of response. The results in Fig. 4.3.d illustrate that all the transitional cases produce thermal gradients exceeding both laminar and turbulent flows, with the 
shortest transition length providing the largest temperature gradients. The thermal gradient for the laminar response is not visible in Fig. 4.3.d as the peak magnitude is less than $1 \mathrm{~K} / \mathrm{m}$. The elevated temperatures and thermal gradients produced by the transitional heat flux result in local regions along the panel with large variation in temperature-dependent material properties, as well as locations where material temperature limits may be exceeded.

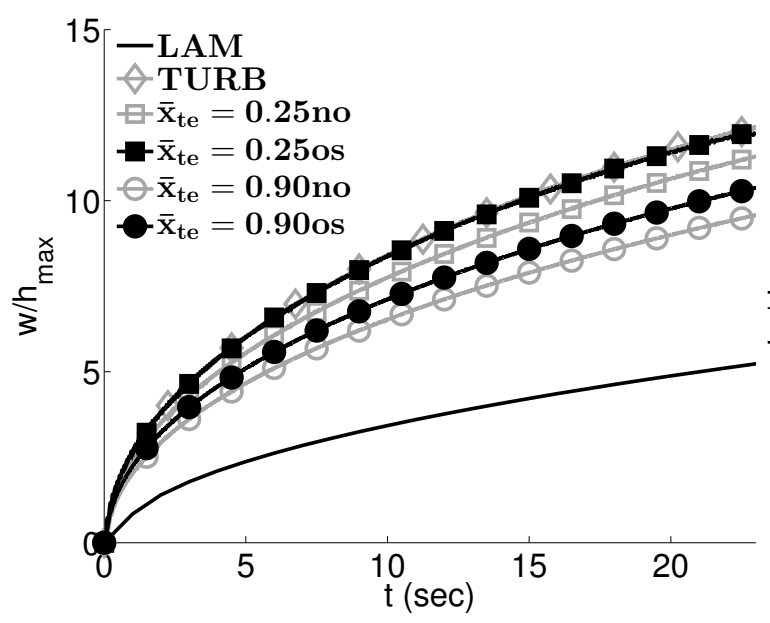

(a) Maximum displacement.

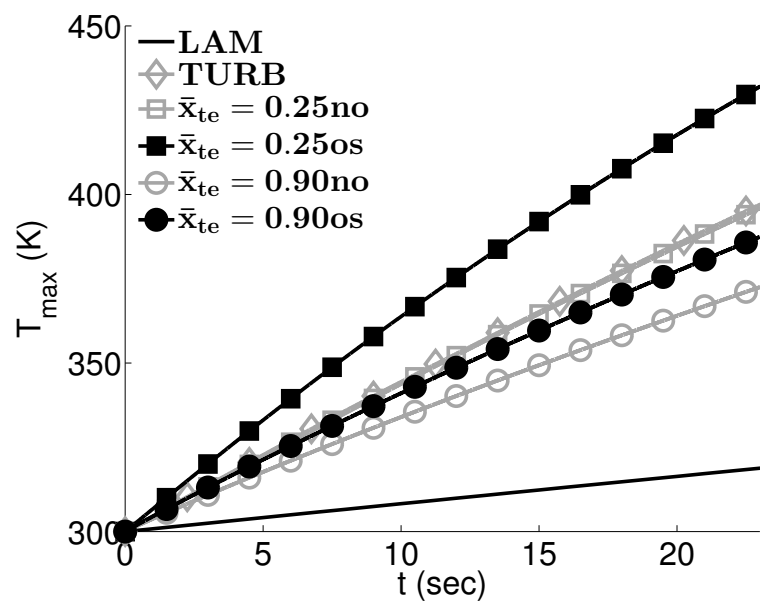

(c) Maximum temperature.

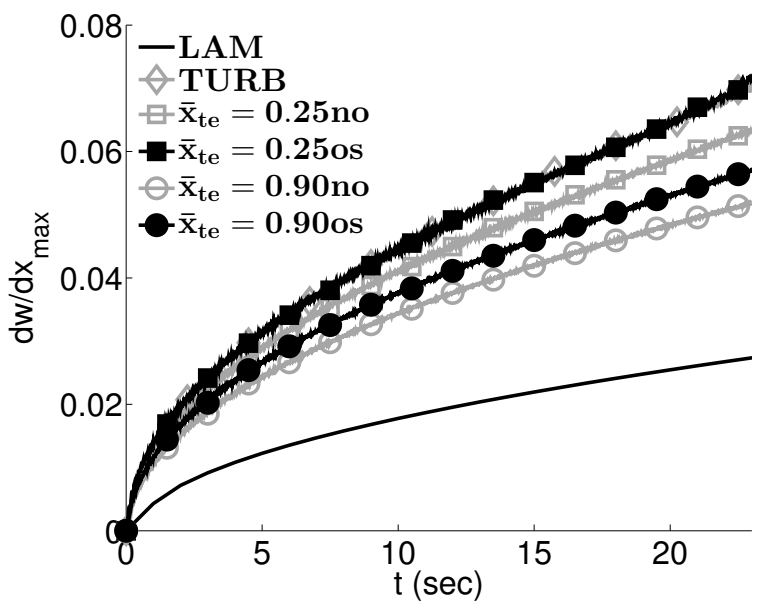

(b) Maximum slope.

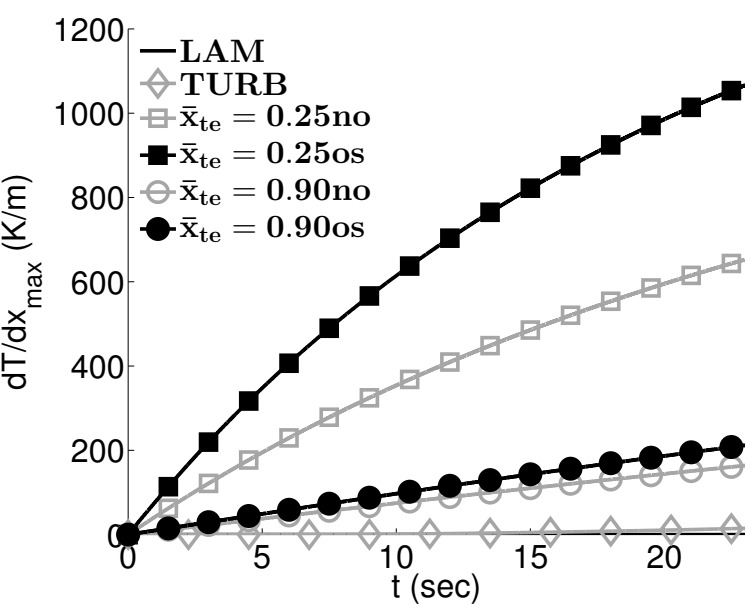

(d) Maximum chordwise temperature gradient.

Figure 4.3: Variation in panel response due to transition length $\left(\bar{x}_{t}=0.1\right)$ : no overshoot "no" (gray lines and open markers), and overshoot "os" (black lines and closed markers). 
Snapshots of the fully turbulent and transitional panel responses at $23.0 \mathrm{~s}$ are provided in Fig. 4.4. The transitional loading $\left(\bar{x}_{t}=0.1, \bar{x}_{t e}=0.25\right)$ corresponds to the minimum transition length, overshoot configuration in Fig. 4.3. A comparison of the responses in Fig. 4.4 further indicates that the turbulent assumption conservatively predicts the panel displacement despite the region of elevated heating and increased spatial variation in the through-thickness average temperature resulting from the transitional loading. This is a result of the strong dependence of the deformation on the chordwise average temperature of the panel that, as shown in the legend of Fig. 4.4.b, is nearly identical for the turbulent and transitional responses. As discussed in Section 2.3.5, the dependence of the deformation on the chordwise average temperature is due to the assumption of immovable supports in the structural equation of motion [35]. Therefore, accounting for more representative boundary conditions (clamped in rotation, axial expansion, etc.) could potentially alter the impact of the transitional loading on the response of the structure.

\subsection{Effect of Transition Location on Panel Response}

The effect of transition location on the response of the panel is shown in Fig. 4.5. Transitional cases correspond to onset at $\bar{x}_{t}=0.1$ and 0.7 , with transition length of $\Delta \bar{x}_{t}=0.15$. For each transition location, results are provided corresponding to no overshoot and overshoot in heat flux and fluctuating pressure.

Similar to the results presented in Section 4.4, the peak displacement and slope, 


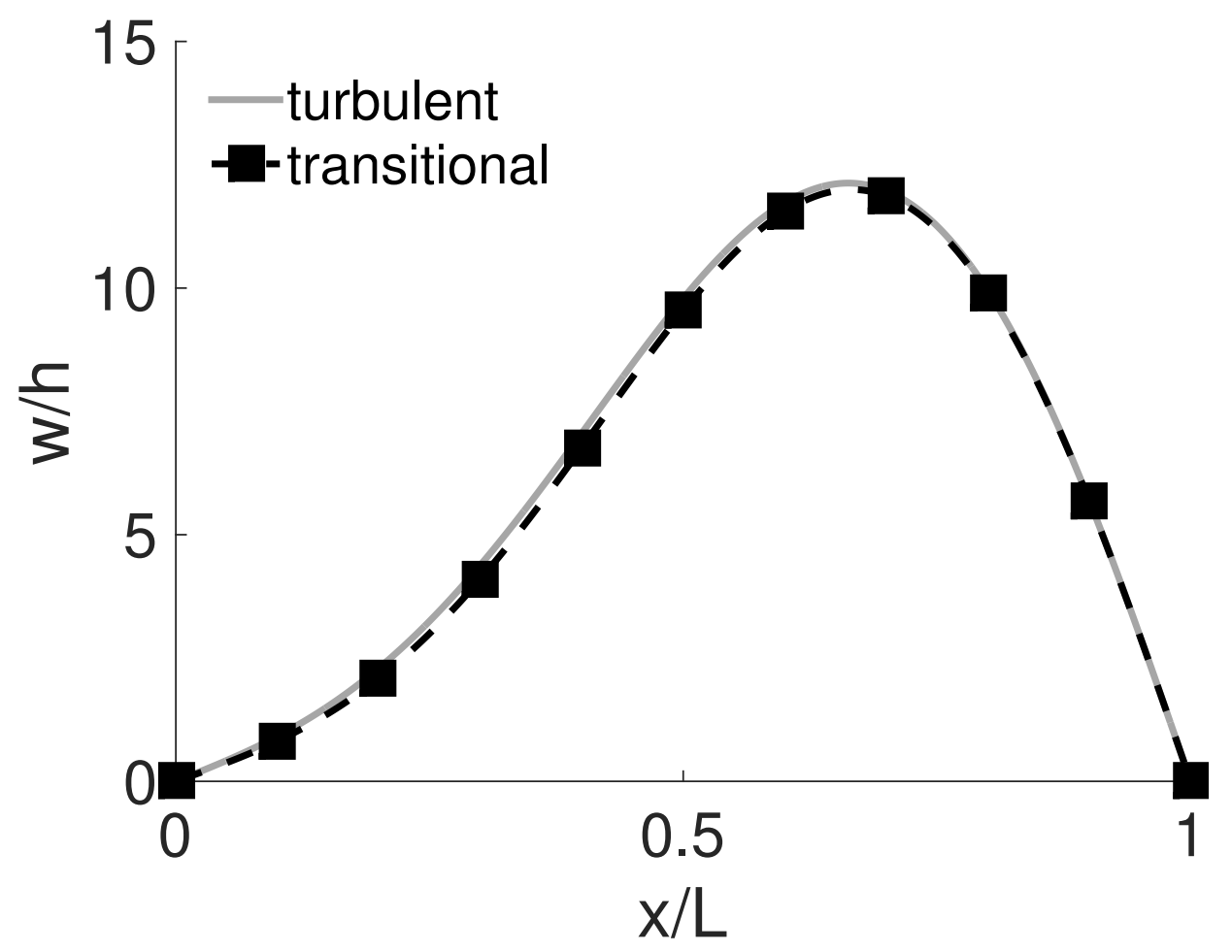

(a) Displacement.

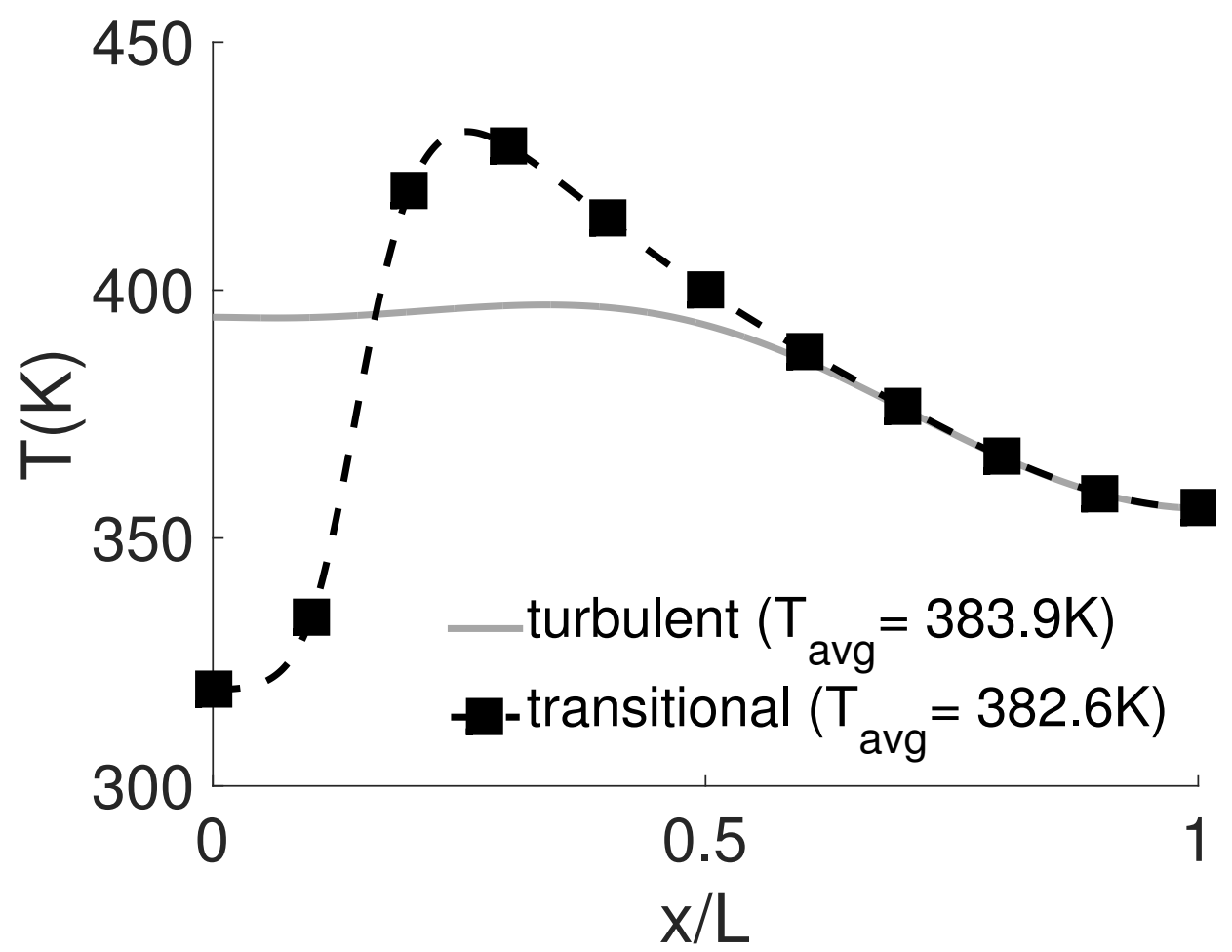

(b) Through-thickness average temperature.

Figure 4.4: Panel response subject to turbulent and transitional $\left(\bar{x}_{t}=0.1, \bar{x}_{t e}=\right.$ 0.25 ) loading with overshoot at $23.0 \mathrm{~s}$. 
corresponding to the most forward and aft transition locations, remain bounded by the laminar and turbulent responses (Figs. 4.5.a and 4.5.b). Additionally, maximum temperatures and thermal gradients are observed to exceed the turbulent values (Figs. 4.5.c and 4.5.d). The responses in Fig. 4.5 indicate that the panel achieves larger deformations and temperatures as transition moves forward on the panel. Interestingly, comparison of Figs. 4.3.a and 4.3.b and Figs. 4.5.a and 4.5.b indicates that variation in the transition onset location produces larger changes in the displacement and slope as compared with variation in the transition length. During the first $23 \mathrm{~s}$ of response, the maximum change in displacement due to altering the transition length is $\Delta w / h=1.6$, whereas shifting the transition location results in a variation of $\Delta w / h=2.5$. A comparison of the maximum temperatures in Figs. 4.3.c and 4.5.c highlights that the maximum variation in temperature due to modifying the transition length $(\Delta T=32 \mathrm{~K})$ is similar to that resulting from altering the transition location $(\Delta T=45 \mathrm{~K})$. However, comparing Figs. 4.3.d and 4.5.d indicates that a variation in transition length $(\Delta d T / d x=854 \mathrm{~K} / \mathrm{m})$ introduces larger changes in the thermal gradient than variation in transition location $(\Delta d T / d x=190 \mathrm{~K} / \mathrm{m})$.

\subsection{Effect of Transition on Flutter Onset}

As another metric of comparison, the impact of the boundary-layer state on the aerothermoelastic stability of the panel is examined. The flutter onset time for 


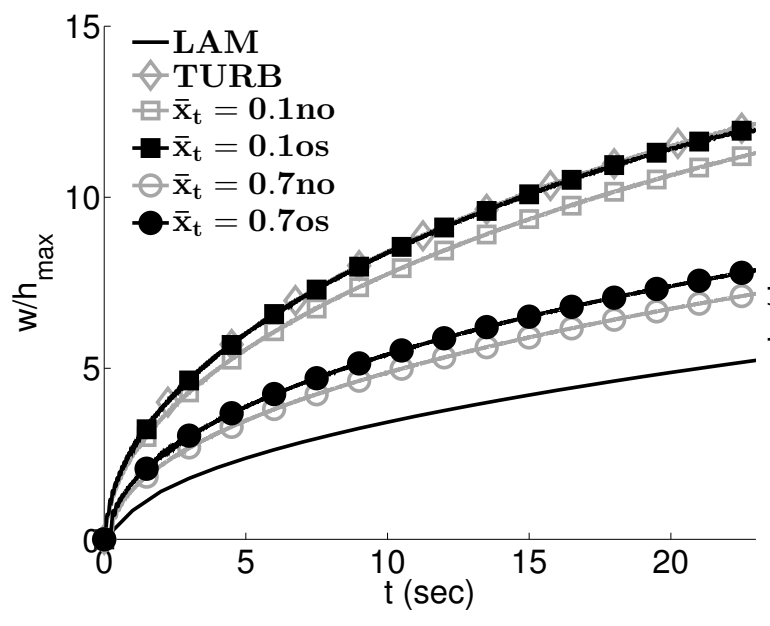

(a) Maximum displacement.

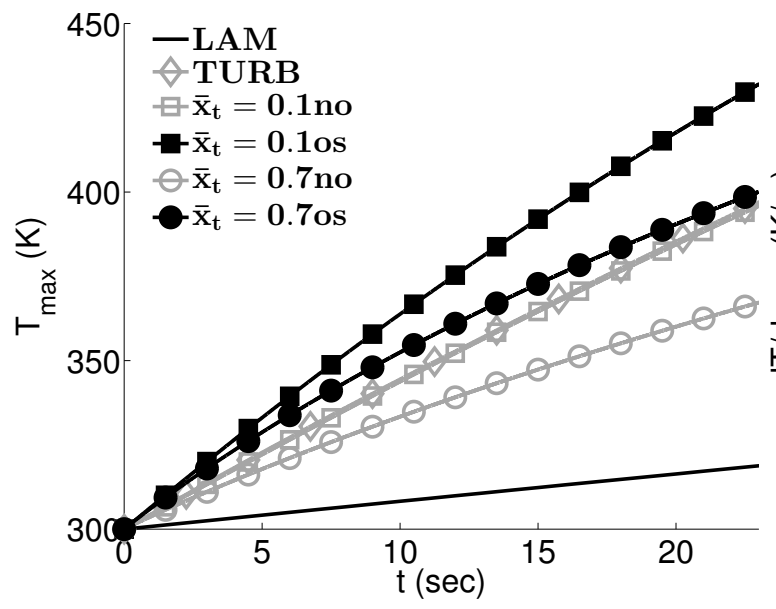

(c) Maximum temperature.

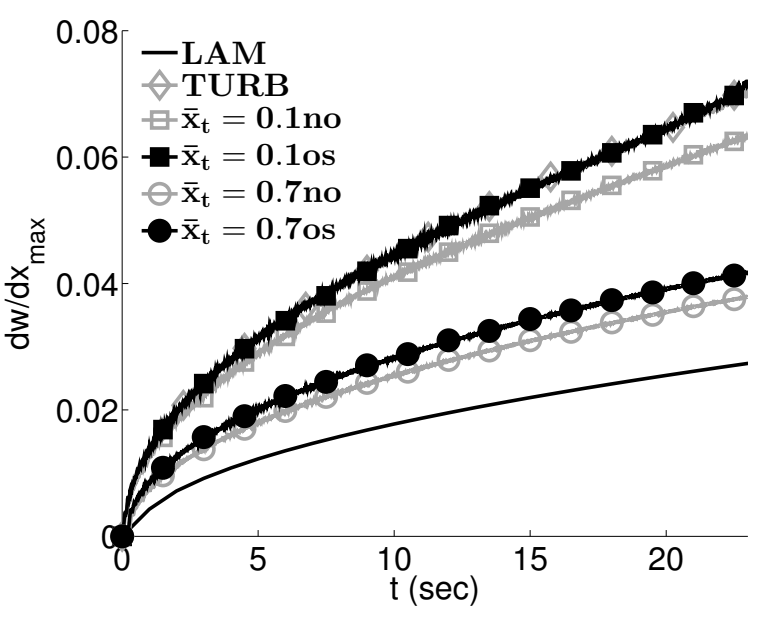

(b) Maximum slope.

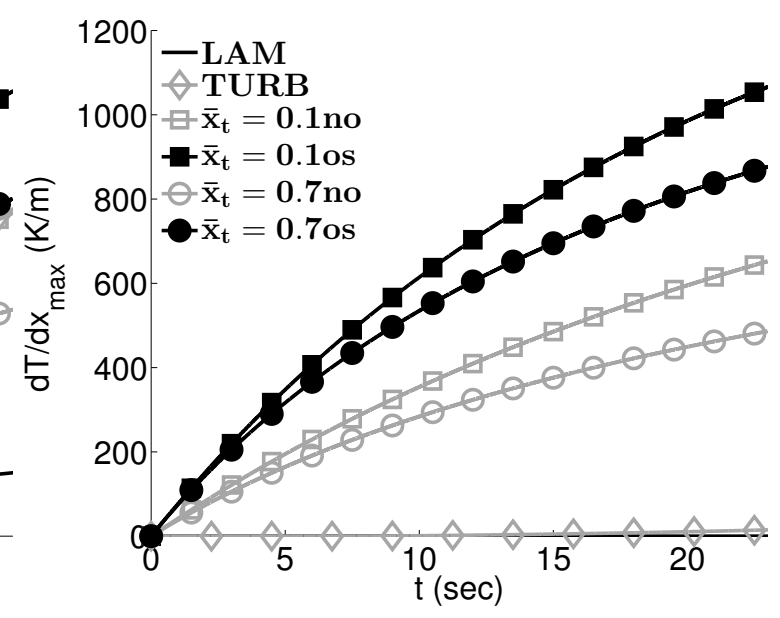

(d) Maximum chordwise temperature gradient.

Figure 4.5: Variation in panel response due to transition location $\left(\Delta \bar{x}_{t}=0.15\right)$ : no overshoot "no" (gray lines and open markers), and overshoot "os" (black lines and closed markers). 
each of the transitional cases considered in this study is shown in Fig. 4.6. The flutter onset time for turbulent flow is also provided as a means to quantify the effect of transitional loading on aerothermoelastic stability. For each transitional case, results are shown corresponding to no overshoot (Fig. 4.6.a) and overshoot (Fig. 4.6.b). For a fixed onset location $\bar{x}_{t}$, moving left to right along the $x$ axis corresponds to increasing the transition length.

Examination of the transitional cases that neglect overshoot (Fig. 4.6.a) indicates that the flutter onset time increases as 1) the transition onset moves downstream, and 2) the transition length increases. This is expected, as either situation implies that the flutter time increases as the turbulent aerothermodynamic loading acts over a smaller region of the panel. However, when overshoot is accounted for (Fig. 4.6.b), the time to flutter does not always increase with transition length. Several transitional configurations $\left(\bar{x}_{t}=0.3,0.5\right)$ in Fig. 4.6.b suggest that the time to flutter is relatively insensitive to variation in the transition length over certain regions of the panel $\left(0.55 \leq \bar{x}_{t e} \leq 0.65\right)$. Furthermore, for $\bar{x}_{t}=0.5$, as the end of transition moves downstream from $\bar{x}_{t e}=0.55-0.65$, the time to flutter decreases. This finding is non-intuitive and is not an aspect of the cases that neglect overshoot.

A comparison of the responses with and without overshoot in Fig. 4.6 reveals that accounting for overshoot always reduces the flutter onset time. As shown in Fig. 4.6.b, the reduction in flutter onset, due to overshoot, causes several transitional loading configurations $\left(\bar{x}_{t}=0.1, \bar{x}_{t e} \leq 0.30\right)$ to flutter earlier than the panel 
subject to fully turbulent loading. The variation in flutter time due to overshoot [defined in Eq. (4.2)] is shown in Fig. 4.7 for each transition onset location. The results in Fig. 4.7 indicate that the difference in flutter time generally increases with the transition length.

$$
\Delta t_{F}=\frac{t_{F_{n o \text { overshoot }}-t_{F_{\text {overshoot }}}}}{t_{F_{\text {turb }}}}
$$

The transitional responses are further characterized in terms of the chordwise average temperature at flutter onset (Fig. 4.8) and the maximum displacement achieved by the panel during the preflutter response (Fig. 4.9). The similarities between Figs. 4.8 and 4.9 indicate that the displacement is driven largely by the average temperature of the panel. Also, from Figs. 4.8.b and 4.9.b, it is apparent that several overshoot configurations exhibit average temperatures $\left(\bar{x}_{t}=0.3,0.40\right.$ $\left.\leq \bar{x}_{t e} \leq 0.55\right)$ and peak displacements $\left(\bar{x}_{t}=0.3,0.35 \leq \bar{x}_{t e} \leq 0.55\right)$ exceeding that produced by turbulent loading.

A comparison of the cases that neglect (Figs. 4.8.a and 4.9.a) or include (Figs. 4.8.b and 4.9.b) overshoot indicates that, at onset locations of $\bar{x}_{t}=0.1-0.5$, accounting for overshoot results in a larger variation in the average temperature and maximum displacement as the transition length changes. The largest variation in temperature $(\Delta T=11 \mathrm{~K})$ and displacement $(\Delta w / h=0.88)$ occurs as the transition length varies for a fixed onset location of $\bar{x}_{t}=0.3$. The increased variation in the temperature and displacement of the panel suggests that transitional overshoot introduces more uncertainty into structural response prediction if the transition length is un- 


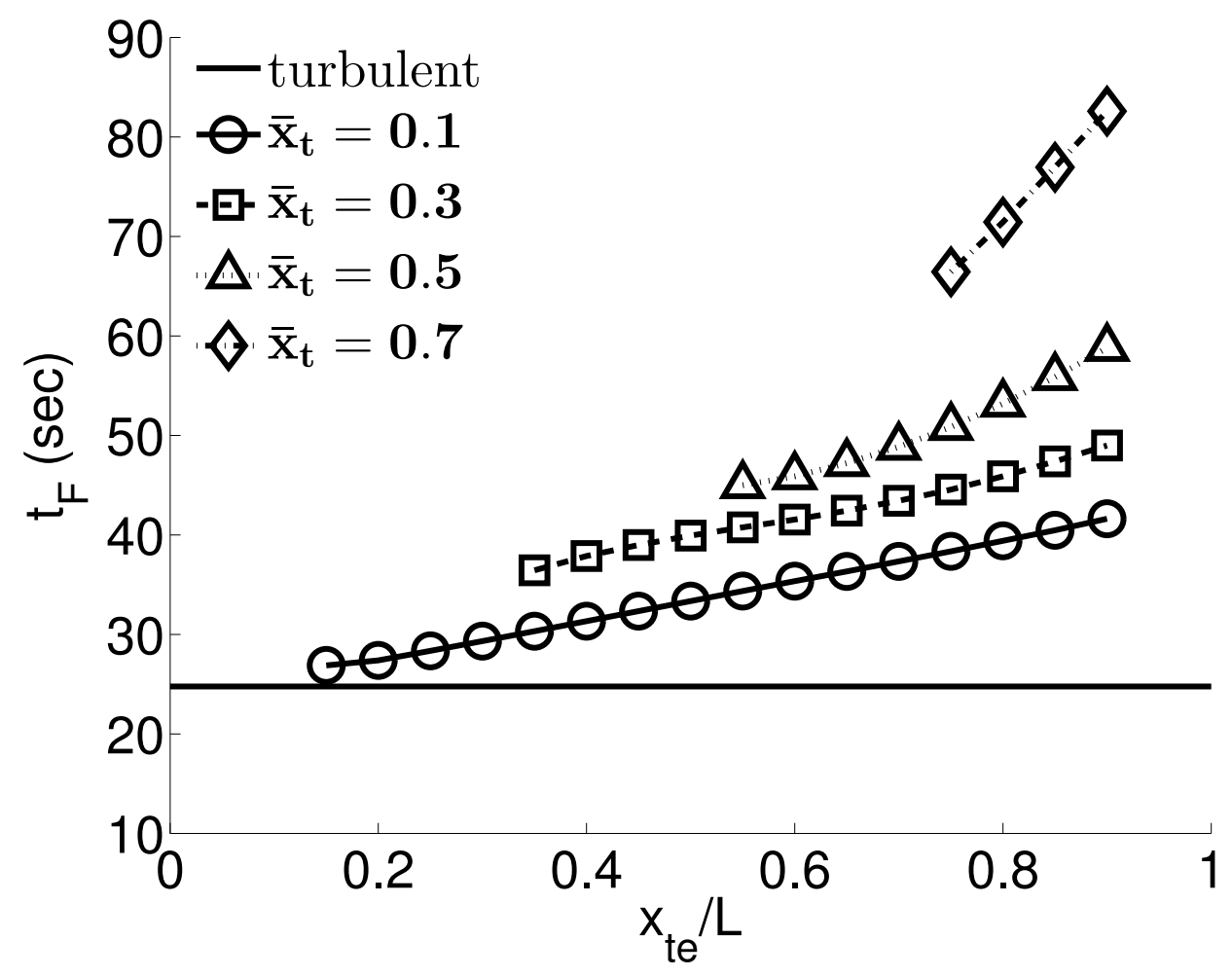

(a) No overshoot.

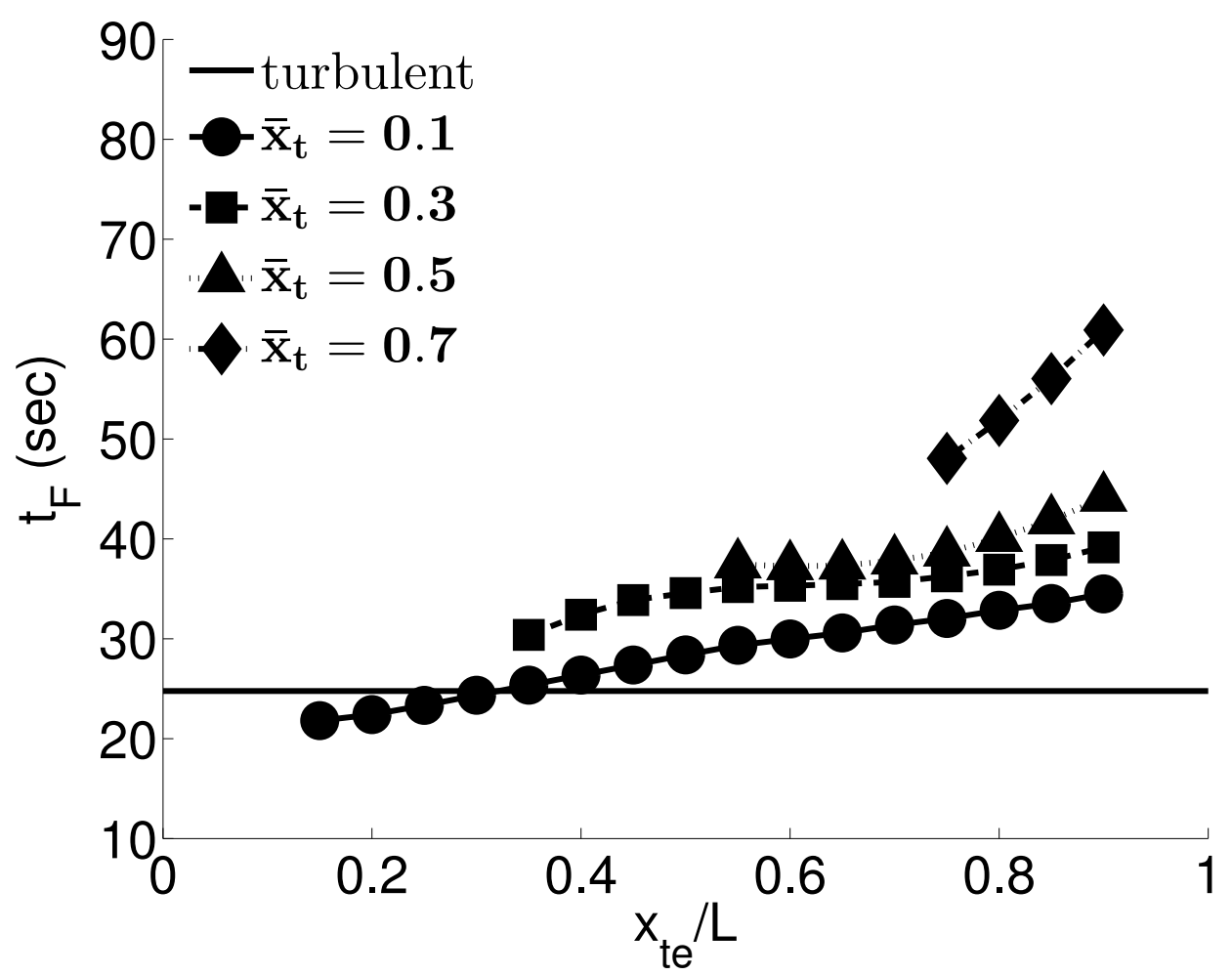

(b) Overshoot.

Figure 4.6: Time to flutter. Laminar flutter condition of $t_{F}=142.5 \mathrm{~s}$ is not shown. 


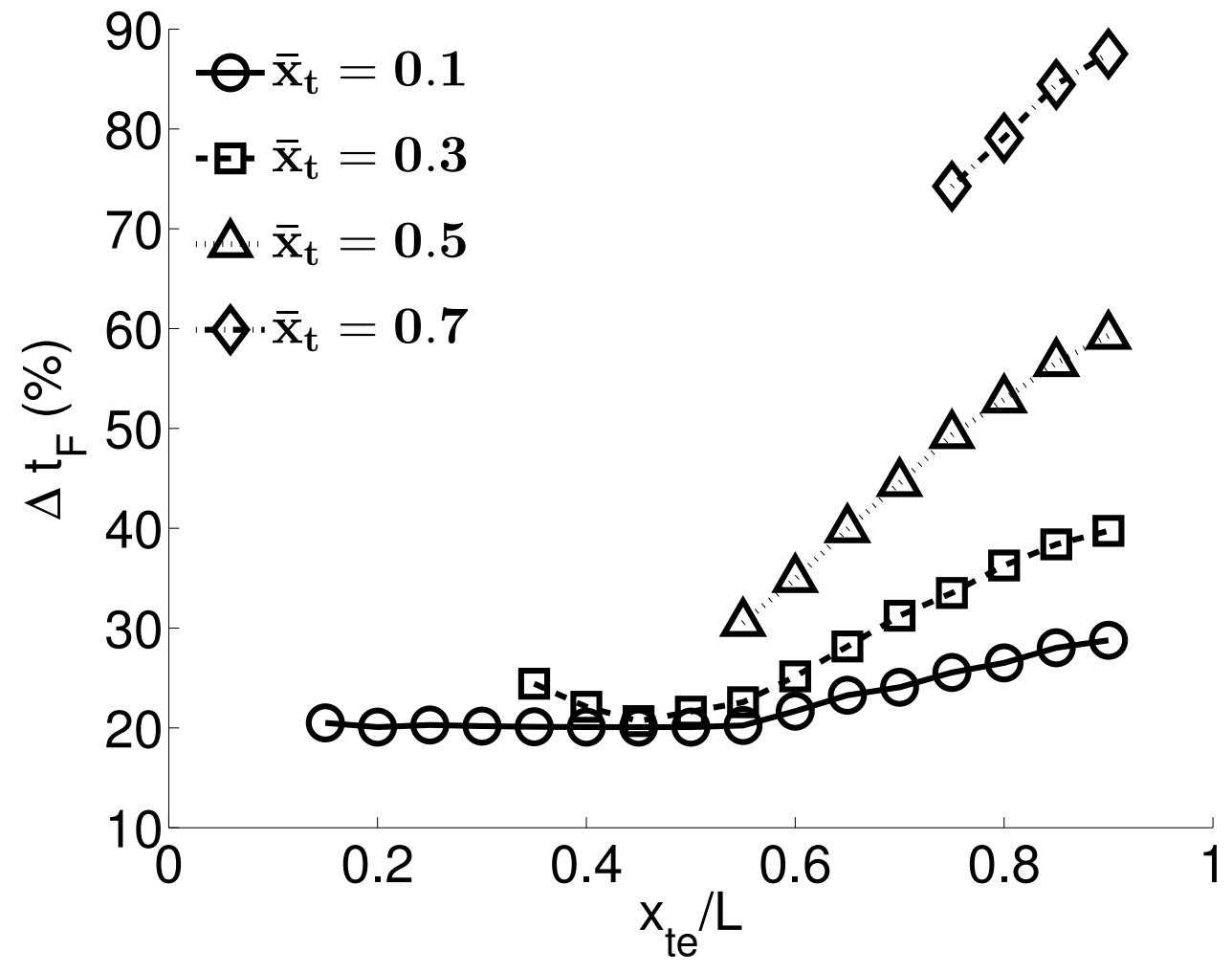

Figure 4.7: Variation in flutter time due to overshoot. 
known.

The overshoot cases in Figs. 4.8.b and 4.9.b illustrate that, for onset at $\bar{x}_{t}=0.5$, as the end of the transition region increases from $\bar{x}_{t e}=0.55$ to 0.80 , both the average temperature and peak displacement decrease. For these configurations, the time to flutter decreases as $\bar{x}_{t e}$ increases from 0.55 to 0.65 . Therefore, for these cases $\left(\bar{x}_{t}=0.5\right.$ and $\left.0.55 \leq \bar{x}_{t e} \leq 0.65\right)$, the panel enters the dynamic instability earlier in the response, even though the component of the inplane thermal force driven by the chordwise average temperature is decreasing. This implies that the reduction in flutter onset is potentially due to the chordwise variation of the temperaturedependent material properties $(E, \alpha)$ resulting from the transitional heat flux distribution.

\subsection{Effect of Temperature-Dependent Material Proper- ties}

Further investigation into the reduction in flutter time, for the overshoot cases shown in Fig. 4.6.b, is provided next for several cases $\left(\bar{x}_{t}=0.5, \bar{x}_{t e}=0.55,0.60\right.$, and 0.65). For each case, the through-thickness average temperature along the panel at the time of flutter is shown in Fig. 4.10. The times listed in Fig. 4.10 correspond to flutter onset for each response. Note that the peak temperature occurs near the end of the transition region. A comparison of the profiles in Fig. 4.10 reveals that, as $\bar{x}_{t e}$ 


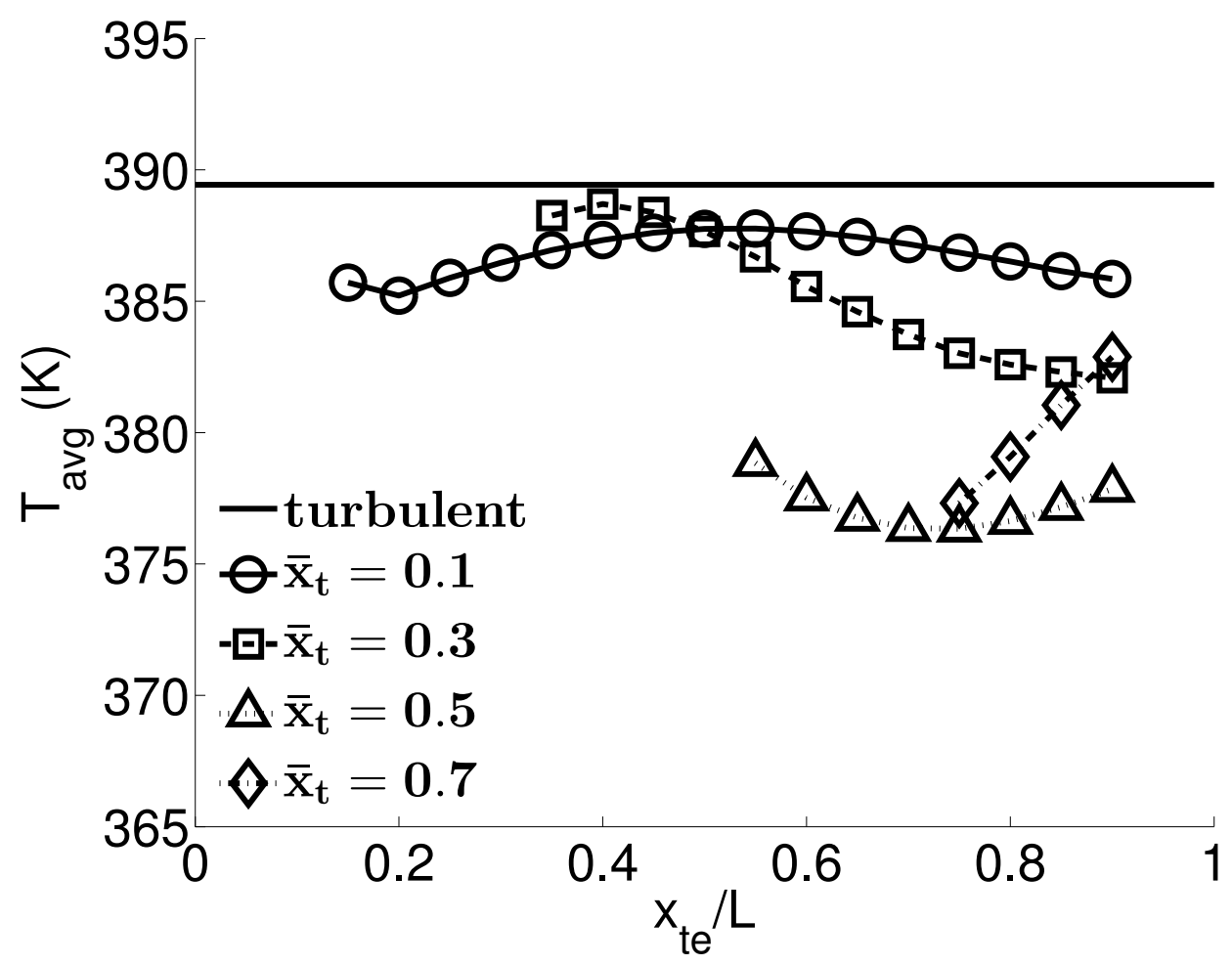

(a) No overshoot.

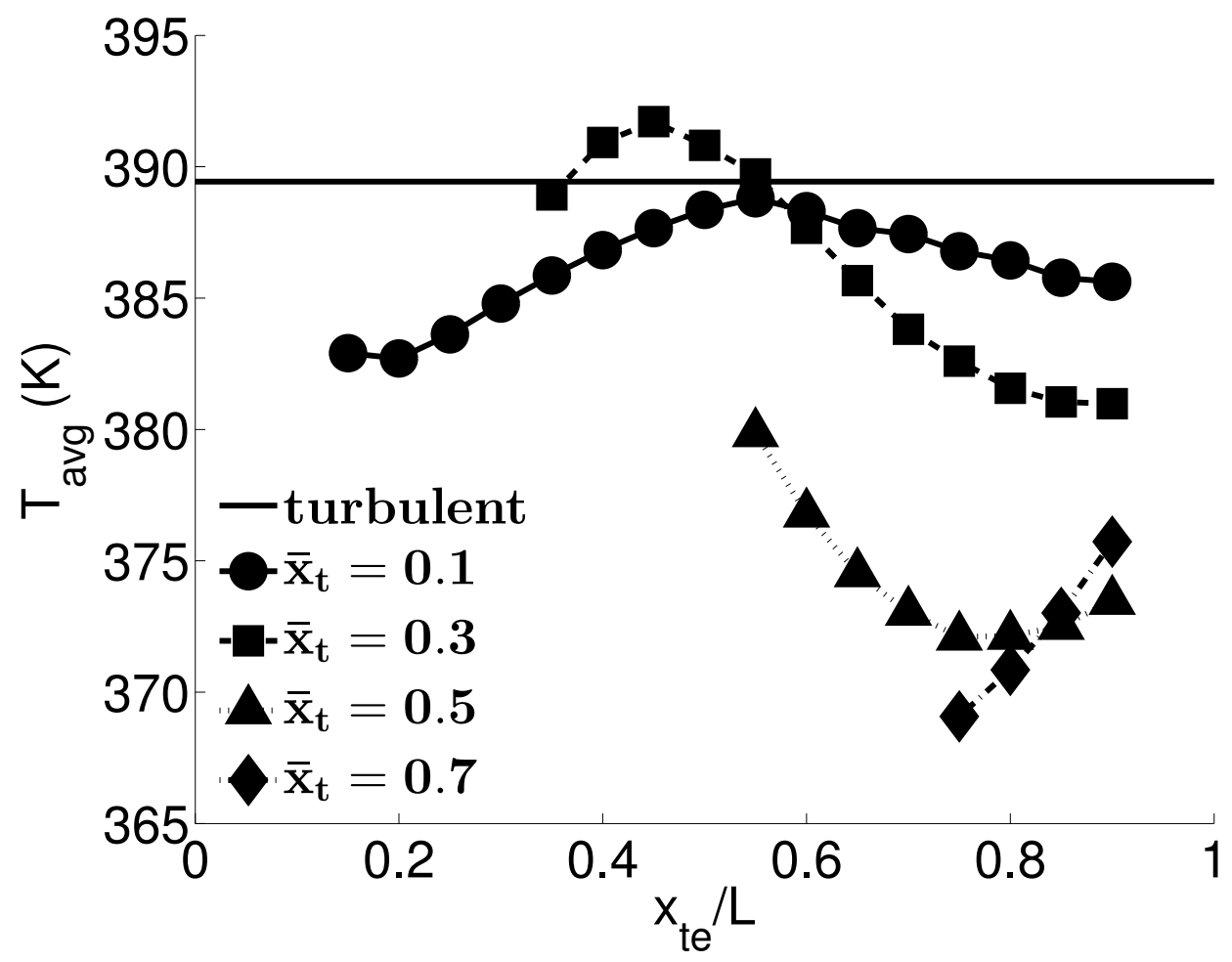

(b) Overshoot.

Figure 4.8: Chordwise average temperature at flutter. 


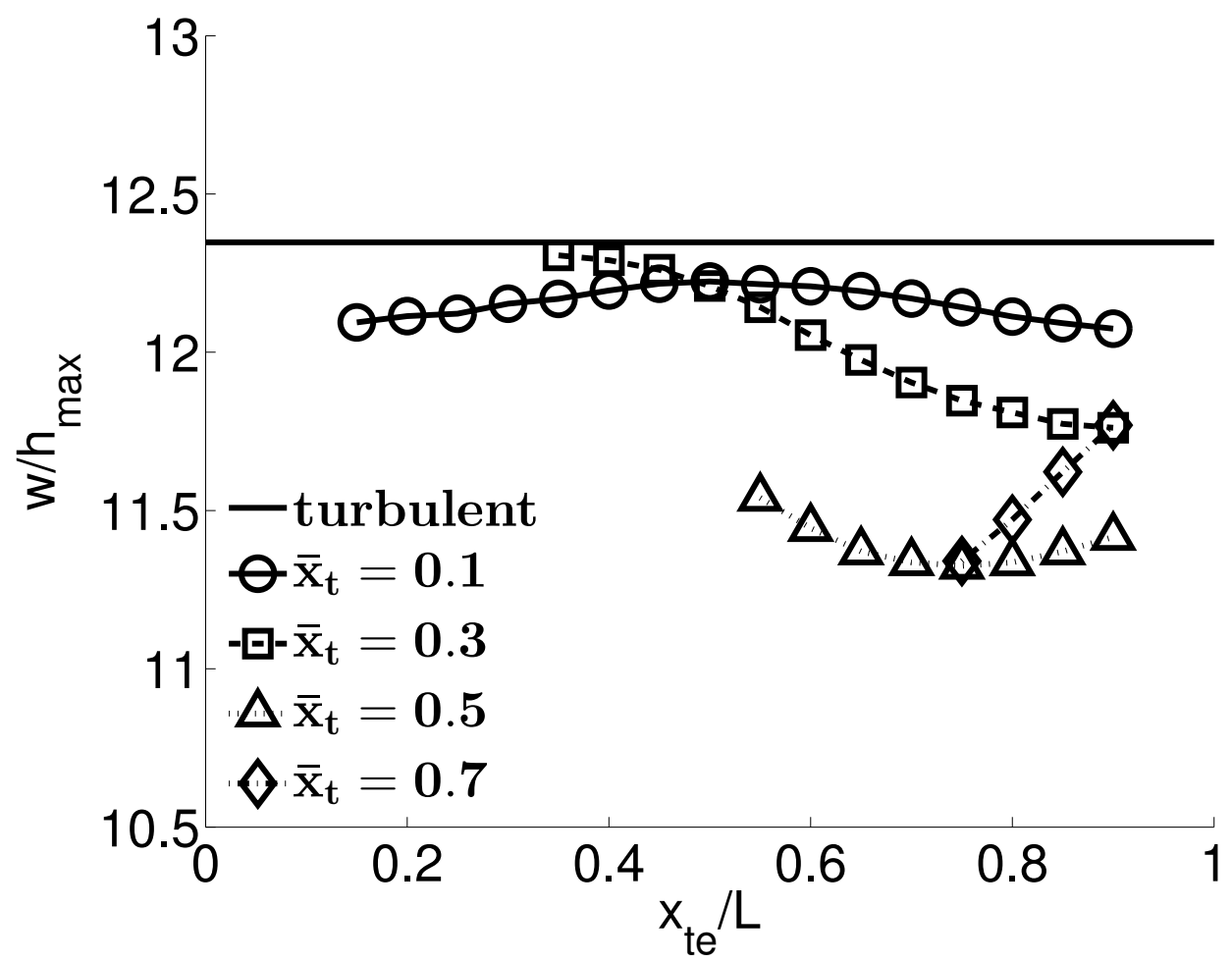

(a) No overshoot.

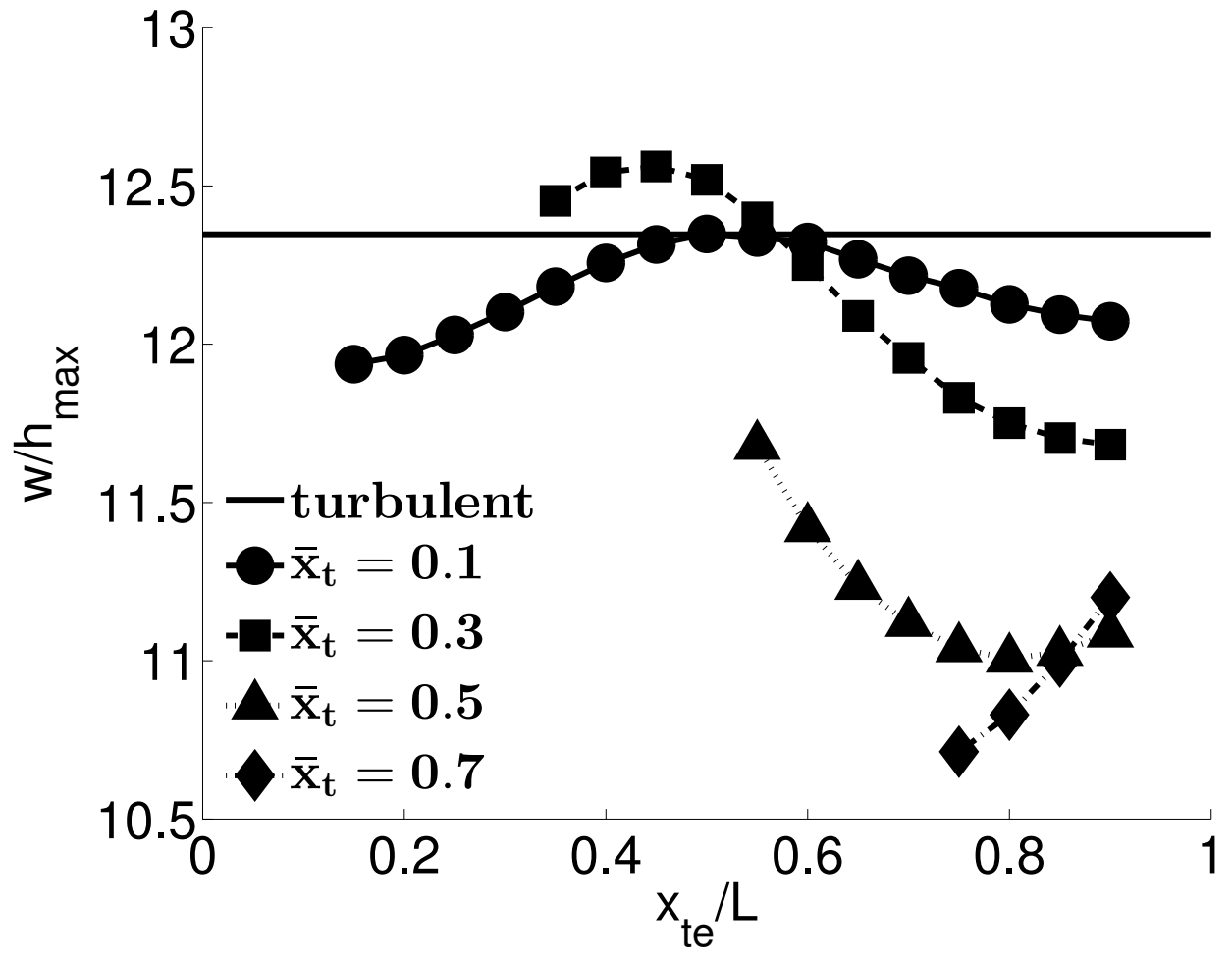

(b) Overshoot.

Figure 4.9: Maximum displacement before flutter. 


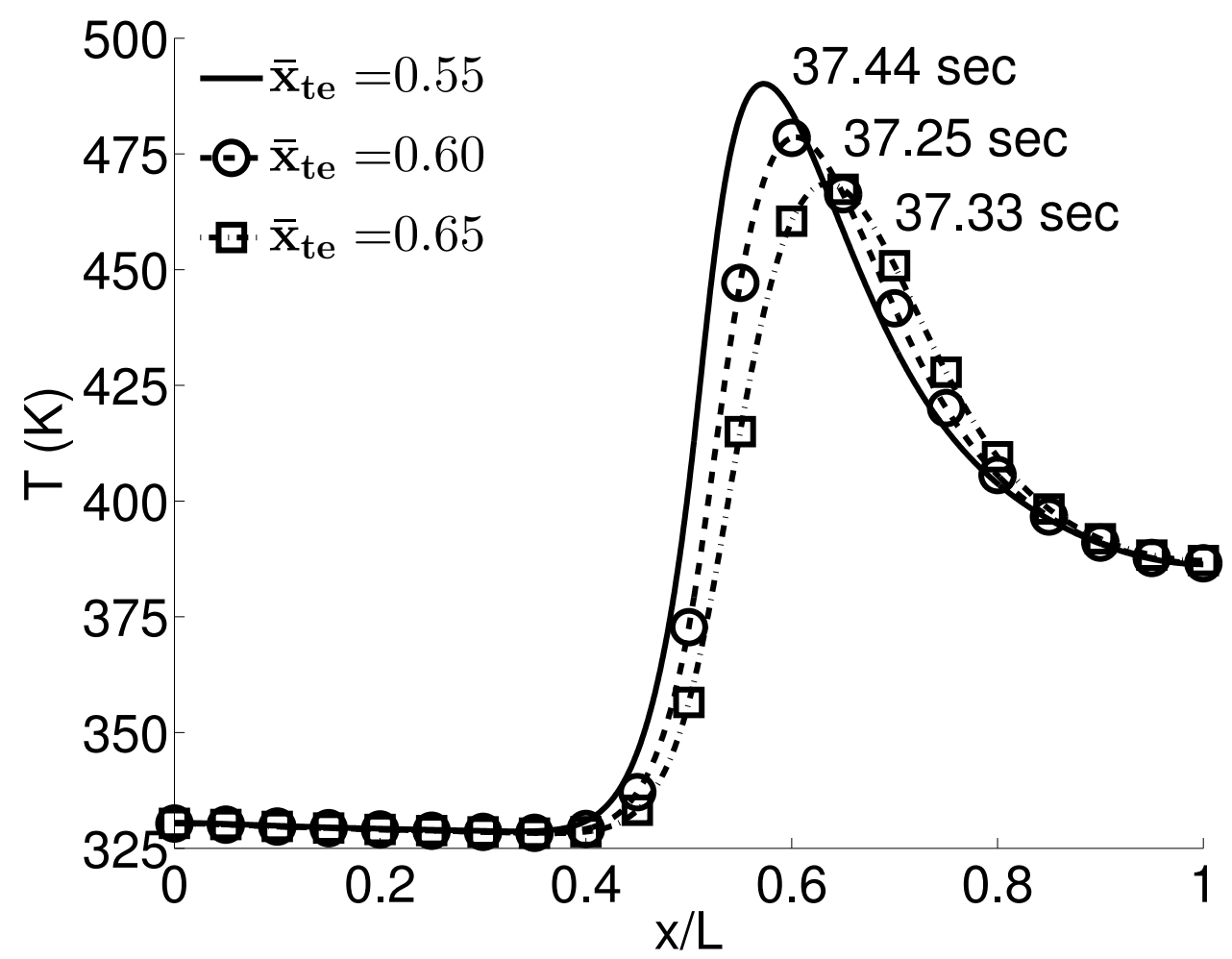

Figure 4.10: Through-thickness average temperature profile at flutter onset.

moves downstream, the through-thickness average temperature decreases for $\bar{x} \leq$ 0.614 and increases on the trailing edge of the panel $(\bar{x} \geq 0.647)$.

The impact of the chordwise thermal gradient on the material properties of the panel is shown in Fig. 4.11, in terms of the modulus of elasticity and thermal expansion coefficient at the time of flutter. A comparison of the profiles in Fig. 4.11 indicates that, for $\bar{x} \leq 0.614$, as $\bar{x}_{t e}$ moves rearward along the panel the modulus of elasticity increases (Fig. 4.11.a) and the thermal expansion coefficient decreases (Fig. 4.11.b). Conversely, on the trailing edge of the panel ( $\bar{x} \geq 0.647)$, as $\bar{x}_{t e}$ moves rearward, the modulus of elasticity decreases (Fig. 4.11.a) and the thermal expansion coefficient increases (Fig. 4.11.b). Therefore, as the length of the transition 
region increases, the forward portion of the panel $(\bar{x} \leq 0.614)$ stiffens while the aft region $(\bar{x} \geq 0.647)$ becomes more prone to deformation. A primary driver of the panel response is the in-plane thermal load which, as previously mentioned, is primarily dependent on both the chordwise average temperature and temperaturedependent material properties [35]. As shown in Fig. 4.8.b, the contribution of the average temperature to the in-plane thermal effects is decreasing. Therefore, the reduction in flutter time is presumably due in part to the large spatial variation in the material properties shown in Fig. 4.11.

The effect of the temperature-dependent material properties on the flutter onset time is depicted in Fig. 4.12, where each line corresponds to a transition onset of $\bar{x}_{t}=0.5$. The curves labeled " $f(T)$ " and "const" identify responses obtained with either temperature-dependent or constant material properties. The constant material properties correspond to those listed in Table 4.3 for a temperature of 300 K. For each case, results are presented for transitional responses that either include or neglect overshoot. The results in Fig. 4.12 indicate that the flutter time increases when assuming constant material properties. Additionally, the localized reduction in flutter time, which occurs for the temperature-dependent overshoot configuration with $\bar{x}_{t e}=0.60$, is no longer present when assuming constant material properties. 


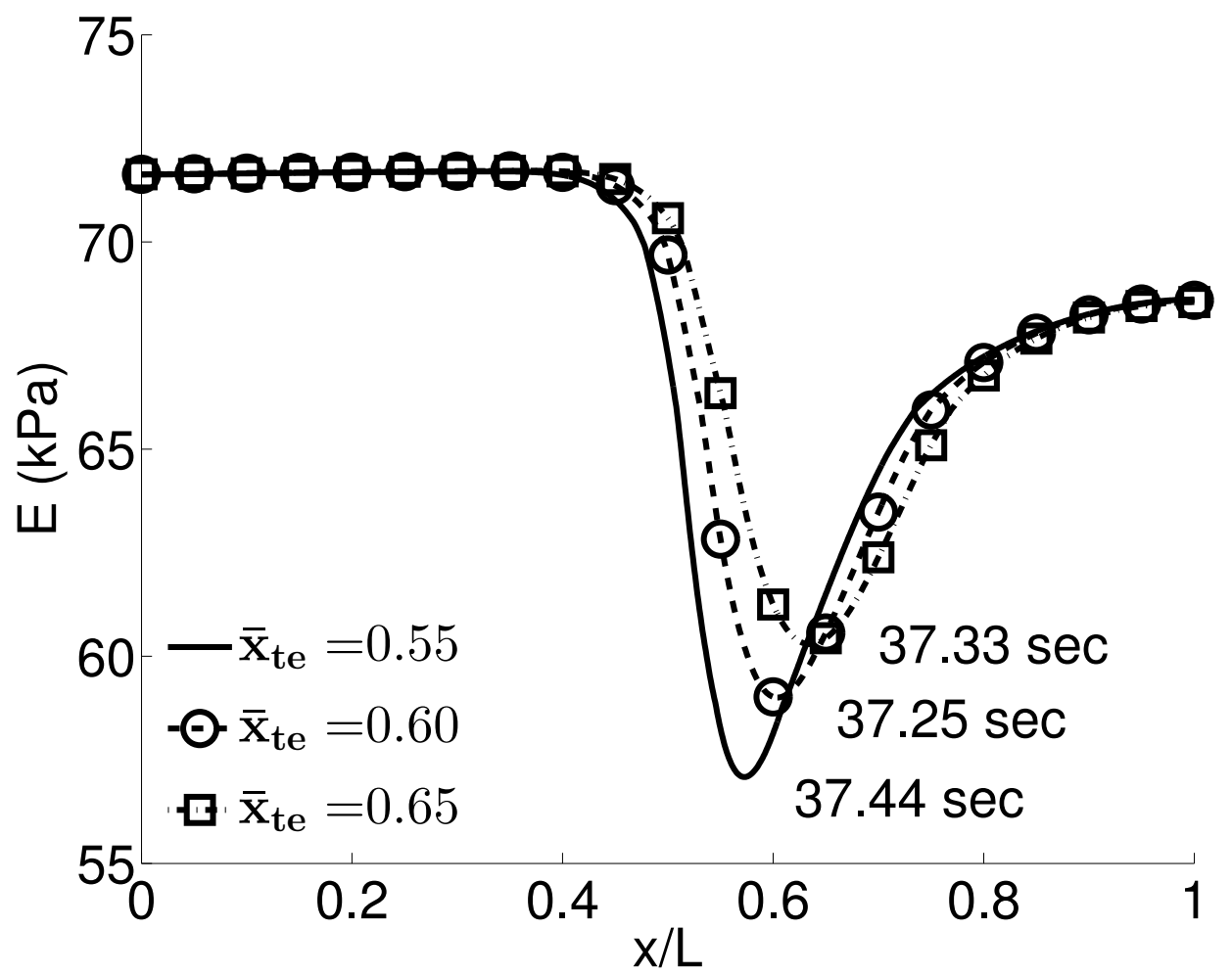

(a) Modulus of elasticity.

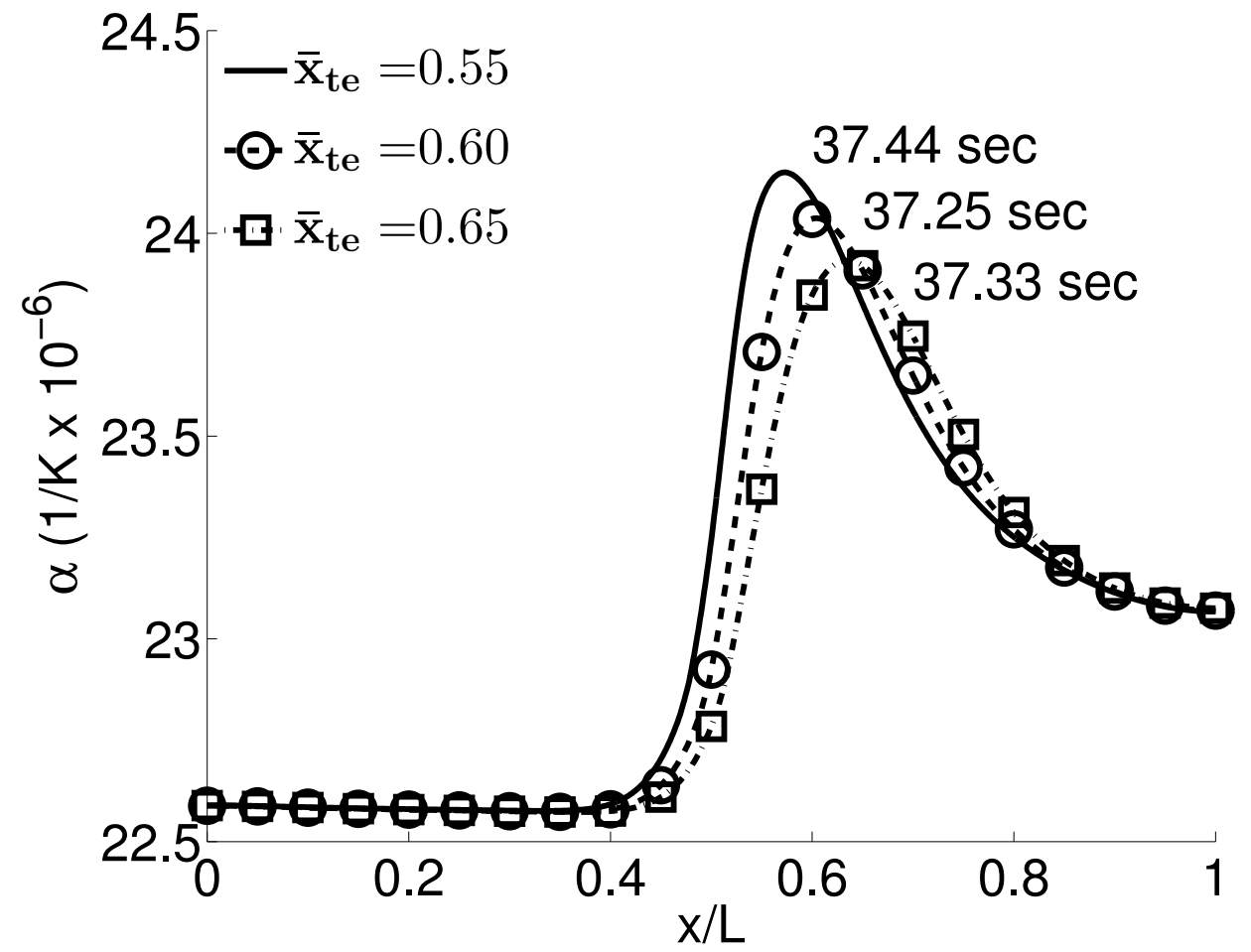

(b) Thermal expansion coefficient.

Figure 4.11: Temperature-dependent material properties at flutter onset. 


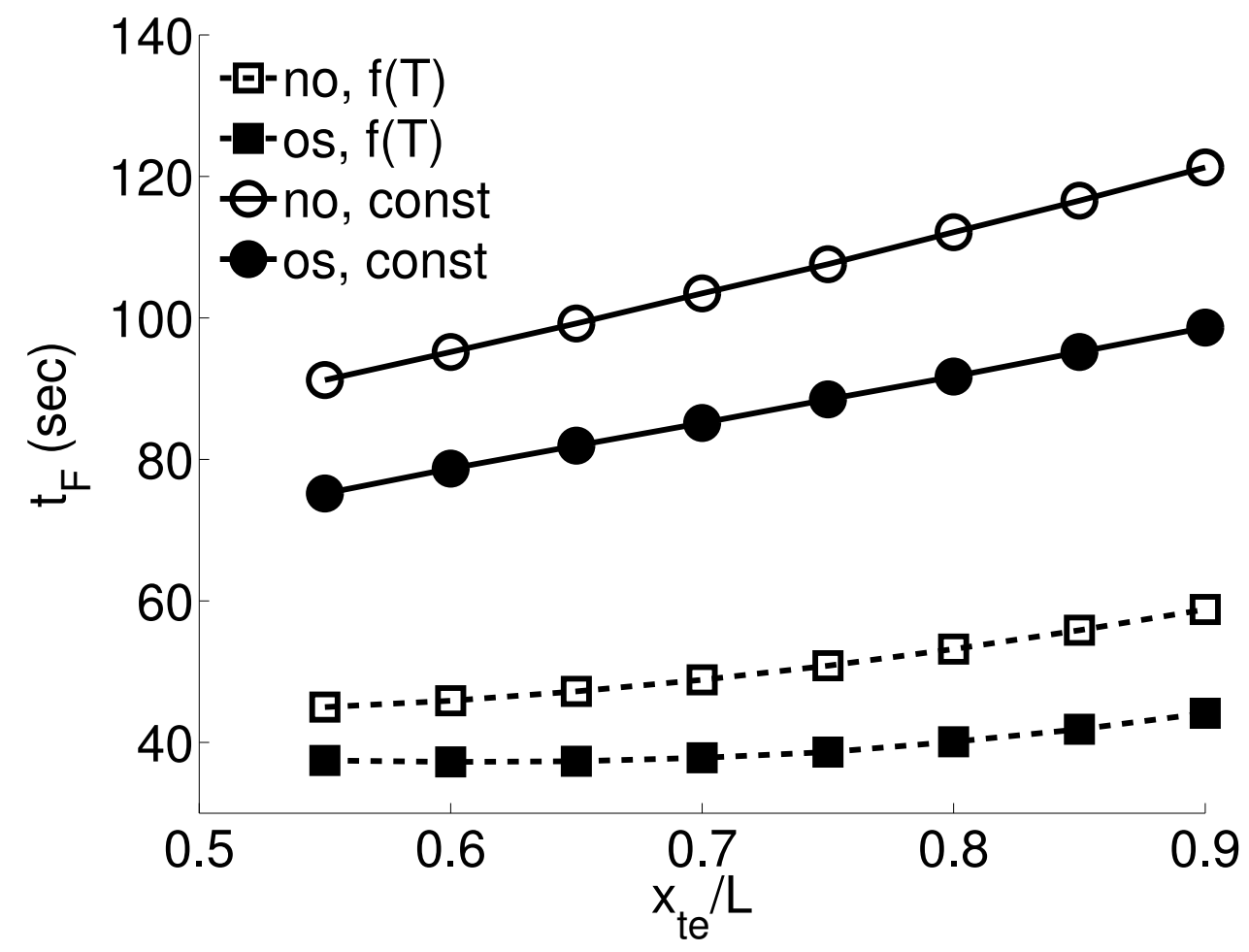

Figure 4.12: Effect of temperature dependence on flutter time: no overshoot "no" (open markers), and overshoot "os" (closed markers). 


\subsection{Effect of Transition on Panel Life Considerations}

The time-integrated strain energy is used as a metric to assess the impact of transition on panel life. This quantity is chosen because it has been found to correlate with fatigue life in metallic coupons [161]. The strain energy accumulated in the panel, during the first $20 \mathrm{~s}$ of response, is shown in Fig. 4.13 for transitional loading configurations that neglect (Fig. 4.13.a) or account for (Fig. 4.13.b) overshoot. Examination of Fig. 4.13 indicates that the strain energy accumulated in the panel decreases as the transition onset moves downstream and as the length of the transition region increases. Furthermore, for a fixed transition onset and length, accounting for overshoot increases the accumulated strain energy. As shown in Fig. 4.13.b, several transitional cases $\left(\bar{x}_{t}=0.1, \bar{x}_{t e} \leq 0.3\right)$ result in strain energy accumulation that exceeds that produced by turbulent loading. Previous studies have found that failure occurs once a critical level of strain energy is accumulated in the structure $[161,162]$. Therefore, the results in Fig. 4.13.b suggest that turbulent loading may over predict the life of the structure if transition is likely to occur. 


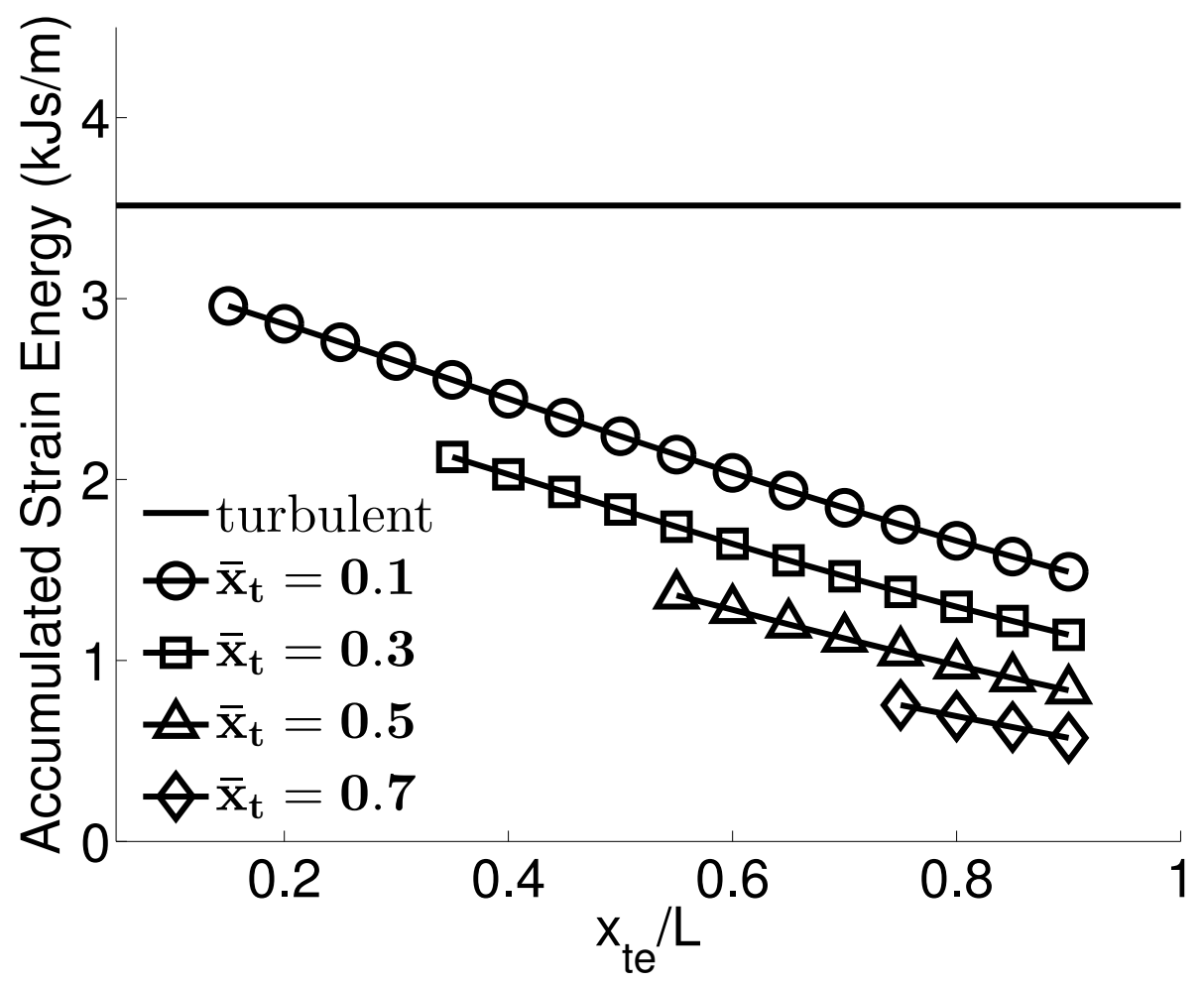

(a) No overshoot.

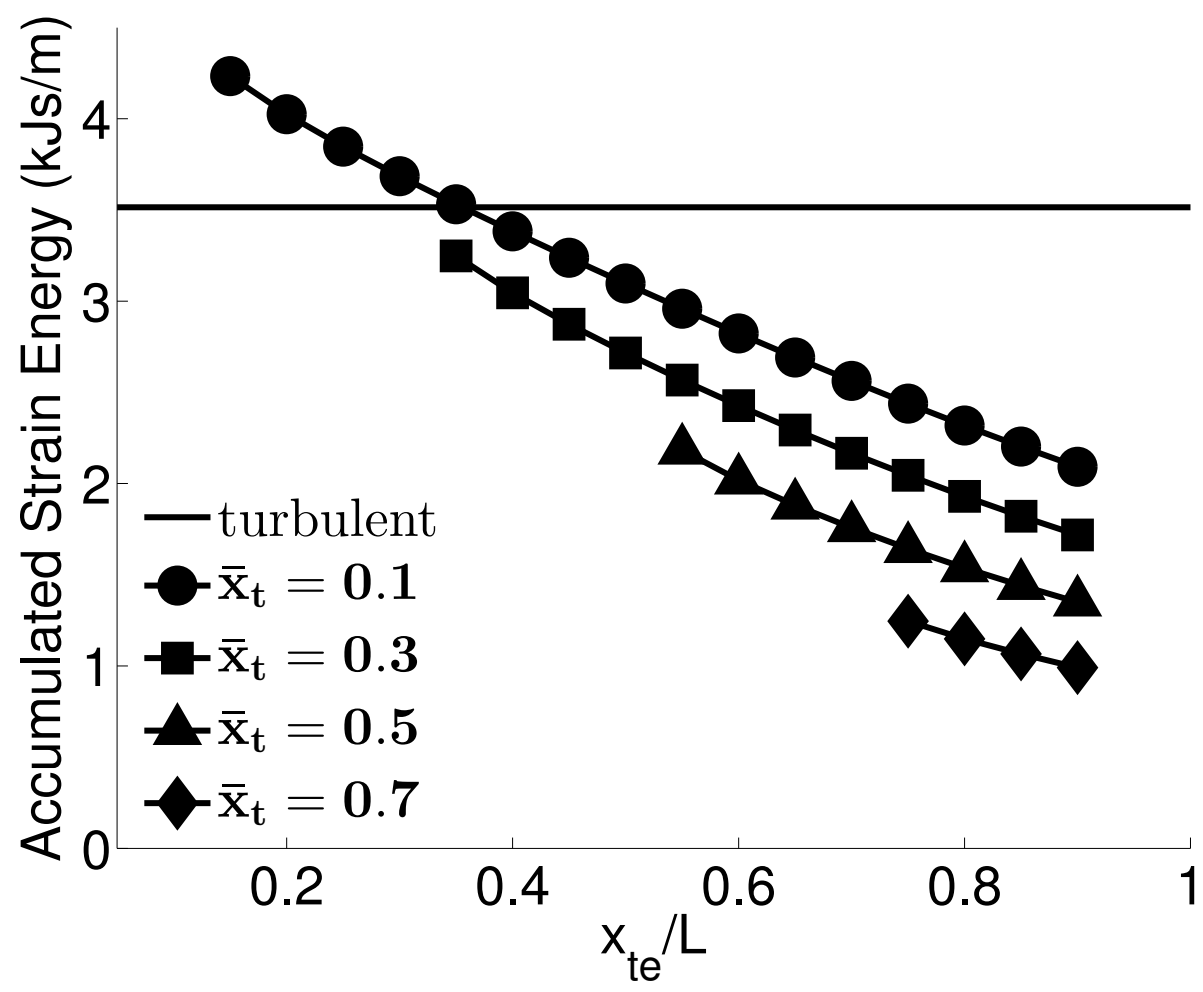

(b) Overshoot.

Figure 4.13: Strain energy accumulation during the first $20 \mathrm{~s}$ of response. 


\section{Chapter 5}

\section{Interaction between Boundary-Layer}

\section{Transition and Structural Response}

The interaction between boundary-layer transition and the aerothermoelastic response of a representative hypersonic vehicle panel is investigated through comparison of panel responses obtained assuming fully turbulent and transitional fluid loading, where the transition region either remains fixed (as in Chapter 4) or varies in time due to the panel response. The transitional loading assumes overshoot in the heat flux and fluctuating pressure load. The transition onset location is predicted using either STABL (discussed in Section 2.1) or a surrogate model of STABL, which is described in detail in Section 5.2. The transitional cases assume a constant transition length of $0.3 \mathrm{~m}$ and an initial onset location of $2.67 \mathrm{~m}$, which corresponds to the surrogate prediction for the undeformed, stress free (300 K) panel. The freestream conditions and panel configuration are provided in Section 5.1. Results are presented in Section 5.3 for the first $10 \mathrm{~s}$ of response of a titanium panel 
with either simply-supported or clamped structural boundary conditions.

\subsection{Problem Description}

A schematic of the configuration examined here is provided in Fig. 2.15. It is assumed that a titanium panel is located $2 \mathrm{~m}$ from the leading edge of a 2-D surface inclined $5^{\circ}$ to the freestream, representing the forebody of a supersonic/hypersonic vehicle. Both pinned and clamped structural boundary conditions are considered. The freestream conditions and panel geometry are listed in Tables 5.1 and 5.2. The freestream conditions coincide with the proposed cruise velocity and altitude specified for the conceptual Mach 5 to 7 hypersonic cruise vehicle outlined in [11]. Based off the surface temperatures predicted at this cruise condition, titanium alloys could be employed as the outer mold line structure of the vehicle [11]. The mass and stiffness-proportional Rayleigh damping is specified such that $2 \%$ of the critical damping is achieved at both the fundamental natural frequency and at $1000 \mathrm{~Hz}$ to provide viscous damping of the higher frequencies. The temperaturedependent material properties of the titanium panel are listed in Table 5.3. The density, Poisson's ratio, and emissivity [163] are assumed to be constant over the operational range of temperature. The maximum modal frequency of the panel is $3.32 \mathrm{kHz}$ and $3.46 \mathrm{kHz}$ for pinned and clamped structural boundary conditions, respectively. 
Table 5.1: Freestream conditions.

\begin{tabular}{lc}
\hline \hline Parameter & Value \\
\hline Mach number (freestream) & 5.20 \\
Mach number (post oblique shock) & 4.66 \\
Altitude, km & 27.4 \\
Unit Reynolds number, $\times 10^{6} \mathrm{~m}^{-1}$ & 2.85 \\
\hline \hline
\end{tabular}

Table 5.2: Panel geometry.

\begin{tabular}{lc}
\hline \hline Parameter & Value \\
\hline Leading edge location, $\mathrm{m}$ & 2.00 \\
Length, $\mathrm{m}$ & 1.50 \\
Thickness, $\mathrm{mm}$ & 5.00 \\
Density, $\mathrm{kg} / \mathrm{m}^{3}$ & 4540 \\
Poisson's ratio & 0.320 \\
Emissivity & 0.6 \\
\hline \hline
\end{tabular}

Table 5.3: Temperature dependence of material properties of Titanium (Ti-6Al-2Sn-4Zr-2Mo).

\begin{tabular}{ccccc}
\hline \hline$T$ & $c$ & $k$ & $E$ & $\alpha$ \\
$\mathrm{K}$ & $\mathrm{J} / \mathrm{kg} \mathrm{K}$ & $\mathrm{W} / \mathrm{m} \mathrm{K}$ & $\mathrm{GPa}$ & $\mu \mathrm{m} / \mathrm{mK}$ \\
\hline 300 & 463.08 & 6.89 & 113.46 & 7.09 \\
400 & 488.20 & 8.03 & 107.51 & 8.06 \\
500 & 535.10 & 9.28 & 102.39 & 8.78 \\
600 & 593.72 & 10.52 & 97.57 & 9.22 \\
700 & 661.55 & 11.77 & 92.15 & 9.54 \\
800 & 740.26 & 13.17 & 84.01 & 9.70 \\
\hline \hline
\end{tabular}

\subsection{Transition Onset Surrogate Model}

A kriging surrogate model is generated in order to reduce the online computational expense associated with prediction of the transition onset location within an aerothermoelastic response simulation. Kriging is an interpolation method that is well suited to approximate nonlinear behavior and does not require a priori knowl- 
edge of the full-order relationship [103]. The parameter space for the surrogate includes an isothermal surface temperature and the deformation of the panel, approximated using the computed amplitudes of the first 3 free vibration modes. Approximating the deformation in this manner greatly reduces the transition onset surrogate parameter space while producing a maximum error of just $0.6 h$. Note that the peak deformation of the panel is on the order of $4 h$. The output for the surrogate is the maximum $N$ factor envelope.

Training points are chosen using a quasi-random, Halton sequence sampling of the parameter space. Generation of the surrogate training points requires both a CFD solution and a corresponding stability analysis. Specifically, for each training point STABL is used to: (1) generate a fluid mesh that accounts for the panel deformation; (2) perform a CFD analysis at the specified wall temperature; (3) assess the boundary-layer stability using LPSE; and (4) obtain the maximum $N$ factor envelope for a range of disturbance frequencies. Using the training points, a kriging

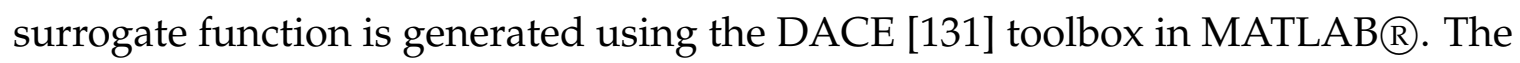
surrogate function predicts the maximum $N$ factor curve based off the input wall temperature and surface deformation.

\subsubsection{Mean Flow and LPSE Grid Convergence}

A grid resolution study is performed to determine the required mean flow and LPSE test matrix mesh densities that result in converged maximum $N$ factor curves. 


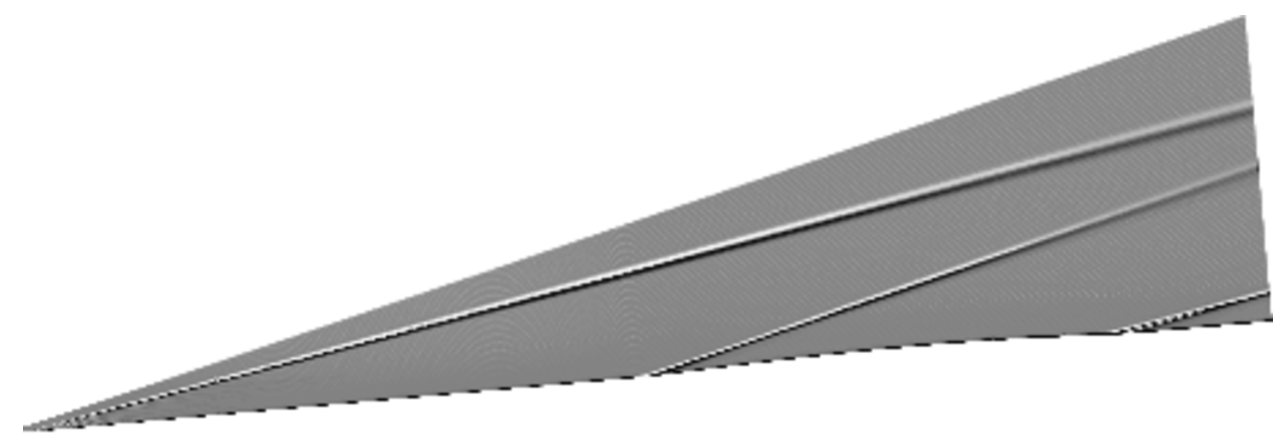

Figure 5.1: Shadowgraph of flowfield $\left(a_{1} / h=4.204, a_{2} / h=-0.374, a_{3} / h=0.085\right)$.

For each training point, the computational domain resembles that shown in Fig. 5.1.

Derivatives of the wall-normal velocity and temperature, at the center of the panel, are shown in Fig. 5.2 corresponding to the panel deformation in Fig. 5.1. A comparison of the results in Fig. 5.2 indicate that grid independence is achieved with 400 surface normal grid points. The resolved grid has a maximum cell spacing along the surface of $2 \mathrm{~mm}$, a minimum of 118 cells defining the boundary layer, and a $y^{+}$value of less than 0.3 in the first cell from the surface. Grid independence of the stability analysis is demonstrated with respect to the mean flow mesh density in Fig. 5.3.a and the LPSE test matrix (surface distance and frequency) density in Fig. 5.3.b.

\subsubsection{Surrogate Generation}

Bounds for the surrogate input parameters are specified to include the predicted surface temperatures and deformation modal weights during the first $10 \mathrm{~s}$ of response for the simply-supported panel subject to fully turbulent and prescribed transitional loading. A safety margin of $\pm 20 \%$ of the parameter range is added to 


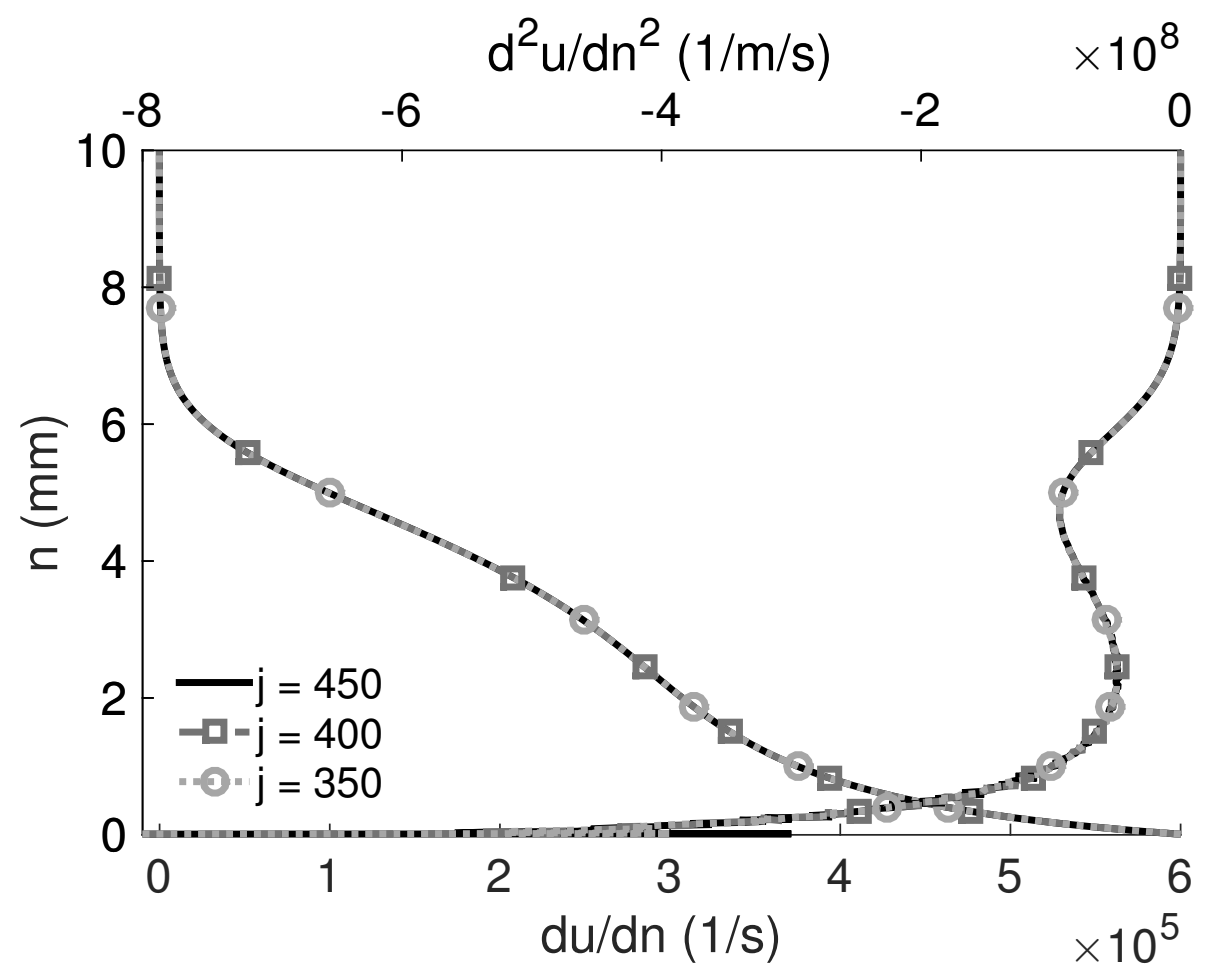

(a) Velocity.

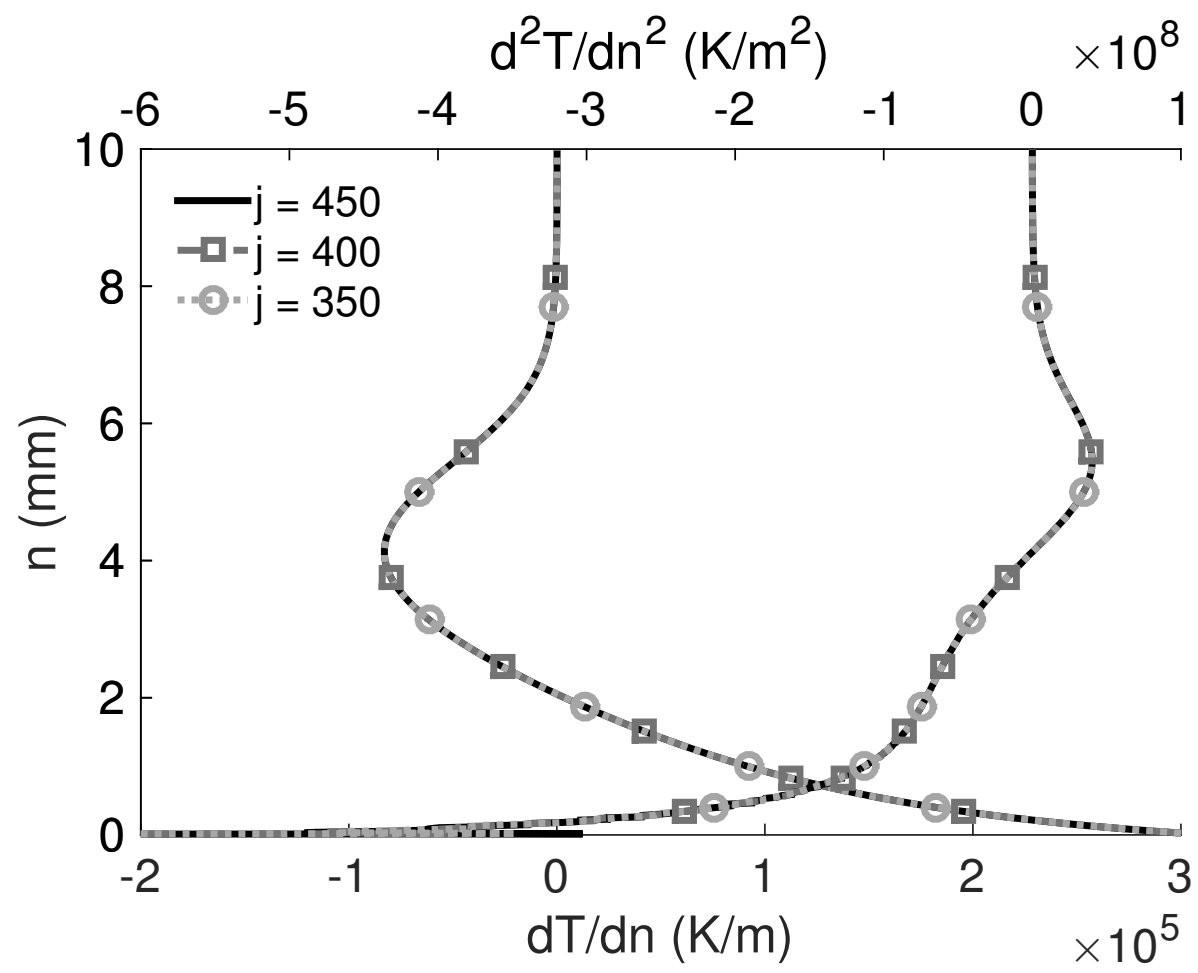

(b) Temperature.

Figure 5.2: Wall normal derivatives at midpoint of panel $S=2.75 \mathrm{~m}\left(T_{w}=300 \mathrm{~K}\right.$, $\left.a_{1-3} / h=4.204,-0.374,0.085\right)$. 


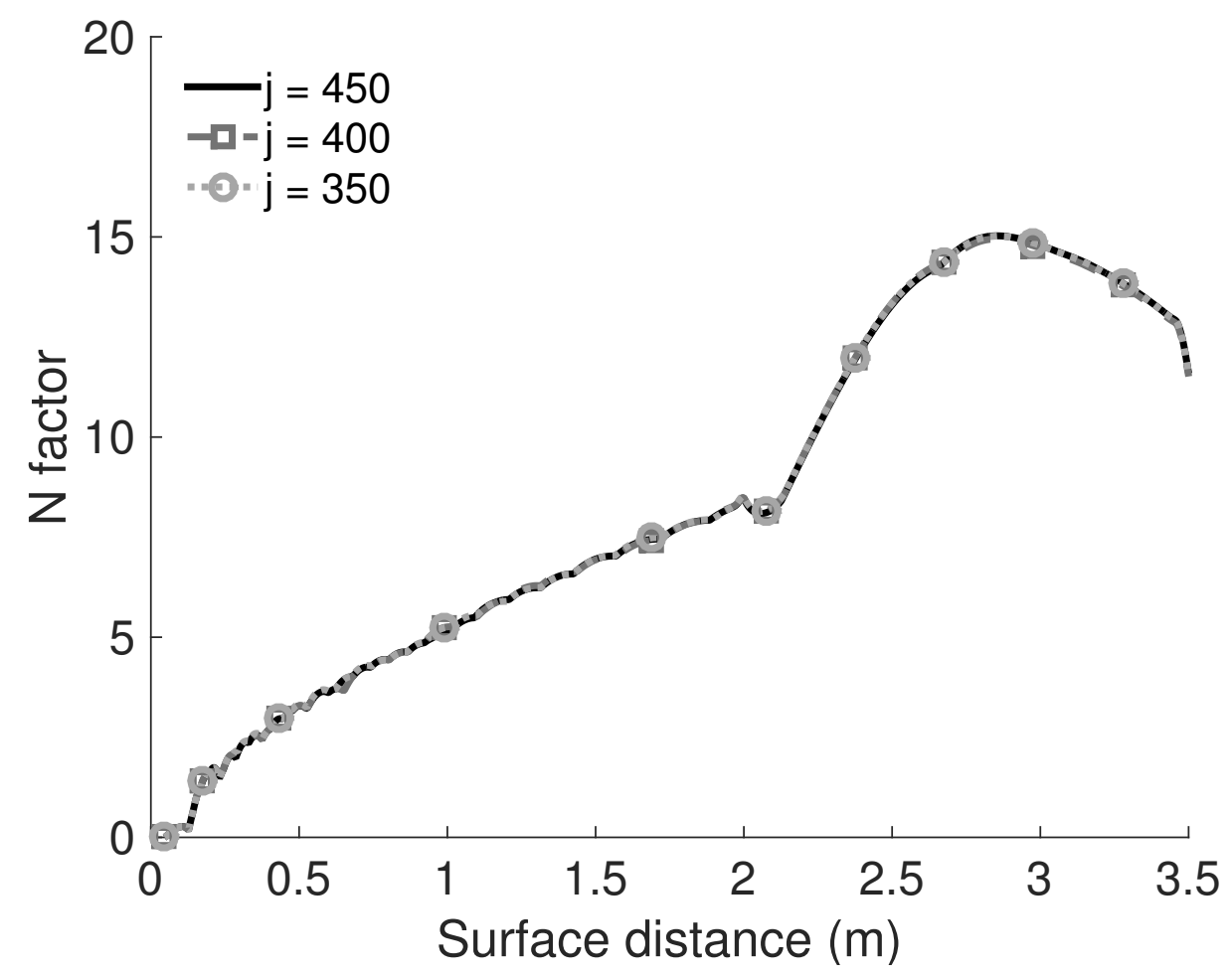

(a) Varying wall normal mean flow grid points.

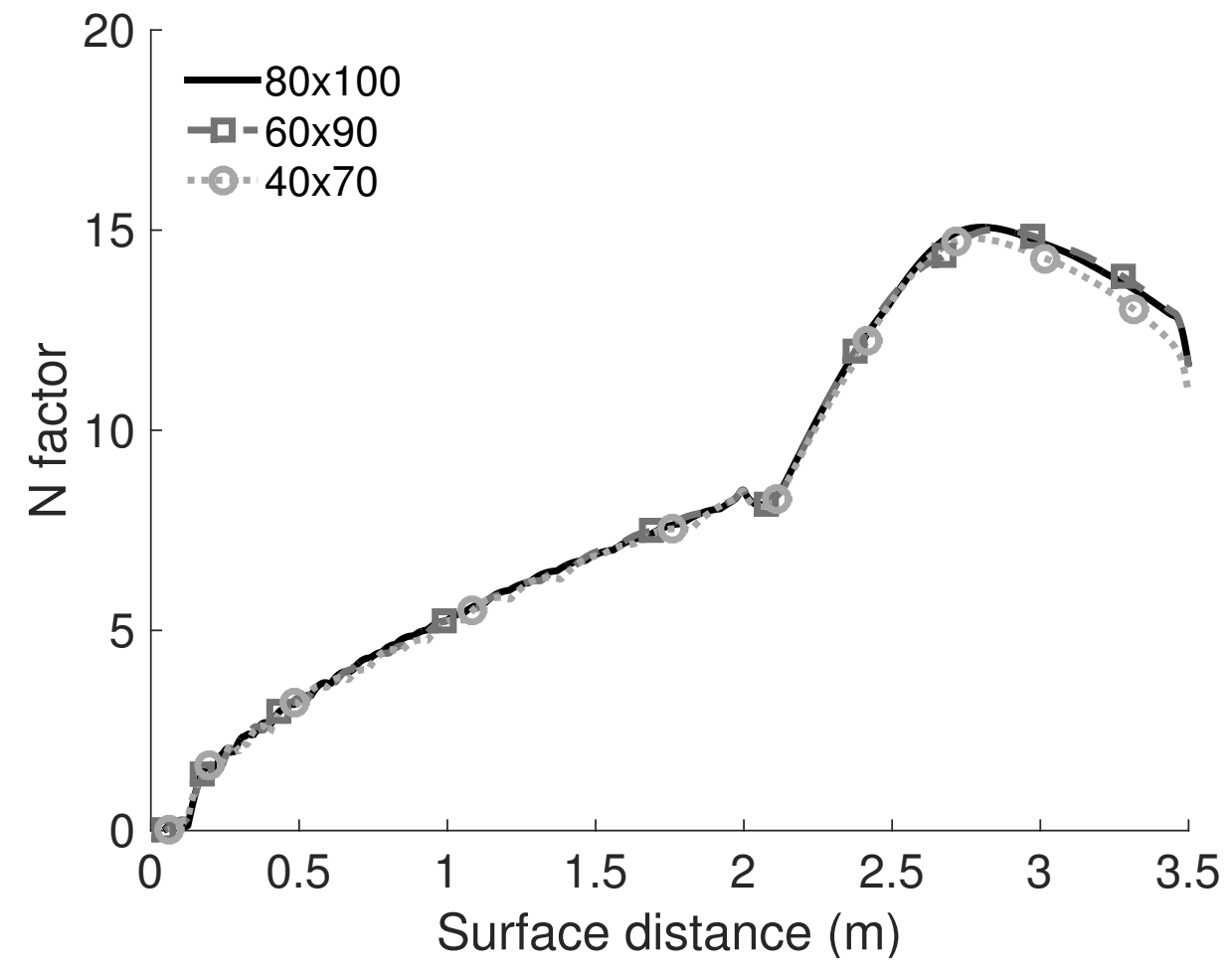

(b) Varying LPSE test matrix (distance $\times$ frequency) density.

Figure 5.3: LPSE resolution study $\left(T_{w}=300 \mathrm{~K}, a_{1-3} / h=4.204,-0.374,0.085\right)$. 
the minimum and maximum bound for each input parameter. The range for each input parameter is listed in Table 5.4, where the modal amplitudes are nondimensionalized by the panel thickness.

Table 5.4: Bounds for surrogate input parameters.

\begin{tabular}{lcc}
\hline \hline Parameter & Minimum & Maximum \\
\hline Temperature, $\mathrm{K}$ & 280 & 420 \\
$1^{\text {st }}$ mode amplitude & -0.838 & 5.037 \\
$2^{\text {nd }}$ mode amplitude & -0.461 & 0.076 \\
$3^{\text {rd }}$ mode amplitude & -0.017 & 0.102 \\
\hline \hline
\end{tabular}

An initial set of 1000 sample points are generated to construct surrogates and assess their accuracy. Surrogates are generated with 100 to 800 sample points, where the number of sample points used for training $\left(n_{s p}\right)$ increases in increments of 100. For a given value of $n_{s p}$, several surrogates are generated using different subsets of the 1000 sample points. The number of surrogates $(d)$ generated with a constant value of $n_{s p}$ is determined according to Eq. (5.1).

$$
d=\frac{1000-n_{s p}}{100}+1
$$

Each of the $d$ surrogates are evaluated against the $1000-n_{s p}$ remaining sample points. This approach, termed cross-validation [126], is used in this study to provide robust error metrics for surrogate convergence with increasing $n_{s p}$, and for a fixed $n_{s p}$, identify which subset of the 1000 sample points results in the most accurate surrogate. An example of the cross-validation technique is provided in 
Table 5.5 for surrogates constructed with 800 sample points. The data in Table 5.5 indicates that $n_{s p}=800$ results in three surrogates that are trained and evaluated against different subsets of the 1000 sample points.

Table 5.5: Cross-validation approach for $n_{s p}=800$ surrogates.

\begin{tabular}{ccc}
\hline \hline Surrogate Number & Training point index & Evaluation point index \\
\hline 1 & $1-800$ & $801-1000$ \\
2 & $101-900$ & $1-100,901-1000$ \\
3 & $201-1000$ & $1-200$ \\
\hline \hline
\end{tabular}

Use of the transition onset surrogate in the aerothermoelastic model introduces two sources of error in the response prediction; model reduction error and surrogate input error. Model reduction error is a result of replacing the full-order CFD/LPSE analysis with the surrogate function. This error is computed by comparing the output of the surrogate and STABL for the same input within the parameter space. Surrogate input error results from assumptions made to parameterize the input variables. Here, this involves neglecting the impact of surface temperature gradients and only using the displacement computed from the first 3 modal weights.

One metric of the model reduction error, the percent error in the $N$ factor curve $\left(N_{E R R O R}\right)$, is shown in Fig. 5.4 for surrogates generated with 100 to 800 training points. The $N$ factor error is computed using Eqs. (5.2)-(5.3), where $N_{M O D E L}$ is the surrogate predicted $N$ factor curve, $N_{S T A B L}$ is the STABL computed $N$ factor curve, $m$ is the number of data points in a single evaluation case, and $i, j$ are indices 
corresponding to the $i$ th data point in the $j$ th evaluation case.

$$
\begin{gathered}
N_{\text {ERROR }}(S)_{j}^{(i)}=\frac{\left|N_{\text {MODEL }}(S)_{j}^{(i)}-N_{S T A B L}(S)_{j}^{(i)}\right|}{R M S_{j}} \times 100 \\
R M S_{j}=\sqrt{\frac{1}{m} \sum_{i=1}^{m}\left(N_{S T A B L}(S)_{j}^{(i)}\right)^{2}}
\end{gathered}
$$

The solid curves in Fig. 5.4 indicate the average $N_{E R R O R}$ across the $d$ surrogates at any value of $n_{s p}$. Examination of Fig. 5.4.a indicates that, by 500 training points, the average $N_{E R R O R}$ is converging to a minimum error near $0.63 \%$. The maximum $N_{E R R O R}$ in Fig. 5.4.b decreases to a minimum value of $12.0 \%$ at $n_{s p}=500$, at which point the error begins to rise. The $n_{s p}=700$ surrogate with the minimum average $N_{E R R O R}$ is chosen for this study as increasing the number of training points to 800 results in greater maximum error and a marginal reduction in the average error.

The model reduction error associated with the $n_{s p}=700$ surrogate is shown in Fig. 5.5 in terms of the $N_{E R R O R}$ and the predicted onset location error $S_{E R R O R}$, which is computed using Eq. (5.4).

$$
S_{E R R O R}(N)_{j}=\frac{\left|S_{M O D E L}(N)_{j}-S_{S T A B L}(N)_{j}\right|}{L} \times 100
$$

A contour of the $N$ factor error is provided in Fig. 5.5.a for each of the 300 evaluation cases. The low error for surface distances less than $2 \mathrm{~m}$ indicates that the surrogate provides an accurate prediction of the $N$ factor curve for variation in the 


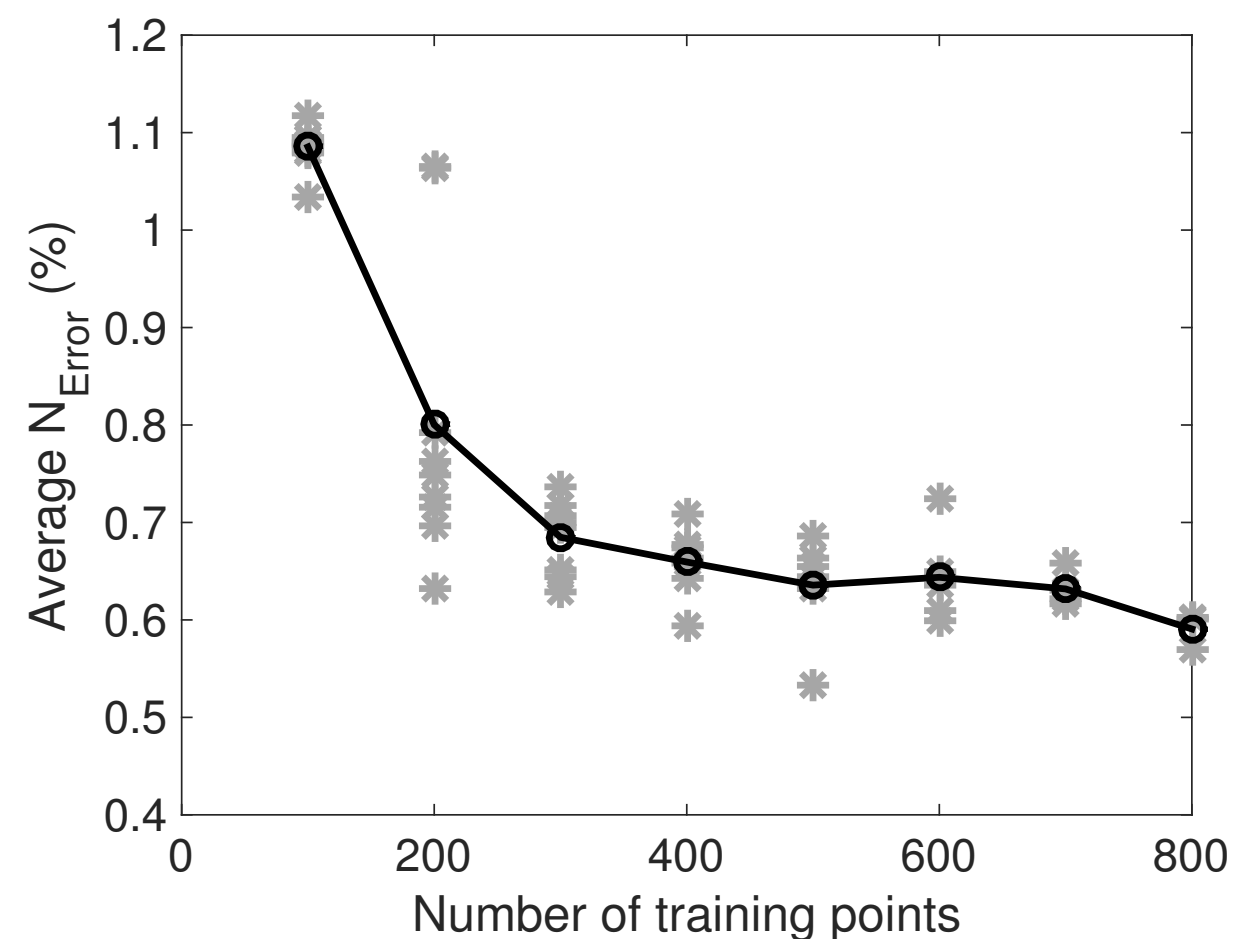

(a) Average $N_{E R R O R}$.

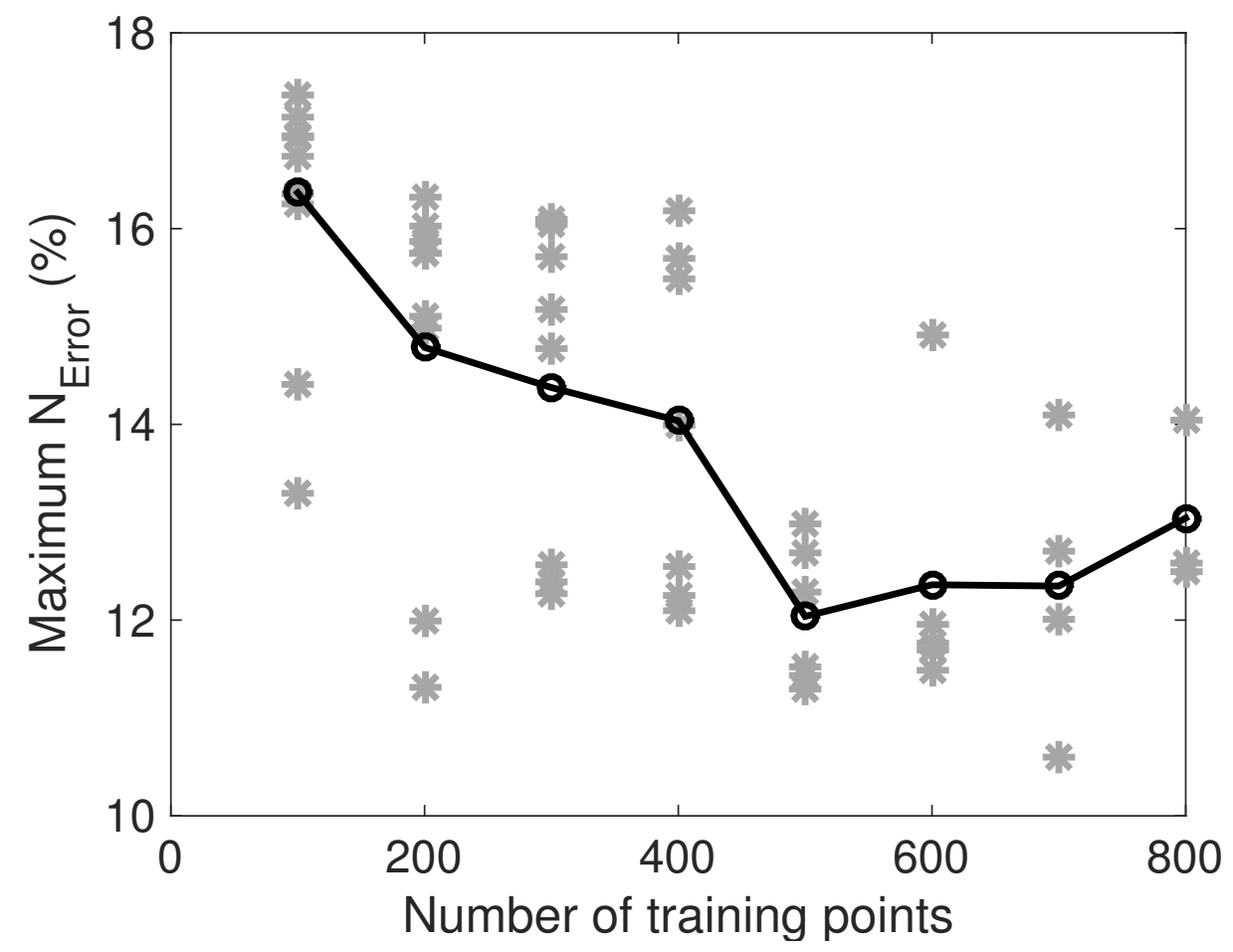

(b) Maximum $N_{E R R O R}$.

Figure 5.4: Surrogate convergence with increasing sample points. 
isothermal wall temperature. The largest error occurs over the panel location (2.0 to $3.5 \mathrm{~m}$ ) and therefore, is a result of the deformation. For the 300 test cases, the maximum $N_{E R R O R}$ is $11.8 \%$ while the mean error is $0.6 \%$. Accuracy of the surrogates transition prediction capability is assessed assuming onset occurs at $N=10$. The surrogate and STABL predicted onset locations, and corresponding error, are shown in Fig. 5.5.b for each of the 300 test cases. The maximum $S_{E R R O R}$ at $N=10$ is $10.3 \% \mathrm{~L}$ while the mean error is $1.6 \% \mathrm{~L}$.

The surrogate input error results from truncating the deformation to the first 3 free vibration modes and neglecting spatial variation in the surface temperature across the panel. As previously stated, comparison of the displacement generated using 3 and 25 mode shapes results in a maximum error of $0.6 h$. In regard to neglecting the thermal gradient, recent work has found excellent agreement between experimentally measured transition onset and STABL predictions, assuming an isothermal wall condition specified as the average temperature in the laminar region [164]. However, in this previous work the variation in wall temperature throughout the transition process $(294$ to $320 \mathrm{~K}$ ) is much smaller than that observed for configurations similar to that studied here [30]. The accuracy of transition prediction based on the laminar region temperature is assessed by evaluating the boundary-layer stability of the flow past the panel response in Fig. 5.6. Maximum $N$ factor curves are obtained using the isothermal surrogate (evaluated at the laminar region temperature) and STABL which accounts for the temperature gradient across the panel. For the STABL predictions, the wall temperature be- 


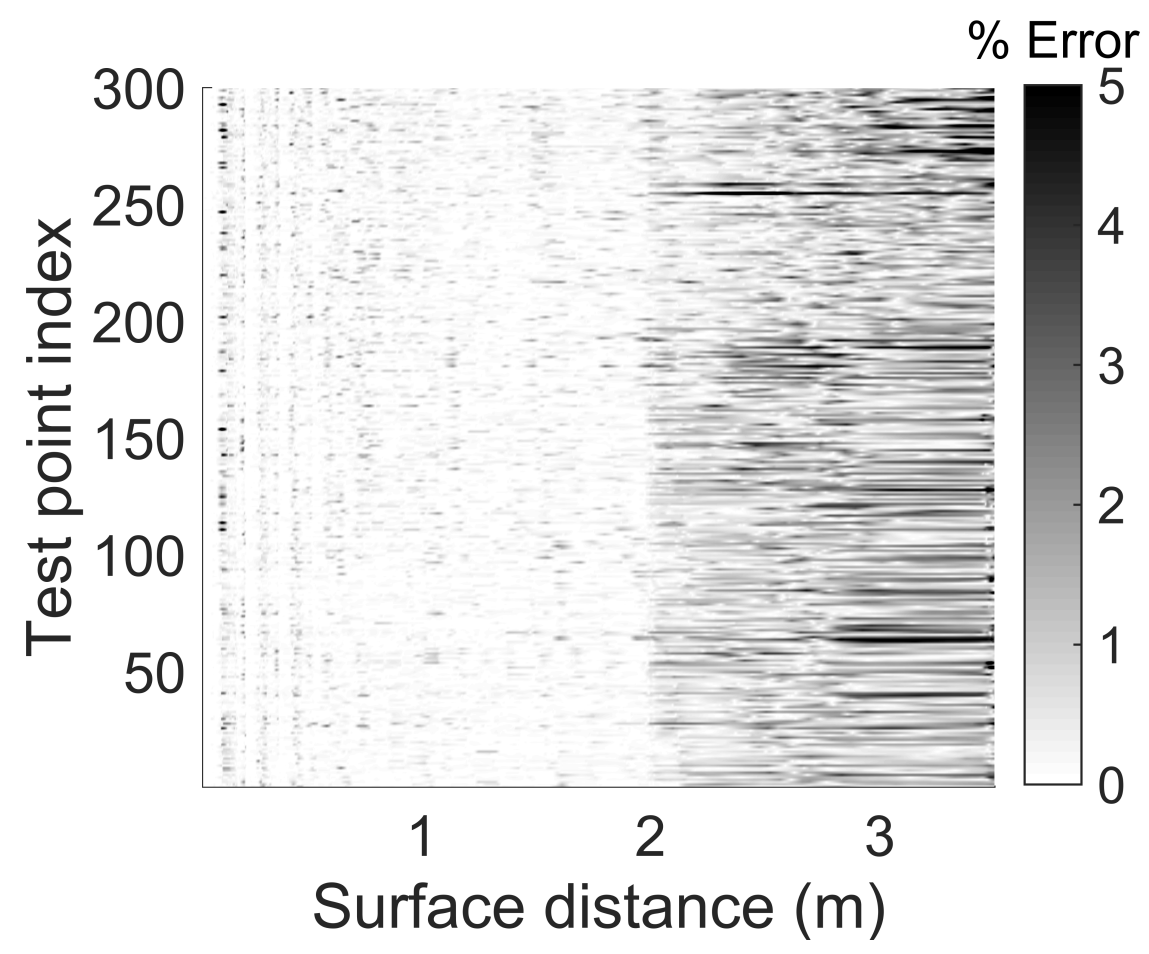

(a) $N$ factor error contour.

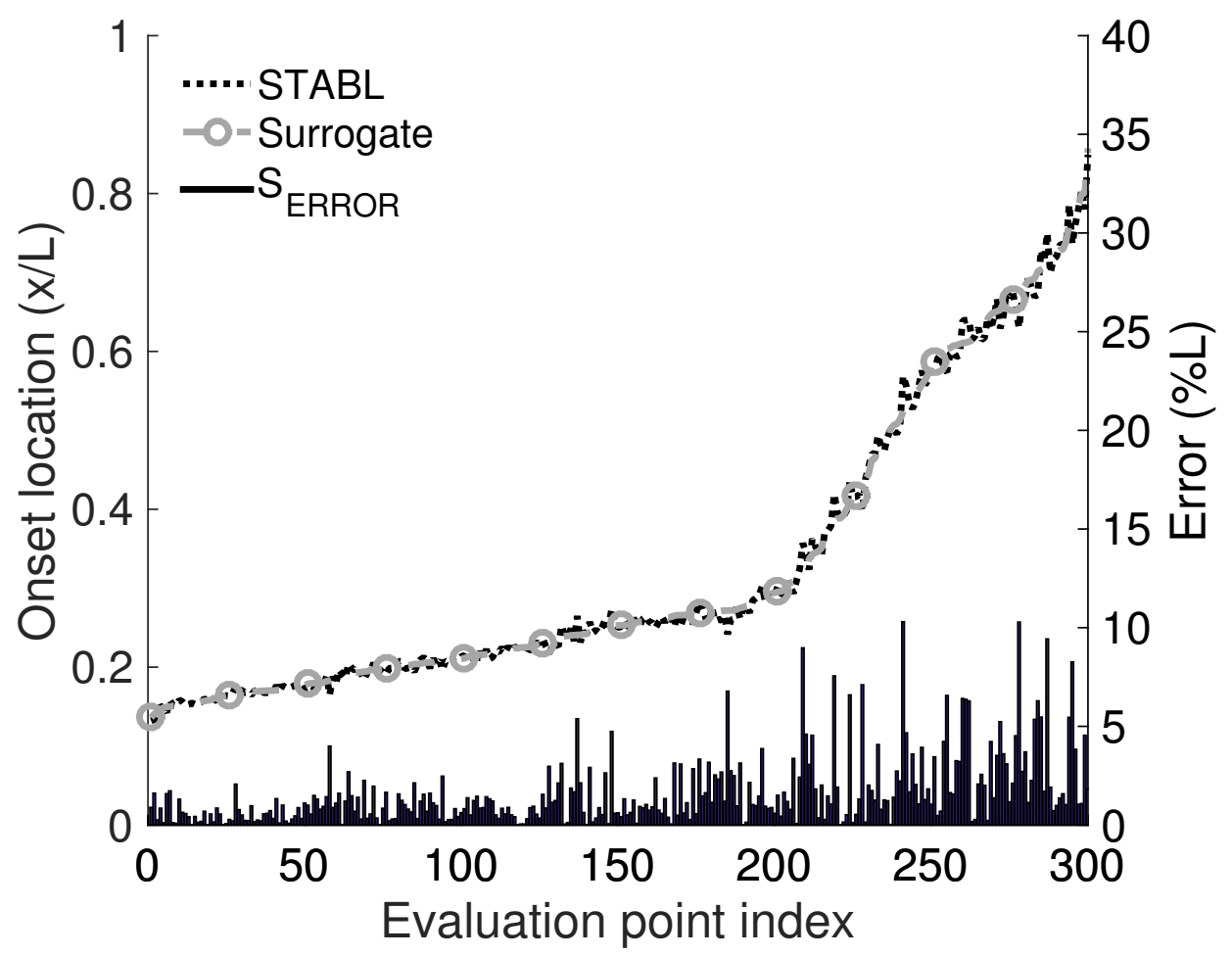

(b) Predicted onset location and error for $N=10$.

Figure 5.5: Model reduction error. 
fore and after the panel is specified to match the temperature at the panel boundaries. STABL predictions for the varying wall temperature are compared with the isothermal surrogate evaluations in Fig. 5.7 for geometries that neglect (Fig. 5.7.a) or account for (Fig. 5.7.b) panel deformation. The markers in Fig. 5.7 indicate the predicted transition onset location assuming $N=10$. A comparison of the $N$ factor curves in Fig. 5.7.a indicates that, for a smooth wall, neglecting the spatial variation in wall temperature can alter the predicted onset location by $0.61 \mathrm{~m}(41 \% L)$. However, when deformation is accounted for (Fig. 5.7.b) the agreement in $N$ factor between STABL and the surrogate extends to approximately $2.4 \mathrm{~m}$, resulting in an accurate surrogate prediction of the onset location. The improved agreement between STABL and the surrogate in Fig. 5.7.b suggests that as the panel deforms, the effect of the temperature gradient on the overall $N$ factor growth becomes less pronounced.

As spatial variation in the wall temperature may be important to onset prediction at small deformations, a temperature correction is applied to the surrogate to partially account for thermal gradients. For a given panel response, the surrogate is evaluated at the minimum (i.e., laminar) and average wall temperature. As the temperature rise is largely due to transition, an intermittency based blending of the minimum and average temperature $N$ factor curves is used to predict the onset location. The improved agreement in $N$ factor resulting from this temperature blending is shown by the curve labeled "Surrogate: $T_{\text {blend }}$ " in Fig. 5.8, where onset occurs at $2.24 \mathrm{~m}$ and the transition length is $0.30 \mathrm{~m}$. 


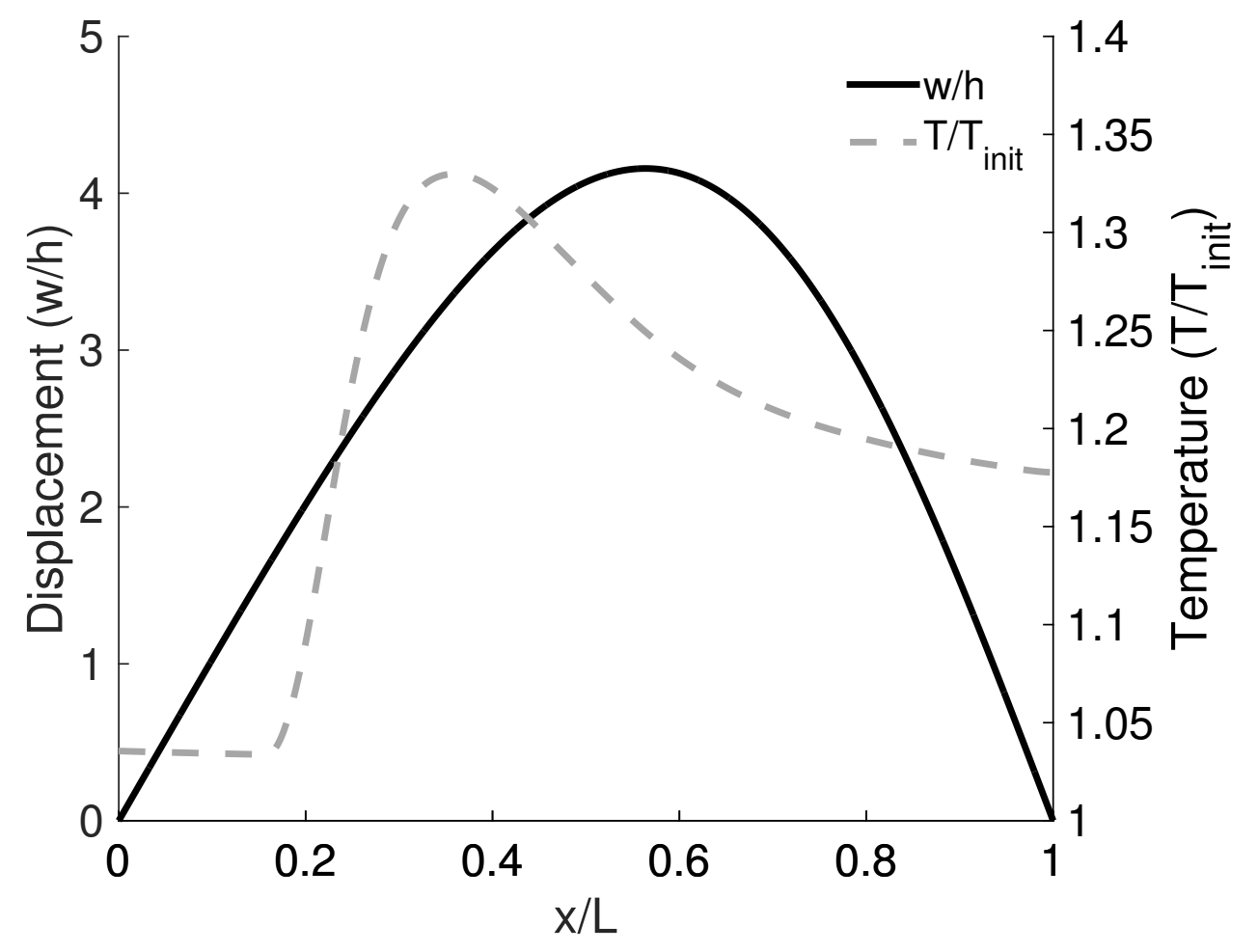

Figure 5.6: Panel response at $10 \mathrm{~s}$ due to time-varying transition region $\left(\Delta x_{t}=0.30\right.$ $\mathrm{m})$. 


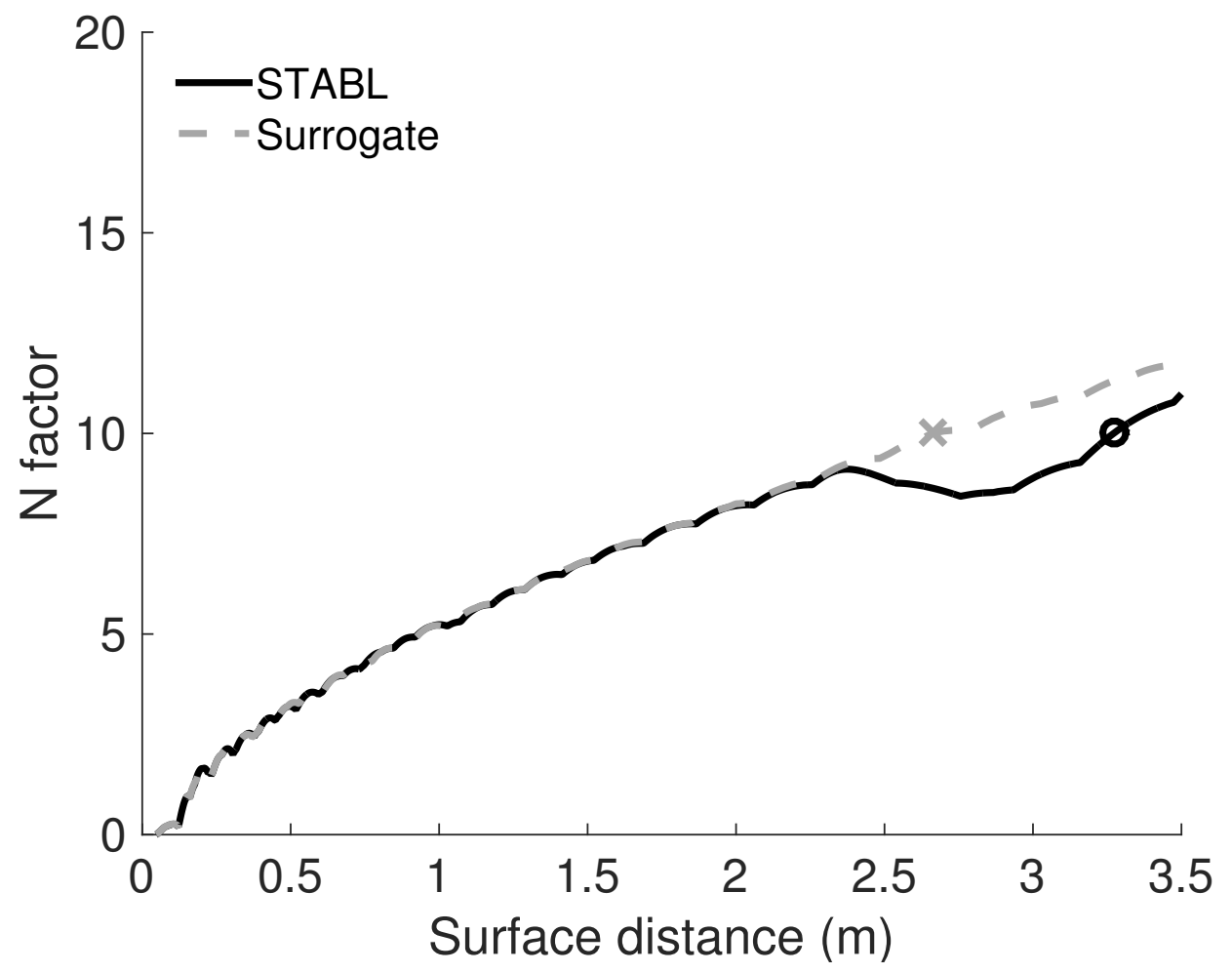

(a) No deformation $\left(a_{1-3} / h=0\right)$.

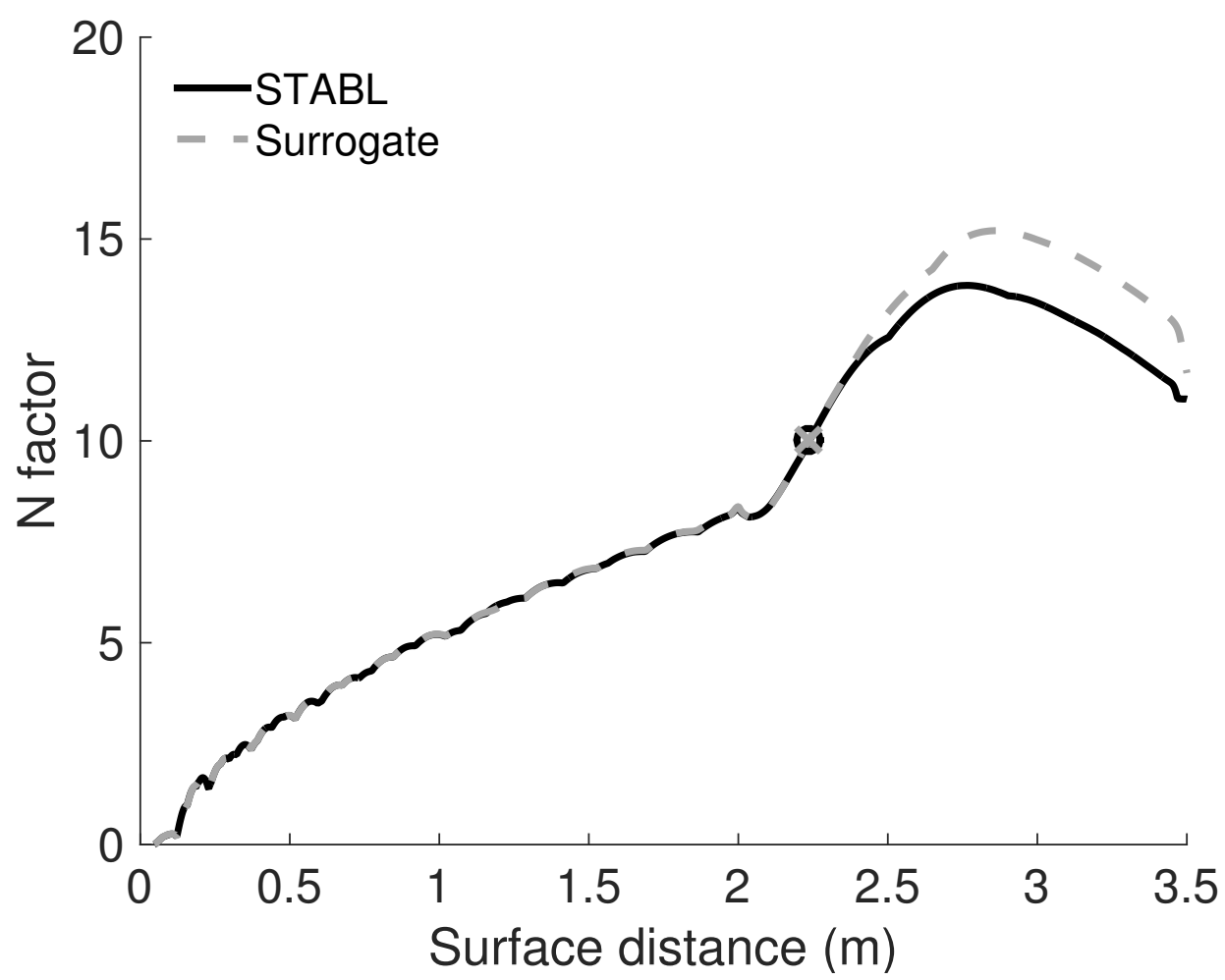

(b) Deformation $\left(a_{1-3} / h=4.0885,-0.4244,0.0175\right)$.

Figure 5.7: Impact of wall temperature gradient on transition prediction. 


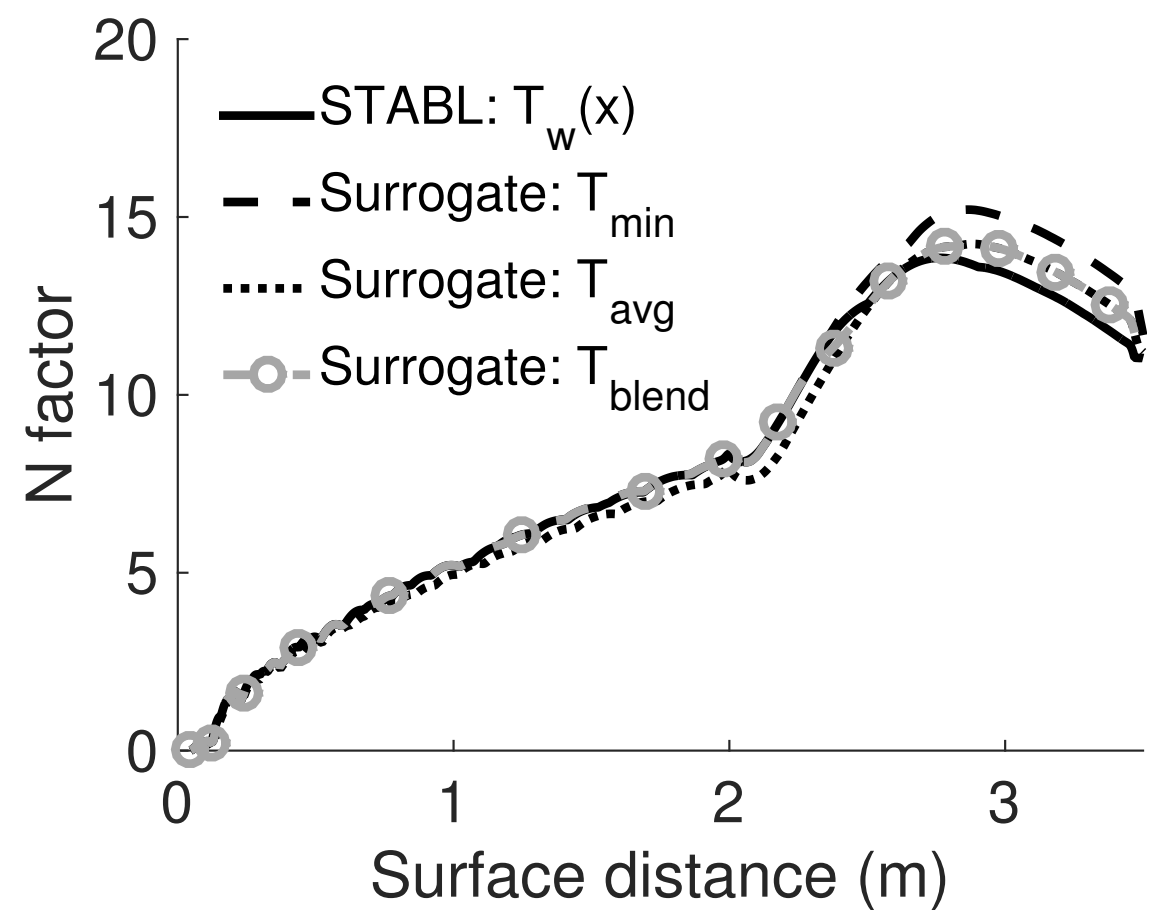

Figure 5.8: Intermittency-based blending of minimum and average temperature $N$ factor curves.

\subsubsection{Surrogate Verification}

The applicability of the surrogate for aerothermoelastic response prediction is assessed by comparing $5 \mathrm{~s}$ of panel response where transition onset is predicted using both the surrogate model and STABL. For either response, the transition onset location is updated every $0.10 \mathrm{~s}$. At this time step, the movement of the onset location is converged within $0.47 \% \mathrm{~L}$. The accuracy of the surrogate model, relative to using STABL for transition prediction, is shown in Fig. 5.9 in terms of the movement of the onset location (Fig. 5.9.a) and associated error metrics (Fig. 5.9.b). Note that the percent errors in Fig. 5.9.b for the onset location, mid-chord displacement, and maximum temperature are normalized by the length $L$, thickness $h$, and ini- 
tial temperature $T_{\text {init }}$ of the panel. A comparison of the onset locations in Fig. 5.9.a reveals that, in general, the surrogate predicts transition further upstream than STABL. Note that the initial surrogate prediction for the undeformed panel at $t=$ $0 \mathrm{~s}$ is $0.054 \mathrm{~m}(3.6 \% L)$ further upstream than the STABL predicted onset location. The error in onset location further into the response could result from this initial discrepancy. As shown in Fig. 5.9.b, the error in the onset location results in maximum displacement and temperature errors of $2.6 \% \mathrm{~h}(0.13 \mathrm{~mm})$ and $0.3 \% T_{\text {init }}(0.74$ K). The computational cost associated with generating $5 \mathrm{~s}$ of response using STABL and the surrogate model is provided in Table 5.6. The comparison in Table 5.6, along with the relatively low error evident in Fig. 5.9.b, highlights the strength of the surrogate approach in replacing the need to perform STABL computations in the aerothermoelastic solution process. The wall and CPU times for the surrogate in Table 5.6 do not include the time required to generate the training points and construct the surrogate. If the time to generate the training data is accounted for the surrogate is approximately 10 times the computational expense of the STABL prediction. However, the surrogate can be used to quickly perform parametric sweeps of the initial transition region (onset and length), wall temperature, and panel deformation. 


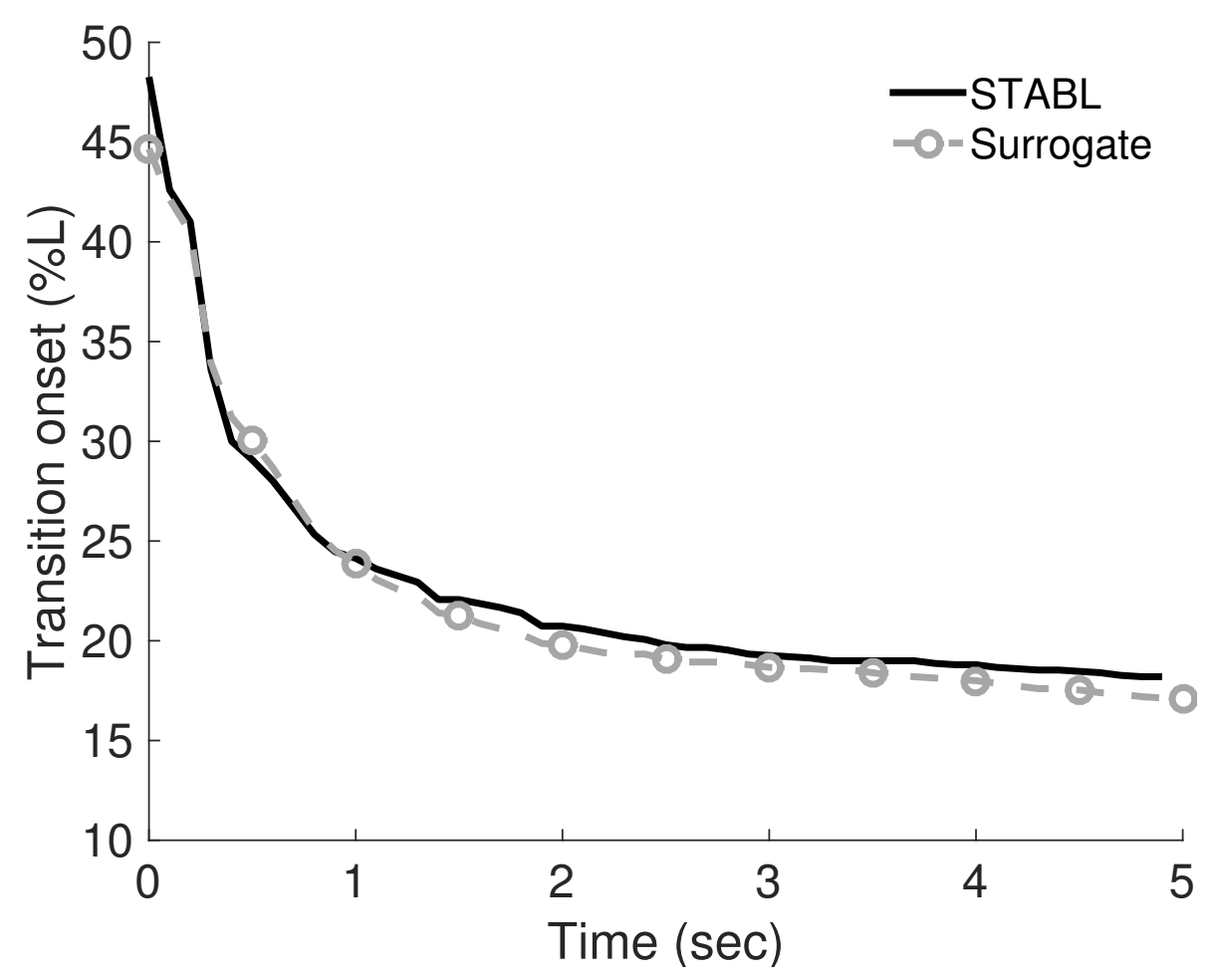

(a) Onset location.

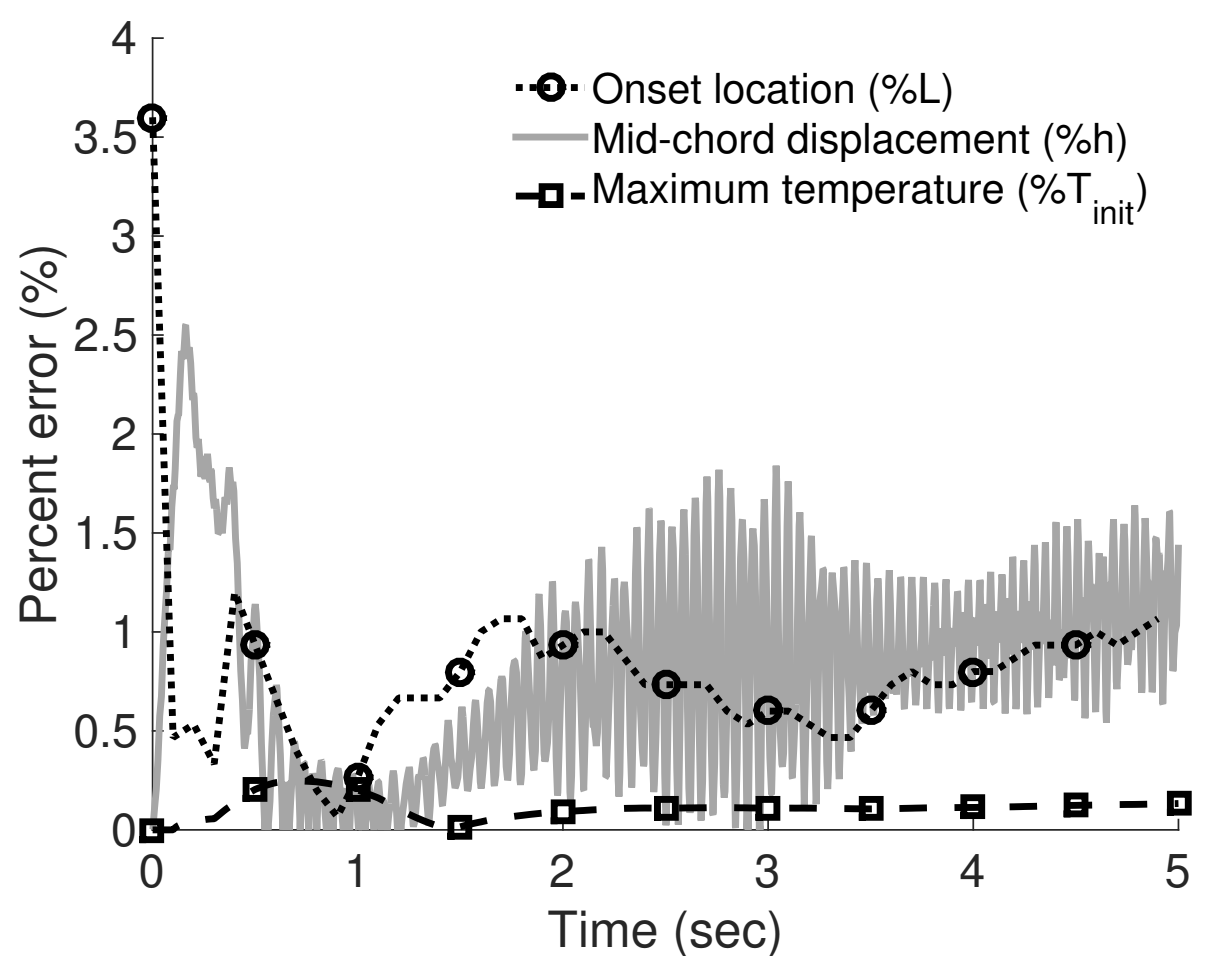

(b) Percent errors.

Figure 5.9: Comparison of the aerothermoelastic panel responses obtained using STABL and the surrogate for transition prediction. 
Table 5.6: Computational cost for $5 \mathrm{~s}$ of aerothermoelastic panel response.

\begin{tabular}{lccc}
\hline \hline Transition prediction & Processor cores & Wall time, hrs & CPU time, hrs \\
\hline STABL & 144 & 67.7 & 9748.8 \\
Surrogate $^{\mathrm{a}}$ & 1 & 1.6 & 1.6 \\
\hline \hline
\end{tabular}

${ }^{\text {a }}$ Each surrogate sample point required $\approx 2.72 \mathrm{hrs}$ on 36 processor cores. Generation of the surrogate (1,000 sample points) resulted in total wall and CPU times of 2716.7 and $97801 \mathrm{hrs,} \mathrm{respectively.}$

\subsubsection{Clamped Panel Surrogate Modifications}

The surrogate model described in Sections 5.2.2 and 5.2.3 is modified to allow for transition prediction for flow past a clamped panel. Prior to predicting transition onset during a clamped panel aerothermoelastic simulation, the modal weights are transformed, using Eq. 5.5, into the simply-supported modal weights that best capture the deformation. Where $a$ and $\phi$ in Eq. 5.5 correspond to the free vibration modal weights and mode shapes for the clamped $(C C)$ and simply-supported $(S S)$ panel.

$$
a_{s s}=a_{c c} \phi_{c c} \phi_{s s}^{T}\left[\phi_{s s} \phi_{s s}^{T}\right]^{-1}
$$

This transformation is necessary as the transition onset surrogate's training data and input parameters correspond to simply-supported panel deformations.

Aerothermoelastic simulations of the clamped panel, subject to fully turbulent and fixed transitional loading, indicate that the minimum bound for the third modal amplitude in Table 5.4 is insufficient to capture $10 \mathrm{~s}$ of response. Therefore, an additional 100 sample points are added to the surrogate to expand the 
minimum bound to $a_{3}=-0.9824$. The expanded parameter space increases the maximum and average $N_{\text {error }}$ to $22.25 \%$ and $1.19 \%$. However, for transition onset at $N=10$, the maximum $S_{\text {error }}$ decreases to $9.4 \% L$ while the average remains constant at $1.6 \% L$.

To justify the use of the simply-supported surrogate for clamped panel transition prediction, clamped and approximated simply-supported panel deformations are generated using the modal amplitudes of each of the 1000 sample points used to construct the surrogate. The average displacement error, across the length of the panel, for each sample point is shown in Fig. 5.10.a in terms of the percent panel thickness $(\% h)$. The clamped and simply-supported approximation of the panel displacement is provided in Fig. 5.10.b for sample point 744, which has the largest average error of $18.3 \% h$ and is highlighted in Fig. 5.10.a. Note that, the simplysupported approximation closely matches the peak deformation of the clamped panel, with the highest error occurring near the trailing edge.

The boundary-layer stability of flow over the deformations in Fig. 5.10.b is assessed using STABL, assuming an isothermal wall at $300 \mathrm{~K}$. The maximum $N$ factor curves, corresponding to each deformation, are plotted in Fig. 5.11. The similarity in the $N$ factor curves results in negligible differences in the predicted onset location for a wide range of transitional $N$ factors. For instance, assuming transition occurs between an $N$ factor of 8 and 10, the maximum error in the predicted onset location is $3.7 \% L$. This results in Fig. 5.11, along with the Glass and Hunt dome study in Section 3.3.3, demonstrate that the maximum $N$ factor envelope is largely 


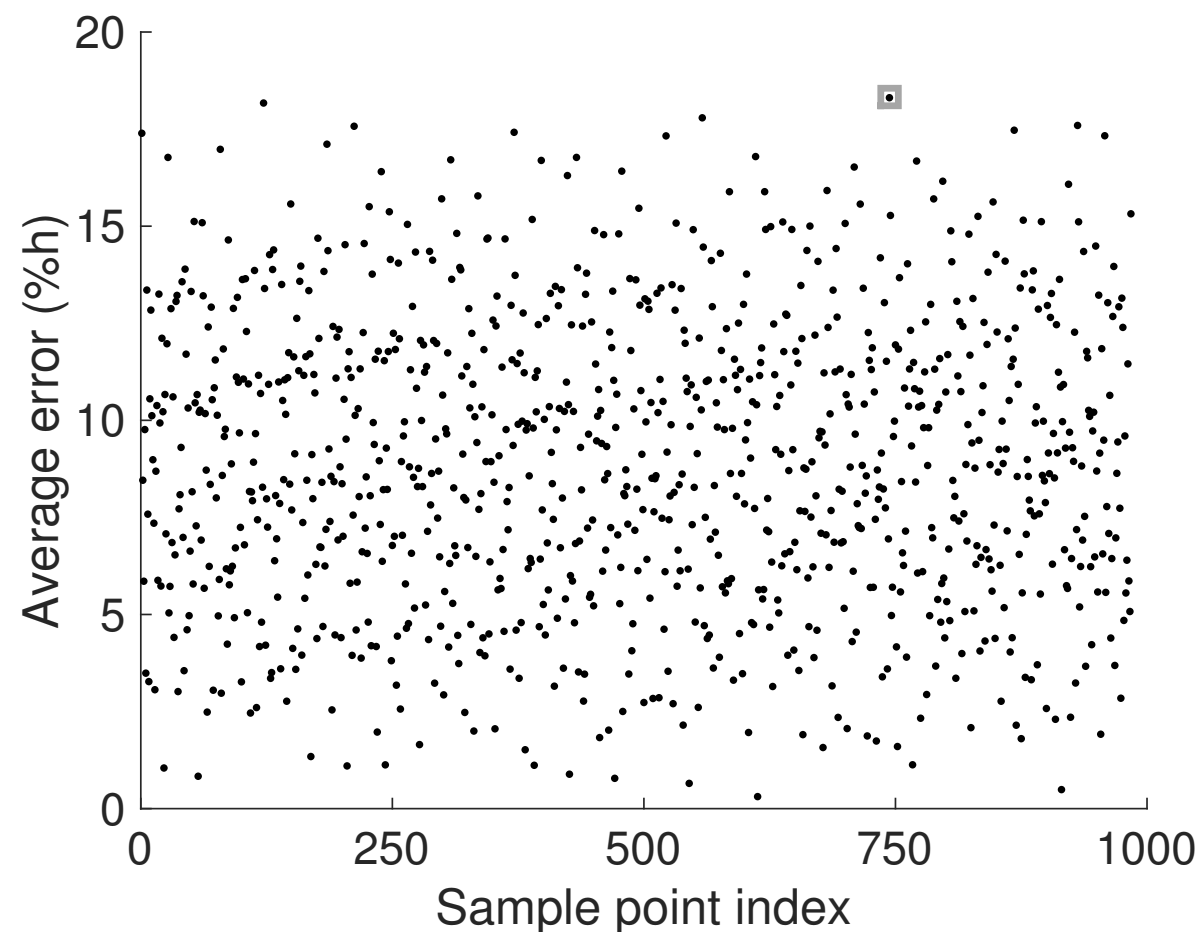

(a) Average displacement error along the length of the panel.

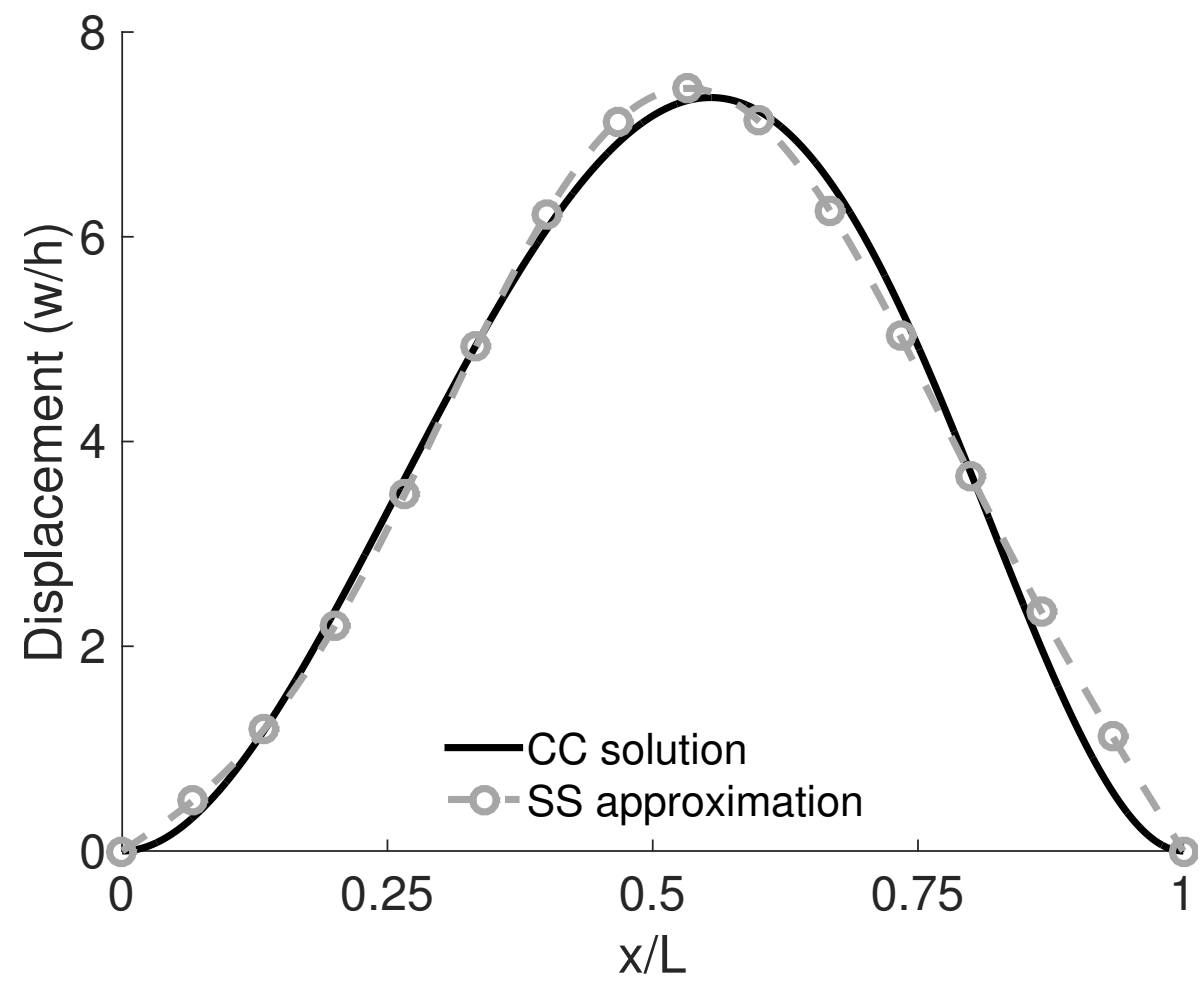

(b) Displacement profiles for sample point 744 .

Figure 5.10: Error associated with approximating clamped panel deformation as simply-supported. 


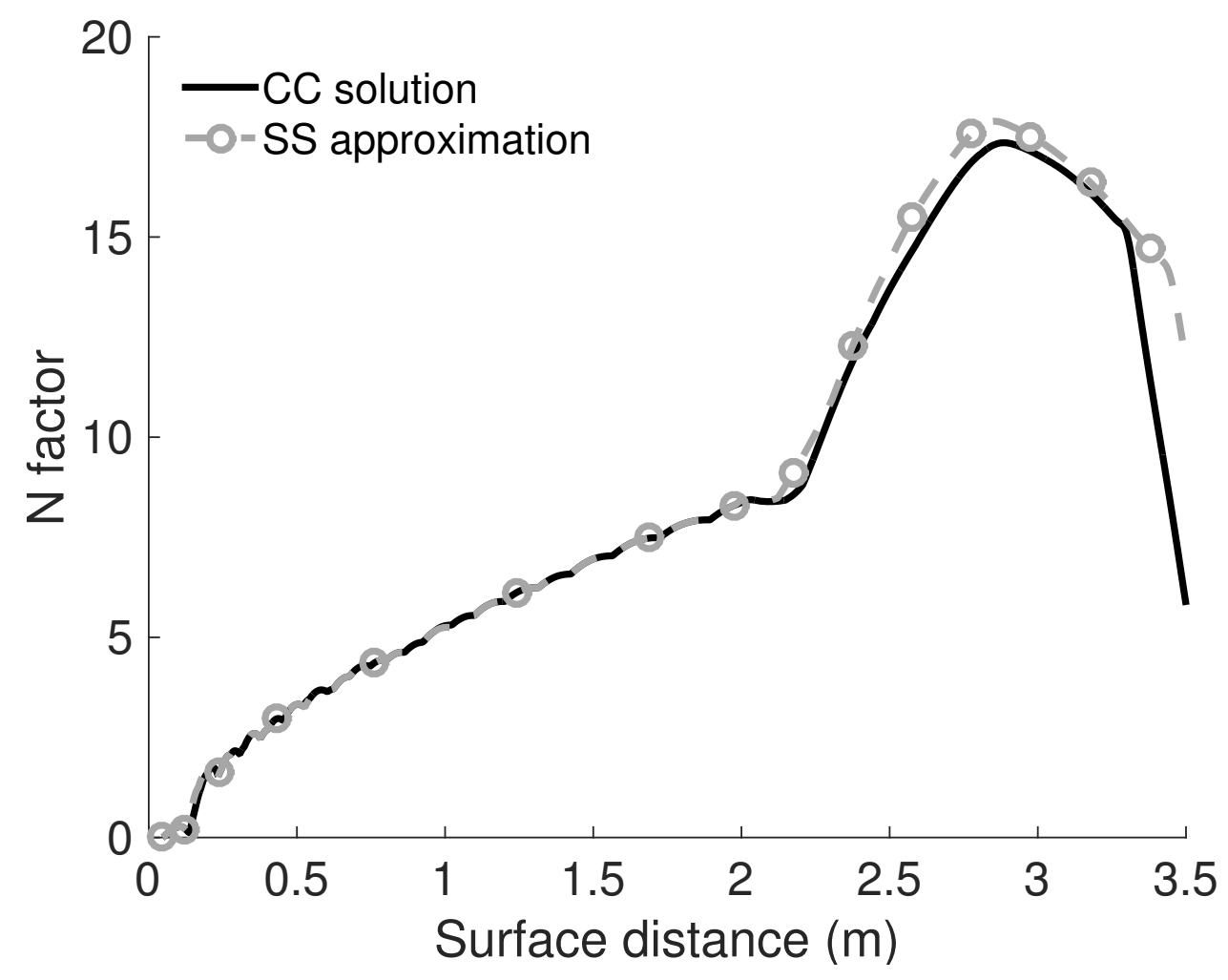

Figure 5.11: Maximum $N$ factor envelope for clamped and simply-supported deformations.

insensitive to the choice of clamped or simply-supported structural boundary conditions.

\subsection{Results}

The importance of accounting for time-varying transition onset in aerothermoelastic response prediction is assessed through a comparison of panel responses obtained assuming fully turbulent and transitional fluid loading. The onset location either remains fixed or varies in time as a function of the panel deformation and wall temperature. As discussed in Section 2.3.1, rearward movement of the 
transition onset location (i.e., relaminarization) is not accounted for. The impact of the panel response on the movement of the transition region is described in Section 5.3.1. Variations in the structural response resulting from a time-varying transition region and changes in the transitional $N$ factor are discussed in Sections 5.3.2 and 5.3.3.

\subsubsection{Effect of Panel Response on Boundary-Layer Transition}

The movement of the transition onset location, at $N=10$, due to the panel response is shown in Fig. 5.12 for both the simply-supported and clamped boundary conditions. For either boundary condition, the onset location moves upstream in time, with the largest movement occurring early in the panel response. Examination of the simply-supported case in Fig. 5.12 indicates that during the first $4 \mathrm{~s}$ of response the onset location shifts upstream by $26.7 \% \mathrm{~L}$. The movement of the onset location is less than $2.3 \% L$ for the remainder of the response. For the clamped panel, the onset location remains fixed at $44.7 \% L$ for the first $0.7 \mathrm{~s}$ of response. This is a result of the clamped panel being less prone to deformation with thermal loading. Once the clamped panel begins to deform, the onset location shifts upstream by $24.2 \% L$ during the first $5 \mathrm{~s}$, with a $2.3 \% L$ variation for the remainder of the response.

\subsubsection{Importance of Panel Deformation and Surface Temperature}

Trends in the predicted onset location for the 1000 sample points generated to construct the surrogate provide insight into the movement of the transition front ob- 


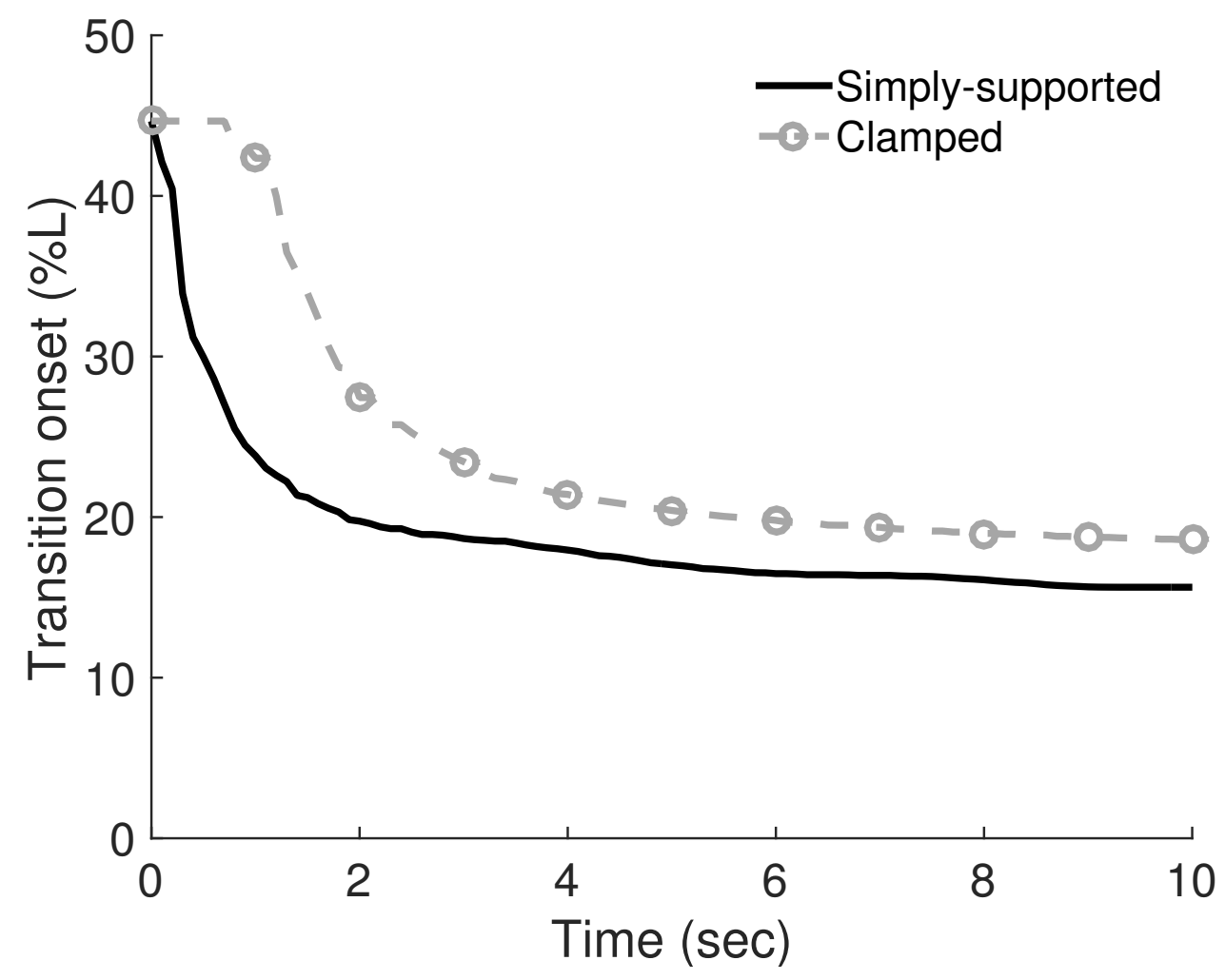

Figure 5.12: Movement of the transition onset location due to the panel response. 
served in Fig. 5.12. For each sample point, the onset location at $N=10$ is plotted against the surface temperature and maximum panel displacement in Fig. 5.13. The data points are contoured with the maximum displacement in Fig. 5.13.a and surface temperature in Fig. 5.13.b. The results in Fig. 5.13.a indicate that, for a constant deformation, increasing the surface temperature moves the transition onset downstream. This finding is consistent with the early work of Mack [42], which demonstrated that surface heating stabilizes second-mode instabilities. At a constant surface temperature in Fig. 5.13.a, increasing the panel deformation moves the onset location upstream. The forward movement of the transition front with displacement is further illustrated in Fig. 5.13.b. The decreased horizontal spread of the predicted onset locations in Fig. 5.13.b demonstrates that, for this panel configuration, deformation plays the dominant role in the movement of transition. Additionally, Fig. 5.13.b reveals that the onset location does not continue to move upstream when the peak displacement exceeds $w / h \approx 2$. For example, "Sample 664 " in Fig. 5.13.b predicts the same onset location $(x / L=0.21)$ as "Sample 956" despite a factor of two difference in the peak displacement. In light of the trends observed in Fig. 5.13, the forward movement of the transition onset in Fig. 5.12 is a result of the panel deformation, which has a more pronounced effect on the onset location than surface temperature. Furthermore, the decreased movement of the onset location later in the response is a result of the panel deformation exceeding $w / h \approx 2$. 


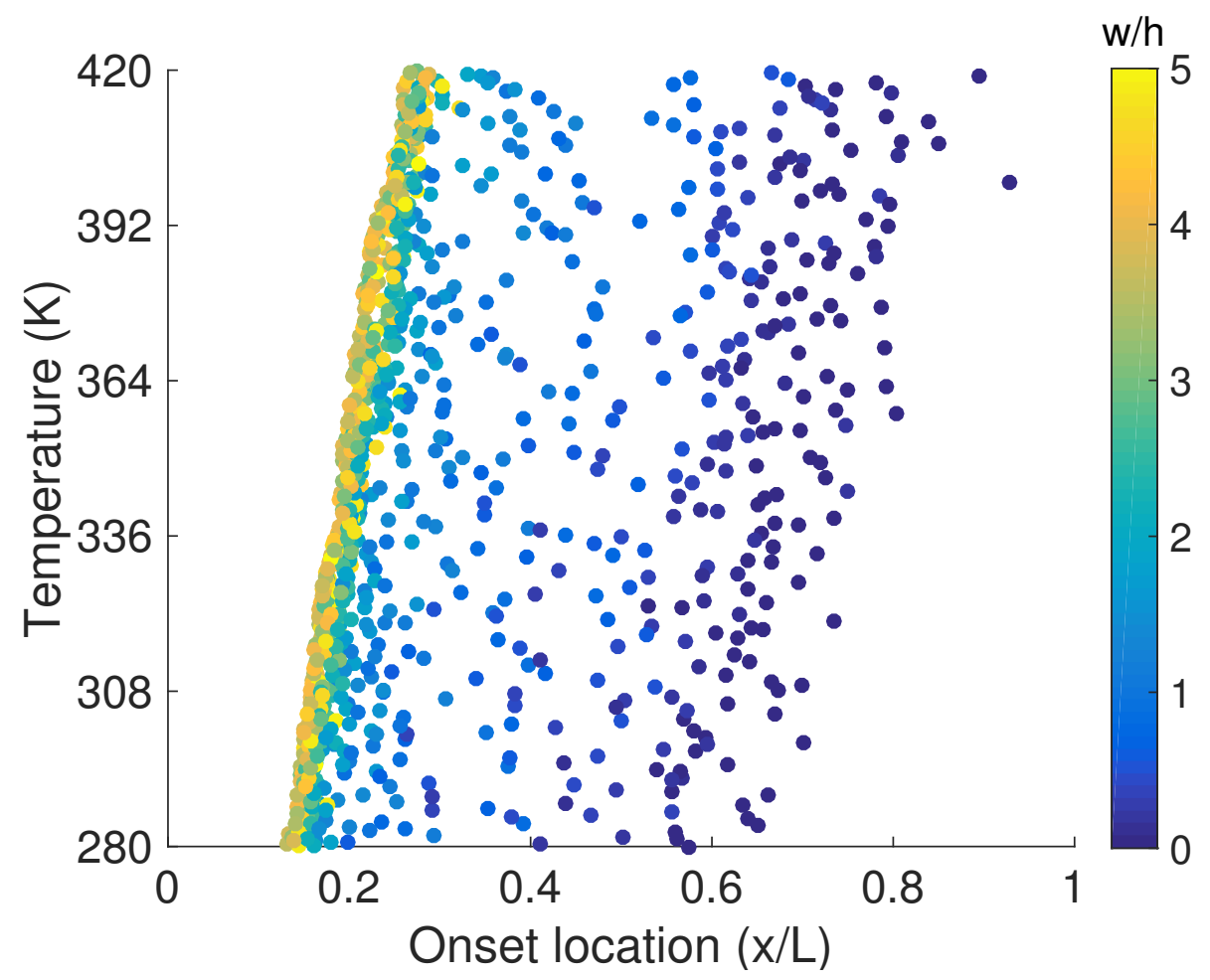

(a) Surface Temperature.

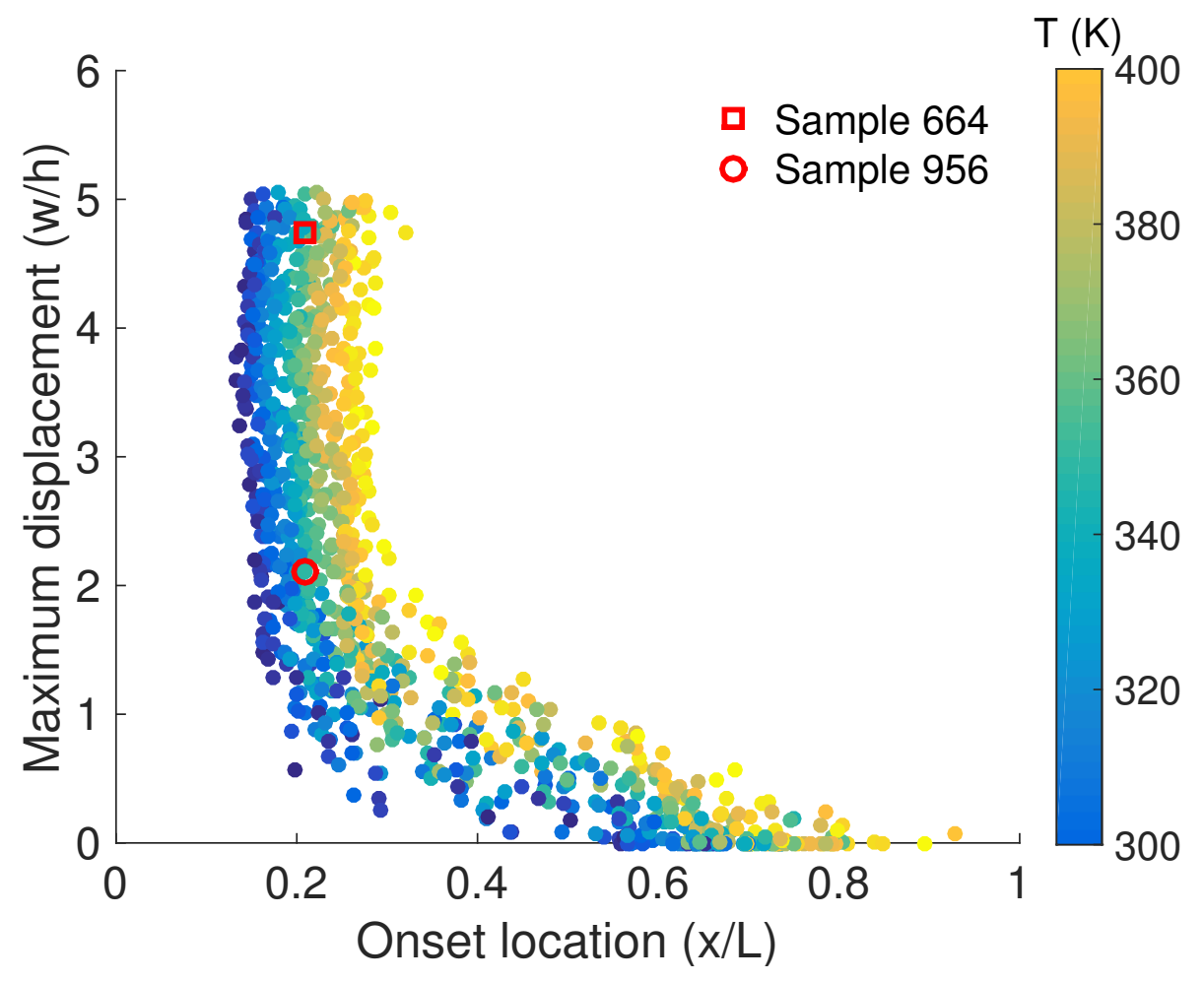

(b) Panel Deformation.

Figure 5.13: Variation in the transition onset location due to the panel response. 


\subsubsection{Physical Explanation for Movement of Transition with Panel Deforma- tion}

The maximum $N$ factor envelopes corresponding to sample points 664 and 956 are provided in Fig. 5.14.a. The agreement in $N$ factor upstream of the panel location indicates the $15 \mathrm{~K}$ difference in wall temperature does not alter the stability of the boundary layer. Therefore, the difference in the Sample 664 and Sample $956 \mathrm{~N}$ factor curves is entirely due to the panel deformation. The results in Fig. 5.14.a show that the maximum $N$ factor and $N$ factor slope over a region of the forward panel (2.1 to $2.7 \mathrm{~m}$ ) increase with deformation. The surface distance (i.e., onset location) corresponding to $N=10$ remains relatively constant despite the increased $N$ factor slope. However, the insensitivity of the onset location to the increased panel displacement is dependent on the assumed value of $N$ at transition. The $N$ factor curves in Fig. 5.14.a clearly indicate the onset location would continue to move upstream if transition occurred at larger $N$ values.

The average slope of the $N$ factor envelope from 2.1 to $2.7 \mathrm{~m}$ is plotted in Fig. 5.14.b for each of the sample points generated to construct the surrogate. The results display a clear trend of increasing $N$ factor slope over the forward panel with increasing displacement. This trend can be explained through examination of the mean flows corresponding to sample points 664 and 956 in Fig. 5.15. For either sample point, the panel deformation results in a region of adverse pressure (Fig. 5.15.a) and suppressed boundary-layer thickness (Fig. 5.15.b) near the leading 
edge of the panel at a surface distance of $2 \mathrm{~m}$. The larger displacement of Sample 664 increases the magnitude and length of the adverse pressure gradient resulting in similar trends in the reduction of the boundary-layer thickness. The frequency of the second-mode instability is inversely proportional to the boundarylayer thickness. Therefore, the reduction in boundary-layer thickness, near the leading edge of the panel, increases the frequency of the second-mode instability. This is confirmed through the stability diagrams in Fig. 5.16, where the frequency increase near $2 \mathrm{~m}$ excites disturbances that are unstable upstream. The continued excitation of these high frequency disturbances, resulting from the region of adverse pressure gradient, increases the maximum value (Fig. 5.17.a) and slope (Fig. 5.17.b) of the $N$ factor envelope. Note that, the ability of adverse pressure gradients to promote earlier transition was experimentally observed by Kimmel [76] for Mach 8 flow past a flared cone in the Arnold Engineering Development Center tunnel B facility. The relatively constant onset location for deformations exceeding $w / h \approx 2$ could be a result of the most unstable frequency continuing to increase with the pressure gradient. In Fig. 5.16 higher frequency disturbances are shown to stabilize prior to the panel location. This decreases their $N$ factor which alters the point where the maximum $N$ factor curve slope increases over the panel as shown in Fig. 5.14a. 


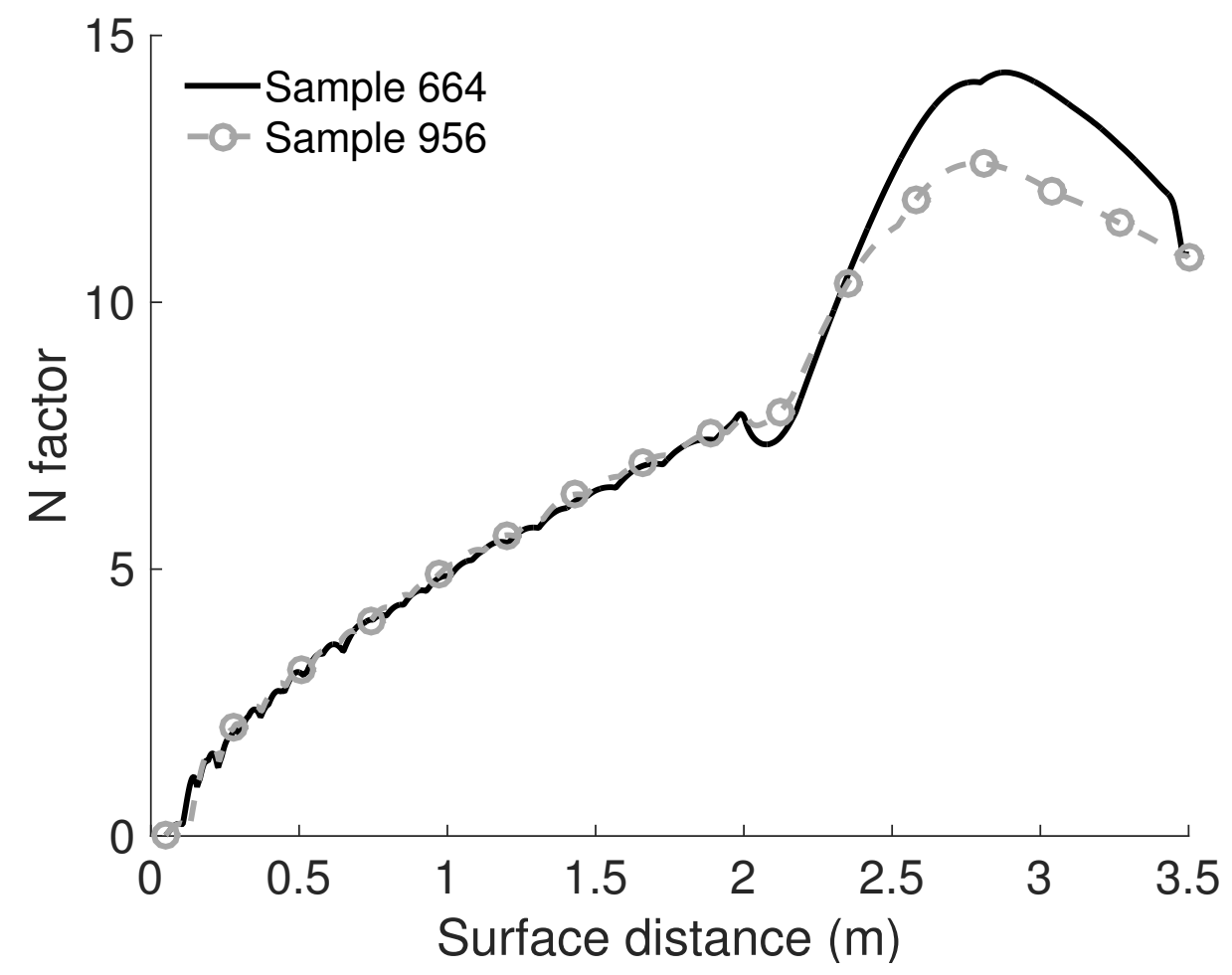

(a) Maximum $N$ factor envelope.

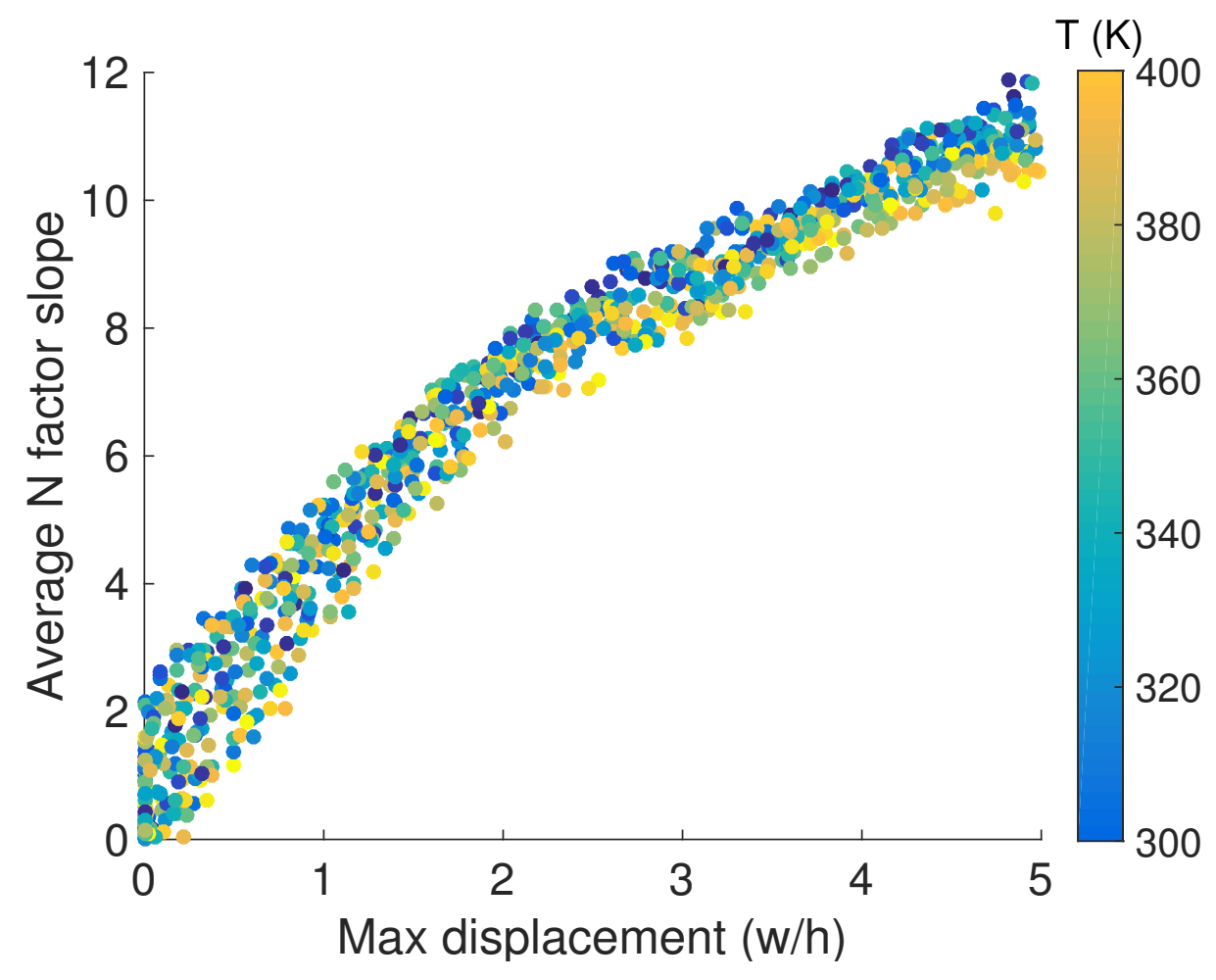

(b) Average $N$ factor slope from 2.1-2.7 m.

Figure 5.14: Effect of panel displacement on $N$ factor growth. 


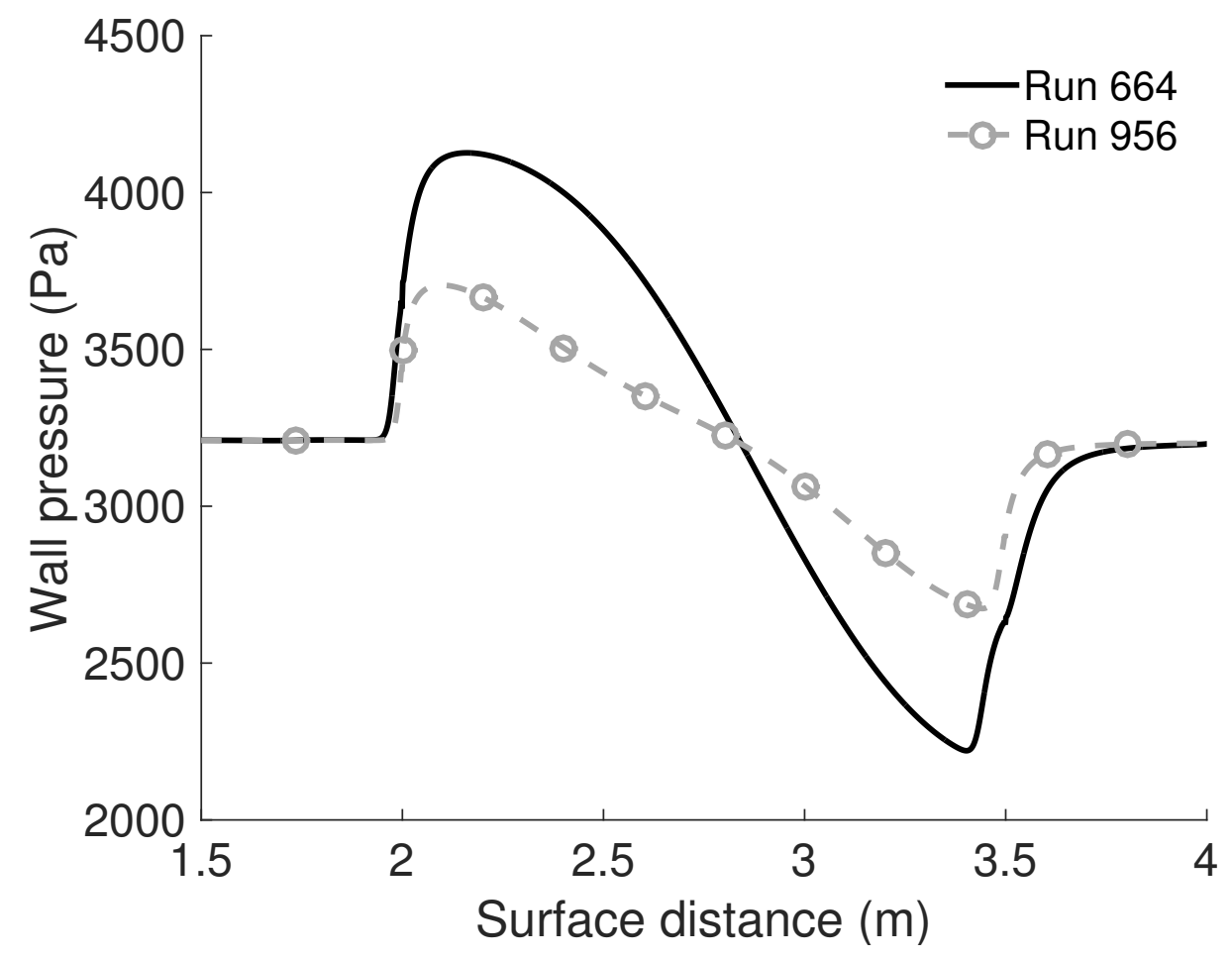

(a) Wall pressure.

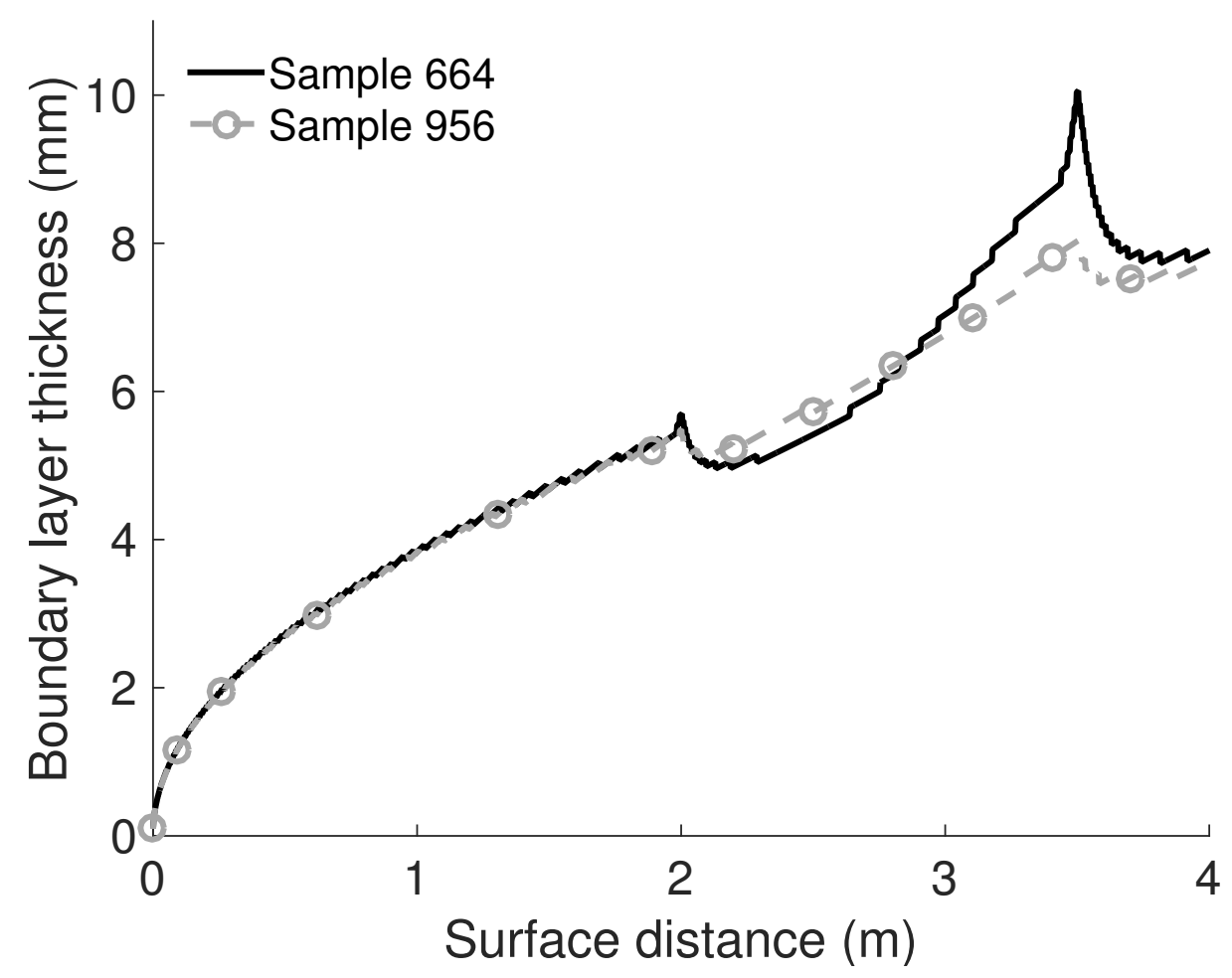

(b) Boundary-layer thickness.

Figure 5.15: Effect of panel deformation on the flowfield. 


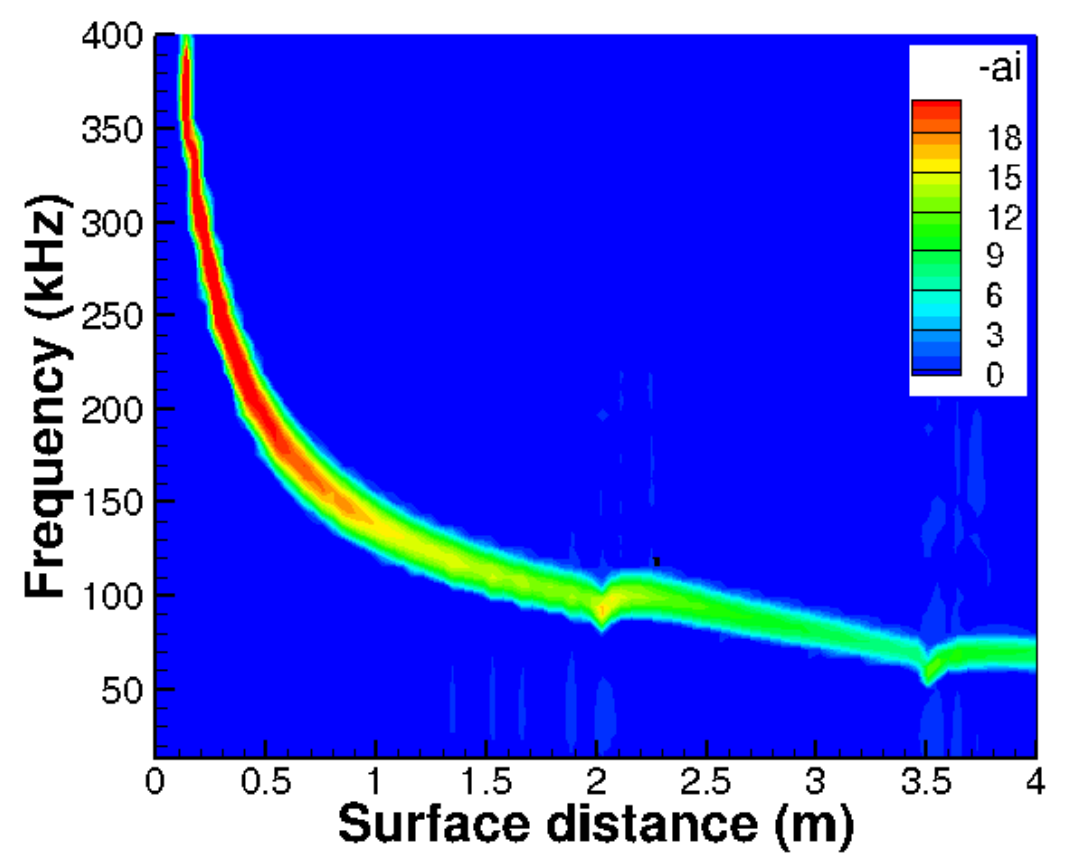

(a) Sample $956(w / h=2.11)$.

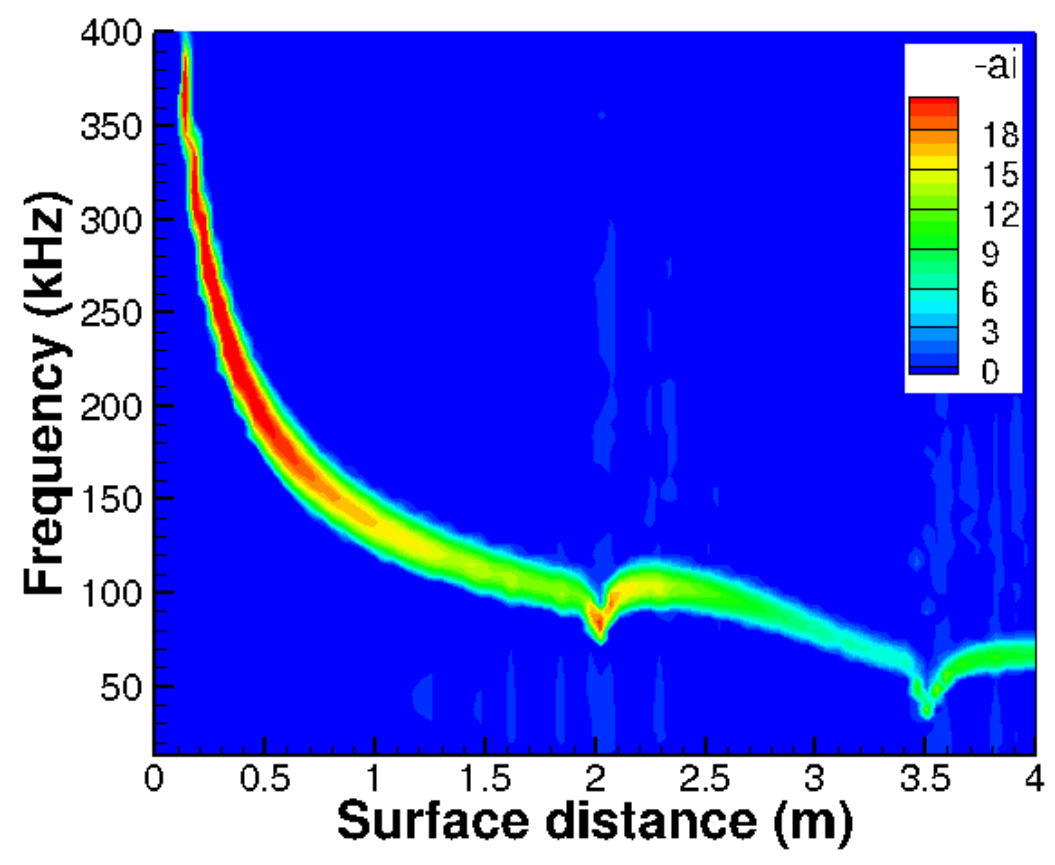

(b) Sample $664(w / h=4.74)$.

Figure 5.16: Effect of panel deformation on the second-mode disturbance frequency. Contour values greater than zero indicate unstable growth. 


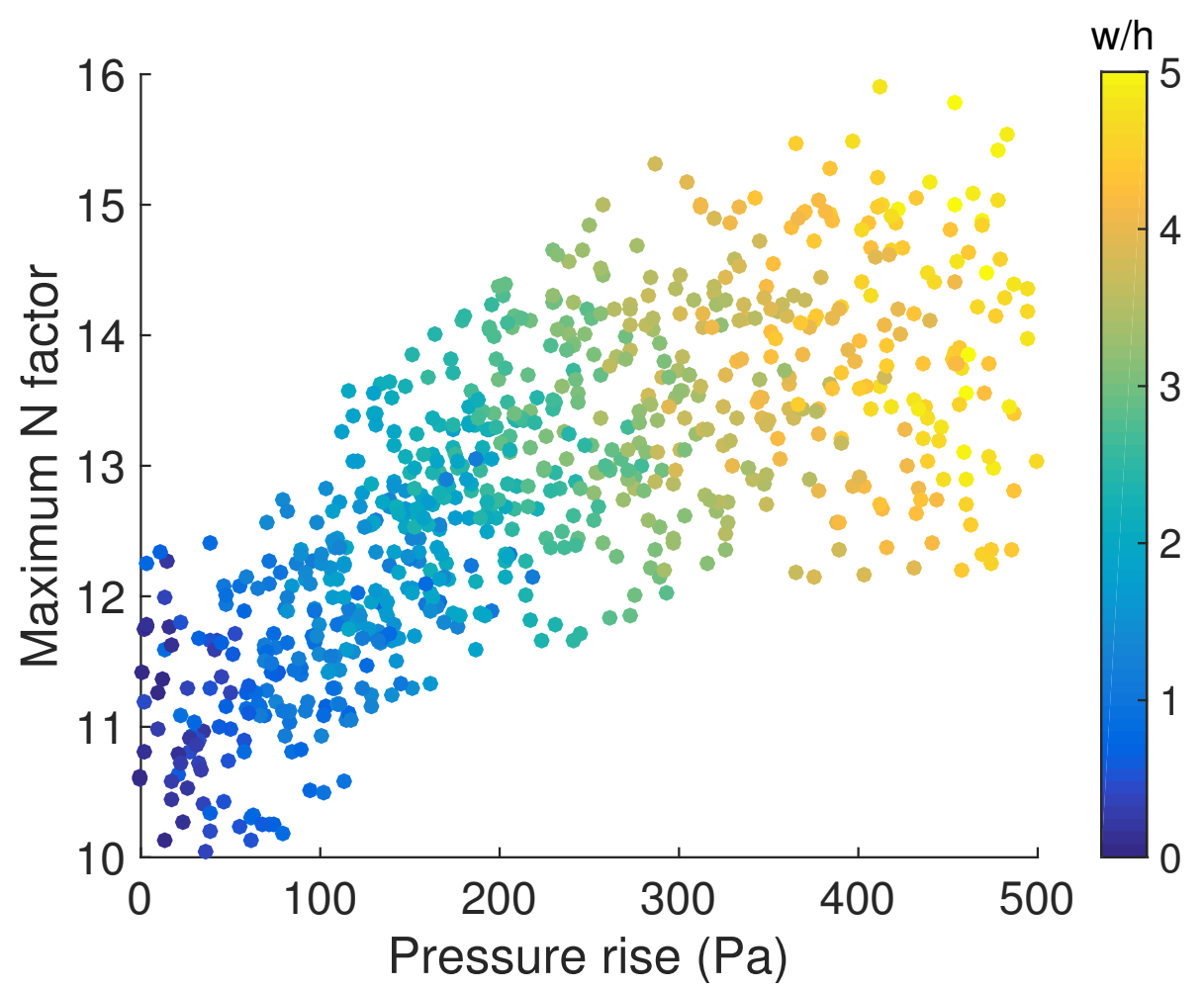

(a) Maximum $N$ factor.

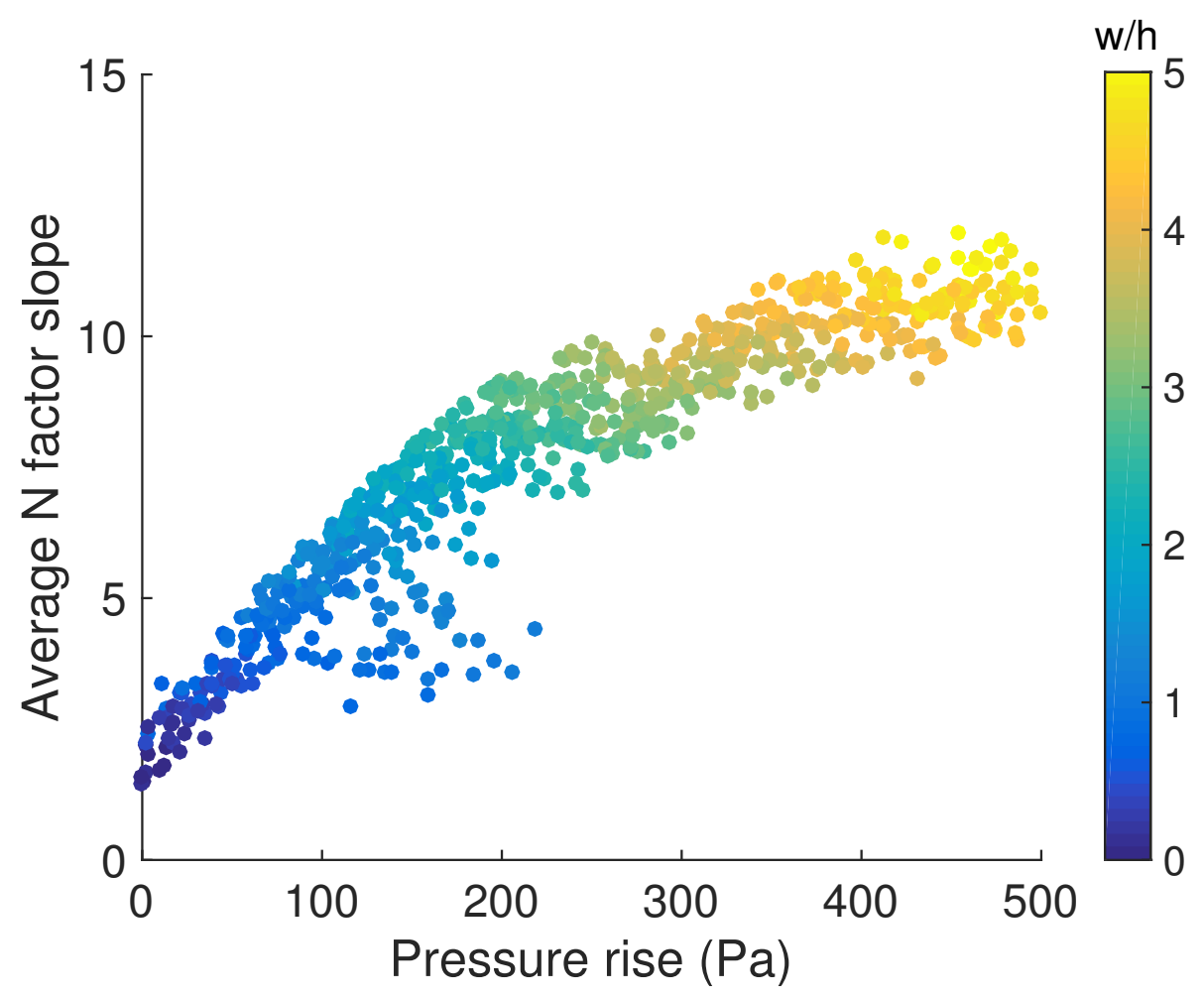

(b) Average $N$ factor slope.

Figure 5.17: Effect of adverse pressure gradient on $N$ factor growth. 


\subsubsection{Effect of Fluid Loading on Panel Response}

Thermal responses of the simply-supported and clamped panel subject to fully turbulent and transitional (fixed and time-varying) loading are compared in terms of the maximum (Fig. 5.18) and average (Fig. 5.19) temperature throughout the panel. The results in Fig. 5.18 indicate that transitional fluid loading produces maximum temperatures exceeding the turbulent prediction. Moreover, movement of the transition region results in larger variation in the maximum temperature for the clamped panel. A comparison of the transitional cases in Fig. 5.19 indicates that, during the $10 \mathrm{~s}$ of response, the forward movement of the transition region increases the average temperature of the simply-supported and clamped panels by $14 \mathrm{~K}$ and $12 \mathrm{~K}$.

Time histories of the simply-supported and clamped panel center displacement are shown in Fig. 5.20 for each loading condition. Note that the displacement is normalized by the panel thickness $h$. A comparison of the fixed and time-varying transitional responses in Fig. 5.20 indicates that, for either boundary condition, the forward movement of the transition region results in larger deformations. This is, in part, due to the increased average temperature of the panel which strongly influences the deformation [35]. For the simply-supported panel in Fig. 5.20.a, the increased displacement of the time-varying transitional response approaches the deformation predicted assuming fully turbulent flow. However, the same trend is not observed for clamped panel in Fig. 5.20.b as it buckles out of the flowfield when 


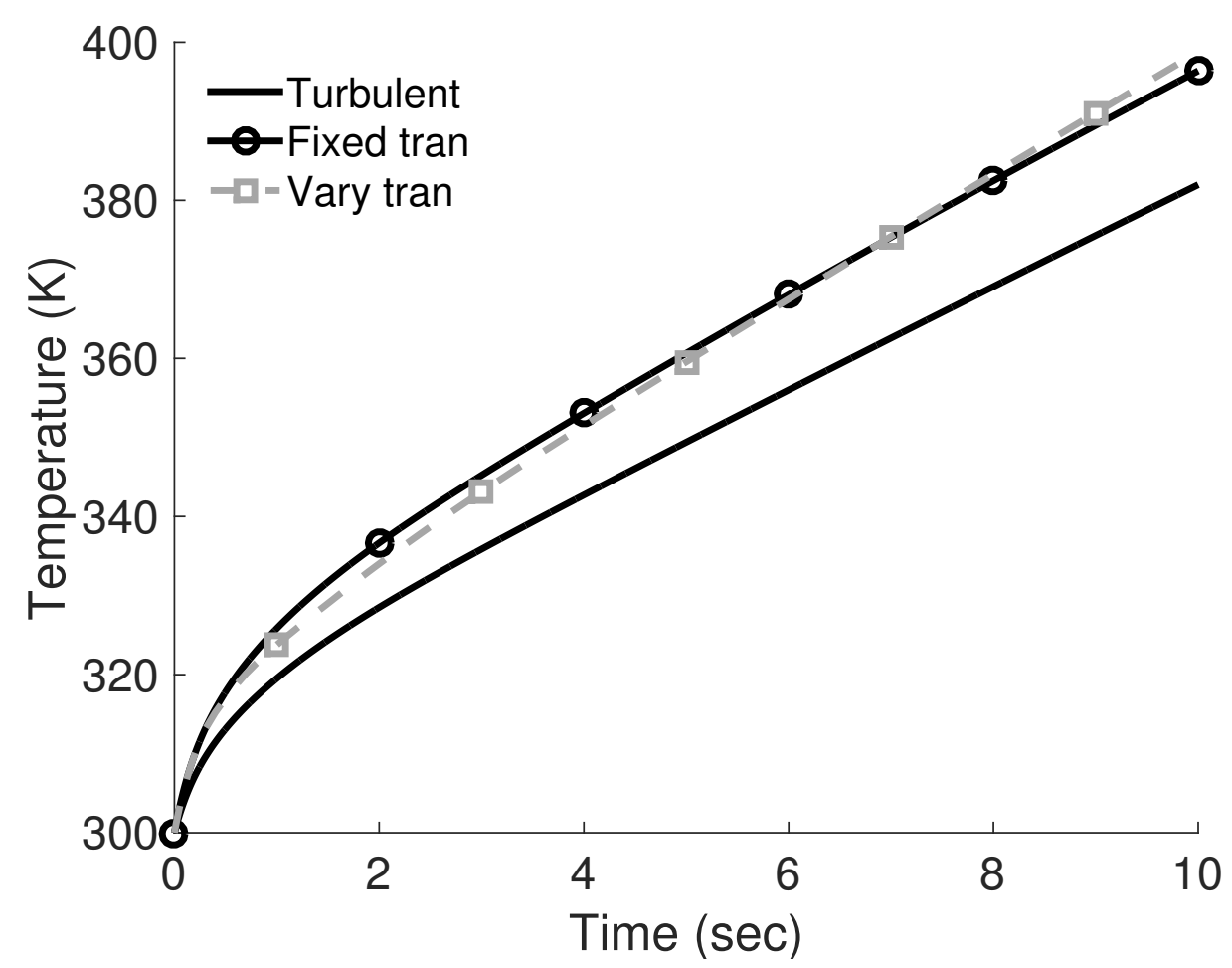

(a) Simply-supported panel.

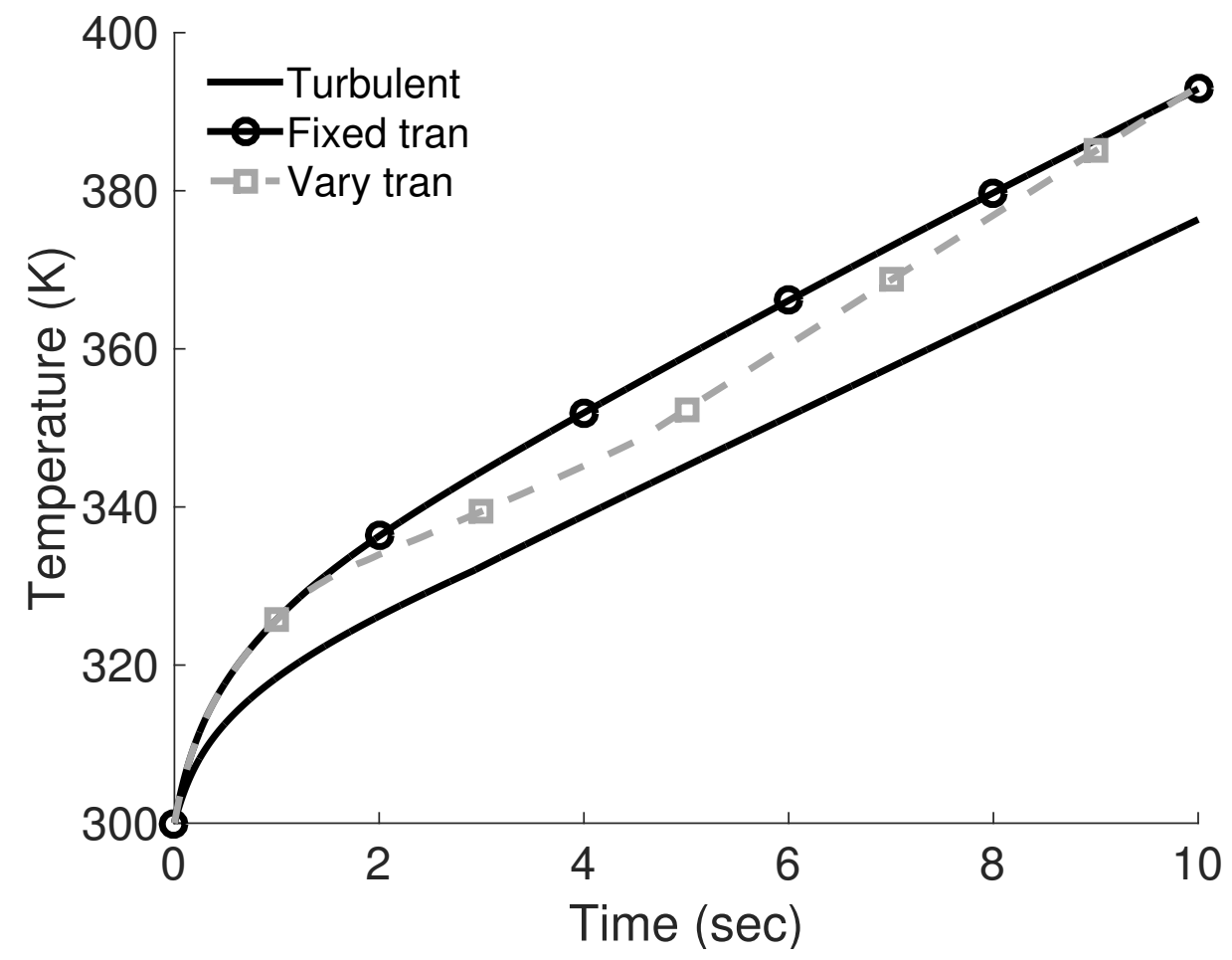

(b) Clamped panel.

Figure 5.18: Maximum panel temperature subject to fully turbulent and transitional (fixed and time-varying) loading. 


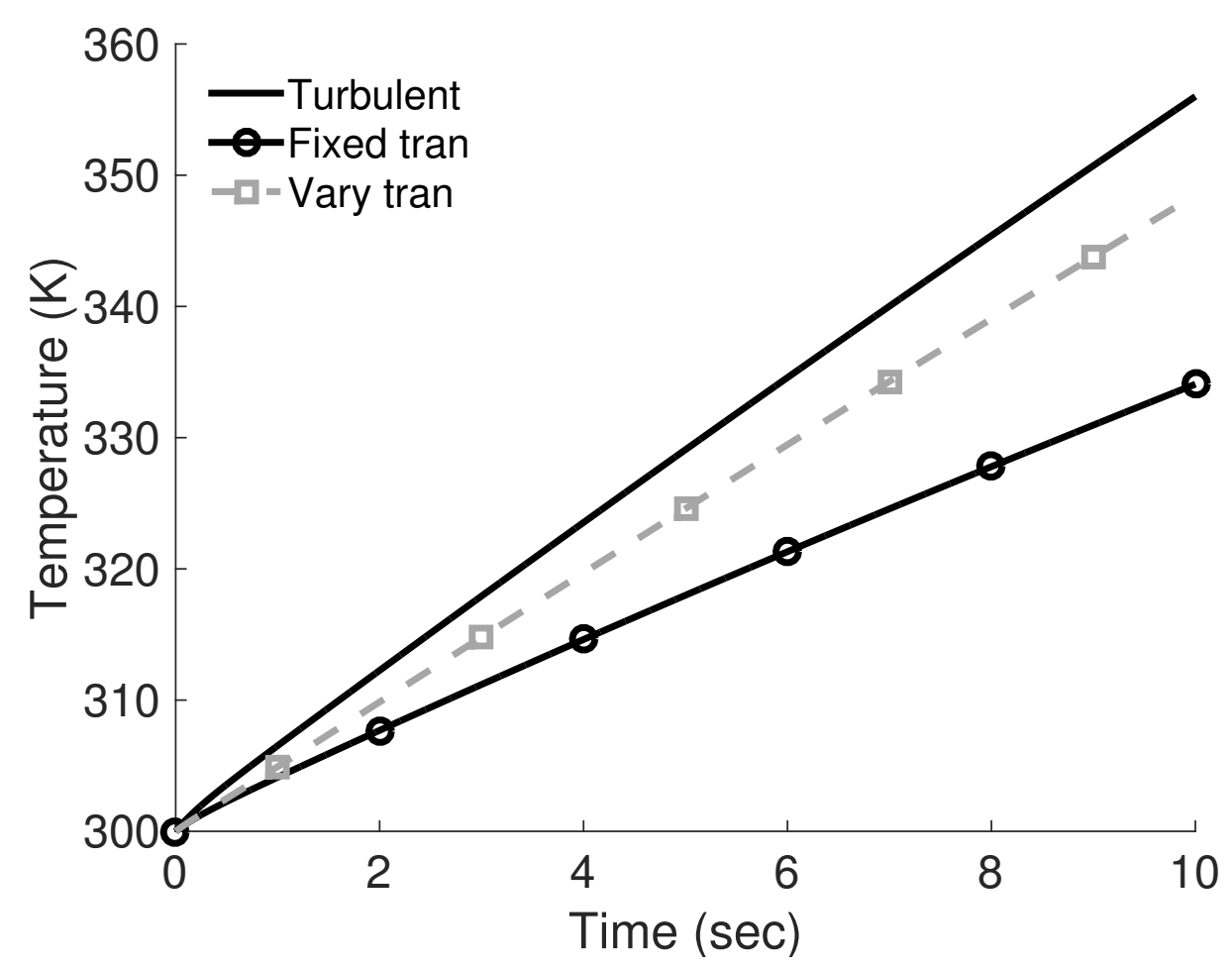

(a) Simply-supported panel.

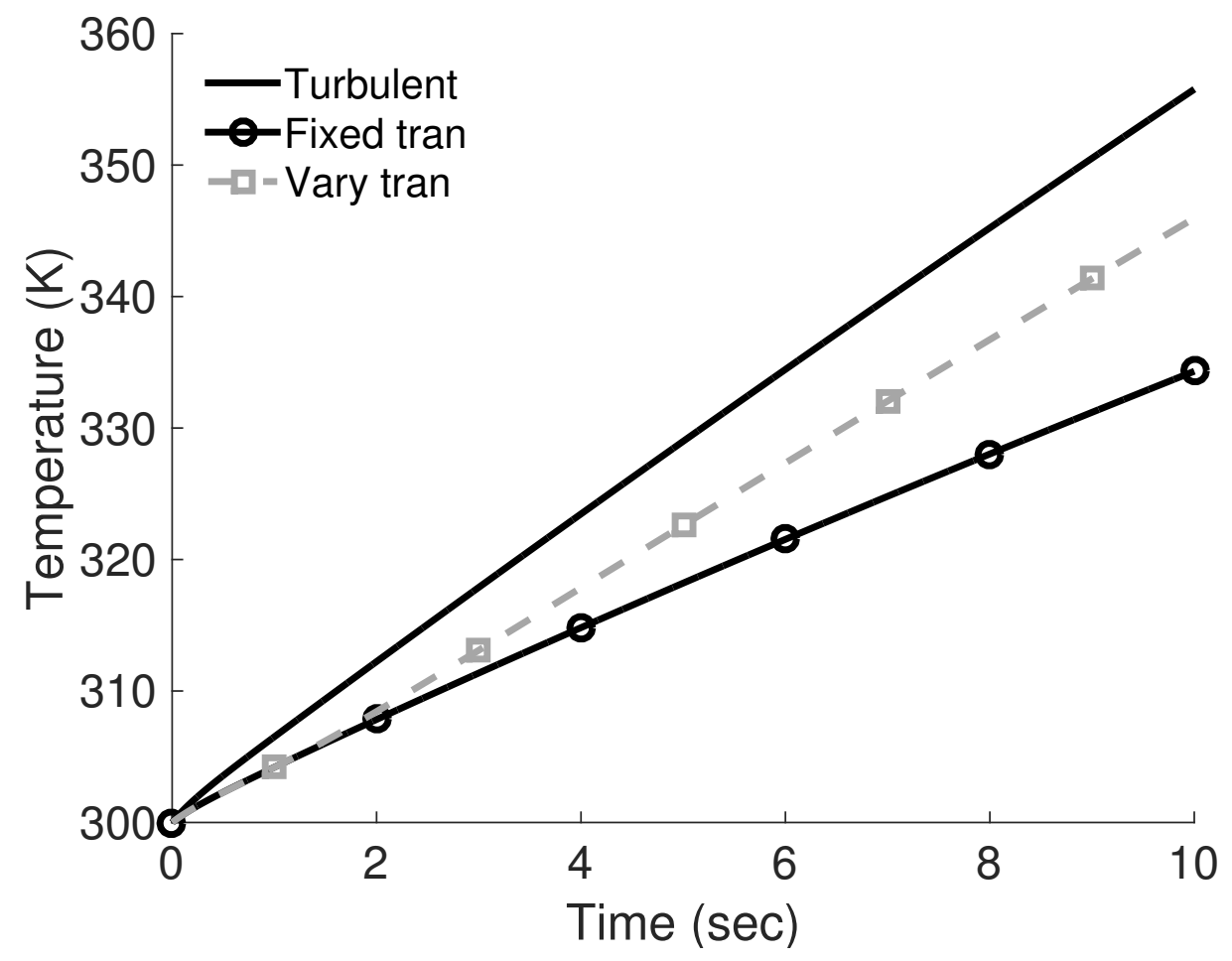

(b) Clamped panel.

Figure 5.19: Average panel temperature subject to fully turbulent and transitional (fixed and time-varying) loading. 
subject to turbulent loading. Unlike the simply-supported panel, the boundary conditions for the clamped panel in Eq. (2.57) are not dependent on the thermal moment. Therefore, the clamped panel is not inclined to buckle into the flowfield when heated from the top surface.

Snapshots of the simply-supported and clamped panel responses, due to both fixed and time-varying transitional loading, are provided in Figs. 5.21 and 5.22 in terms of the average through-thickness temperature, chordwise thermal gradient, thickness normalized displacement, and slope of the panel. A comparison of the thermal responses in Figs. 5.21.a and 5.22.a indicates that the movement of the transition region has a negligible effect (less than $4 \mathrm{~K}$ ) on the maximum temperature across the panel. However, the forward movement of the transition region greatly reduces the thermal gradient across both the simply-supported (Fig. 5.21.b) and clamped (Fig. 5.22.b) panels. This is clearly shown by the simply-supported panel responses at $10 \mathrm{~s}$ in Fig. 5.21.b, where the movement of the transition region reduces both the maximum $(66 \mathrm{~K} / \mathrm{m})$ and minimum $(82 \mathrm{~K} / \mathrm{m})$ thermal gradient as compared to the fixed transitional case. A similar reduction in the thermal gradient is shown for the clamped panel in Fig. 5.22.b.

A comparison of the displacements in Figs. 5.21.c and 5.22.c reveals that the forward movement of the transition region affects both the magnitude and spatial location of the peak deformation. The increase in magnitude is expected as the deformation is partially driven by the average temperature of the panel, which increases as the transition region moves upstream. The discrepancy in the location 


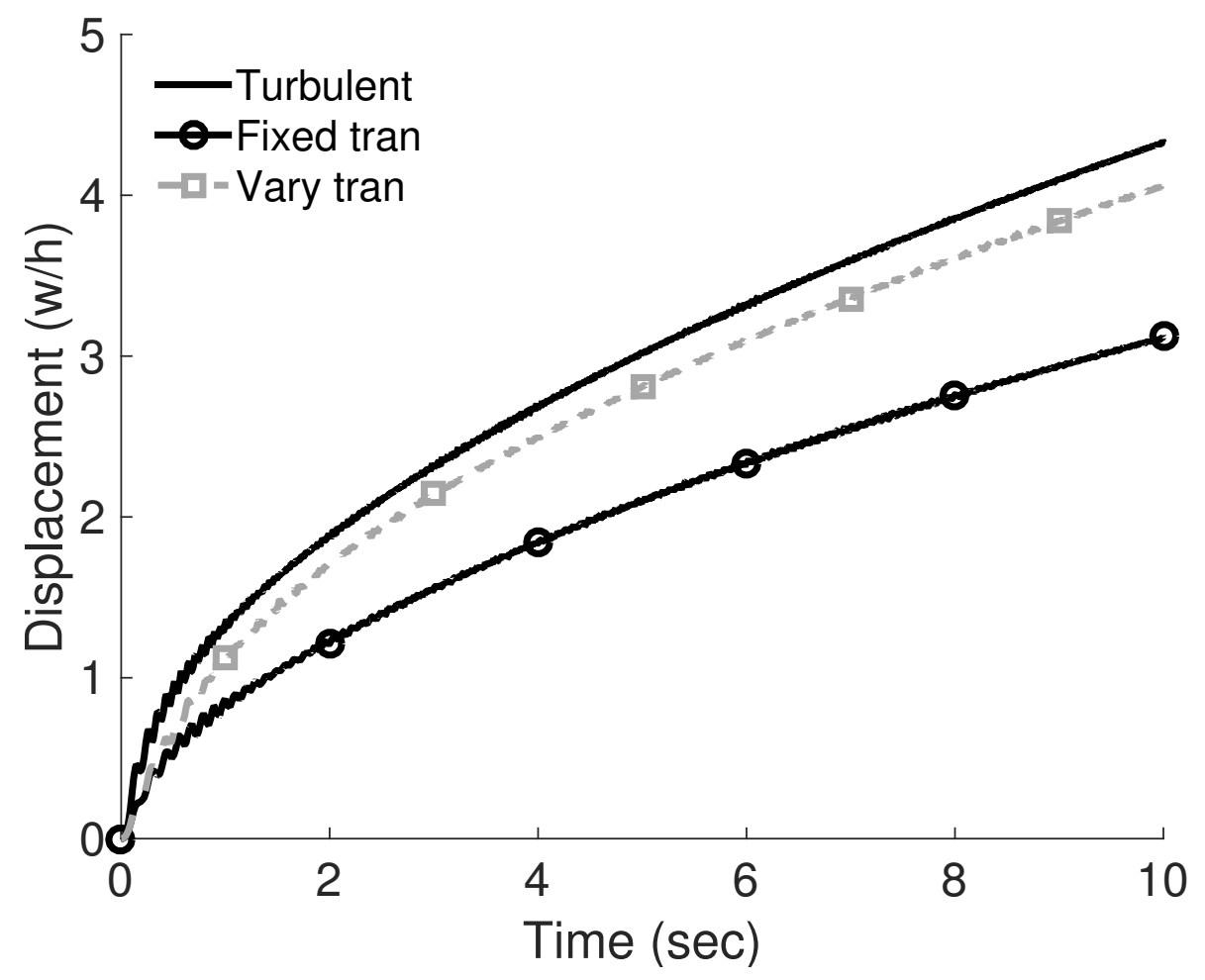

(a) Simply-supported panel.

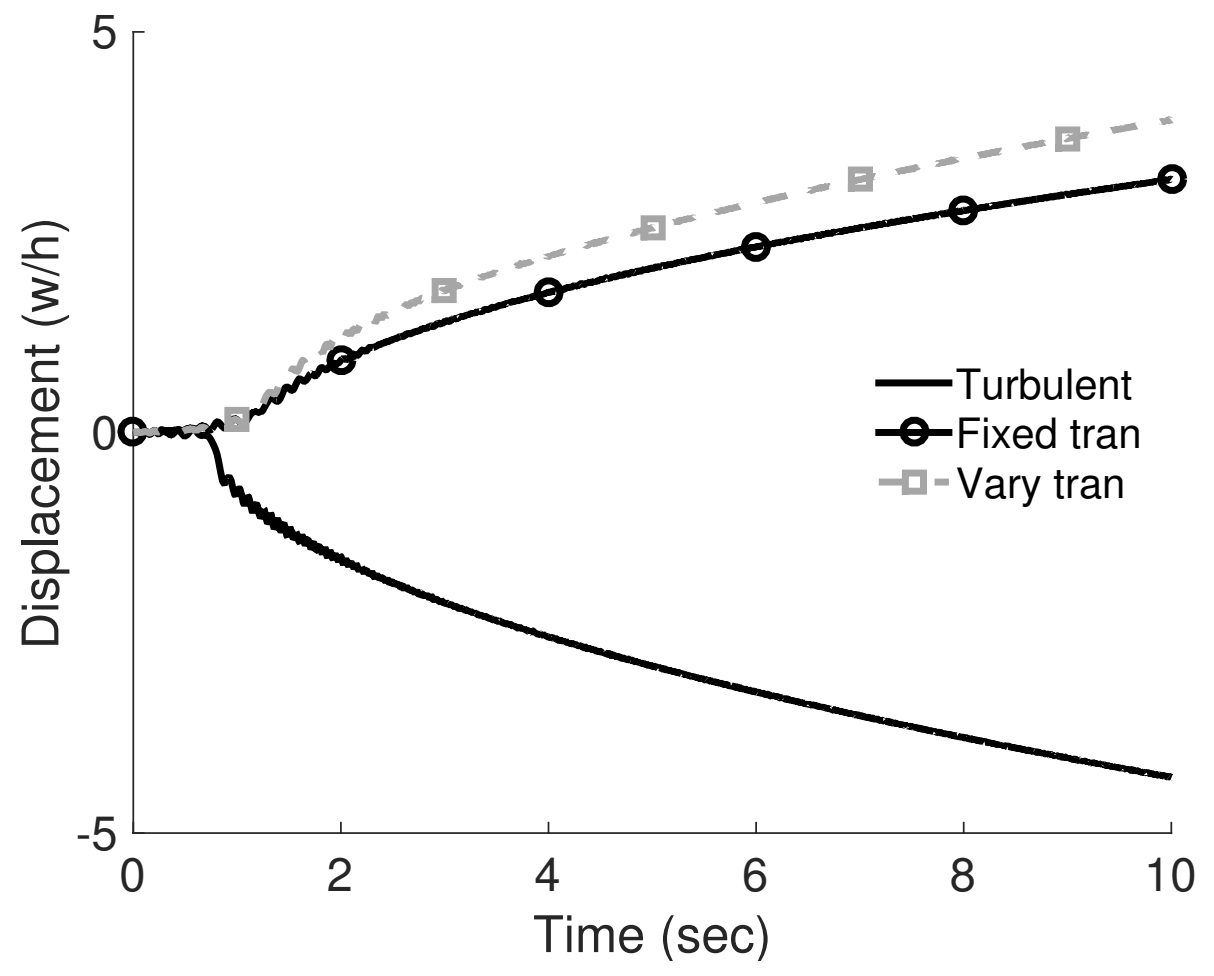

(b) Clamped panel.

Figure 5.20: Center displacement of panel subject to fully turbulent and transitional (fixed and time-varying) loading. 
of the peak deformation could be attributed to several factors: (1) deformation induced pressure, (2) panel stiffness, and (3) generalized thermal forces (in-plane force and bending moment). The magnitude of the deformation induced pressure is proportional to the panel slope. Therefore, as the panel deforms, the pressure increases on the forward portion, eventually shifting the peak displacement rearward. However, the results in Figs. 5.21.c-d and 5.22.c-d indicate that the peak displacement for the fixed transitional responses occurs further downstream even though the slope is smaller on the forward portion of the panel $(x / L \leq 0.36)$. This suggests that the variation in displacement in not entirely due to the aerodynamic pressure.

The discrepancy in displacement could result from spatial variation in the material properties (i.e., panel stiffness) due to the transitional aerodynamic heating. To examine this, the displacement of the simply-supported panel at $10 \mathrm{~s}$ is shown in Fig. 5.23, assuming fixed and time-varying transitional loading as well as temperature dependent " $f=f(T)$ " and constant " $f \neq f(T)$ " material properties. Note that the constant material properties correspond to a temperature of $350 \mathrm{~K}$. The close agreement for the constant and temperature-dependent responses indicates that the spatial variation in the material properties does not cause the disparity in displacement. Therefore, the difference in displacement is a result of the thermal forces acting on the panel.

In the structural equation of motion the average thermal force, $\overline{N_{T}}$, is integrated over the panel length. As a result of this, it only effects the magnitude of the dis- 


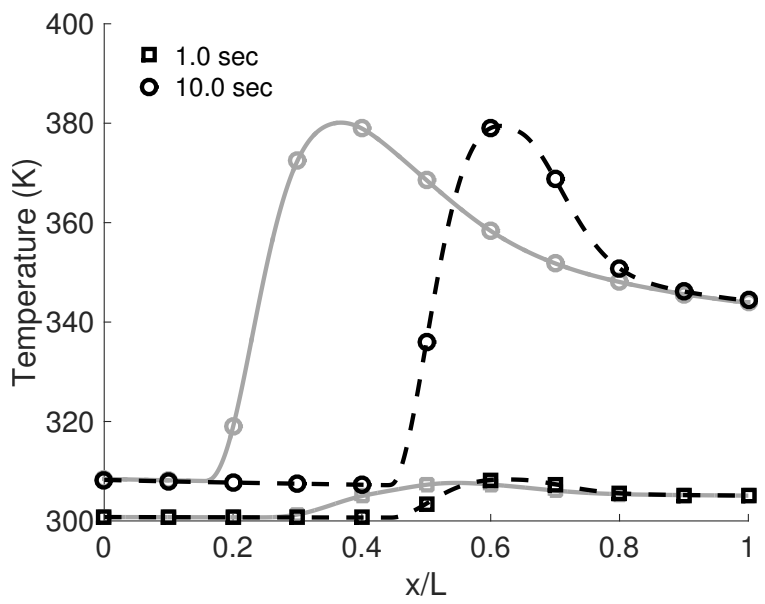

(a) Average through-thickness temperature.

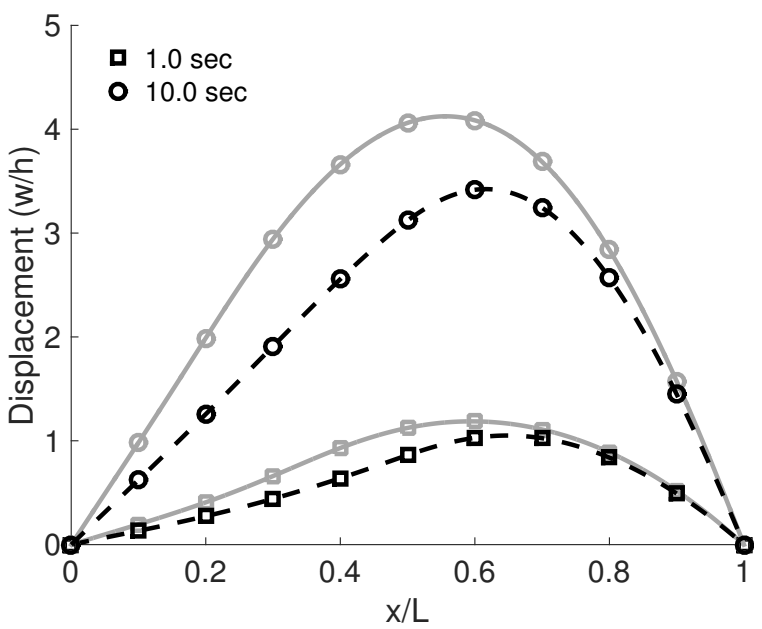

(c) Displacement.

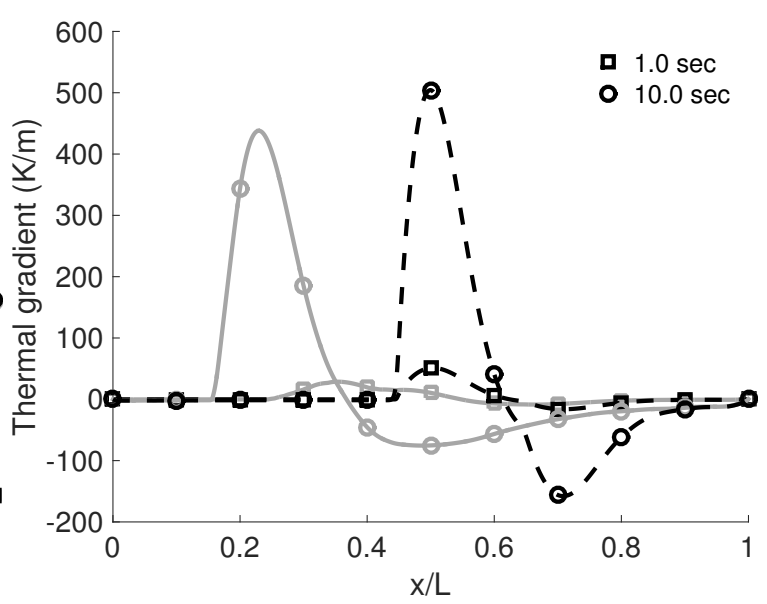

(b) Chordwise thermal gradient.

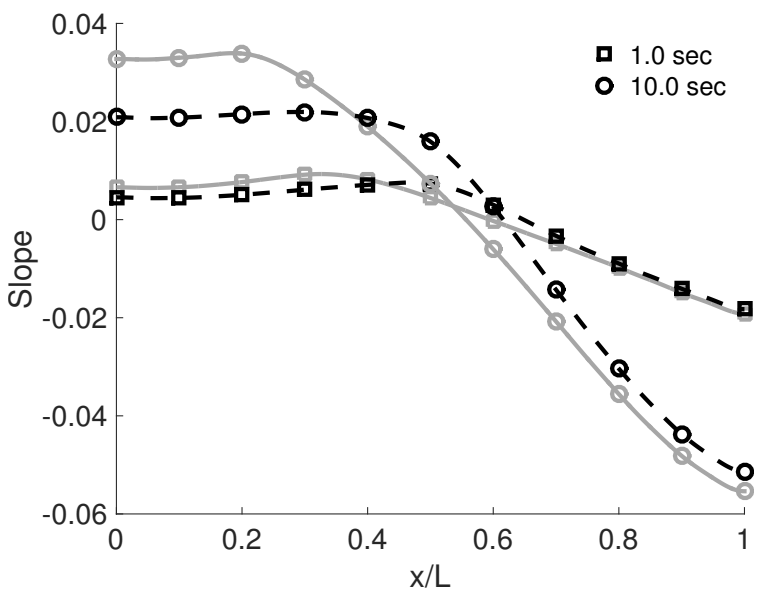

(d) Slope.

Figure 5.21: Variation in simply-supported panel response due to fixed (dashed lines) and time-varying (solid lines) transitional loading. 


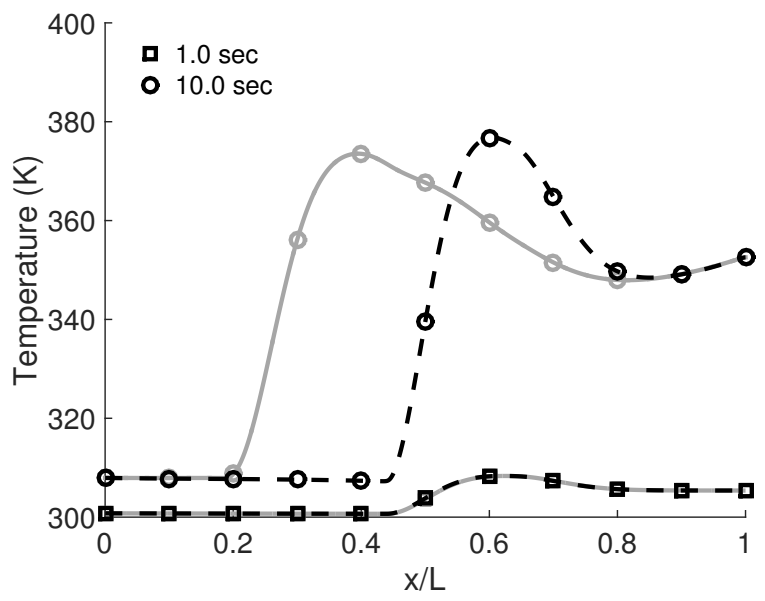

(a) Average through-thickness temperature.

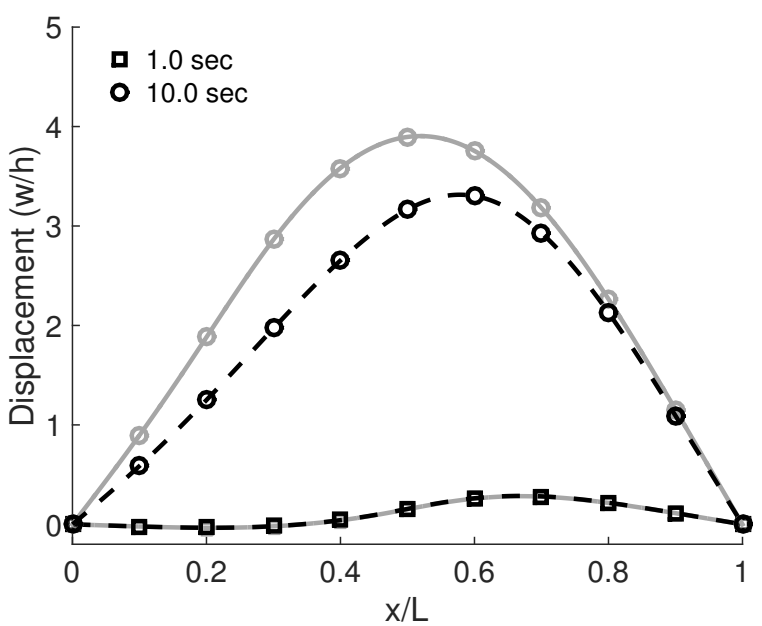

(c) Displacement.

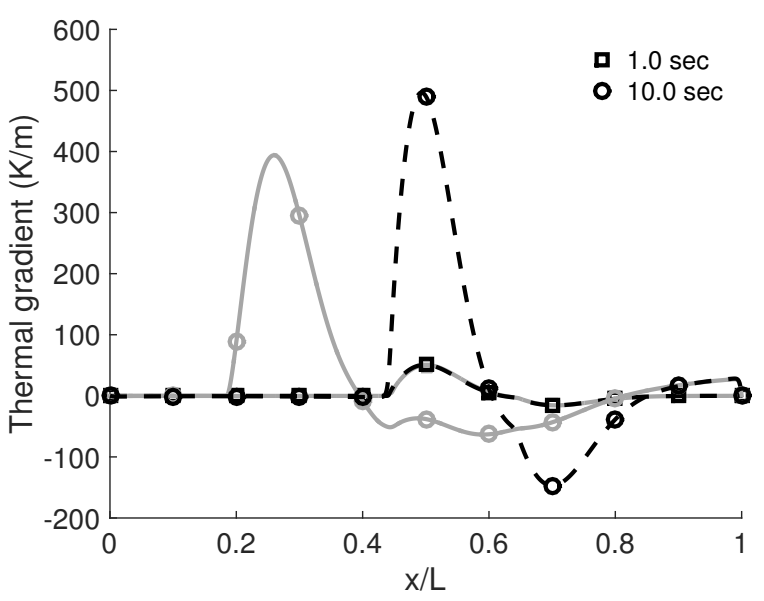

(b) Chordwise thermal gradient.

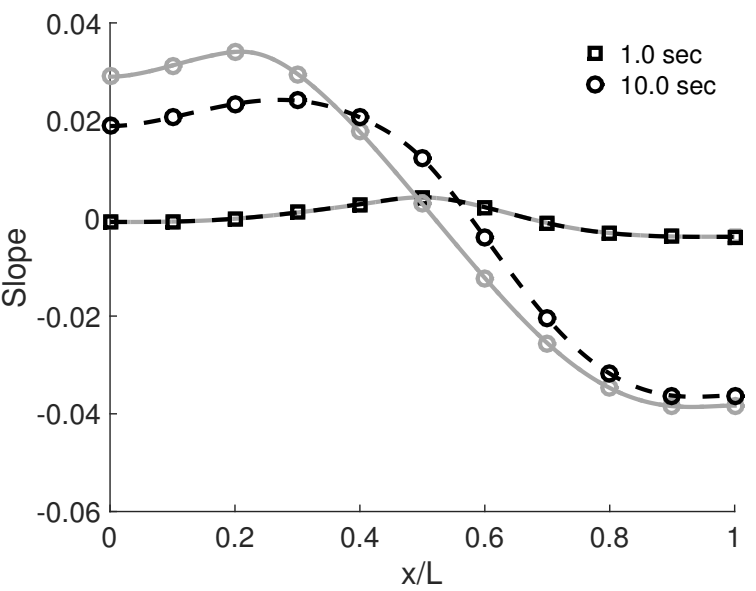

(d) Slope.

Figure 5.22: Variation in clamped panel response due to fixed (dashed lines) and time-varying (solid lines) transitional loading. 


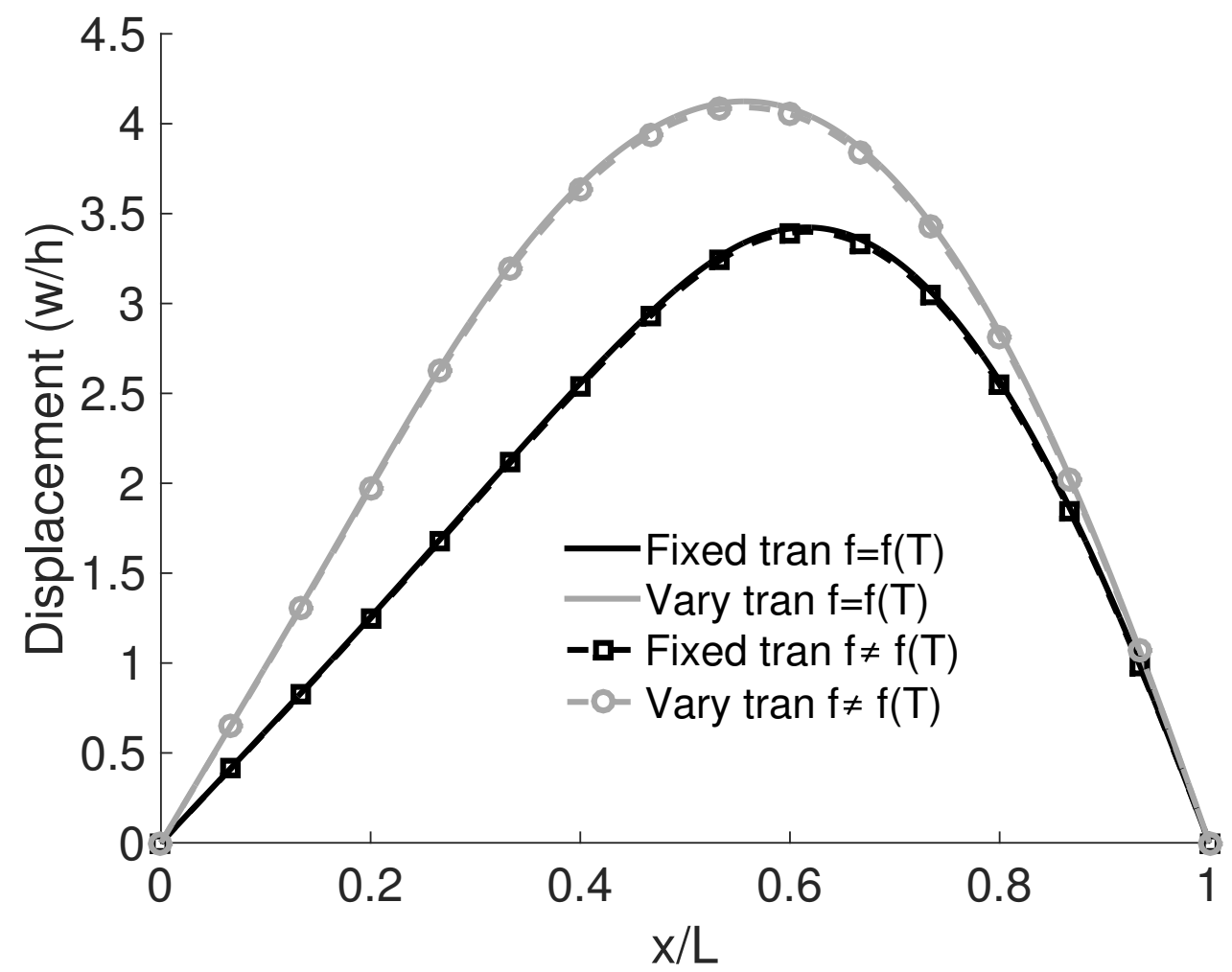

Figure 5.23: Variation in simply-supported panel displacement at $10 \mathrm{~s}$ due to material temperature dependence. 
placement and can not explain the shifted location of the peak displacement observed in the fixed transitional responses. However, the thermal bending moment enters the equation of motion as a distributed force $\left(\frac{\partial^{2} M_{T}(x, t)}{\partial x^{2}}\right)$ that varies along the panel length. The thermal bending moment at $10 \mathrm{~s}$ is shown in Fig. 5.24.a for the simply-supported panel subject to fully turbulent and transitional (fixed and timevarying) loading. For the fixed transitional response, the thermal moment is small over the forward portion of the panel $(x / L \leq 0.44)$ due to the region of laminar flow. This reduction in the thermal moment directly correlates to the decreased displacement over the forward panel causing the difference between the fixed and time-varying transitional responses. The impact of the thermal bending moment is further illustrated by the displacement profiles in Fig. 5.24.b. A comparison of the displacements indicate that, for similar peak deformation, the characteristic shape of the panel is different due to the spatial variation in the thermal bending moment.

To further demonstrate the importance of the thermal moment, steady state panel responses are obtained assuming prescribed step function thermal moments, as shown in Fig. 5.25.a. The magnitude and location of the rise in thermal moment closely match that of the transitional responses in Fig. 5.24.a. The deformations in Fig. 5.24.b-d are obtained assuming the thermal moments in Fig. 5.24.a. However, for the responses in Fig. 5.24.b the average temperature of the panel is $10 \mathrm{~K}$ less than that in Fig. 5.24.c-d. Additionally, the responses in Fig. 5.24.b-c assume no pressure load while the response in Fig. 5.24.d has an applied pressure corre- 


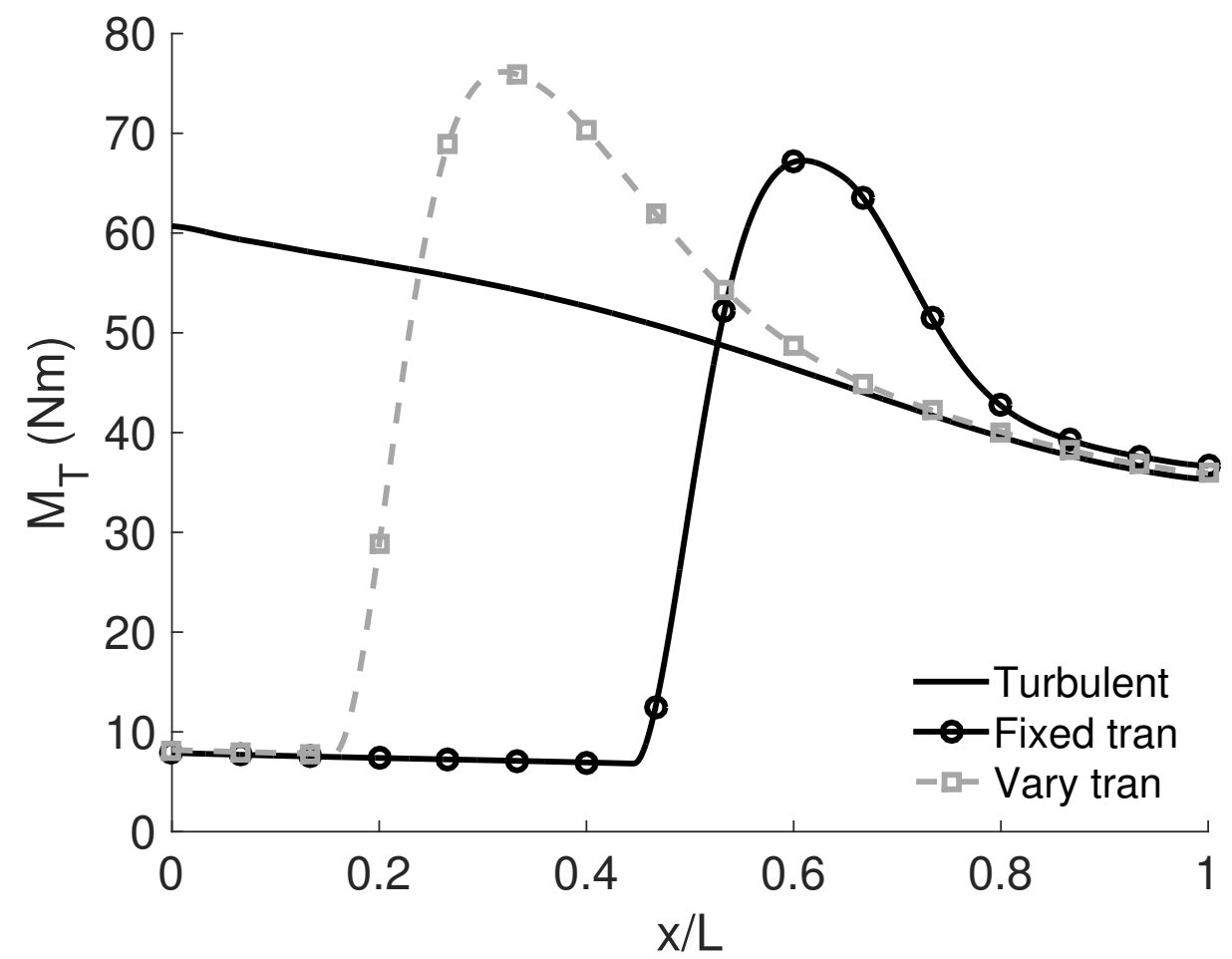

(a) Thermal moment.

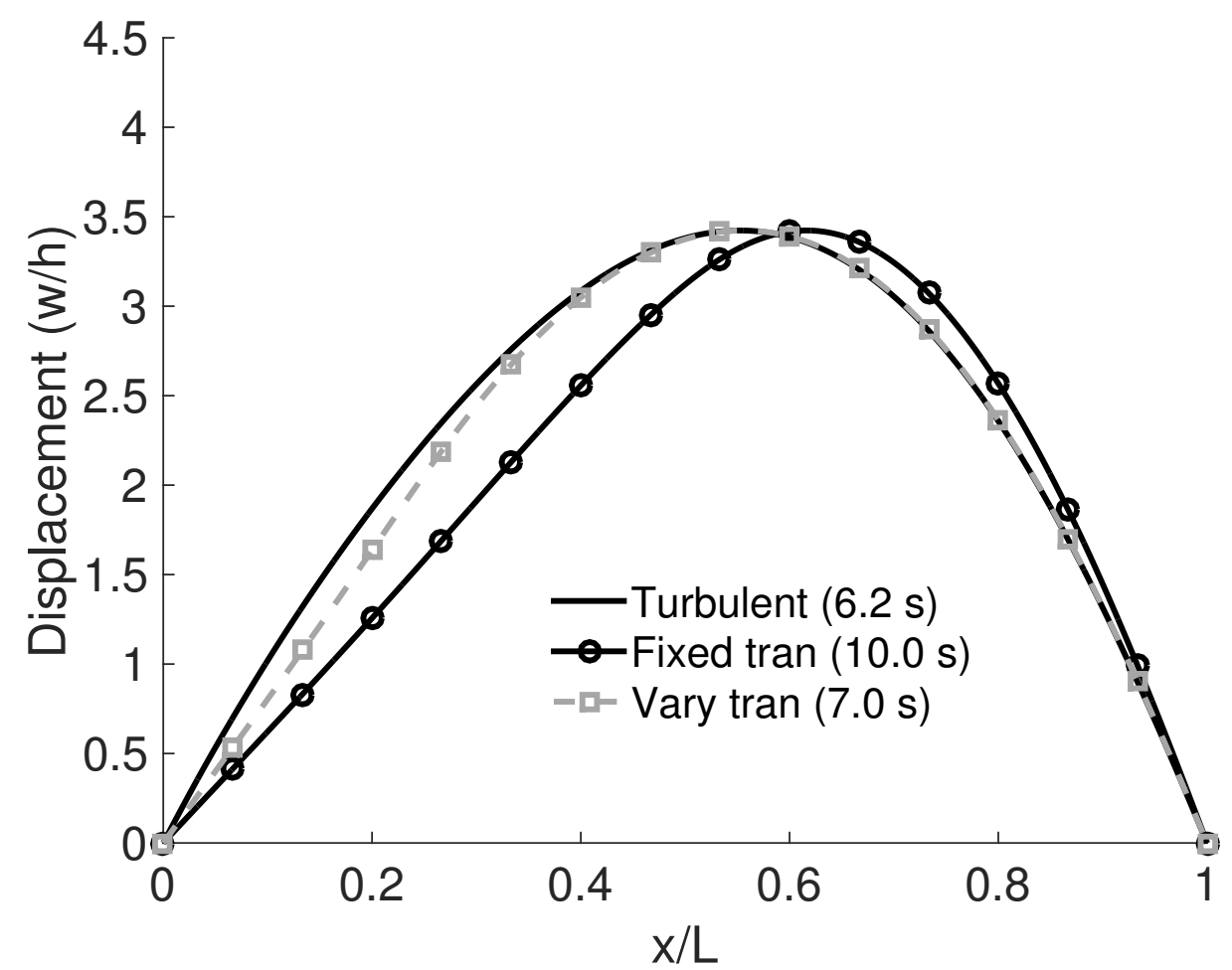

(b) Variation in panel response due to loading.

Figure 5.24: Importance of thermal moment on simply-supported panel response. 
sponding to the time-varying transitional response of the simply-supported panel at 10 s. A comparison of Fig. 5.24.b-c indicates that while the difference in the average in-plane thermal force alters the magnitude of the deformation, the impact of the thermal moment remains the same. In either case, the thermal moment rise at $x / L=0.45$ results in decreased displacement over the forward portion of the panel and a peak that is shifted downstream. Examination of Fig. 5.24.d indicates that inclusion of the pressure load further reduces the deformation on the forward portion of the panel, resulting in a displacement profile resembling that of the preflutter response.

Steady state responses of the simply-supported and clamped panel, assuming no pressure loading, are provided in Fig. 5.26 for step function thermal moments with rise locations between 65 and $75 \%$ of the panel length. A comparison of the displacements in Figs. 5.26.a and 5.26.b indicates that the clamped panel is more sensitive to the location of the thermal moment rise than the simply-supported panel. For each thermal moment, the simply-supported panel deforms into the flowfield in a predominantly first mode deformation (Fig. 5.26.a). However, for the clamped panel in Fig. 5.26.b the $10 \% L$ variation in the thermal moment rise results in distinctly different deformations resembling the $1^{s t}, 2^{\text {nd }}$, and $4^{\text {th }}$ free vibration modes of the panel. As previously stated, the clamped boundary conditions have no explicit dependence on the thermal moment. This allows for a broader range of deformations depending on the location of the rise in thermal moment. The step function thermal moment is an approximation of the thermal response of the 


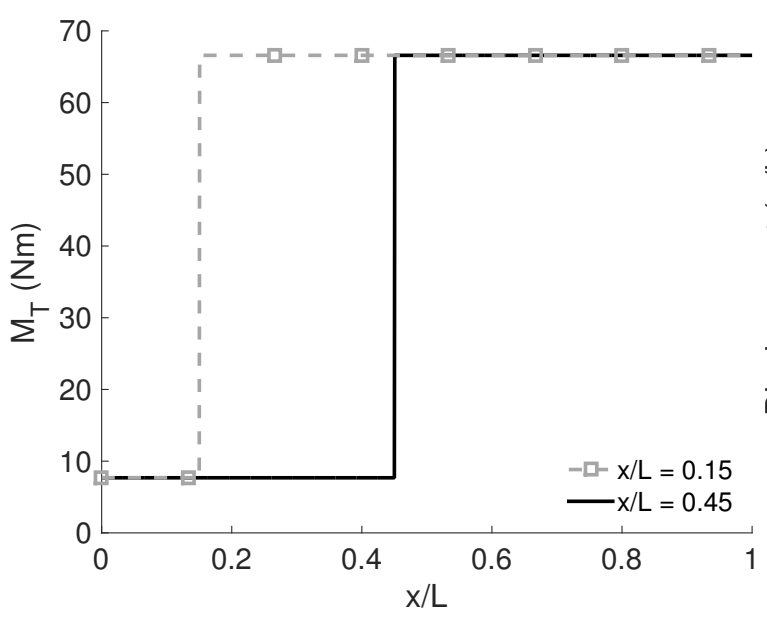

(a) Step function thermal moment.

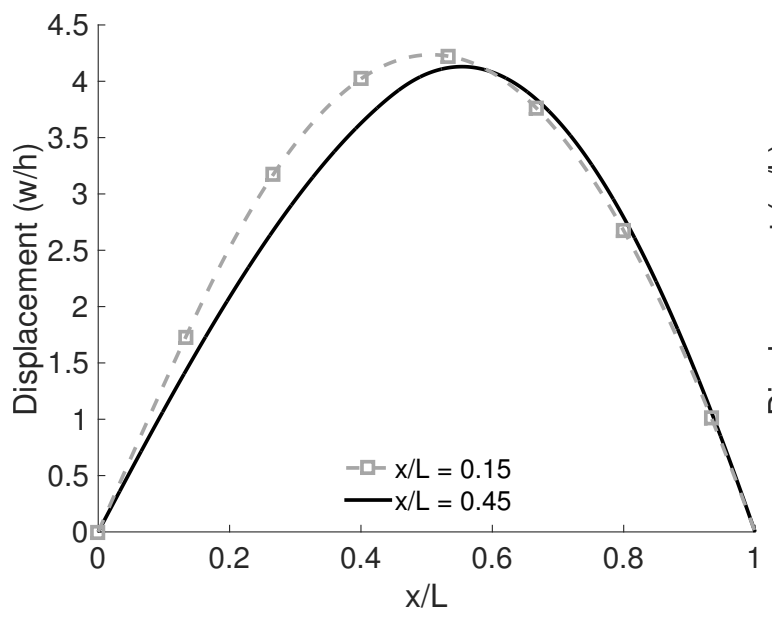

(c) No pressure and high $T_{\text {avg }}$.

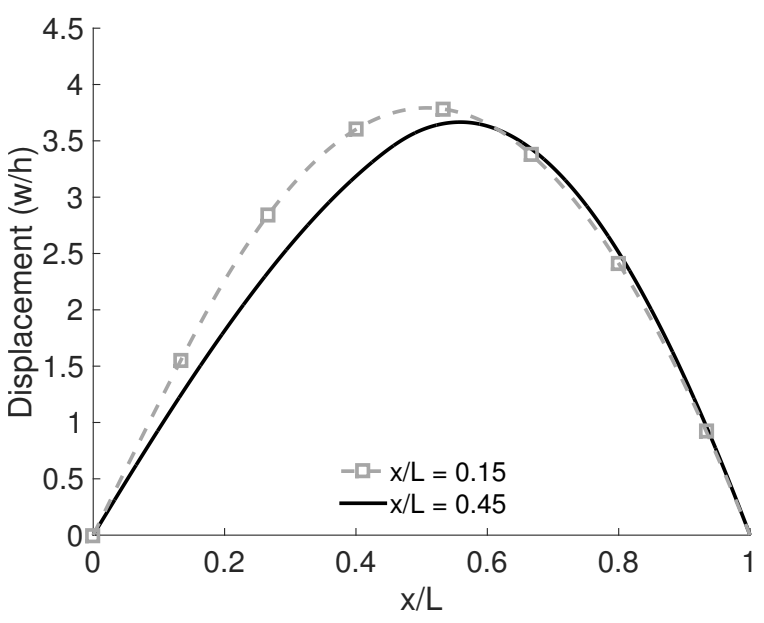

(b) No pressure and low $T_{\text {avg }}$.

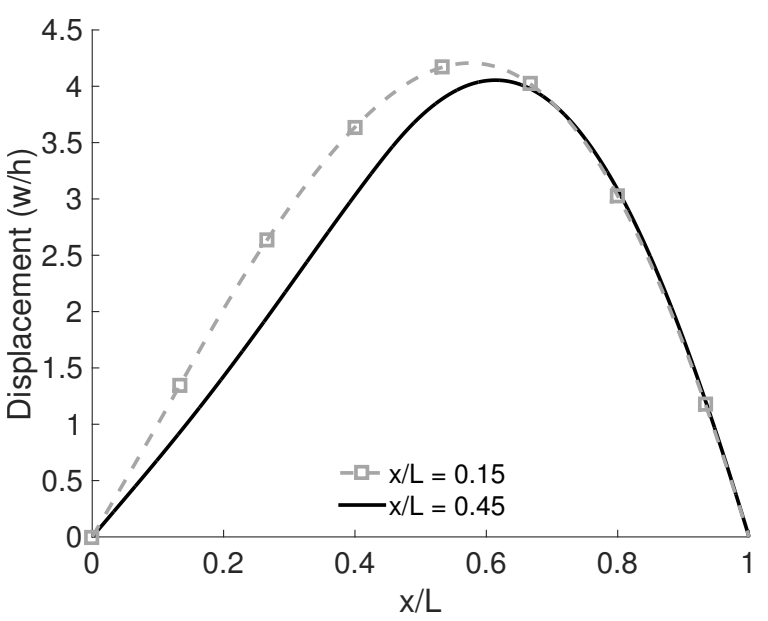

(d) Pressure load and high $T_{\text {avg }}$.

Figure 5.25: Impact of step function thermal moment on simply-supported panel displacement. 
panel under transitional loading. Therefore, the results in Fig. 5.26.b reveal that the clamped panel is highly sensitive to the transition onset location.

\subsubsection{Structural Sensitivity to the Transitional $N$ Factor}

Throughout this work, transition onset is predicted using the semi-empirical $e^{N}$ method. As stated in Section 2.1.3, the $N$ factor at transition can vary from one application to another. This variability is well documented and significant work has examined the variation in $N$ factor due to freestream turbulence intensity [73, 165] and surface roughness [166]. For aerothermoelastic analysis, the variability in the transitional $N$ factor introduces uncertainty into the predicted structural response.

The sensitivity of the structural response to the transitional $N$ factor is explored here by obtaining panel responses assuming transition onset occurs at $N=8.8,10$, and 10.2. Note that the transitional $N$ factor is restricted to a range of 8.8-10.2 to ensure transition occurs over the panel location and allows for $10 \mathrm{~s}$ of simplysupported panel response before the surrogate bounds are exceeded. The $N$ factor of 8.8 ensures transition occurs on the panel and is near the lower bound typically observed for smooth bodies in quiet (i.e., freestream) flow. The upper limit of $N=10.2$ demonstrates how a small variation in the transitional value of $N$ can alter the response of the structure. The movement of the transition onset location corresponding to each assumed $N$ factor is shown in Fig. 5.27 for the simply- 


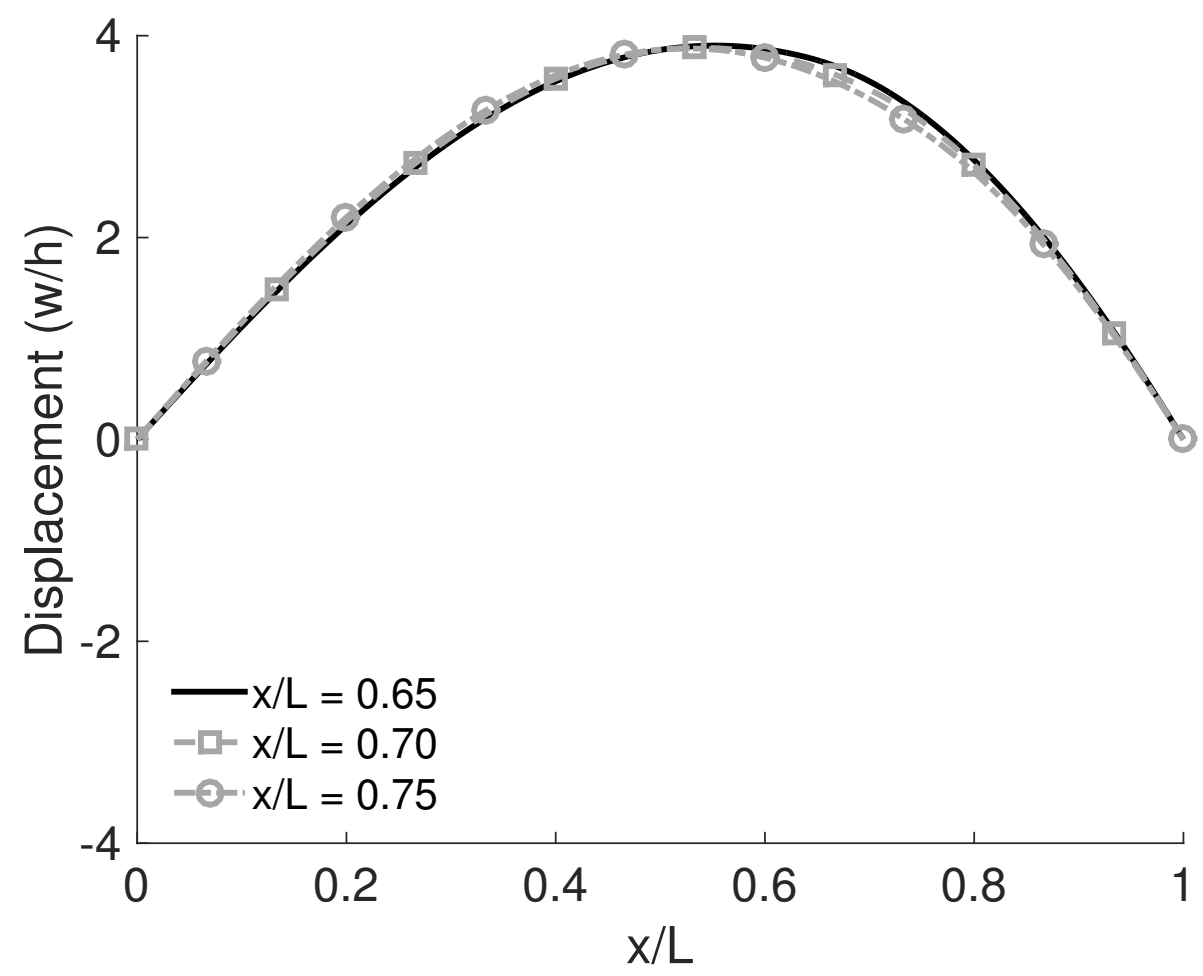

(a) Simply-supported panel.

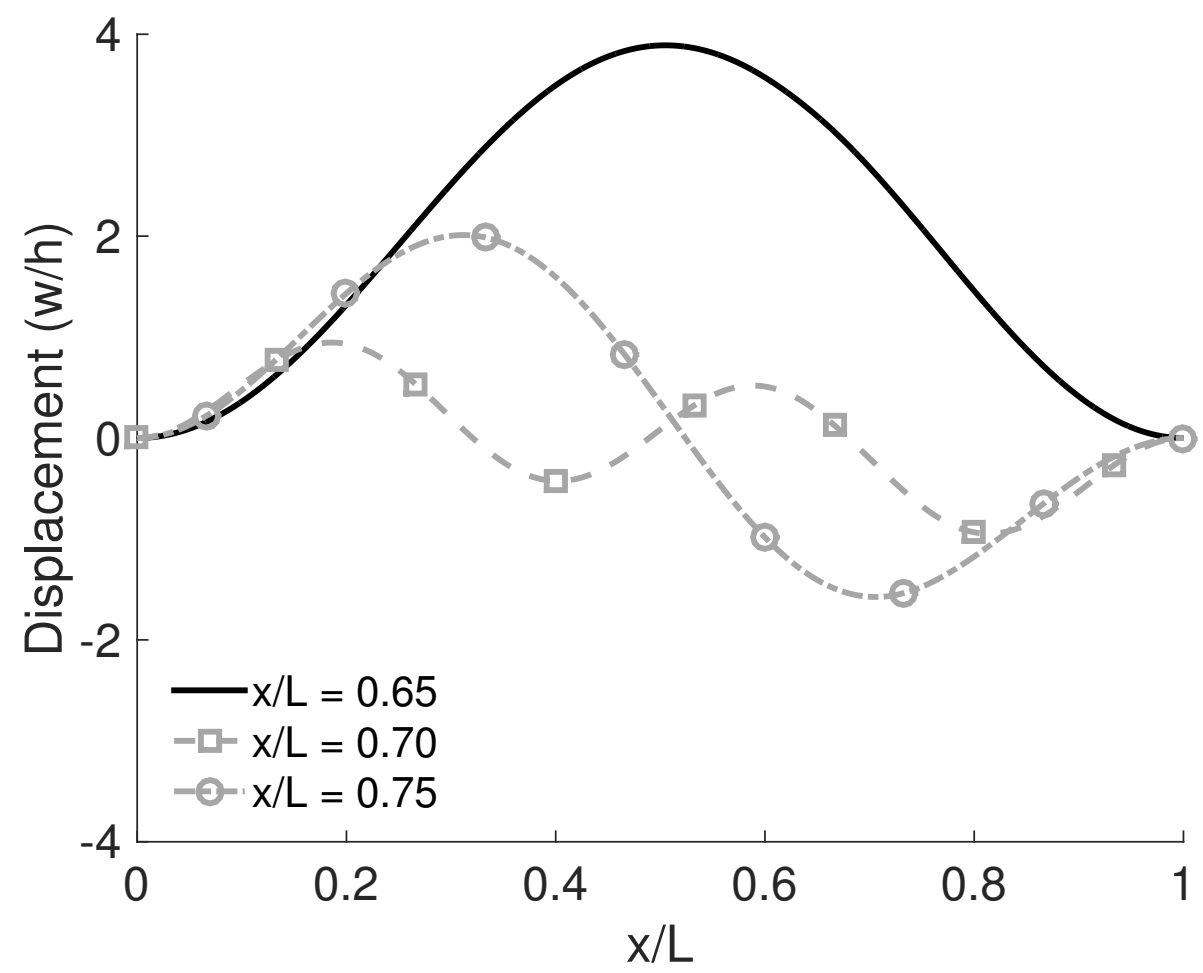

(b) Clamped panel.

Figure 5.26: Impact of step function thermal moment on panel displacement. No pressure loading. 
supported and clamped panel. Examination of Fig. 5.27.a indicates that, for the simply-supported panel, the largest difference in onset location $(39.0 \% L)$ occurs at the beginning of the simulation. The variation in the predicted onset location decreases in time, falling below the maximum surrogate error of $10.3 \% L$ by $4.1 \mathrm{~s}$ and achieving a minimum value of $7.5 \% L$ by $10 \mathrm{~s}$. This trend is not observed for the clamped panel in Fig. 5.27.b as the onset location remains fixed at 50.8\% $L$ for transition at $N=10.2$. The constant onset location is a result of the panel deflecting out of the flowfield, into a configuration that moves the $N=10.2$ predicted onset location downstream. As relaminarization of the boundary layer is not accounted for in this work, the transition onset location remains fixed at the most upstream prediction.

The structural response of the simply-supported and clamped panel is provided in Figs. 5.28 and 5.29 in terms of the mid-chord displacement and average temperature. A comparison of the $N=10$ and 10.2 responses in Figs. 5.28.a and 5.29.a indicates that the simply-supported panel is largely insensitive to small perturbations about the assumed transitional $N$ factor. Moreover, varying the $N$ factor from 8.8 to 10 only results in maximum displacement and temperature differences of $36 \% h$ at $0.35 \mathrm{~s}$ and $5.4 \mathrm{~K}$ at $10 \mathrm{~s}$. The clamped panel is more sensitive to the variation in $N$ factor from 8.8 to 10, resulting in maximum displacement and temperature differences of $88 \% h$ at $1.07 \mathrm{~s}$ (Fig. 5.28.b) and $8.0 \mathrm{~K}$ at $10 \mathrm{~s}$ (Fig. 5.29.b). Furthermore, the $N=10$ and 10.2 displacements in Fig. 5.28.b highlight the sensitivity of the clamped panel response to the transition onset location. As shown in 


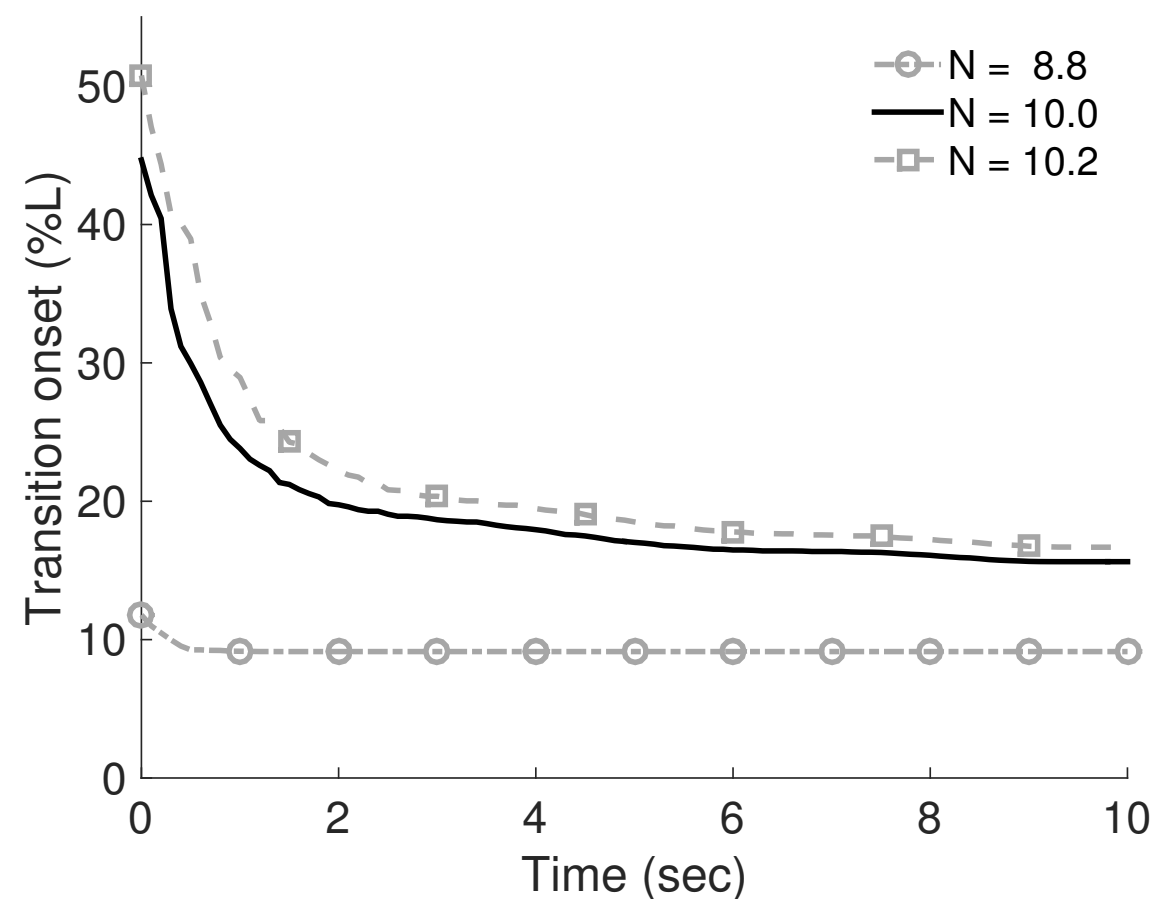

(a) Simply-supported panel.

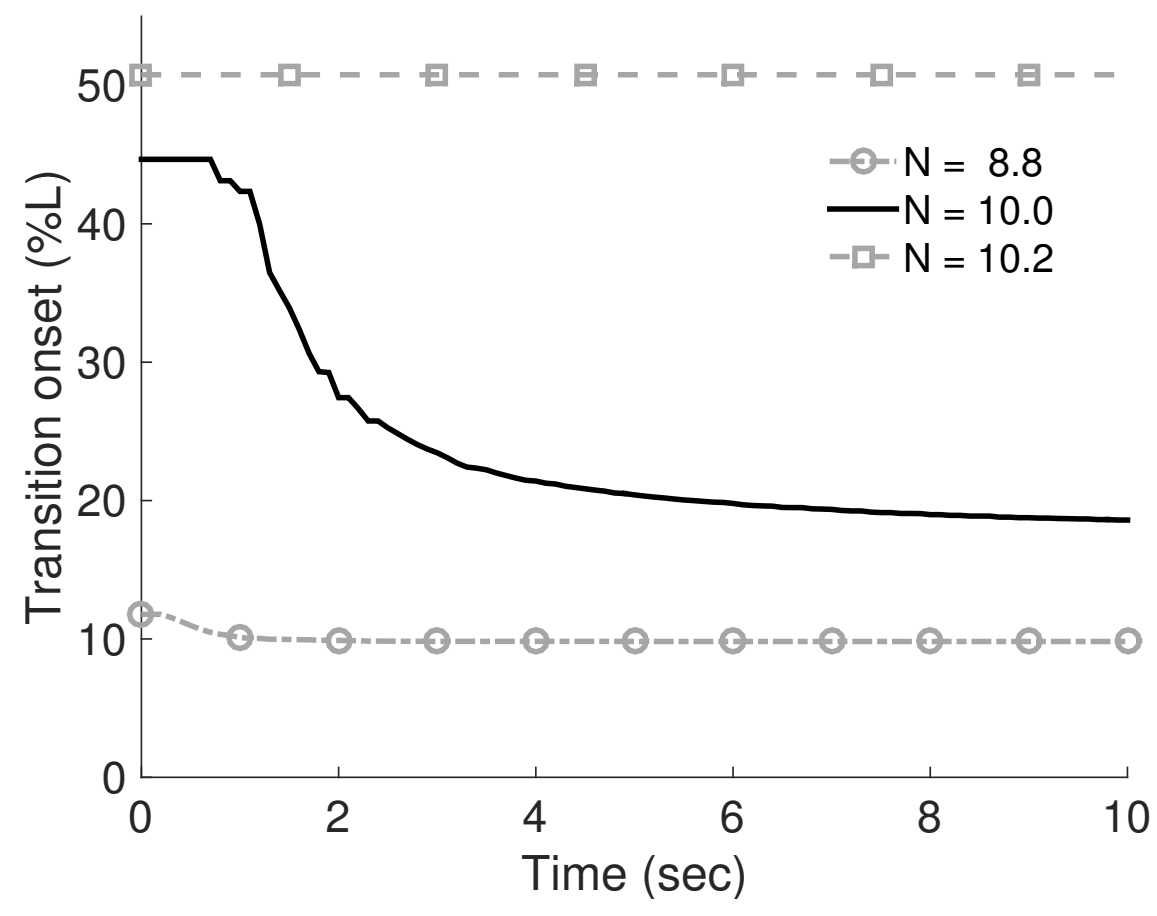

(b) Clamped panel

Figure 5.27: Movement of the transition front for variation in transitional $N$ factor. 
Fig. 5.27.b, the increase in $N$ of 0.2 moves the initial onset location downstream by $6.1 \% L(9.17 \mathrm{~cm})$. This slight variation in the onset location alters the aerothermodynamic loads, causing the clamped panel to buckle out of the flowfield. It should be noted that the deformation of the clamped panel for the $N=10.2$ response exceeds the surrogate bounds at $1.25 \mathrm{~s}$. However, the panel deformation deviates from the $N=10$ response prior to this time. 


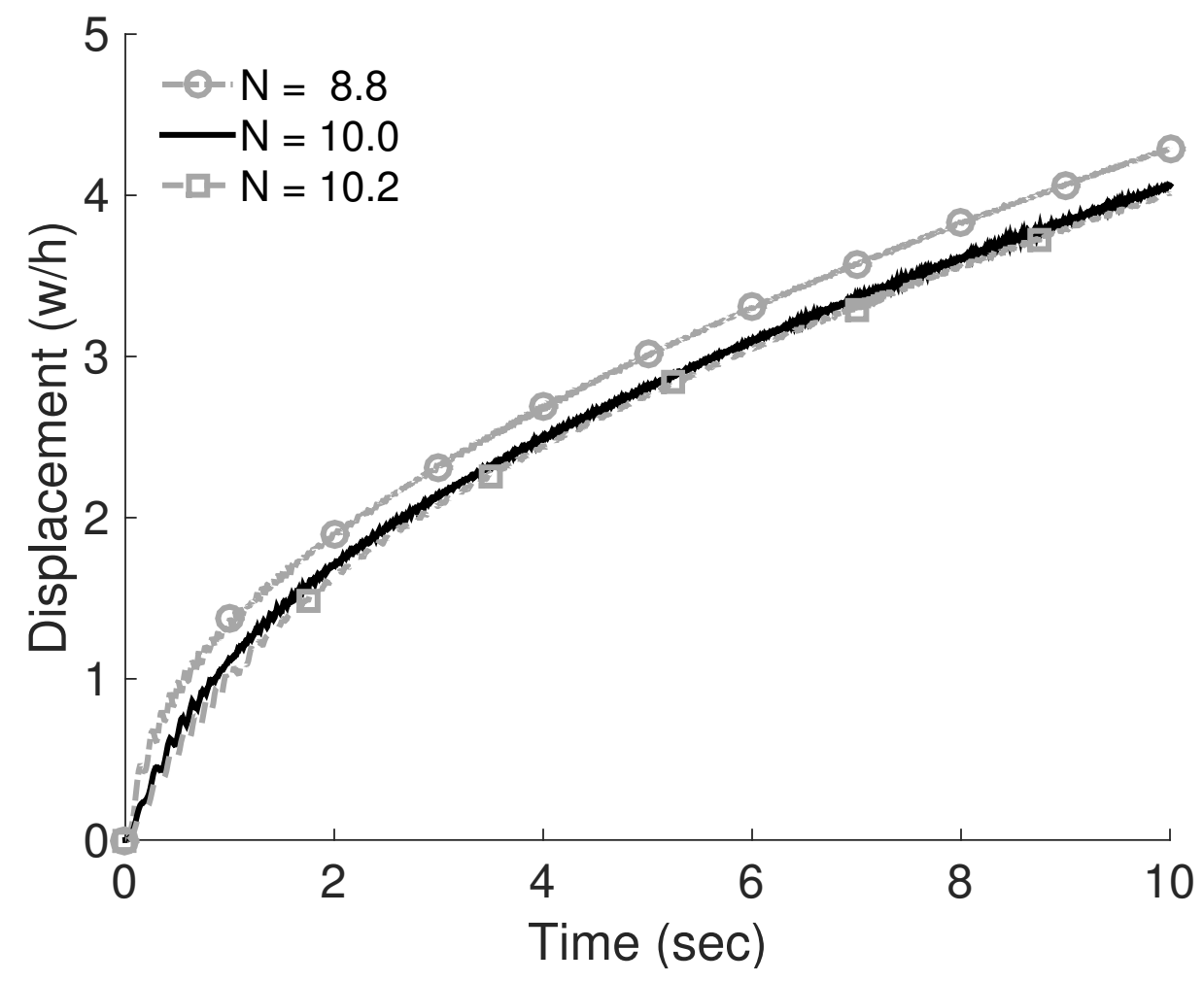

(a) Simply-supported panel.

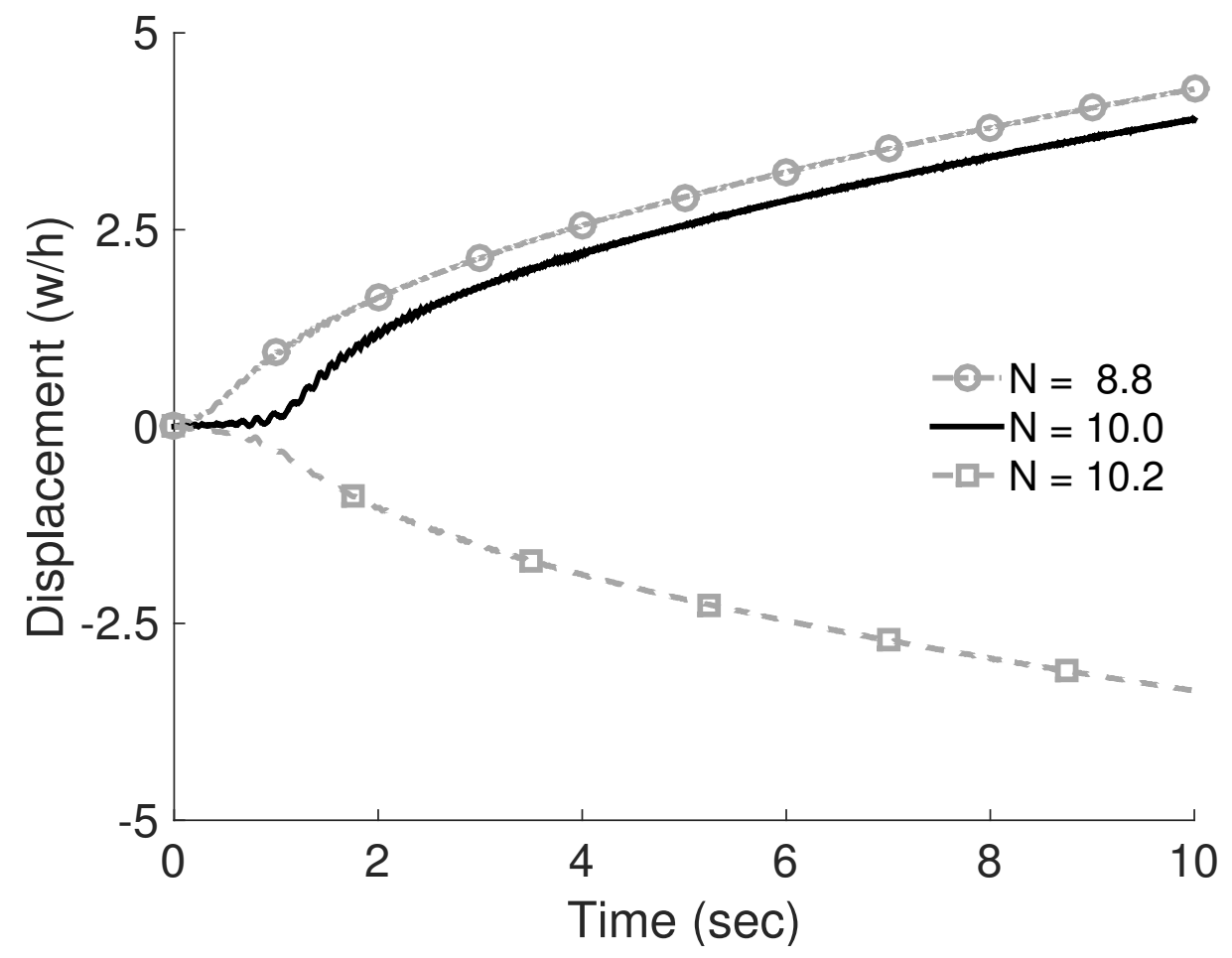

(b) Clamped panel

Figure 5.28: Center displacement with variation in transitional $N$ factor. 


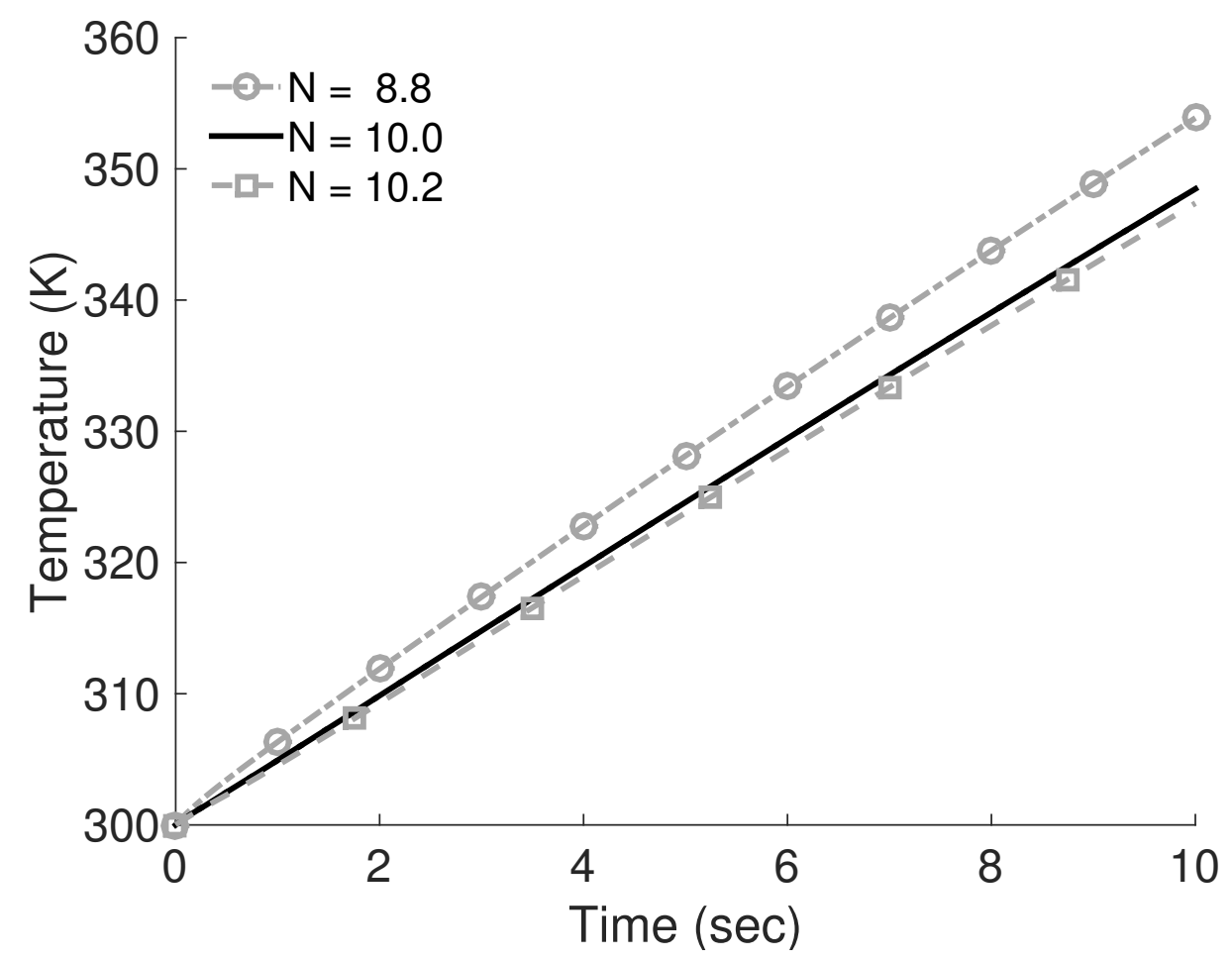

(a) Simply-supported panel.

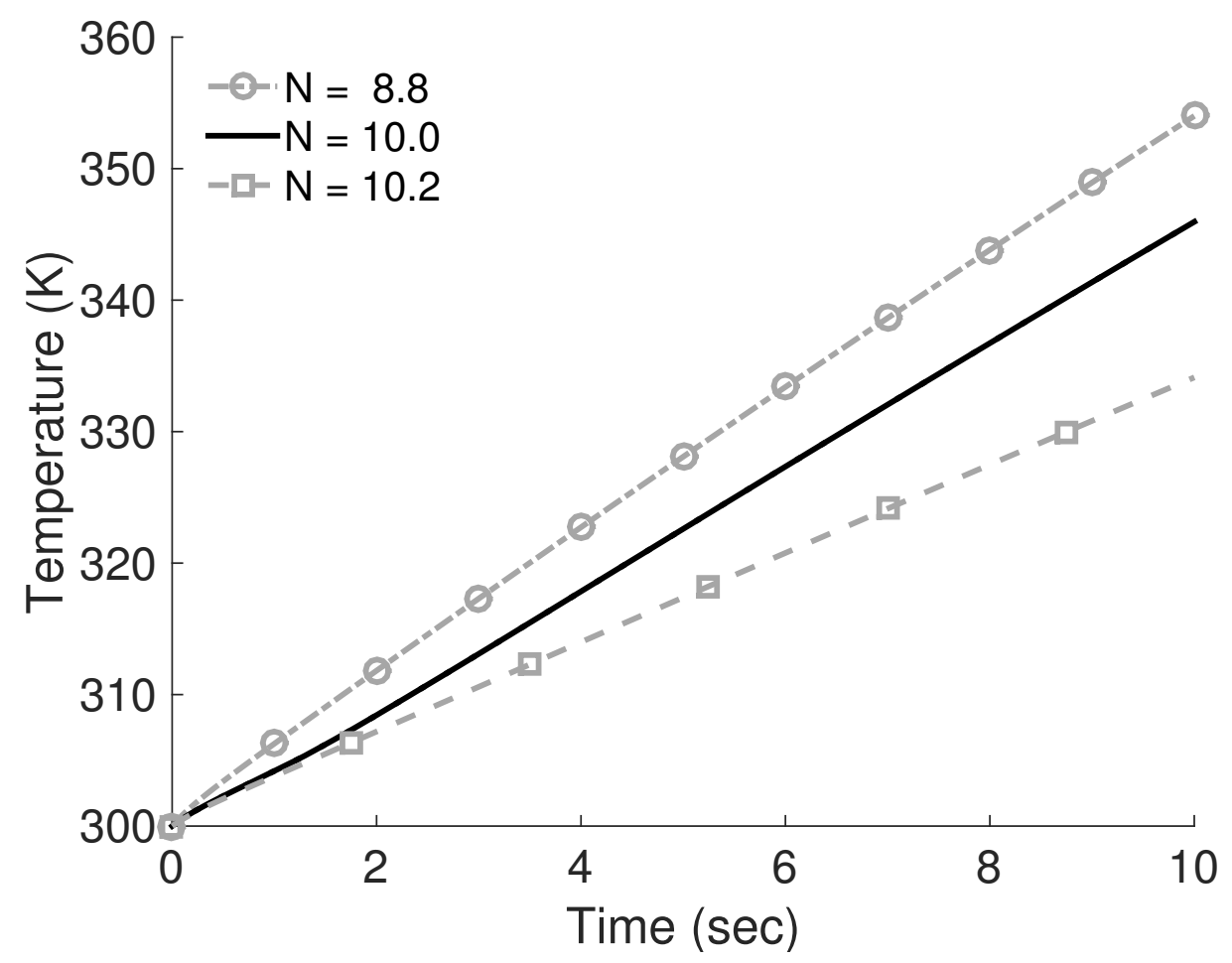

(b) Clamped panel

Figure 5.29: Average panel temperature with variation in transitional $N$ factor. 


\section{Chapter 6}

\section{Concluding Remarks}

\subsection{Principal Conclusions}

This dissertation describes research carried out to examine the interaction between boundary-layer transition and the response of aerothermally compliant structures in high-speed flows. A broad investigation into the impact of panel-scale surface deformations on hypersonic boundary-layer stability is carried out by considering a range of deformations, temperatures, and operating conditions. Models are developed for the aerothermodynamic loads resulting from a transitional boundary layer. An aerothermoelastic framework is developed that accounts for the interaction between boundary-layer transition and the thermostructural response of panels. The aerothermoelastic model is used to examine the impact of prescribed transition regions and transitional overshoot in the aerothermodynamic loads on the response of panels. Furthermore, the importance of this interaction is investigated through the comparison of panel responses obtained assuming transitional 
fluid loading, where the transition region either remains fixed or varies in time due to the panel response. The primary conclusions are:

\section{Impact of Structural Response on Boundary-layer Stability}

1. Panel deformation occurring near the leading edge of a zero pressure gradient geometry (i.e., flat plate or wedge), where the boundary layer is rapidly growing, negatively impacts stability by extending the spatial distance over which naturally occurring disturbances are unstable. Conversely, panel deformation occurring further downstream, where the boundary layer growth is small, can improve stability by reducing the spatial region of unstable amplification for fixed-frequency disturbances.

2. Convex deformations generally increase the frequency of the most unstable disturbance over the panel location whereas concave deformations decrease the frequency. This is a result of the inverse proportionality between the frequency of second-mode disturbances and boundary-layer thickness.

3. A series of 2-D panels buckled into the flow significantly disrupts the unstable growth of disturbances that are excited in their absence. The stabilizing effect results from the deformation-induced pressure gradient, which alters the boundary-layer thickness, thereby changing the frequency of the most-unstable second-mode instability. A series of panels buckled out of the flowfield results in a repeated pattern of flow expansion that thickens the boundary layer and excites low-frequency disturbances. This repeated exci- 
tation can eventually promote transition to occur upstream of that predicted for the flat wall geometry.

4. For the deformations considered in this work, boundary-layer stability is largely insensitive to the choice of simply-supported or clamped structural boundary conditions. This is demonstrated on deformations of various length with height-to-length ratios of 0.71 to 2.8 percent.

5. A constant dynamic pressure ascent, with increasing Mach number and decreasing Reynolds number, improves boundary-layer stability.

6. Higher-mode panel deformations excite a wider range of disturbance frequencies in the vicinity of the panel as compared with first-mode deformations. This is likely due to increased expansion over the aft portion of the panel, which increases boundary-layer thickness. This indicates that higher mode dominated deformations may be beneficial to flow stability in regions of low boundary-layer growth.

\section{Impact of Boundary-layer State on Structural Response}

7. Variation in the transition onset location results in larger differences in the maximum displacement, slope, and temperature as compared with variation in transition length. Altering the transition length strongly affects the chordwise thermal gradient across the panel.

8. Chordwise variation in the temperature-dependent material properties, due 
to transitional overshoot, produces non-monotonically varying aerothermoelastic stability boundaries as transition length increases.

9. The turbulent flow assumption does not provide a conservative prediction of the aerothermoelastic response of a panel. Transitional fluid loading yields significantly increased thermal gradients and can result in higher maximum temperatures. Additionally, several transitional loading configurations, with overshoot occurring near the leading edge of the panel, predict earlier flutter onset and increased strain energy accumulation during the preflutter response. Transitional overshoot occurring near the mid-chord can yield average temperatures and peak displacements exceeding that experienced by the panel subject to turbulent flow during the preflutter response.

10. Sudden transition, with overshoot in heat flux and fluctuating pressure, occurring near the leading edge of the panel provides the most conservative estimate for determining the life of the structure as it predicts the earliest flutter onset and the highest strain energy accumulation. Therefore, the design of hypersonic vehicles should account for overshoot in the aerothermodynamic loads in regions prone to transition.

\section{Interaction between Boundary-layer Transition and Structural Response}

11. For the configuration examined in this study, panel deformation has a more dominant role in altering boundary-layer stability than surface temperature. 
The effect of surface temperature on boundary-layer stability would be more pronounced for structures with higher thermal buckling temperatures.

12. For a panel positioned $2 \mathrm{~m}$ from the leading edge of a wedge body, deformation into the flowfield is found to move the predicted onset location upstream. This is a result of the deformation-induced adverse pressure gradient near the leading edge of the panel. The region of adverse pressure suppresses the growth of the boundary layer resulting in the continued excitation of high-frequency, second-mode instabilities that are unstable upstream.

13. For both the simply-supported and clamped panel, the interaction between boundary-layer transition and the structural response results in the onset location shifting upstream by more than a quarter of the panel length. The forward movement of the transition region reduces the chordwise thermal gradient and increases the peak deformation and average temperature as compared to the panel response obtained assuming a fixed transition region.

14. Accounting for the interaction between structural response and boundarylayer transition results in characteristically different panel deformations than that produced by a fixed transition region. This is mainly due to the spatial variation in the thermal bending moment resulting from the transitional heat flux.

15. The response of the clamped panel is more sensitive to the transition onset 
location than the simply-supported panel. This is shown through the application of prescribed step function thermal moments and by varying the $N$ factor corresponding to transition onset. Small variations in the transitional $N$ factor can result in the clamped panel buckling either into or out of the flowfield.

16. The movement of the transition onset location varies nonlinearly with the panel response. For either boundary condition, the majority of the transition onset movement occurs during the first $5 \mathrm{~s}$ of response. The decreased movement beyond this time is a result of the onset location being largely insensitive to panel deformations exceeding two panel thicknesses.

In summary, the aerothermoelastic response of hypersonic vehicle surface panels can alter flow stability by locally modifying the boundary-layer thickness. Variation in the boundary-layer growth can occur as a result of temperature gradients and structural deformation. Deformation was found to play the dominant role in altering the transition onset location during the aerothermoelastic response of a titanium panel with either simply-supported or clamped boundary conditions. The deformation-induced pressure gradient affects the distance over which fixedfrequency disturbances are unstable. For an otherwise zero pressure gradient geometry, the stabilizing or destabilizing effect of structural deformation depends on the distance from the boundary-layer origin. The transition onset location has a major impact on structural response as it determines the departure from lami- 
nar heat transfer rates. The rise and potential overshoot in the aerodynamic heat flux during transition alters the material properties and thermal bending moment, which can lead to structural responses that are not bounded by laminar and turbulent predictions. Movement of the transition onset location due to the changing structural state results in characteristically different panel responses.

\subsection{Key Uncertain Parameters}

Variability in the $e^{N}$ predicted transition onset location is dictated by the growth rates of instabilities (i.e., the slope of the $N$ factor curve). For smooth geometries, fixed-frequency disturbances undergo a region of unstable growth and are stabilized further downstream resulting in a monotonically increasing maximum $N$ factor envelope. The presence of localized surface perturbations (deformation and temperature) can completely damp fixed-frequency disturbances, or cause them to undergo multiple regions of unstable growth, which results in local maxima in the $N$ factor envelope. If the transitional $N$ factor is close to a local maximum the uncertainty in the predicted transition onset location greatly increases. Therefore, as compared with smooth geometries, the uncertainty associated with $e^{N}$ based transition prediction increases when accounting for aerothermally compliant structures.

The response of aerothermally compliant structures is highly dependent on the aerodynamic heat flux resulting from a transitional boundary layer. In particular, 
the transition onset location and the overshoot in heat transfer during transition have a major influence on the structural response. Both the onset location and the magnitude of the heat flux overshoot alter the material properties and thermal bending moment. In this dissertation, spatial variation in the material properties and thermal moment have been shown to alter the stability boundary and deformation of the panel. While not directly related to transitional aerothermodynamic loading, accurate characterization of the boundary conditions is essential to structural response prediction.

\subsection{Recommendations for Future Work}

In this dissertation, the effect of the aerothermoelastic behavior of structural panels on boundary-layer stability and transition is examined through static surface deformations and temperature changes. Thus, the influence of surface motion on boundary-layer stability is neglected and the conclusions drawn from this work only pertain to dynamically stable panel responses. Past experiments and computational work indicate that, in subsonic flow, perturbations induced by surface vibrations can develop into unstable Tollmien-Schlichting waves [167-170]. In Mach 6.8 flow, Fedorov and Khokhlov [44] found that hypersonic boundary layers are sensitive to vertical velocity perturbations, induced by surface vibrations, that are in resonance with the boundary-layer modes. However, in hypersonic flow, there is a large disparity between the typical frequencies associated with the second- 
mode instability and that of the dynamic response of structural components. Further study would help identify if the dynamic response of structural panels can excite either oblique first-mode or 2-D second-mode instabilities in the hypersonic flight regime.

Furthermore, this work considers how the aerothermoelastic behavior of 2-D panels can alter boundary-layer stability of a perfect gas. The consideration of 3-D structures and more realistic gas models could provide new insight into the interaction between aerothermally compliant panels and boundary layer transition.

The surrogate model for boundary-layer transition prediction, developed in this work, assumes the vehicle of interest is operating at cruise conditions (i.e., constant freestream conditions, Mach number, and angle of attack). Therefore, modifications are required to perform aerothermoelastic simulations over entire flight trajectories or mission profiles. For flight in the supersonic regime, oblique firstmode waves must be taken into consideration as they are the predominant instability below Mach 4. Also, the transition onset surrogate is generated from isothermal training data. Surface temperature gradients are accounted for by combining surrogate evaluations at the minimum and average wall temperature. Similar to surface deformations, localized temperature changes result in pressure gradients that alter stability by modifying the boundary layer thickness [82]. Accounting for wall temperature gradients in the surrogate training data would greatly improve the accuracy of the predicted transition onset location.

The transitional boundary-layer fluctuating pressure model developed in this 
work accounts for transition in a statistical sense through an intermittency function. Intermittency models are based on the growth, propagation, and interaction of turbulent spots and can directly compute transition length provided knowledge of the location of spot formation (i.e., transition onset), the spot formation rate per unit span, and a spot growth parameter. In this work, the length of the transition region is prescribed to parametrically examine its effect on panel response and because of the lack of experimental measurements of turbulent spots in hypersonic flow. However, Casper et al. [138] recently obtained the first detailed experimental measurements of the internal pressure structure of turbulent spots in a hypersonic boundary layer. Incorporating these measurements into the fluctuating pressure model would allow for the transition length to be computed from fundamental physics.

The phase angle of the fluctuating pressure load is assumed to be spatially random due to the lack of empirical correlations for transitional flows. The random representation of the spatial phase angle has been shown to underpredict the spatial coherence in a turbulent boundary layer developing over a flat plate at Mach 2.9 [17]. Furthermore, the spatial phase angle was found to have a major effect on the aerothermoelastic behavior of panels [17]. Park and Lauchle [171] simulated the transitional pressure field in subsonic flow using a model originally developed for sound radiation from exponentially growing and decaying subsonic waves. As compared with turbulent boundary layers, the transitional flow results in higher pressure levels at low streamwise wavenumbers, that can enhance fluid-structure 
coupling [171]. Future efforts should focus on leveraging DNS and experimental data of the pressure field in transitional hypersonic boundary layers to develop semi-empirical models for the spatial phase angle.

In this work, the aerothermoelastic response of a clamped panel is found to be highly sensitive to the transition onset location. This is clearly demonstrated in Fig. 5.28.b, where moving the transition onset downstream by approximately 10 $\mathrm{cm}(\Delta N=0.2)$ alters the panel buckling direction. The $N$ factor at transition varies with the turbulence intensity of the freestream [73]. Also, an experimental and computational examination of humps and dips in incompressible flow indicates that the transitional $N$ factor can vary from 7.4 to 10.0 for flow past humps and 6.7 to 9.2 for flow past dips [172]. The variability in the $N$ factor with freestream conditions and surface geometry, as well as the sensitivity of the structural response to the location of transition onset motivates the need to account for this uncertainty in the aerothermoelastic simulation of structural panels.

For the results presented in Chapter 5, relaminarization is neglected in the aerothermoelastic simulations of the interaction between boundary-layer transition and the thermostructural response of panels. However, the results in this dissertation demonstrate that surface deformation can reduce the amplification of second-mode instabilities depending on the location from the boundary-layer origin. This along with the stabilizing effect of increased wall temperature on secondmode instabilities indicate that the aerothermoelastic response of surface panels can potentially delay transition. Therefore, relaminarization of the boundary layer 
should be taken into account in future work. Previous work for incompressible flow indicates that the transitional $N$ factor for relaminarization is less than that of a fully laminar boundary layer [137]. Transition experiments are required to assess if this is also true for compressible flow.

Each of the above recommendations indicate the need for further experimental characterization of boundary-layer transition in hypersonic flow. The development and validation of improved transitional aerothermodynamic load models require experimental data that enhance our understanding of the pressure fluctuations associated with second-mode instability waves and turbulent spots in hypersonic flow. Experiments are required to identify mechanisms that result in transitional overshoot and to better quantify the magnitude above the fully turbulent level. Recent experiments in the BAM6QT have demonstrated the ability to completely damp second-mode instabilities using axisymmetric roughness strips on a flared cone [53]. Further experiments are required to measure the extent over which transition can be delayed using discrete roughness strips, and if the transitional $N$ factor changes between a smooth surface and one with isolated roughness. The largest quiet hypersonic wind tunnel in the United States is currently under construction at the University of Notre Dame [173]. Once complete, this facility will allow for the experimental examination of boundary layer transition over surface protuberances with height-to-length ratios that more closely resemble that of panel-scale deformations as opposed to roughness elements. 


\section{Bibliography}

[1] Anderson, Jr., J. D., Hypersonic and High-Temperature Gas Dynamics, American Institute of Aeronautics and Astronautics, Reston, Virginia, 2nd ed., 2006.

[2] Bertin, J. J. and Cummings, R. M., "Fifty Years of Hypersonics: Where We've Been and Where We're Going," Progress in Aerospace Sciences, Vol. 39, April 2003, pp. 511-536.

[3] Houchin II, R. F., The Rise and Fall of Dyna-Soar: A History of Air Force Hypersonic RED, 1944-1963, Ph.D. Thesis, Auburn University, Auburn, AL, 1995, pp. 1-468.

[4] Schweikart, L., "The Hypersonic Revolution, Case Studies in the History of Hypersonic Technology, vol. III: The Quest for the Orbital Jet: The National Aero-Space Plane Program (1983-1995)," Air Force History and Museum Program, 1998, Bolling AFB, DC 20332-1111.

[5] McClinton, C., "X-43 Scramjet Power Breaks the Hypersonic Barrier: Dryden Lectureship in Research for 2006," AIAA Paper 2006-1, January 2006.

[6] Rondeau, M. C. M. and Jorris, T. R., "X-51A Scramjet Demonstrator Program: WaveRider Ground and Flight Test," October 2013, SFTE 44th International/SETP Southwest Flight Test Symposium.

[7] Bowcutt, K., Paull, A., Dolvin, D., and Smart, M., "HIFiRE: An International Collaboration to Advance the Science and Technology of Hypersonic Flight," September 2012, 28th International Congress of the Aeronautical Sciences.

[8] Zuchowski, B., "Predictive Capability for Hypersonic Structural Response and Life Prediction, Phase 1 - Identification of Knowledge Gaps," Technical Report AFRL-RB-WP-TR-2010-3069, August 2010.

[9] Tzong, G., Jacobs, R., and Liguore, S., “Predictive Capability for Hypersonic Structural Response and Life Prediction: Phase 1 - Identification of Knowledge Gaps," Technical Report AFRL-RB-WP-TR-2010-3068, September 2010. 
[10] Quiroz, R., Embler, J., Jacobs, R., Tzong, G., and Liguore, S., “Predictive Capability for Hypersonic Structural Response and Life Prediction: Phase II Detailed Design of Hypersonic Cruise Vehicle Hot-Structure," Technical Report AFRL-RQ-WP-TR-2012-0265, February 2012.

[11] Zuchowski, B., "Predictive Capability for Hypersonic Structural Response and Life Prediction: Phase II - Detailed Design of Hypersonic Cruise Vehicle Hot-Structure," Technical Report AFRL-RQ-WP-TR-2012-0280, May 2012.

[12] Bertin, J. J. and Cummings, R. M., "Critical Hypersonic Aerothermodynamic Phenomena," Annual Review of Fluid Mechanics, Vol. 38, January 2006, pp. 129-157.

[13] Walker, S., Tang, M., Morris, S., and Mamplata, C., "Falcon HTV-3X - A Reusable Hypersonic Test Bed," AIAA Paper 2008-2544, May 2008.

[14] Jackson, L. R. and Dixon, S., C., "A Design Assessment of Multiwall, Metallic Stand-off, and RSI Reusable Thermal Protection Systems Including Space Shuttle Application," NASA TM 81780, April 1980.

[15] Kontinos, D. and Palmer, G., "Numerical Simulation of Metallic Thermal Protection System Panel Bowing," Journal of Spacecraft and Rockets, Vol. 36, No. 6, November-December 1999, pp. 842-849.

[16] Schlichting, H., Boundary-Layer Theory, McGraw-Hill, New York, 7th ed., 1979, pp. 1-817.

[17] Deshmukh, R., Culler, A., Miller, B., and McNamara, J., "Response of Skin Panels to Combined Self- and Boundary Layer-Induced Fluctuating Pressure," Journal of Fluids and Structures, Vol. 58, August 2015, pp. 216-235.

[18] Laganelli, A. and Wolfe, H., "Prediction of Fluctuating Pressure in Attached and Separated Turbulent Boundary-Layer Flow," Journal of Aircraft, Vol. 30, No. 6, November-December 1993, pp. 962-970.

[19] Mack, L., "Linear Stability Theory and the Problem of Supersonic BoundaryLayer Transition," AIAA Journal, Vol. 13, No. 3, 1975, pp. 278-289.

[20] Lau, K., "Hypersonic Boundary-Layer Transition: Application to HighSpeed Vehicle Design," Journal of Spacecraft and Rockets, Vol. 45, No. 2, MarchApril 2008, pp. 176-183.

[21] Wright, R. and Zoby, E., "Flight Boundary Layer Transition Measurements on a Slender Cone at Mach 20," AIAA Paper 77-719, June 1977. 
[22] Pate, S., "Dominance of Radiated Aerodynamic Noise on Boundary-Layer Transition in Supersonic-Hypersonic Wind Tunnels, Theory and Application," Technical Report AEDC-TR-77-107, March 1978.

[23] Johnson, H. and Candler, G., "Hypersonic Boundary Layer Stability Analysis Using PSE-Chem," AIAA Paper 2005-5023, June 2005.

[24] Narasimha, R., "The Laminar-Turbulent Transition Zone in the Boundary Layer," Progress in Aerospace Sciences, Vol. 22, 1985, pp. 29-80.

[25] Wadhams, T., Mundy, E., MacLean, M., and Holden, M., "Ground Test Studies of the HIFiRE-1 Transition Experiment Part 1: Experimental Results," Journal of Spacecraft and Rockets, Vol. 45, No. 6, November-December 2008, pp. 1134-1148.

[26] Franko, K. and Lele, S., "Breakdown Mechanisms and Heat Transfer Overshoot in Hypersonic Zero Pressure Gradient Boundary Layers," Journal of Fluid Mechanics, Vol. 730, 2013, pp. 491-532.

[27] Martellucci, A., Chaump, L., Rogers, D., and Smith, D., “Experimental Determination of the Aeroacoustic Environment about a Slender Cone," AIAA Journal, Vol. 11, No. 5, 1973, pp. 635-642.

[28] Casper, K., Beresh, S., Henfling, J., Spillers, R., Pruett, B., and Schneider, S., "Hypersonic Wind-Tunnel Measurements of Boundary-Layer Pressure Fluctuations," AIAA Paper 2009-4054, June 2009.

[29] Casper, K., Beresh, S., Henfling, J., Spillers, R., and Pruett, B., “High-Speed Schlieren Imaging of Disturbances in a Transitional Hypersonic Boundary Layer," AIAA Paper 2013-0376, January 2013.

[30] Lamorte, N. and Friedmann, P. P., "Hypersonic Aeroelastic and Aerothermoelastic Studies Using Computational Fluid Dynamics," AIAA Journal, Vol. 52, No. 9, September 2014, pp. 2062-2078.

[31] McNamara, J. J. and Friedmann, P. P., "Aeroelastic and Aerothermoelastic Analysis in Hypersonic Flow: Past, Present, and Future," AIAA Journal, Vol. 49, No. 6, June 2011, pp. 1089-1122.

[32] Blevins, R. D., Holehouse, I., and Wentz, K. R., "Thermoacoustic Loads and Fatigue of Hypersonic Vehicle Skin Panels," Journal of Aircraft, Vol. 30, No. 6, November-December 1993, pp. 971-978.

[33] Culler, A. J. and McNamara, J. J., "Studies on Fluid-Thermal-Structural Coupling for Aerothermoelasticity in Hypersonic Flow," AIAA Journal, Vol. 48, No. 8, August 2010, pp. 1721-1738. 
[34] Falkiewicz, N., Cesnik, C. E. S., Crowell, A. R., and McNamara, J. J., "Reduced-Order Aerothermoelastic Framework for Hypersonic Vehicle Control Simulation," AIAA Journal, Vol. 49, No. 8, August 2011, pp. 16251646.

[35] Miller, B. A., McNamara, J. J., Culler, A. J., and Spottswood, S. M., “The Impact of Flow Induced Loads on Snap-Through Behavior of Acoustically Excited, Thermally Buckled Panels," Journal of Sound and Vibration, Vol. 330, No. 23, November 2011, pp. 5736-5752.

[36] Perez, R., Wang, X., and Mignolet, M., "Nonlinear Reduced Order Models for Thermoelastodynamic Response of Isotropic and FGM Panels," AIAA Journal, Vol. 49, 2011, pp. 630-641.

[37] Ostoich, C., Bodony, D., and Geubelle, P., "Fluid-Thermal Response of Spherical Dome Under a Mach 6.59 Boundary Layer," AIAA Journal, Vol. 50, No. 12, December 2012, pp. 2791-2796.

[38] Ostoich, C., Bodony, D., and Geubelle, P., "Interaction of a Mach 2.25 Turbulent Boundary Layer with a Fluttering Panel using Direct Numerical Simulation," Physics of Fluids, Vol. 25, 2013.

[39] Weiting, A., Dechaumphai, P., Bey, K., Thornton, E., and Morgan, K., “Application of Integrated Fluid-Thermal-Structure Analysis Methods," NASA TM-100625, 1988.

[40] Glass, C. and Hunt, L., "Aerothermal Tests of Quilted Dome Models on a Flat Plate at a Mach Number of 6.5," NASA TP-2804, 1988.

[41] Gordon, R. W. and Hollkamp, J. J., "Coupled Structural-Acoustic Response Prediction with Complex Modal Methods," AIAA Paper 2009-2307, May 2009.

[42] Mack, L. M., Stability of the Compressible Laminar Boundary Layer According to a Direct Numerical Solution, May 1965, pp. 329-362, AGARD-CP-97.

[43] Schneider, S., "Effects of Roughness on Hypersonic Boundary Layer Transition," Journal of Spacecraft and Rockets, Vol. 45, No. 2, March-April 2008, pp. 193-209.

[44] Fedorov, A. and Khokhlov, A., "Receptivity of Hypersonic Boundary Layer to Wall Disturbances," Theoretical and Computational Fluid Dynamics, Vol. 15, September 2002, pp. 231-254.

[45] Wang, X. and Zhong, X., "Receptivity of a Hypersonic Flat-Plate Boundary Layer to Three-Dimensional Surface Roughness," Journal of Spacecraft and Rockets, Vol. 45, No. 6, November-December 2008, pp. 1165-1175. 
[46] Berry, S. and Horvath, T., "Discrete-Roughness Transition for Hypersonic Flight Vehicles," Journal of Spacecraft and Rockets, Vol. 45, No. 2, March-April 2008, pp. 216-227.

[47] Wang, X. and Zhong, X., "Effect of Wall Perturbations on the Receptivity of a Hypersonic Boundary Layer," Physics of Fluids, Vol. 21, No. 4, 2009, pp. 044101-19.

[48] Fujii, K., "Experiment of the Two-Dimensional Roughness Effect on Hypersonic Boundary-Layer Transition," Journal of Spacecraft and Rockets, Vol. 43, No. 4, July-August 2006, pp. 731-738.

[49] Marxen, O., Iaccarino, G., and Shaqfeh, E., “Disturbance Evolution in a Mach 4.8 Boundary Layer with Two-Dimensional Roughness-Induced Separation and Shock," Journal of Fluid Mechanics, Vol. 648, 2010, pp. 435-469.

[50] Bountin, D., Chimitov, T., and Maslov, A., "Stabilization of a Hypersonic Boundary Layer Using A Wavy Surface," AIAA Journal, Vol. 51, No. 5, May 2013, pp. 1203-1210.

[51] Duan, L., Wang, X., and Zhong, X., "Stabilization of a Mach 5.92 Boundary Layer by Two-Dimensional Finite-Height Roughness," AIAA Journal, Vol. 51, No. 1, November 2013, pp. 266-270.

[52] Fong, K., Wang, X., and Zhong, X., "Numerical Simulation of Roughness Effect on the Stability of a Hypersonic Bouondary Layer," Computers and Fluids, Vol. 96, 2014, pp. 350-367.

[53] Fong, K., Wang, X., Huang, Y., Zhong, X., McKiernan, G., Fisher, R., and Schneider, S., "Second Mode Suppression in Hypersonic Boundary Layer by Roughness: Design and Experiments," AIAA Journal, Vol. 53, No. 10, 2015, pp. 3138-3144.

[54] Reynolds, O., "An Experimental Investigation of the Circumstances Which Determine Whether the Motion of Water Shall Be Direct or Sinuous, and of the Law of Resistance in Parallel Channels," Philosophical Transactions of the Royal Society of London, Vol. 174, March 1883, pp. 935-982.

[55] Rott, N., "Note on the History of the Reynolds Number," Annual Review of Fluid Mechanics, Vol. 22, 1990, pp.1-11.

[56] Gersten, K., Hermann Schlicting and the Boundary-Layer Theory. In Hermann Schlicting-100 Years, Springer-Verlag, Berlin Heidelberg, 2009, pp. 3-17.

[57] Shubauer, G. B. and Skramstad, H. K., "Laminar-Boundary-Layer Oscillations and Transition on a Flat Plate," NACA Report 909, 1948. 
[58] White, F. M., Viscous Fluid Flow, McGraw-Hill, New York, 2nd ed., 1991, pp. $1-614$.

[59] Reshotko, E., "Boundary-Layer Stability and Transition," Annual Review of Fluid Mechanics, Vol. 8, 1976, pp. 311-349.

[60] Juniper, M., Hanifi, A., and Theofilis, V., "Modal Stability Theory," Applied Mechanics Review, Vol. 66, 2014, pp. 1-22.

[61] Lees, L. and Lin, C., "Investigation of the Stability of the Laminar Boundary Layer in a Compressible Fluid," NACA TN 1115, September 1946.

[62] Mack, L. M., “Boundary Layer Stability Theory," Jet Propulsion Lab, Rept. 900-277 Rev. A, November 1965, Pasadena, CA.

[63] Mack, L. M., "On the Inviscid Acoustic-Mode Instability of Supersonic Shear Flows, Part 1: Two-Dimensional Waves," Theoretical and Computational Fluid Dynamics, Vol. 2, July 1990, pp. 97-123.

[64] Cebeci, T. and Cousteix, J., Modeling and Computation of Boundary-Layer Flows, Horizons Publishing, Long Beach, California, 2nd ed., 2005, pp. 456-462.

[65] Bertolotti, F. and Herbert, T., "Analysis of the Linear Stability of Compressible Boundary Layers Using the PSE," Theoretical and Computational Fluid Dynamics, Vol. 3, April 1991, pp. 117-124.

[66] Chang, C. L., Malik, M. R., Erlebacher, G., and Hussaini, M. Y., "Linear and Nonlinear PSE for Compressible Boundary Layers," NASA Contractor Report 191537, December 1993, ICASE Report No. 93-70.

[67] Masad, J. A. and Abid, R., "On Transition in Supersonic and Hypersonic Boundary Layers," International Journal of Engineering Sciences, Vol. 33, No. 13, 1995, pp. 1893-1919.

[68] Theofilis, V., "Advances in Global Linear Instability Analysis of Nonparallel and Three-Dimensional Flows," Progress in Aerospace Sciences, Vol. 39, 2003, pp. 249-315.

[69] Stetson, K. F., "On Predicting Hypersonic Boundary Layer Transition," AFWAL-TM-87-160-FIMG, March 1987.

[70] Smith, A. and Gamberoni, N., "Transition, Pressure Gradient, and Stability Theory," Douglas Aircraft Co. Rep. ES26388, 1956, El Segundo, CA.

[71] Van Ingen, J. L., "A Suggested Semi-Empirical Method for the Calculation of the Boundary Layer Transition Region," Report V.T.H. 74, 1956, Dept. of Aeronautics and Engineering, Institute of Technology, Delft, Holland. 
[72] Jaffe, N., Okamura, T., and Smith, A., "Determination of Spatial Amplification Factors and Their Application to Predicting Transition," AIAA Journal, Vol. 8, No. 2, February 1970, pp. 301-308.

[73] Mack, L. M., Transition Prediction and Linear Stability Theory, 1977, pp. 1-22, AGARD-CP-224.

[74] Beckwith, I., "Development of a High Reynolds Number Quiet Tunnel for Transition Research," AIAA Journal, Vol. 13, No. 3, March 1975, pp. 300-306.

[75] Stetson, K. F., “Comments on Hypersonic Boundary-Layer Transition," Technical Report WRDC-TR-90-3057, September 1990.

[76] Kimmel, R. L., "The Effect of Pressure Gradients on Transition Zone Length in Hypersonic Boundary Layers," Transactions of the ASME, Vol. 119, March 1997, pp. 36-41.

[77] Lees, L., "The Stability of the Laminar Boundary Layer in a Compressible Fluid," NACA Rep. 876, 19447.

[78] Demetriades, A., "New Experiments on Hypersonic Boundary Layer Stability Including Wall Temperature Effects," Proceedings of the 1978 Heat Transfer and Fluid Mechanics Institute, Pullman, Washington, June 1978, p. p. 39, Stanford University Press.

[79] Stetson, K. and Kimmel, R., "On Hypersonic Boundary-Layer Stability," AIAA Paper 92-0737, January 1992.

[80] Dovgal, A., Levchenko, V., and Timofeev, V., Laminar-Turbulent Transition, Springer-Verlag, Berlin, 1990, pp. 113-121, edited by Arnal, D. and Michel, R.

[81] Masad, J. A. and Nayfeh, A., "Laminar Flow Control of Subsonic Boundary Layers by Suction and Heat-Transfer Strips," Physics of Fluids, Vol. 4, No. 6, June 1992, pp. 1259-1272.

[82] Fedorov, A., Soudakov, V., Egorov, I., Sidorenko, A., Gromyko, Y., Bountin, D., Polivanov, P., and Maslov, A., "High-Speed Boundary-Layer Stability on a Cone with Localized Wall Heating or Cooling," AIAA Journal, Vol. 53, No. 9, September 2015, pp. 2512-2524.

[83] Soudakov, V., Egorov, I., and Fedorov, A., "Numerical Simulation of Receptivity of a Hypersonic Boundary Layer over a Surface with Temperature Jump," Proceedings of the 6th European Symposium on Aerothermodynamics for Space Vehicles, Versailles, France, 2008, ESA SP-659. 
[84] Dryden, H. L., "Review of Published Data on the Effect of Roughness on Transition from Laminar to Turbulent Flow," Journal of the Aeronautical Sciences, Vol. 20, July 1953, pp. 477-482.

[85] Reda, D., "Review and Synthesis of Roughness-Dominated Transition Correlations for Reentry Applications," Journal of Spacecraft and Rockets, Vol. 39, No. 2, March-April 2002, pp. 161-167.

[86] Fage, A., "The Smallest Size of a Spanwise Surface Corrugation which Affects Boundary-layer Transition on an Aerofoil," British Ministry of Supply, Aeronautical Research Council Reports and Memoranda, R\&M No. 2120, January 1943.

[87] Carmichael, B., "Surface Waviness Criteria for Swept and Unswept Laminar Suction Wings," Northrop Aircraft Report No. NOR-59-438 (BLC123), 1957.

[88] James, C. S., "Boundary-Layer Transition on Hollow Cylinders in Supersonic Free Flight as Affected by Mach Number and a Screwthread Type of Surface Roughness," NASA TR-Memo 1-20-59A, February 1959.

[89] Holloway, P. F. and Sterrett, J. R., "Effect of Controlled Surface Roughness on Boundary-Layer Transition and Heat Transfer at Mach Numbers of 4.8 and 6.0," NASA TN D-2054, April 1964.

[90] Saric, W., Carrillo, R., and Reibert, M., "Leading-Edge Roughness as a Transition Control Mechanisim," AIAA Paper 1998-98, 1998.

[91] Saric, W. and Reed, H., "Supersonic Laminar Flow Control on Swept Wings Using Distributed Roughness," AIAA Paper 2002-0147, January 2002.

[92] Tang, Q., Zhu, Y., Chen, X., and Lee, C., “Development of Second-Mode Instability in a Mach 6 Flat Plate Boundary Layer with Two-Dimensional Roughness," Physics of Fluids, Vol. 27, 2015.

[93] Glass, C. E. and Hunt, L. R., "Aerothermal Tests of Spherical Dome Protuberances on a Flat Plate at a Mach Number of 6.5," NASA TP-2631, 1986.

[94] Berry, S., Horvath, T., Hollis, B., Thompson, R., and Hamilton II, H., "X33 Hypersonic Boundary-Layer Transition," Journal of Spacecraft and Rockets, Vol. 38, No. 5, September-October 2001, pp. 646-656.

[95] Johnson, H., Alba, C., Bartkowicz, M., Drayna, T., and Candler, G., “Design Optimization of Hypersonic Vehicles for Boundary-Layer Stability," AIAA Paper 2008-6221, August 2008. 
[96] Dugundji, J. and Calligeros, J. M., "Similarity Laws for Aerothermoelastic Testing," Journal of the AeroSpace Sciences, Vol. 29, No. 8, August 1962, pp. 935-950.

[97] Dixon, S., Griffith, G., and Bohon, H., "Experimental Investigation at Mach Number 3.0 of the Effects of Thermal Stress and Buckling on the Flutter of Four-Bay Aluminum Alloy Panels with Length-Width ratios of 10," NASA TN D-921, 1961.

[98] Guy, L. and Bohon, H., "Flutter of Aerodynamically Heated Aluminum Alloy and Stainless-Steel Panel with Length-Width Ratio of 10 at Mach Number of 3.0," NASA TN D-1353, 1962.

[99] Dowell, E. H. and Voss, H. M., "Theoretical and Experimental Panel Flutter Studies in the Mach Number Range of 1.0 to 5.0," AIAA Journal, Vol. 3, No. 12, December 1965, pp. 2292-2304.

[100] Spottswood, S., Eason, T., and Beberniss, T., "Influence of Shock-Boundary Layer Interactions on the Dynamic Response of a Flexible Panel," Proceedings, International Conference on Noise and Vibration Engineering (ISMA), Leuven, Belgium, September 2012.

[101] Culler, A. J. and McNamara, J. J., "Impact of Fluid-Thermal-Structural Coupling on Response Prediction of Hypersonic Skin Panels," AIAA Journal, Vol. 49, No. 11, November 2011, pp. 2393-2406.

[102] LaFontaine, J., Gogulapati, A., and McNamara, J., "Effects of Strain Hardening on Response of Sking Panels in Hypersonic Flow," AIAA Journal, Vol. 54, No. 6, June 2016, pp. 1974-1986.

[103] McNamara, J. J., Crowell, A. R., Friedmann, P. P., Glaz, B., and Gogulapti, A., "Approximate Modeling of Unsteady Aerodynamics for Hypersonic Aeroelasticity," Journal of Aircraft, Vol. 47, No. 6, November-December 2010, pp. 1932-1945.

[104] Crowell, A. R., McNamara, J. J., and Miller, B. A., "Hypersonic Aerothermoelastic Response Prediction of Skin Panels Using Computational Fluid Dynamic Surrogates," Journal of Aeroelasticity and Structural Dynamics, Vol. 2, No. 2, June 2011, pp. 3-30.

[105] Crowell, A. R., Miller, B. A., and McNamara, J. J., "Model Reduction of Computational Aerothermodynamis for Hypersonic Aerothermoelasticity," AIAA Journal, Vol. 50, No. 1, January 2012, pp. 74-84.

[106] Brouwer, K. R., Crowell, A. R., and McNamara, J. J., "Rapid Prediction of Unsteady Aeroelastic Loads in Shock-Dominated Flows," AIAA Paper 20150687, January 2015. 
[107] Zettl, D., Dreyer, E. R., Grier, B. J., and McNamara, J. J., “Rapid Steady-State Pressure Prediction for Ultra High-Speed Vehicles," AIAA Paper 2016-1323, January 2016.

[108] Crowell, A. R., Miller, B. A., and McNamara, J. J., "Robust and Efficient Treatment of Temperature Feedback in Fluid-Thermal-Structural Analysis," AIAA Journal, Vol. 52, No. 11, November 2014, pp. 2395-2413.

[109] Falkiewicz, N. and Cesnik, C. E. S., "Proper Orthogonal Decomposition for Reduced-Order Thermal Solution in Hypersonic Aerothermoelastic Simulations," AIAA Journal, Vol. 49, No. 5, May 2011, pp. 994-1009.

[110] Mignolet, M. P., Przekop, A., Rizzi, S. A., and Spottswood, S. M., “A Review of Indirect/Non-intrusive Reduced Order Modeling of Nonlinear Geometric Structures," Journal of Sound and Vibration, Vol. 332, October 2013, pp. 24372460.

[111] Schultz-Grunow, F., "New Frictional Resistance Law for Smooth Plates," NACA TM 986, September 1941.

[112] Owens, F. K. and Horstman, C. C., "Hypersonic Transitional Boundary Layers," AIAA Journal, Vol. 10, No. 6, June 1972, pp. 769-775.

[113] Chynoweth, B. C., Ward, C. A. C., Greenwood, R. T., McKiernan, G. R., Fisher, R. A., and Schneider, S. P., "Measuring Transition and Instabilities in a Mach 6 Hypersonic Quiet Wind Tunnel," AIAA Paper 2014-2643, June 2014.

[114] Sivasubramanian, J. and Fasel, H. F., "Nonlinear Stages of Transition and Breakdown in a Boundary Layer on a Sharp Cone at Mach 6," AIAA Paper 2012-0087, January 2012.

[115] Hader, C. and Fasel, H. F., "Laminar-Turbulent Transition on a Flared Cone at Mach 6," AIAA Paper 2016-3344, June 2016.

[116] Reshotko, E., “Transition Issues at Hypersonic Speeds," AIAA Paper 2006707, January 2006.

[117] Wright, M. J., Candler, G. V., and Bose, D., “Data-Parallel Line Relaxation Method for the Navier-Stokes Equations," AIAA Journal, Vol. 36, No. 9, September 1998, pp. 1603-1609.

[118] Johnson, H., "STABL: Stability and Transition Analysis for Hypersonic Boundary Layers Program Reference," University of Minnesota, Minneapolis, MN, June 2010. 
[119] Johnson, H. B. and Candler, G. V., "Analysis of Laminar-Turbulent Transition in Hypersonic Flight Using PSE-Chem," AIAA Paper 2006-3057, June 2006.

[120] Robarge, T. W. and Schneider, S. P., "Laminar Boundary-Layer Instabilities on Hypersonic Cones: Computations for Benchmark Experiments," AIAA Paper 2005-5024, June 2005.

[121] MacLean, M., Wadhams, T., Holden, M., and Johnson, H., "Ground Test Studies of the HIFiRE-1 Transition Experiment Part 2: Computational Analysis," Journal of Spacecraft and Rockets, Vol. 45, No. 6, November-December 2008, pp. 1149-1164.

[122] Johnson, H., Thermochemical Interactions in Hypersonic Boundary Layer Stability, Ph.D.Thesis, University of Minnesota, Minneapolis, MN, 2000, pp. 1-128.

[123] Hornung, H. G., "Hypersonic Real-Gas Effects on Transition," IUTAM Symposium on One Hundred Years of Boundary Layer Research, Gottingen, Germany, August 2006, pp. 335-344.

[124] Herbert, T., "Boundary-Layer Transition: Analysis and Prediction Revisited," AIAA Paper 91-0737, January 1991.

[125] Herbert, T., "Parabolized Stability Equations," Annual Review of Fluid Mechanics, Vol. 29, 1997, pp. 245-283.

[126] Forrester, A., Sobester, A., and Keane, A., Engineering Design via Surrogate Modelling: A Practical Guide, John Wiley \& Sons, New York, NY, 2008.

[127] Simpson, T. W., Mauery, T. M., Korte, J. J., and Mistree, F., “Kriging Models for Global Approximation in Simulation-Based Multidisciplinary Design Optimization," AIAA Journal, Vol. 39, No. 12, December 2001, pp. 2233-2241.

[128] Krige, D. G., "A Statistical Approach to Some Basic Mine Valuation Problems on the Witwatersrand," Journal of the Chemical, Metallurgical and Mining Engineering Society of South Africa, Vol. 52, No. 6, December 1951, pp. 119-139.

[129] Matheron, G., "Principles of Geostatistics," Economic Geology, Vol. 58, 1963, pp. 1246-1266.

[130] Sacks, J., Welch, W. J., Mitchell, T. J., and Wynn, H. P., "Design and Analysis of Computer Experiments," Statistical Science, Vol. 4, No. 4, 1989, pp. 409435.

[131] Lophaven, S. N., Nielsen, H. B., and Sondergaard, J., “DACE A Matlab Kriging Toolbox Version 2.0," Technical Report IMM-TR-2002-12, August 2002. 
[132] Lighthill, M., "Oscillating Airfoils at High Mach Numbers," Journal of the Aeronautical Sciences, Vol. 20, No. 6, June 1953, pp. 402-406.

[133] Ashley, H. and Zartarian, G., "Piston Theory - A New Aerodynamic Tool for the Aeroelastician," Journal of the Aeronautical Sciences, Vol. 23, No. 12, December 1956, pp. 1109-1118.

[134] Eckert, E. R. G., “Engineering Relations for Heat Transfer and Friction in High-Velocity Laminar and Turbulent Boundary-Layer Flow Over Surfaces With Constant Pressure and Temperature," Transactions of the ASME, Vol. 78, No. 6, August 1956, pp. 1273-1283.

[135] Miller, B. A., Loosely Coupled Time Integration of Fluid-Thermal-Structural Interactions in Hypersonic Flows, Ph.D. Thesis, The Ohio State University, Columbus, OH, 2015, pp. 1-198.

[136] Miller, B. A. and McNamara, J. J., “Time-Marching Considerations for Response Prediction of Structures in Hypersonic Flows," AIAA Journal, Vol. 53, No. 10, October 2015, pp. 3028-3038.

[137] Crouch, J. D., Crouch, I. W. M., and Ng, L. L., “Transition Prediction for Three-Dimensional Boundary Layers in Computational Fluid Dynamics Applications," AIAA Journal, Vol. 40, No. 8, August 2002, pp. 1536-1541.

[138] Casper, K., Beresh, S., and Schneider, S., "Pressure Fluctuations Beneath Instability Wavepackets and Turbulent Spots in a Hypersonic Boundary Layer," Journal of Fluid Mechanics, Vol. 756, 2014, pp. 1058-1091.

[139] Laganelli, A. and Howe, J., "Prediction of Pressure Fluctuations Associated with Maneuvering Re-entry Weapons," AFFDL-TR-77-59, July 1977.

[140] Blevins, R. D., Bofilios, D., Holehouse, I., Hwa, V. W., Tratt, M. D., Laganelli, A. L., Pozefsky, P., and Pierucci, M., "Thermo-Vibro-Acoustic Loads and Fatigue of Hypersonic Flight Vehicle Structure - Phase II Report," Rohr Industries, Inc., RHR 89-202, November 1989.

[141] Bull, M. K., “Wall-Pressure Fluctuations Beneath Turbulent Boundary Layers: Some Reflections on Forty Years of Research," Journal of Sound and Vibration, Vol. 190, No. 3, November 1996, pp. 299-315.

[142] Beresh, S. J., Henfling, J. F., Spillers, R. W., and Pruett, B. O., "Fluctuating Wall Pressures Measured Beneath a Supersonic Turbulent Boundary Layer," Physics of Fluids, Vol. 23, No. 075110, 2011, pp. 1-16.

[143] Dhawan, S. and Narasimha, R., "Some Properties of Boundary Layer Flow During the Transition from Laminar to Turbulent Motion," Journal of Fluid Mechanics, Vol. 3, April 1958, pp. 418-436. 
[144] Emmons, H., "The Laminar-Turbulent Transition in a Boundary Layer-Part I," Journal of the Aeronautical Sciences (Institute of the Aeronautical Sciences), Vol. 18, 1951, pp. 490-498.

[145] Emmons, H. and Bryson, A., "The Laminar-Turbulent Transition in a Boundary Layer-Part II," Proceedings of the First U.S. National Congress of Theoretical and Applied Mechanices, 1952, pp. 859-868.

[146] Chaump, L. E., Martellucci, A., and MonFort, A., "Aeroacoustic Loads Associated with High Beta Re-Entry Vehicles," Technical Report AFFDL-TR-72138, Volume 1, May 1973.

[147] Casper, K., Hypersonic Wind-Tunnel Measurements of Boundary-Layer Pressure Fluctuations, M.S. Thesis, Purdue University, West Lafayette, IN, 2009, pp. $1-123$.

[148] Miller, B., Crowell, A., and McNamara, J., "Modeling and Analysis of Shock Impingements on Thermo-Mechanically Compliant Surface Panels," AIAA Paper 2012-1548, April 2012.

[149] Deveikis, W. and Hunt, L., "Loading and Heating of a Large Flat Plate at Mach 7 in the Langley 8-Foot High-Temperature Structures Tunnel," NASA TN D-7275, September 1973.

[150] Sherman, M. and Nakamura, T., "Flight Test Measurements of Boundary Layer Transition on a Nonablating 22-Deg. Cone," Journal of Spacecraft and Rockets, Vol. 7, No. 2, February 1970, pp. 137-142.

[151] Stetson, K., Thompson, E., Donaldson, J., and Siler, L., “Laminar Boundary Layer Stability Experiments on a Cone at Mach 8, Part 2: Blunt Cone," AIAA Paper 84-0006, January 1984.

[152] Malik, M. R., Spall, R. E., and Chang, C. L., "Effect of Nose Bluntness on Boundary Layer Stability and Transition," AIAA Paper 90-0112, January 1990.

[153] Malik, M. R., "Hypersonic Flight Transition Data Analysis Using Parabolized Stability Equations with Chemistry Effects," Journal of Spacecraft and Rockets, Vol. 40, No. 3, May-June 2003.

[154] Zhong, X., "Numerical Simulation and Experimental Comparison of Hypersonic Boundary Layer Instability over a Blunt Cone," AIAA Paper 2004-2244, June 2004.

[155] Theofilis, V., Hein, S., and Dallmann, U., "On the Origins of Unsteadiness and Three-Dimensionality in a Laminar Separation Bubble," Philosophical Transactions of the Royal Society, Vol. 358, 2000, pp. 3229-3246. 
[156] Fong, K., Wang, X., and Zhong, X., "Stabilization of Hypersonic Boundary Layer by 2-D Surface Roughness," AIAA Paper 2013-2985, June 2013.

[157] Marxen, O. and Iaccarino, G., "Numerical Simulation of the Effect of a Roughness Element on High-Speed Boundary-Layer Instability," AIAA Paper 2008-4400, June 2008.

[158] Fong, K., Wang, X., and Zhong, X., "Finite Roughness Effect on Modal Growth of a Hypersonic Boundary Layer," AIAA Paper 2012-1085, January 2012.

[159] Holden, M. S., "An Experimental Investigation of Turbulent Boundary Layers at High Mach Number," NASA Contractor Report 112147, 1972.

[160] Juliano, T., Kimmel, R., Willems, S., Gulhan, A., and Schneider, S., “HIFiRE1 Surface Pressure Fluctuations from High Reynolds, High Angle Ground Test," AIAA Paper 2014-0429, January 2014.

[161] Wertz, J., Letcher, T., Shen, M.-H, H., Scott-Emuakpor, O., George, T., and Cross, C., "An Energy-Based Axial Isothermal-Mechanical Fatigue Lifing Method," Journal of Engineering for Gas Turbines and Power, Vol. 134, No. 10, October 2012.

[162] Scott-Emuakpor, O., George, T., Cross, C., and Shen, M.-H, H., "Multi-Axial Fatigue-Life Prediction via a Strain-Energy Method," AIAA Journal, Vol. 48, No. 1, January 2010, pp. 63-72.

[163] Myers, D. E., Martin, C. J., and Blosser, M. L., "Parametric Weight Comparison of Advanced Metallic, Ceramic Tile, and Ceramic Blanket Thermal Protection Systems," NASA TM 2000-210289, June 2000.

[164] Juliano, T., Kimmel, R., Willems, S., Gulhan, A., and Wagnild, R., "HIFiRE1 Boundary-Layer Transition: Ground Test Results and Stability Analysis," AIAA Paper 2015-1736, January 2015.

[165] Marineau, E. C., "Prediction Methodology for 2nd Mode Dominated Boundary Layer Transition in Hypersonic Wind Tunnels," AIAA Paper 2016-0597, January 2016.

[166] Crouch, J. D. and Ng, L. L., "Variable N-Factor Method for Transition Prediction in Three-Dimensional Boundary Layers," AIAA Journal, Vol. 38, No. 2, February 2000, pp. 211-216.

[167] Tumin, A. and Fedorov, A., "Instability Wave Excitation by a Localized Vibrator in the Boundary Layer," Journal of Applied Mechanics and Technical Physics, Vol. 25, No. 6, November 1984, pp. 867-873. 
[168] Maestrello, L. and Grosveld, "Transition Control of Instability Waves over an Acoustically Excited Flexible Surface," AIAA Journal, Vol. 30, No. 3, March 1992, pp. 665-670.

[169] Visbal, M. and Gordnier, R., "Numerical Simulation of the Interaction of a Transitional Boundary Layer with a 2-D Flexible Panel in the Subsonic Regime," Journal of Fluids and Structures, Vol. 19, May 2004, pp. 881-903.

[170] Wang, Z., Yeo, K., and Khoo, B., "Spatial Direct Numerical Simulation of Transitional Boundary Layer over Compliant Surfaces," Computers and Fluids, Vol. 34, August 2005, pp. 1062-1095.

[171] Park, S. and Lauchle, G. C., "Wall Pressure Fluctuation Spectra due to Boundary-Layer Transition," Journal of Sound and Vibration, Vol. 319, June 2009, pp. 1067-1082.

[172] Nayfeh, A., Ragab, S., and Al-Maaitah, A., "Effect of Bulges on the Stability of Boundary Layers," Physics of Fluids, Vol. 31, No. 4, April 1988, pp. 796-806.

[173] Welding, N., "Hypersonics on the QT at Notre Dame," https:/ / engineering.nd.edu/highlights/hypersonics-on-the-qt-at-notredame, August 2015. 